Illinois State University

ISU ReD: Research and eData

Theses and Dissertations

$5-19-2015$

\title{
Effects Of Explicit Reading Comprehension Strategy Instruction For English Learners With Specific Learning Disabilities
}

Sara Lynn Jozwik

Illinois State University, jozwik@uwm.edu

Follow this and additional works at: https://ir.library.illinoisstate.edu/etd

Part of the Bilingual, Multilingual, and Multicultural Education Commons, Other Education Commons, Reading and Language Commons, Special Education Administration Commons, and the Special Education and Teaching Commons

\section{Recommended Citation}

Jozwik, Sara Lynn, "Effects Of Explicit Reading Comprehension Strategy Instruction For English Learners With Specific Learning Disabilities" (2015). Theses and Dissertations. 415.

https://ir.library.illinoisstate.edu/etd/415

This Dissertation is brought to you for free and open access by ISU ReD: Research and eData. It has been accepted for inclusion in Theses and Dissertations by an authorized administrator of ISU ReD: Research and eData. For more information, please contact ISUReD@ilstu.edu. 


\title{
EFFECTS OF EXPLICIT READING COMPREHENSION STRATEGY INSTRUCTION FOR ENGLISH LEARNERS WITH SPECIFIC LEARNING DISABILITIES
}

\author{
Sara L. Jozwik
}

244 Pages

August 2015

In this intervention study, I engaged principles of culturally responsive research to examine the effectiveness of explicit reading comprehension strategy instruction for English Learners (ELs) with specific learning disabilities (SLD). This study replicated and extended previous research (Jitendra, Hoppes, \& Xin, 2000) by modifying instruction found to be effective for native English speakers (i.e., explicit reading comprehension strategy instruction with a self-monitoring procedure). Modifications included: (a) integrating culturally relevant text, (b) providing native language support, and (c) melding strategies from the fields of teaching English as a second language and special education. Through a co-teaching model, I provided instruction to four participants during a 135-min literacy block in a fifth-grade general education classroom for 13 weeks. A multiple probe across participants design (Gast \& Ledford, 2010) evaluated effects of instruction on two dependent variables: (a) participants' sophistication with applying comprehension thinking strategies while reading, as measured by comprehension thinking strategy rubrics (Keene, 2006) and (b) participants' 
comprehension, as measured through percentage accuracy with responding to openended, researcher-developed literal and inferential comprehension questions. I assessed maintenance of effects for 2 to 8 weeks after participants exited the intervention condition. I assessed generalization to on-grade-level text and to a standardized achievement test (Woodcock Johnson Tests of Academic Achievement III-R; Woodcock, McGrew, \& Mather, 2001). Additionally, I examined participants' self-efficacy as readers at pre- and posttest by collecting information from the Motivation to Read Profile survey and interview (Gambrell, Palmer, Coddling, \& Mazzoni, 1996). Finally, I measured participants' perceptions of the social acceptability of intervention materials and outcomes through a researcher-developed, 9-item, Likert-scale survey. Results of this study show a functional relation for accuracy with answering literal and inferential comprehension questions and for sophistication with applying comprehension thinking strategies to read instructional-level text. All four participants performed within a similar range on on-grade-level probes as compared to instructional-level probes before or after the intervention. Intervention effects maintained at the end of a 2- to 8-week period at a level above respective baseline performance. Participants improved or maintained scores on a standardized achievement test. Moreover, participants' attitudes toward reading and their motivation toward reading increased or maintained at moderately high levels. Results from social validation questionnaires showed favorable impressions of the materials and outcomes. Findings are discussed with regard to the need for future research and the implications for practice. 
EFFECTS OF EXPLICIT READING COMPREHENSION STRATEGY

INSTRUCTION FOR ENGLISH LEARNERS WITH

SPECIFIC LEARNING DISABILITIES

SARA L. JOZWIK

A Dissertation Submitted in Partial

Fulfillment of the Requirements

for the Degree of

DOCTOR OF EDUCATION

Department of Special Education

ILLINOIS STATE UNIVERSITY

2015 
(C) 2015 Sara L. Jozwik 
EFFECTS OF EXPLICIT READING COMPREHENSION STRATEGY INSTRUCTION FOR ENGLISH LEARNERS WITH

SPECIFIC LEARNING DISABILITIES

SARA L. JOZWIK

COMMITTEE MEMBERS:

Yojanna Cuenca-Carlino, Chair

Karen H. Douglas

April L. Mustian 


\section{ACKNOWLEDGMENTS}

I offer sincere gratitude to my family and friends who helped me along this journey. Without your love and support, I would not be where I am today.

I would like to express the deepest appreciation to my committee chair, Yojanna Cuenca-Carlino, Ph.D., whose unwavering support made this accomplishment possible. Without her guidance, this dissertation would not have been possible.

I would like to thank my committee members, Karen Douglas, Ph.D., and April Mustian, Ph. D., whose gave time, care, and insight to add strength to this study. In addition, I offer a special thank you to James Thompson, Ph.D., for pushing me to excel in research and teaching. Your wise conception of a good dissertation had lasting effect.

Finally, I would also like to thank the students, parents, staff members, administrators, and school board members who supported me in carrying out our mission of learning for all -whatever it takes.

S.L.J. 


\section{CONTENTS}

\section{Page}

ACKNOWLEDGMENTS

CONTENTS

ii

TABLES vii

FIGURES viii

\section{CHAPTER}

I. THE PROBLEM AND ITS BACKGROUND 1

Introduction 1

Statement of the Problem $\quad 6$

Purpose 13

Research Questions 14

Definitions of Terms 15

II. REVIEW OF RELATED LITERATURE 21

$\begin{array}{ll}\text { Literature Search Procedures } & 21\end{array}$

Criteria-Based Selection $\quad 23$

Population Validity 23

Students with Specific Learning Disabilities 24

Reading Comprehension Difficulties for Students with Specific

$\begin{array}{ll}\text { Learning Disabilities } & 27\end{array}$

Students Learning English as a New Language 28

Reading Comprehension Difficulties for English Learners 31

Background Knowledge $\quad 31$

Word Reading Ability $\quad 32$

Vocabulary $\quad 32$

Word-Calling $\quad 32$ 
Findings from Major Reviews of Reading Comprehension

Intervention Studies

Prominent Approaches to Improving Reading Comprehension

Reading Comprehension Interventions for English Learners

with Specific Learning Disabilities

Explicit Strategy Instruction with a Self-Monitoring Procedure

Synthesis and Conclusions

$\begin{array}{lll}\text { III. RESEARCH DESIGN } & 74\end{array}$

$\begin{array}{ll}\text { Design of the Study } & 74\end{array}$

Threats to Internal Validity $\quad 75$

$\begin{array}{ll}\text { Criteria for Condition Changes } & 75\end{array}$

$\begin{array}{ll}\text { Participants } & 77\end{array}$

$\begin{array}{ll}\text { Setting } & 81\end{array}$

$\begin{array}{ll}\text { Instructional Materials } & 87\end{array}$

$\begin{array}{ll}\text { Instructional Technology Tools } & 87\end{array}$

Instructional-Level Text $\quad 88$

Assessment to Determine Instructional Reading Levels 88

$\begin{array}{lr}\text { Generalization Texts } & 89\end{array}$

Mentor Texts 90

Prompt Cards for the Reading Comprehension Strategy 91

Self-Monitoring Procedure Cards 91

$\begin{array}{ll}\text { Dependent Measures } & 92\end{array}$

Sophistication with Applying Reading Comprehension Strategies 92

Accuracy with Answering Comprehension Questions 93

Reading Comprehension Achievement 94

Motivation to Read 95

Participants' Perceptions of the Intervention 96

$\begin{array}{ll}\text { Procedures } & 97\end{array}$

$\begin{array}{ll}\text { Collecting Pretest Data } & 97\end{array}$

Baseline Condition $\quad 98$ 
$\begin{array}{ll}\text { Intervention Condition } & 100\end{array}$

Training Phase

Modeling with mentor text

102

Guided practice lessons

Independent Practice Sessions 102

Maintenance Condition

Reliability

Procedural Reliability

Interobserver Reliability

IV. ANALYSIS OF THE DATA

Reading Comprehension Performance with

Sophistication with Applying Reading Comprehension Strategies

Miguel

Maria

Juan

Accuracy with Answering Reading Comprehension Questions

Miguel

Abdul

Maria

Juan

Reading Comprehension Performance with

On-Grade-Level Text

Maintenance of Performance with Instructional-Level Text

Miguel

Abdul

122

Maria

123

Juan 
Reading Comprehension Achievement

Participants' Perceptions of the Intervention

Summary

\section{SUMMARY, CONCLUSIONS, AND} RECOMMENDATIONS

Major Findings

Reading Comprehension Performance with Instructional-Level Text

Comprehension thinking strategy rubric scores

Reading Comprehension Performance with

On-Grade-Level Text

Maintenance of Performance with Instructional-Level Text

Reading Comprehension Achievement

Participants' Perceptions of the Intervention

Limitations and Implications for Future Research

APPENDIX A: Rubric for Culturally Responsive Research Trainor and Bal (2014) 169

APPENDIX B: Comprehension Thinking Strategy Rubric 
APPENDIX I: Procedural Reliability Checklist for Baseline and Maintenance Conditions

APPENDIX J: Procedural Reliability Checklists for Intervention Lessons

APPENDIX K: Explicit Instruction Checklist

APPENDIX L: Scripted Intervention Lesson Plans

APPENDIX M: List of Culturally Relevant Texts 


\section{TABLES}

Table $\quad$ Page

1. Attributes of Effective Literacy Interventions for ELs with Learning Difficulties

2. Key Information from Reading Comprehension Intervention Studies

3. Participant Characteristics

4. Teaching Actions During Baseline and Maintenance Conditions

5. Sequence of Lessons During the Intervention Condition

6. Procedural Fidelity Data

7. Interobserver Agreement Data

8. Comprehension Thinking Strategy Rubric Scores for Instructional-Level Text

9. Accuracy with Answering Comprehension Questions on Instructional-Level Text

10. Reading Comprehension Performance with On-Grade-Level Text

11. Reading Comprehension Achievement Scores

12. Motivation to Read Survey Results

13. Participants' Perceptions of the Intervention 


\section{FIGURES}

$\begin{array}{lll}\text { Figure } & \text { Page }\end{array}$

1. Underlying Causes of Reading Difficulties for ELs and Students with SLD 3

2. Self-Monitoring Procedure Card 92

3. Sophistication with Applying Reading Comprehension Strategies Across Sessions

4. Accuracy with Answering Comprehension Questions Across Sessions 


\section{CHAPTER I}

\section{THE PROBLEM AND ITS BACKGROUND}

\section{Introduction}

A distinguishing characteristic of the United States today is the linguistic diversity of its population. More than four and a half million children in U.S. public schools speak a native language other than English (National Center for Education Statistics [NCES], 2013). This reflects a $40 \%$ increase in the number of school-age, non-native English speakers over the past 30 years (National Clearinghouse for English Language Acquisition, 2011). Linguistic differences (i.e., having limited English proficiency) are associated with challenges (e.g., lower socioeconomic status) that ultimately contribute to making language status an at-risk factor for the $9.1 \%$ of U.S. public school students who are learning English as a second or additional language (NCES, 2013). Having limited English proficiency affects academic achievement in a direct way: Students who come to school with limited English proficiency have greater difficulty learning to read in English than their monolingual, native English-speaking peers (Abedi, 2002; Freeman \& Freeman, 2002). Nationwide, disaggregated data on achievement outcomes reveal disparities between English Learners (ELs) — students who are in the process of acquiring English as a new language_-and English-only students.

On the 2013 National Assessment of Educational Progress (NAEP) in fourthgrade reading, ELs achieved average scale scores $(\mathrm{SS}=187)$ that fell 38 points below 
English-only peers' scores (SS $=225$ ), with $69 \%$ of ELs and $28 \%$ of English-only students reading below a basic level. In eighth grade, on the 2013 NAEP, the achievement gap in reading widened to a 45-point difference between average scale scores earned by ELs as compared to English-only students, with 70\% of ELs and 20\% of English-only students reading below a basic level (NCES, 2013). Lack of Englishreading proficiency has grave short- and long-term ramifications for ELs (Biancarosa \& Snow, 2006). In the short term, access to general education curriculum is restricted, and academic achievement is adversely affected. Over the long term, ELs experience grade retention at higher rates, are twice as likely to drop out of high school, and are more likely to have limited employment opportunities as compared with English-only peers (August \& Hakuta, 1997; August \& Shanahan, 2006; Snow, Burns, \& Griffin, 1998; Snyder \& Dillow, 2012).

As the country's EL population expands, more ELs are identified, or misidentified, with specific learning disabilities (SLD). Researchers acknowledge that the population of ELs who are identified with SLD has increased at a disproportionate rate (Harry \& Klingner, 2012). Disproportionality refers to "the extent to which membership in a given group affects the probability of being placed in a specific disability category (Oswold, Coutinho, Best, \& Singh, 1999, p. 198). Among the schoolage population of native English speakers in American public schools, roughly 5\% of students are identified with SLD under the Individuals with Disabilities Education Act [IDEA, 2004] (Cortiella \& Horowitz, 2014). By contrast, among the school-age population of non-native English speakers, an estimated 16\% are identified with SLD 
(Albus \& Thurlow, 2007). Ultimately, due to difficulties with discerning difference from disability, overidentification of ELs with SLD is a nationally documented reality (Donovan \& Cross, 2002; Samson \& Lesaux, 2009).

For many ELs, the language acquisition process is often misinterpreted and misidentified as SLD, and the students' primary educational needs are found in the area of literacy (Artiles \& Ortiz, 2002; Klingner, Artiles, \& Méndez Barletta, 2006). Indeed, it is difficult to disambiguate the challenges associated with limited English proficiency from those associated with having SLD. In the area of literacy, several behaviors associated with second language acquisition (e.g., poor reading fluency, difficulty with reading sight words, and difficulty with retelling a story) overlap with behaviors demonstrated by students who have SLD. This overlap is depicted in Figure 1. Although overt behaviors appear to be quite similar, analysis of underlying, causal factors allows clearer distinctions to be made between a learning difficulty and a language difference.

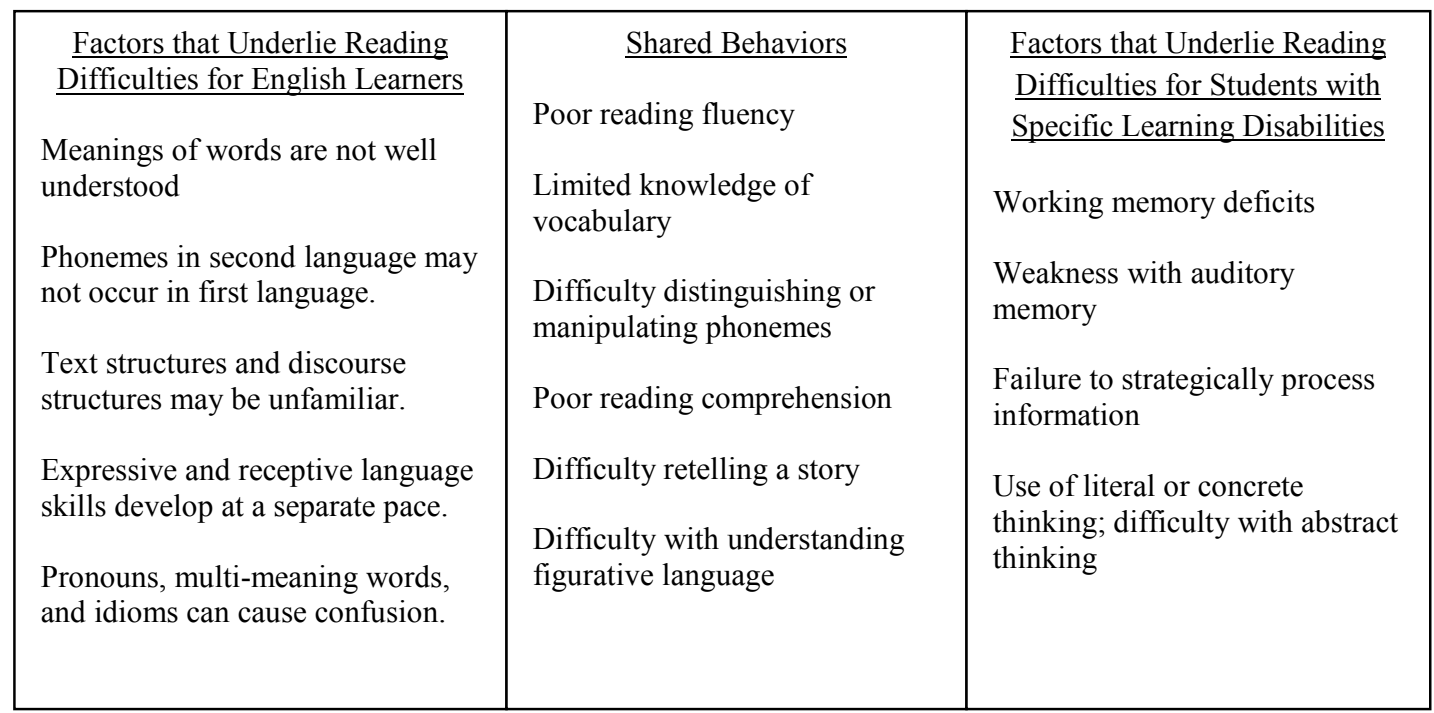

Figure 1. Underlying causes of reading difficulties for ELs and students with SLD. 
An initiative set forth by the No Child Left Behind (NCLB) Act and the Individuals with Disabilities Education Act (IDEA), known as Response to Intervention (RTI), holds the potential to reduce the over-identification of ELs for special education services under the SLD eligibility category (Rinaldi, 2011). The RTI initiative has the potential to effect positive change by requiring the use of research-based practices to address an individual's specific learning needs. This is done through systematic documentation of the student's response to the intervention. When a lack of progress is depicted in the data, adjustments to the intervention must be made prior to making a referral for a special education evaluation (Brown \& Doolittle, 2008). It is important to acknowledge, however, that few research-based approaches for improving reading have been validated for ELs (Klingner \& Eppolito, 2014).

Under the IDEA, determining that an EL has an SLD involves establishing that (a) the student's learning difficulties are not primarily the result of language acquisition and (b) that the student has had an adequate opportunity to learn through research-based instructional and intervention practices that have been validated with other ELs of a similar language background at a similar level of language proficiency. Faced with challenges such as a limited research base (i.e., most studies focus on low-level literacy skills), lack of empirically validated instructional resources, lack of adequately trained personnel (i.e., without bilingual special education credentials), and limited understanding of typical literacy development in second language learners, practitioners are left to use intervention practices that have been validated for monolingual, native 
English speakers during the RTI and special education referral process (Klingner \& Eppolito, 2014).

Interventions that have been found to be effective for English-only struggling readers are inadequate for ELs who struggle in reading (Klingner, Boelé, LinanThompson, \& Rodriguez, 2014). To be effective for ELs, interventions must support language acquisition and demonstrate cultural responsiveness while delivering targeted, academic instruction to address the student's areas of need (Klingner \& SolteroGonzález, 2009; Ortiz, Wilkinson, Robertson-Courtney, \& Kushner, 2006). Since the NCLB Act was signed into law in 2002, reading has become an area for which many instructional interventions have been designed, implemented, and tested. Current research on effective literacy interventions for ELs focuses mainly on early literacy skills (e.g., alphabetic principle, phonemic awareness, phonics, and automatic word recognition). However, beginning at the upper-elementary grade levels, reading instruction focuses on the development of reading comprehension skills and strategies that will allow students to reach the goal of reading for meaning across content areas. The research base to guide practitioners in improving reading comprehension for ELs with SLD is limited to twelve studies (i.e., Bos \& Anders, 1992; Denton, Wexler, Vaughn, \& Bryan, 2008; Graves, Duesbery, Pyle, McIntosh, \& McIntosh, 2011; Gunn, Smolkowski, Biglan, \& Black, 2002; Jiménez, 1997; Klingner \&Vaughn, 1996; LinanThompson, Vaughn, Hickman-Davis, \& Kouzekanani, 2003; McElvain, 2010; Sáenz, Fuchs, \& Fuchs, 2005; Santoro, Jitendra, Starosta, \& Sacks, 2006; Vaughn, Mathes, \& Linan-Thompson, 2006; Wanzek \& Roberts, 2012). 
When reading comprehension is identified as an area of need after a special education evaluation is complete, there is a paucity of high-quality research that can inform practitioners on how best to intervene for an EL with SLD who struggles with reading comprehension. Without empirically based, culturally responsive literacy intervention practices, the negative long-term outcomes dually associated with EL and SLD labels will persist (Klingner \& Edwards, 2006; Orosco \& Klingner, 2010).

Ultimately, more research on effective reading comprehension interventions is needed to ensure that ELs with SLD can access the general curriculum and experience success in school and life (Thorius \& Sullivan, 2013).

\section{Statement of the Problem}

English Learners, by virtue of having limited English proficiency, experience difficulties with reading and extracting meaning from text that is written in English. Experiencing success in school hinges on activating reading comprehension skills to read various texts across content areas (Edmonds et al., 2009). As early as third grade, the struggle to construct meaning from on-grade-level text jeopardizes ELs' access to instructional material across content areas. To prevent the perpetuation of the achievement gap and to meet language learning and academic needs of ELs, specific language-learning supports (e.g., visuals, gestures, and demonstrations) need to be put in place across content areas (Klingner, Boardman, Eppolito, \& Schonewise, 2012). When an EL has a disability that further compounds his or her struggle to read for meaning (e.g., dyslexia), he or she requires an intensified level of support that is responsive to 
individual needs and that takes into account important considerations for supporting literacy development in a second or additional language.

To support literacy development for ELs, it is important to consider the language of instruction, the opportunities for oral language development, and the affective factors associated with learning a new language. Current research confirms that ELs with SLD, emotional disturbance, or speech language impairment will experience challenges (e.g., difficulty regulating emotions, deficits in working memory, or expressive language deficits) when using both the home language and English (Simon-Cereijido \& GuitiérrezClellen, 2014). For interventionists, the optimal response is to continue using both languages rather than restricting input to only one language (Paradis, Genesee, \& Crago, 2011). Professionals often assume that to acquire English, students with disabilities need to spend more time receiving instruction in English only and consequently minimize exposure to the students' native languages. This assumption holds intuitive appeal; however, according to empirical evidence on cross-linguistic transfer, for students with and without disabilities, any amount of native language instruction produces greater gains in performance on standardized tests in English compared with no native language instruction (August \& Hakuta, 1997; August \& Siegel, 2006; Slavin \& Cheung, 2005). When delivering an academic intervention to target literacy, the language of intervention should match the language of classroom reading instruction while offering home language support (e.g., translating vocabulary into the native language to clarify meanings of unknown words) to promote second language acquisition (Klingner et al., 2014; Ortiz, 2001). 
Klingner and Soltero-González (2009) noted that, once placed into special education programs, ELs are less likely than their EL peers without disabilities to receive any amount of support in second language acquisition and that they are more likely to be instructed only in English. Given the academic gains associated with native language support, parents or caregivers should be encouraged to engage their children in literacyrelated activities in the home language (Wong Fillmore, 2000). At school, strategic use of the home language not only assists ELs in acquiring literacy and accessing content, but also it reflects cultural responsiveness and thereby plays a role in facilitating strong home-school partnerships (Kalyanpur \& Harry, 2012).

In rare cases where no amount of literacy support can be provided in the native language, strategies for teaching English as a second language (ESL) should be incorporated into instruction. The use of demonstrations, realia (i.e., replicas or real life objects), and gestures are strategies that ESL teachers use to support students' understanding of verbal and written messages (Peregoy \& Boyle, 2008). In addition, ESL teaching strategies include: setting clear language and learning objectives; modeling language use; providing frequent opportunities for practice with feedback in the domains of reading, writing, listening, and speaking; engaging students in active, hands-on participation throughout lessons; and using various cooperative learning structures (Linan-Thompson \& Vaughn, 2007; Peregoy \& Boyle, 2008). ESL teaching strategies align with and can be easily integrated into lessons that satisfy the Institute of Education Sciences' top three recommendations for effective literacy instruction for ELs: (a) provide intensive and explicit instruction (e.g., providing modeling, guided practice, and 
opportunities for independent practice), (b) conduct frequent formative assessments (e.g., by checking for understanding and by providing feedback on performance in all language domains), and (c) provide high-quality and extensive vocabulary instruction (e.g., by allowing cooperative practice with social and academic language structures) (Gersten, Baker, Shanahan, Linan-Thompson, Collins, \& Scarcella, 2007).

In cases where native language support is feasible, ESL teaching strategies should also be incorporated into instruction to ensure messages are comprehensible to ELs. Regardless of the student's native language or the feasibility of translation, messages communicated in English to ELs must be delivered at a level slightly in advance of their current levels of language proficiency (Linan-Thompson \& Vaughn, 2007). Krashen (1981) proposed this concept, which is referred to as comprehensible input+1. In keeping with recommended practices for literacy instruction for ELs, comprehensible input +1 involves delivering messages in conjunction with gestures, visual supports, demonstrations, and frequent formative assessments.

In addition to providing native language support and comprehensible input in English, opportunities for practice with oral language must be created. Oral language development is an essential component of second language literacy development (August $\&$ Shanahan, 2006). Oral language reinforces and is reinforced by literacy through a reciprocal relationship. Additionally, ELs need oral language support to develop knowledge of specialized vocabulary, specific phrases or sentences, and academic discourse patterns (Bailey, 2007). For ELs with SLD, frequent opportunities to engage in oral language practice can improve oral language development, which, ultimately, 
correlates with improved reading ability (Genesee, Lindholm-Leary, Saunders, \& Christian, 2005; Gentile, 2004; Geva \& Massey-Garrison, 2013). Using cooperative learning structures (e.g., think-pair-share) and providing sentence frames (e.g., "I predict that this story will be about .") facilitates the opportunity for increased use of oral language during instruction (Gentile, 2004). A distinction should be made, however, between providing opportunities to use oral language and providing motivation for ELs to develop academic and social oral language. Indeed, motivation levels affect learning and language development (Snow, Burns, \& Griffin, 1998).

Effective literacy instruction for ELs recognizes and supports affective factors related to language learning. Learning in a second language while still in the process of acquiring the language can be a frustrating experience (Klingner \& Eppolito, 2014). Frequent errors, although a viable sign of language learning, can compound an EL's frustration with feelings of anxiety or embarrassment. By reflecting on or conducting an inventory of the learning environment, teachers or interventionists can become aware of affective, sociocultural, and other contextual factors (e.g., interpersonal dynamics of the classroom) that affect learning. This process and its inherent self-examination allows for steps to be taken to reduce or eliminate potential threats to student learning (e.g., by using a turn-and-talk rather than calling on individual students to answer questions orally; Ortiz et al., 2006). In addition to creating an environment where ELs feel safe in taking risks to use the English language, literacy interventions must also provide meaningful, interesting, and relevant instruction to ignite student learning (Damico, \& Nelson, 2010; Rueda, MacGillivary, Monzó, \& Arzubiaga, 2001). To engage ELs in a literacy 
intervention aimed at improving reading comprehension, additional actions can lead to increased student engagement. Three actions in particular reflect cultural responsiveness and can increase student motivation during literacy instruction: considering students' background experiences while making text selections, including authors from a variety of cultures, and giving students voice and choice in their learning goals (Klingner \& Soltero-González, 2009).

ELs require support to acquire proficient reading skills in English. Once the presence of SLD has been identified, high-quality research is needed to: (a) illustrate how interventions can be individualized in a way that reflects cultural responsiveness, (b) support language-learning needs of ELs, (c) increase students' access to the general curriculum, and (d) improve reading achievement. ELs with SLD have a unique set of linguistic, cultural, and academic needs. Because unique needs impact learning and achievement, interventions that aim to remediate reading difficulties must take these needs into account.

An assumption is commonly made that what works for native English speakers with SLD will also work for ELs with SLD. In addition to the general considerations discussed above (i.e., providing native language support, embedding opportunities for oral language development, and taking into account affective factors), within each area of literacy (i.e., phonemic awareness, phonics, vocabulary, fluency, comprehension, and writing) interventions designed for native English speakers with SLD are inadequate and require modification in order to be effective for ELs with SLD (Klingner \& SolteroGonzález, 2009). Specific modifications that can transform interventions and contribute 
to positive effects for ELs with learning difficulties (i.e., attention deficit/hyperactivity disorder, emotional disturbances, or SLD) are described relative to knowledge claims in the extant research for each area of literacy in Table 1.

Table 1

Attributes of Effective Literacy Interventions for ELs with Learning Difficulties

\begin{tabular}{|c|c|}
\hline Areas of Focus & Attributes \\
\hline General & 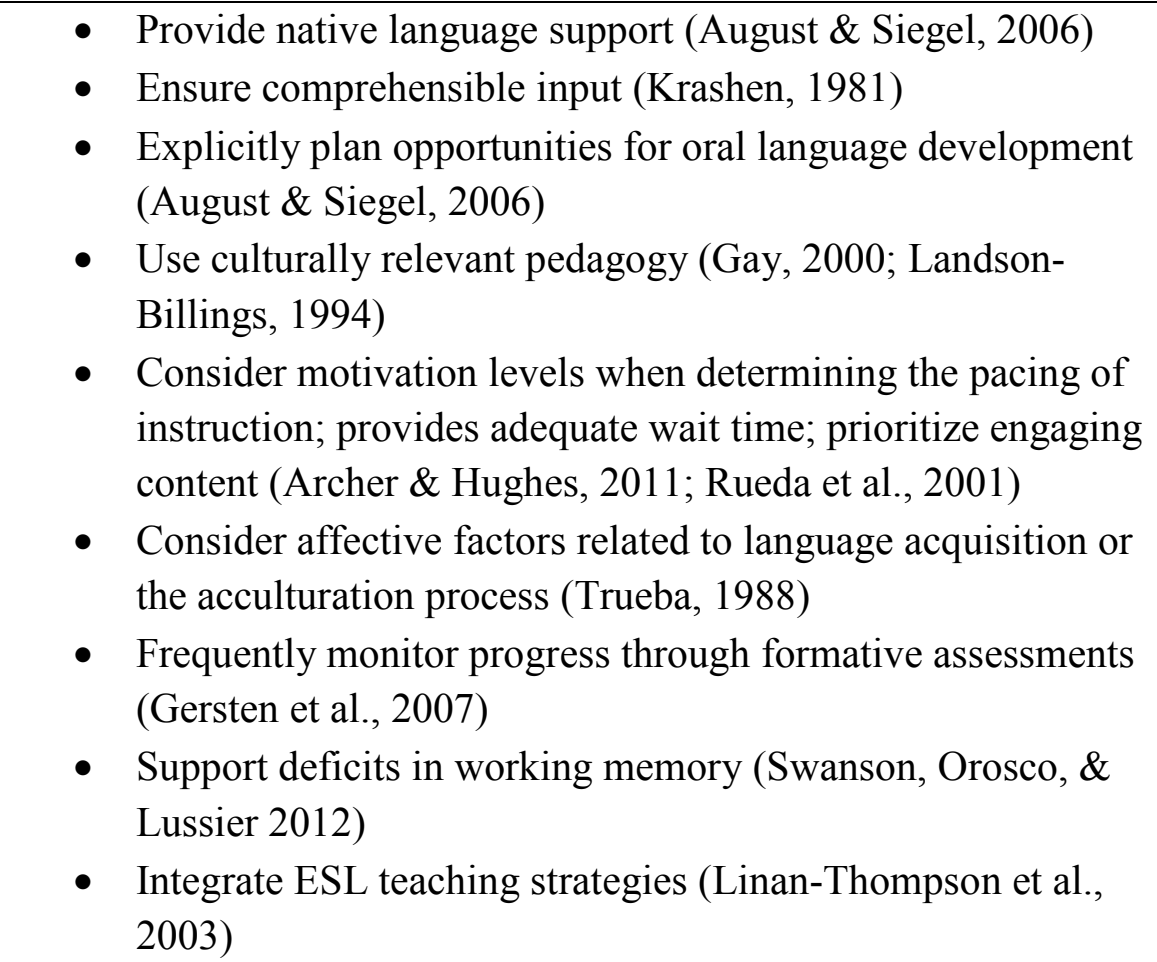 \\
\hline $\begin{array}{l}\text { Phonemic } \\
\text { awareness }\end{array}$ & $\begin{array}{l}\text { - Provide explicit instruction to help distinguish and } \\
\text { pronounce sounds not in the home language (Kress, 2008) } \\
\text { - Present practice with minimal pairs (e.g., discriminating /b/ } \\
\text { from /v/ in "ban" and "van") }\end{array}$ \\
\hline Phonics & $\begin{array}{l}\text { - Ensure a balanced emphasis between word identification and } \\
\text { word comprehension (Klingner \& Eppolito, 2014) } \\
\text { - Incorporate sounds and words that are familiar to students } \\
\text { (Klingner \& Eppolito, 2014) }\end{array}$ \\
\hline
\end{tabular}

(Table Continues) 


\begin{tabular}{|c|c|}
\hline Areas of Focus & Attributes \\
\hline Fluency & $\begin{array}{l}\text { - Acknowledge that when comprehension increases, reading } \\
\text { rate decreases (Crosson \& Lesaux, 2009) } \\
\text { - Provide opportunities for repeated reading (O'Connor, } \\
\text { White, \& Swanson, 2007) } \\
\text { - Offer support by pre-teaching vocabulary or key ideas for } \\
\text { which students lack schema (Tam, Heward, \& Heng, 2006) }\end{array}$ \\
\hline Vocabulary & $\begin{array}{l}\text { - Focus on content-area terms in addition to text-specific terms } \\
\text { (Snow, Lawrence, \& White, 2009) } \\
\text { - Use explicit instruction in conjunction with strategies to } \\
\text { meaningfully engage students in learning new words, such as } \\
\text { self-regulation strategies (Helman, Calhoon, \& Kern, 2014) }\end{array}$ \\
\hline Comprehension & $\begin{array}{l}\text { - Teach metacognitive strategies (Jiménez, 1997) } \\
\text { - Contextualize strategy instruction rather than providing } \\
\text { isolated skill-drill practice (Lesaux \& Harris, 2013) } \\
\text { - Incorporate frequent and extended opportunities to practice } \\
\text { using language in oral and written forms (Bos \& Anders, } \\
\text { 1992) }\end{array}$ \\
\hline Writing & $\begin{array}{l}\text { - Incorporate modeled writing, guided writing, interactive } \\
\text { writing, and collaborative writing } \\
\text { - Provide explicit strategy instruction (De La Paz \& Sherman, } \\
\text { 2013) }\end{array}$ \\
\hline
\end{tabular}

\section{Purpose}

This study aimed to replicate and extend previous studies (Jiménez, 1997;

Jitendra, Hoppes, \& Xin, 2000) on reading comprehension strategy instruction.

Specifically, this study evaluated the effectiveness of a reading comprehension intervention for ELs with SLD. The intervention incorporated explicit instruction in applying reading comprehension strategies and used a transfer-promoting procedure to teach participants to self-monitor use of three reading comprehension strategies: 
monitoring comprehension, using schema, and questioning. First, the intervention replicated Jiménez's (1997) use of teacher-mediated metacognitive strategy instruction that incorporated culturally relevant text and provided native language support to meet needs of ELs with learning difficulties. Whereas Jiménez provided one-to-one instruction to participants in a self-contained special education setting, I provided instruction to small, heterogeneous groups of students in a general education setting where special education services were delivered through co-teaching. Second, the intervention extended upon the findings of Jitendra et al. (2000) by providing reading comprehension strategy instruction and using a similar self-monitoring procedure to examine intervention effects for ELs with SLD and to monitor participants' performance with the comprehension strategies and self-monitoring procedures through formative assessments to ensure mastery prior to measuring effects on generalization and maintenance. The study addressed the need for more research on how to improve reading comprehension for ELs with SLD while also providing a model of how principles of culturally responsive research can be integrated into intervention research.

\section{Research Questions}

1. What are the effects of explicit reading comprehension strategy instruction when combined with a self-monitoring procedure on participants' application of comprehension strategies during close reading of instructional-level text?

2. What are the effects of explicit reading comprehension strategy instruction when combined with a self-monitoring procedure on participants' accuracy with 
answering researcher-developed literal and inferential comprehension questions after reading instructional-level text?

3. To what extent are participants able to generalize reading comprehension performance (i.e., application of comprehension strategies and accuracy with answering literal and inferential questions) while reading on-grade-level text?

4. To what extent are participants able to maintain reading comprehension performance (i.e., application of comprehension strategies and accuracy with answering literal and inferential questions) with instructional-level text?

5. To what extent do effects of explicit reading comprehension strategy instruction transfer from formative assessments to standardized measures of reading comprehension achievement?

6. How does participation in an intervention that offers explicit comprehension strategy instruction combined with a self-monitoring procedure affect motivation toward reading and self-concept as a reader?

7. How do participants perceive the usefulness of explicit reading comprehension strategy instruction with a self-monitoring procedure?

\section{Definitions of Terms}

Close reading. Attending closely to both one's own schema and the information presented in the text. Beers and Probst (2013) described close reading as a process that brings the reader closer to the text, thereby creating space for relevance, engagement, and rigor. Close reading skills are developed through four simultaneous behaviors: "close attention to the text; close attention to the relevant experience, thought, and memory of 
the reader; close attention to the responses and interpretations of other readers; and close attention to the interactions among these elements" (Beers \& Probst, 2013, p. 37).

Comprehensible input. Language input that can be understood even when a listener does not understand all of the words and structures used to construct the message. To allow a listener to grasp the essence of a message, the speaker may use demonstrations, gestures, visual supports, graphic organizers, and carefully controlled vocabulary.

Cross-linguistic transfer. Cross-linguistic transfer is evidenced across languages (e.g., Japanese to English) based on Cummins' (1979) interdependence hypothesis. Evidence (Genessee, 1978; Geva, 2000) supports this hypothesis by showing that knowledge from one language will transfer to the new language. Depending on the sociolinguistic situation, cognate languages (e.g., Spanish and English) and dissimilar languages (e.g., Arabic and English) share features that establish a common underlying proficiency. In cognate languages, shared features include linguistic concepts (e.g., letters and phonemes). Conceptual features (e.g., pragmatics, metacognition) are shared among cognate languages and dissimilar languages. According to Lado’s (1964) contrastive analysis hypothesis, cross-linguistic transfer is more likely to occur when the speaker perceives similarity between languages. Ultimately, transfer can facilitate second language development, but it is not the sole source of influence on second language development.

Culturally responsive instruction. Culturally responsive teaching, or culturally relevant pedagogy, is described as "a pedagogy that empowers students intellectually, 
socially, emotionally, and politically by using cultural referents to impart knowledge, skills, and attitudes" (Landson-Billings, 1994, p. 382). This entails finding out what appeals to students, giving students a voice, transmitting instruction in their language (i.e., more than merely translating words), and making standards-based curricula accessible to all students.

Culturally responsive research. According to Trainor and Bal (2014), research can be viewed as a situated cultural practice. From inception to dissemination, research is "culturally and socially mediated and negotiated" (Arzubiaga, Artiles, King, \& HarrisMurri, 2008, p. 310). Culturally responsive research "acknowledges power and inequity as central players in the reproduction of educational disparities" and asserts that results can be understood only when "the physical, sociocultural, and historical contexts of the researchers and the participants frame the work" (Trainor \& Bal, 2014, p. 47; see Appendix A -Rubric for Culturally Responsive Research).

English Learner. A term used to describe a student with limited English proficiency. A student in a U.S. public school is considered an English Learner when specific criteria are met. First, at the time of school registration, the parent or guardian must report that the student lives in a home where a language other than English is spoken and must report that the student uses a language other than English. Second, school personnel must determine that the student is in the process of acquiring English as a new language by examining results from an initial English language proficiency screener and by administering and scoring an annual English language proficiency assessment. 
Explicit instruction. A direct, systematic, and effective method for teaching academic skills. Using a direct approach (where nothing is left implicit), "students are guided through the learning process with clear statements about the purpose and rationale for learning the new skill, clear explanations and demonstrations of the instructional target, and supported practice with feedback until independent mastery has been achieved" (Archer \& Hughes, 2011, p.1).

Learning difficulties. This term is used to refer to challenges that affect learning, particularly when students are found eligible to receive special education services under the following categories: specific learning disability, emotional disturbance, or other health impairment due to a diagnosis of attention deficit/hyperactivity disorder.

Reading comprehension. An interactive process that involves the activation of the reader's prior experience and knowledge about the world and about language. It entails using strategies such as predicting, questioning, summarizing, determining meanings of vocabulary in context, monitoring one's own comprehension, and reflecting. The process also engages such affective factors as motivation, ownership, purpose, and self-esteem. It is governed by specific context, and it is dependent on social interaction. The integration of all these processes contributes to the conception of reading comprehension as a holistic process for constructing meaning (Bartoli \& Botel, 1998).

Reading comprehension achievement. Results from pretest to posttest on a valid and reliable standardized assessment (i.e., the passage comprehension subtest of the Woodcock Johnson Tests of Achievement III -R; Woodcock, McGrew, \& Mather, 2001) 
that yields a standard score, percentile rank, and grade level equivalency score to indicate achievement in reading comprehension.

Reading comprehension performance. Reading comprehension performance refers to performance with tasks that require application of reading comprehension strategies, as measured through formative assessments. Formative assessments measured (a) application of targeted comprehension thinking strategies while using close reading to make meaning from selected passages, as assessed through Keene's (2006) reading comprehension thinking strategy rubrics (see Appendix B -Comprehension Thinking Strategy Rubric) and (b) accuracy with answering literal and inferential comprehension questions as determined through verbal responses to researcher-developed text-dependent questions (see Appendix C-Comprehension Question Frames).

Reading comprehension strategies. Proficient readers use thinking strategies to make sense of text when they read. Harvey and Goudvis (2008) describe these strategies as: determining importance, drawing inferences, using prior knowledge, asking questions, monitoring comprehension, summarizing, synthesizing, and creating mental images. The use of the strategy monitoring comprehension was the main focus of this study. This strategy subsumes several of the strategies described by Harvey and Goudvis. To monitor for meaning, students use a variety of strategies that include: paying attention to the inner voice to focus thinking; listening to the inner voice and leaving tracks of thinking by coding text; identifying confusions and using fix-up strategies like looking back or re-reading confusing parts, reading ahead to clarify confusions, taking a break, 
connecting what is known to what is read in the text, asking questions, or talking to someone who knows a lot about the topic.

Scientifically-based instruction. Instructional strategies based on research that (a) employed empirical methods and rigorous data analyses; (b) used experimental or quasiexperimental designs; (c) allowed for replication; (d) was accepted by peer reviewed journals or have been reviewed and approved by independent, expert panels. In addition, instructional strategies must have been proven effective in addressing the specific issues that resulted in the need for improvement (Ortiz \& Yates, 2008, pp. 13-14).

Self-monitoring procedure. A sequence of four steps, presented in checklist form, for monitoring use of reading comprehension strategies. The checklist contains four statements that communicate a sequence of steps to follow. The four steps are: (a) I read the paragraph; (b) I used the prompt card to recall the strategies; (c) I applied strategies to monitor for meaning; and (d) I coded the text to leave tracks of my thinking.

Specific Learning Disability. A disorder in one or more of the basic psychological processes involved in understanding or in using language, spoken or written, that may manifest itself in an imperfect ability to listen, think, speak, read, write, spell, or to do mathematical calculations, including conditions such as perceptual disabilities, brain injury, minimal brain dysfunction, dyslexia, and developmental aphasia. Specific learning disability, under the IDEA, does not include learning problems that are primarily the result of visual, hearing, or motor disabilities; intellectual disability; emotional disturbance; environmental factors; cultural differences; limited English proficiency; or economic disadvantage. 


\section{CHAPTER II}

\section{REVIEW OF RELATED LITERATURE}

This chapter presents an overview of the literature on English Learners (ELs) with specific learning disabilities (SLD) as it relates to both performance with applying reading comprehension strategies and reading comprehension achievement. The first section describes attributes of students with SLD and examines how breakdowns in reading comprehension occur for students with SLD. The second section describes attributes of students who are learning English as a new language and examines how breakdowns in reading comprehension occur for ELs. Next, findings from major reviews of reading comprehension intervention studies are discussed. This discussion is followed by a description of prominent approaches for improving reading comprehension. The fifth section addresses the combination of explicit instruction in reading comprehension strategies with self-monitoring procedures. Finally, the chapter concludes with a summary and synthesis of the literature on reading comprehension strategy instruction for ELs with SLD.

\section{Literature Search Procedures}

I conducted a comprehensive search of the literature using four methods: (a) keyword searches in subject indexes, (b) browsing, (c) ancestral searches, and (d) consultation. First, I conducted computer searches of the following online databases: Academic Search Complete, Education Full Text, Educational Resources Information 
Center (ERIC), ProQuest (dissertation databases), Psyc Info, and SAGE Journals. I used the following descriptors and keywords to locate unpublished dissertations or articles published in peer-reviewed journals pertaining to reading comprehension strategy instruction for linguistically diverse students who were struggling in reading or who had been identified with SLD: language minority student, English language learner, limited English proficient, English as a second language, linguistically diverse, at-risk reader, struggling reader, learning disabilities, reading disability, reading comprehension, and intervention. In addition, I used truncation of the following terms: comprehen*, learning disab*, and reading disab* to capture the greatest possible number of empirical studies. The second step involved a hand search of 14 refereed journals. I examined the following journals: Annals of Dyslexia, Bilingual Research Journal, Elementary School Journal, Exceptional Children, Journal of Educational Psychology, Journal of Learning Disabilities, Journal of Literacy Research, Journal of Special Education, Learning Disability Quarterly, Learning Disabilities Research and Practice, Reading Research Quarterly, Reading and Writing Quarterly, Remedial and Special Education, and TESOL Quarterly. I first searched within each journal using key terms and then searched through the tables of contents of each issue of each journal from January 1, 2004 to August 5, 2014.

The third step involved searching the reference lists and footnotes from relevant studies to locate additional articles that did not emerge from the first two methods of searching. Also, I attempted to locate studies by contacting researchers who frequently published studies on ELs who struggle in reading. I sent electronic messages asking if 
they had any articles on reading comprehension interventions for linguistically diverse students in progress or in press, or if they were aware of any other researchers with studies in progress.

\section{Criteria-Based Selection}

To determine which articles to include in the review, I established three criteria. I opted to include only empirical studies that: (a) concentrated on a K-12 population in the United States, (b) provided a reading comprehension intervention, and (c) included students with SLD or learning difficulties who were struggling readers and who were in the process of acquiring English as an additional language.

\section{Population Validity}

The second part of the final criterion (i.e., inclusion of ELs with reading difficulties) foments the issue of population validity. Many terms are used to describe students in U.S. public schools who speak a home language other than English (e.g., limited English proficient, language minority, English language learners). Likewise, descriptions of language status vary considerably across studies. For example, some researchers (Calhoon, Otaiba, Greenberg, King, \& Avalos, 2007) used racial or ethnic descriptors combined with a geographic location (e.g., Hispanic population near the Mexico border) to describe language status. Other researchers (Bos \& Anders, 1992: Denton, Anthony, Parker, \& Hasbrouck, 2004) used the term bilingual to describe students who spoke a home language other than English and who were still in the process of acquiring English as a second language. Some researchers used the term English as a second language (ESL) students (Klingner \& Vaughn, 1996) or the term limited English 
proficient (LEP) students (Wanzek \& Roberts. 2012). Most commonly, researchers used the terms English learner or English language learner (Linan-Thompson et al., 2003; Solari \& Gerber, 2008) to indicate that participants were not fully proficient in English.

The lack of consistent terminology leaves the question of whether the targeted population is defined by the same criteria (Moore \& Klingner, 2012). I included studies for which I could confirm (i.e., through analyzing participants' reported English language proficiency test scores, through authors' acknowledgement of participants' classification as limited English proficient according to locally defined criteria, or by directly contacting researchers) that participants used a home language other than English and that participants demonstrated limited proficiency with reading, writing, listening, and speaking in English. Stringency on this criterion is essential for determining the extent to which results can generalize for ELs with reading difficulties.

\section{Students with Specific Learning Disabilities}

From its inception, the field of special education has conceived of specific learning disabilities (SLD) as unexpected underachievement, but difficulty with exacting an operational definition to identify SLD has remained a persistent challenge (Fletcher, Lyon, Fuchs, \& Barnes, 2007). Unexpected underachievement is perceived when struggles to read, write, or do mathematics occur in the absence of conditions (e.g., sensory disorders) that interfere with academic skills. This conceptual understanding relies on exclusionary criteria to define SLD by what it is not (e.g., economic disadvantage or intellectual disability). Over the course of several decades, efforts to identify inclusionary criteria to define SLD have shifted from identifying the presence of 
a neurological disorder, to determining the existence of a cognitive discrepancy, to, ultimately, documenting a student's inadequate response to instruction. Current legislation (IDEA, 2004) defines SLD as

a disorder in one or more of the basic psychological processes involved in understanding or in using language, spoken or written, that may manifest itself in an imperfect ability to listen, think, speak, read, write, spell or to do mathematical calculations, including conditions such as perceptual disabilities, brain injury, minimal brain dysfunction, dyslexia, and developmental aphasia. CFR § 300.8 I

This does not include learning problems that are primarily the result of visual, hearing, or motor disabilities; intellectual disability; emotional disturbance; environmental factors; cultural differences; limited English proficiency; or economic disadvantage.

Students who are identified with SLD manifest challenges with learning in various ways. One common characteristic among individuals with SLD is uneven development or a unique pattern of individualized strengths and weaknesses in the areas of psychological processing (Lerner, 2003). For example, a student may have difficulty decoding words, which leads to challenges with reading connected text; however, when listening to the text read aloud, he or she may be able to answer literal and inferential questions about the story with a high level of accuracy.

Another characteristic associated with SLD involves limitations with working memory, the part of temporary memory that remains active until a task is completed (Berninger \& Swanson, 2014). Efficient working memory allows for skills such as 
handwriting or decoding to become automatized and has been found to support goaldirected behavior (Goldman-Rakic, 1992). Weaknesses in working memory are associated with lack of automaticity and poor planning.

Furthermore, many students with SLD lack organizational skills and fail to mobilize cognitive strategies for learning. This results in weak orientation to tasks, such as reading and comprehending an on-grade-level text. In some cases, SLD co-occurs with existing attention deficit disorders (Lerner, 2003). Organization skills and strategic approaches to task completion are essential for school success.

Finally, students with SLD may also demonstrate difficulties with problemsolving or social skills (Swanson \& Malone, 1992). This may involve difficulties in reading nonverbal cues and difficulties with using pragmatic language. Students with SLD may have never learned the social or cognitive skills necessary for particular social situations. Alternatively, students with SLD may have learned necessary cognitive or social skills but fail to perform them in the appropriate situation. According to teacher reports, reports from peers, and reports from students themselves, students with SLD experience greater social skill deficits than peers without SLD (Kavale \& Forness, 1996). In summary, many characteristics associated with SLD result in difficulties that impact academic achievement in one or more area (e.g., mathematics, writing, reading, or oral language). Given the intra-individual heterogeneity associated with SLD, the approaches or solutions that are applied to address SLD are adaptive and flexible. These approaches include using direct, systematic instruction and using learning strategies (Lerner, 2003). 


\section{Reading Comprehension Difficulties for Students with Specific Learning Disabilities}

Many students with learning, attention, or emotional disabilities experience difficulties with reading acquisition, particularly with comprehending written material (Gersten, Fuchs, Williams, \& Baker, 2001). Many students with SLD do not use effective strategies to make meaning from text. Ineffective strategy use may result from failure to recall strategies that are needed for comprehension, or it may occur because the student does not implement and monitor strategy use (Bostas \& Padeliadu, 2003).

Another factor that contributes to difficulties with reading comprehension in students with SLD is that they often fail to remember what they read. Several potential causes could contribute individually or collectively to the difficulty in remembering what was read. For example, many students with SLD struggle with reading decoding and reading fluency (Mastropieri \& Scruggs, 2002). As a result, they may exhaust mental energy in trying to read each word accurately, thereby minimizing the opportunity to gain meaning from connected text. Another common observation is that students with SLD show little sensitivity to text structure (Gajria \& Salvia, 1992). Without awareness of how a text is organized, the words on the page appear as a massive block of text, from which the possibility to excavate meaning can be overwhelming. Finally, many students with SLD have low motivation levels for reading (Sideridis, 2005). Limited motivation, in effect, limits engagement with the text. Going through the motions of reading differs from strategically reading for a purpose. In the former case, after reading, it may appear that a reader failed to remember what he or she read when, really, he or she did not 
approach the task with a sense of purpose. Reading comprehension challenges for many students with SLD are compounded by low self-efficacy as a reader (Schiefele, 1996).

\section{Students Learning English as a New Language}

ELs are a heterogeneous group of students from diverse ethnic, racial, cultural, and linguistic backgrounds. Affective factors, background experiences, and proficiency levels in the native language moderate how each student acquires English (Krashen \& Terrell, 1983). Some ELs are dual language learners, or simultaneous bilinguals, who acquire their first language at the same time they are acquiring English. Some ELs are fluent in using interpersonal communication in both their first and second languages but have not developed proficiency with academic vocabulary in either language (Cummins, 1979). Other ELs, sequential bilinguals, are proficient in all language processes of their first language when they begin acquiring English. Due to the variance in language usage, a similar variance in literacy development is observed in second language learners. Ultimately, bilingual literacy develops along a timeline that differs for each student (August \& Shanahan, 2006). The same natural variation is recognized throughout all stages of second language acquisition (Krashen \& Terrell, 1983).

In general, ELs pass through five stages during the process of second language acquisition (Krashen \& Terrell, 1983). The initial phase consists of a preproduction stage, also referred to as the silent period, during which an EL has minimal comprehension of the English language and generally does not verbalize but may nod or point. In fewer than 6 months, an EL will transition into the early production stage. During this second stage, which may last from 6 months to 1 year, an EL will produce 
one- or two-word responses, will begin to use present tense verbs, but will retain limited comprehension of the English language. The third stage, speech emergence, manifests when an EL produces simple sentences, comprehends language, but makes grammar and pronunciation errors. This stage lasts for approximately 1 to 3 years. The fourth stage, intermediate fluency, develops over a 3- to 5-year period, during which the EL demonstrates excellent comprehension while making few grammatical errors. The final stage, advanced fluency, is achieved when the EL has a near-native level of English proficiency. Generally, ELs acquire a near-native level of proficiency over a period that spans or exceeds 5 to 7 years (Krashen \& Terrell, 1983).

Many variables (e.g., cultural factors, personality, motivation levels, and the amount of support provided) affect the length of time it takes a student to acquire nearnative English proficiency (Krashen \& Terrell, 1983). Likewise, a number of factors influence literacy development in second language learners. According to theories of cross-linguistic transfer (Cummins, 1979), certain features of a student's first language will transfer to English. Based on the theory of cross-linguistic transfer, an EL's home language serves as a resource to facilitate literacy acquisition in the second language. Skills, such as making an inference or understanding key ideas in text, transfer broadly across languages (August \& Shanahan, 2006). Other skills, such as production of phonemes, also transfer from the home language to English, at least to the extent that phonemes in the student's first language resemble the approximate 44 phonemes of the English language. For most ELs, however, linguistic transfer is not an automatic occurrence; ELs need explicit strategy instruction to transfer what they know in their 
native language to English (Chomot \& O’Malley, 1996). Even beyond its role in facilitating transfer, to support the complex process of literacy acquisition, explicit instruction is vital to the development of ELs' oral language, vocabulary, and academic language (August \& Shanahan, 2006; Fien, Smith, Baker, Chapparo, Baker, \& Preciado, 2010).

In addition to explicit instruction, effective instruction for ELs incorporates principles of culturally relevant pedagogy (Gay, 2000; Landson-Billings, 1994). The tenets of culturally relevant pedagogy or culturally responsive instruction acknowledge that unconscious cultural perspectives influence teachers' views of the learning process. To become culturally responsive, teachers must first develop awareness of their own cultures and biases (Kalyanpur \& Harry, 2012). Through deliberate examination of one's own cultural beliefs, a teacher can become sensitive to and acquainted with ways in which his or her actions influence others' behaviors (Klingner \& Soltero-Gonzalez, 2009). Moreover, culturally responsive instruction challenges deficit perspectives acknowledging the inherent value in students' background experiences and by helping students connect new learning to their prior knowledge and interests. In contrast, deficit perspectives prevail when students are presumed to lack the capacity—rather than the opportunity_to learn. Within a culturally responsive framework, bilingualism and multiculturalism are perceived as assets to the learning process that ultimately strengthen ELs’ literacy development (August \& Hakuta, 1997). 


\section{Reading Comprehension Difficulties for English Learners}

Difficulties with learning that cannot otherwise be explained are universal; they emerge across languages, cultures, and nations in the world. That is, a student who is learning English as a second language may also have SLD. The two categories (EL and SLD) are not mutually exclusive. At any stage of language proficiency, ELs may struggle to construct meaning from text written in English. Indeed, ELs exhibit more problems with reading comprehension than do native English-speaking, same-age peers (Klingner et al., 2006; Klingner \& Vaughn, 1996). It is important to recognize, however, that these challenges can also stem from cultural or linguistic differences rather than from an underlying disability. There are numerous factors that make reading comprehension difficult for ELs who have not been identified with SLD.

\section{Background Knowledge}

Background knowledge plays a role in influencing reading comprehension. When compared with monolingual, native English-speaking peers, students from culturally and linguistically diverse backgrounds demonstrate variance in their knowledge and experiences relevant to what is read in school (August \& Hakuta, 1997). When a student's background experiences do not correspond with the content of texts encountered in school, he or she is likely to face difficulties in extracting meaning from text. Other contributing factors that may hinder reading comprehension for ELs reside within the experience of having limited proficiency in the English language, such as word reading ability and vocabulary knowledge. 


\section{Word Reading Ability}

Strong word reading ability correlates with strong reading comprehension (August, Francis, Hsu, \& Snow, 2006). If literacy skills are not already developed in the first language or if oral English proficiency is insufficient, reading becomes an abstract and meaningless process (Klingner \& Geisler, 2008). Students who are unfamiliar with alphabetic principle or who are not accustomed to hearing English phonemes will struggle to make sense of the words they are reading.

\section{Vocabulary}

Having an extensive vocabulary correlates with strong comprehension (Cain and Oakhill, 2006). On some measures of comprehension, students perform poorly because of limited vocabulary knowledge (August et al., 2006). ELs may struggle in particular with common words (e.g., pronouns or prepositions), multiple meaning words, and expressions of figurative language (Klingner \& Geisler, 2008). Knowing the meaning of

every word in a text, however, is not always necessary (Gersten et al., 2001). If an EL cannot decode all of the words in a text, or if an EL has no understanding of the underlying concept that some of the words represent, information in the text, when met with nonverbal reasoning skills, can still allow for some amount meaning to be inferred from the text (August et al., 2006).

\section{Word-Calling}

Word-calling is the over-reliance on phonics skills that occurs when word recognition is not yet automatic. When observed to be word-calling, a student's oral reading sounds choppy or lacks fluency. Stanovich (1986) recognized that no empirical 
evidence exists to indicate that decoding a word can happen without extracting some level of meaning. When considering ELs in relation to word-calling, it is important to recognize that unless the words are within the listening comprehension abilities of the child, meaning cannot be extracted from the text. In addition to vocabulary knowledge, motivation and background experiences should be factored into decisions about which texts are appropriate for ELs (Krashen, 2009).

\section{Limited Knowledge of Text Structure}

When an EL's culture differs from the culture experienced in school, he or she may appear to lack the task orientation required to effectively acquire English-reading proficiency. An EL's limited familiarity with discourse features and structures used in text can hinder his or her text comprehension (August et al., 2006). Knowledge of text structure aids a reader in making and confirming predictions while reading, which improves reading comprehension (Gersten et al., 2001). Without explicit instruction on interpreting the text structure, an EL may struggle to offer a retelling, summary, or interpretation that is aligned to authoritative readings of texts.

\section{Affective Factors}

Lack of interest in reading is a likely predictor of poor comprehension (August et al., 2006). Yet, before assuming that an EL lacks interest in reading, it is important to first examine whether texts are meaningful to the student, relevant to his or her experiences, written at an appropriate level, and whether the purposes for reading are clearly communicated to the student. It is also important to consider, when working with ELs, the impact of increased anxiety that may result from the pressures associated with 
adjusting to a new culture or learning a new language (Hoover, 2008). Anxiety and low self-efficacy can undermine reading comprehension (Miranda, Villaescusa, \& VidalAbarca, 1997).

\section{Findings from Major Reviews of Reading Comprehension Intervention Studies}

A total of five meta-analyses and research syntheses on reading comprehension interventions for students with SLD have examined studies published in the past decade, since President Bush signed into law the reauthorization of the IDEA (Dexter \& Hughes, 2011; Edmonds et al., 2009; Kim, Linan-Thompson, \& Misquitta, 2012; Roberts, Torgesen, Boardman, \& Scammacca, 2008; Sencibaugh, 2007). Eight meta-analyses and research syntheses (Berkeley, Scruggs, \& Mastropieri, 2010; Gajria, Jitendra, Sood, \& Sacks, 2007; Gersten et al., 2001; Jitendra, Burgess, \& Gajria, 2011; Kim, Vaughn, Wanzek, \& Wei, 2004; Swanson, 1999; Swanson, Hoskym, \& Lee, 1999; Talbott, Lloyd, \& Tanksersley, 1994) examined studies that were predominantly published prior to December 3, 2004, when the definition of SLD relied solely on identifying a cognitive discrepancy. No major differences emerge when findings of studies that included students who were identified as having SLD through either an ability-achievement discrepancy or through an inadequate response to intervention are compared with findings of studies that included students who were identified as having SLD through only an ability-achievement discrepancy. Moreover, none of the major analyses or syntheses emphasized effectiveness of reading comprehension interventions for culturally or linguistically diverse students with SLD, and none disaggregated findings for ELs with SLD. Based on the limited information reported for ELs with SLD, it cannot be assumed 
that prominent approaches for improving reading comprehension for monolingual students with SLD will be equally effective for ELs with SLD.

Each of the 13 major reviews or meta-analyses reported that effective reading comprehension interventions provided cognitive strategy instruction to make the use of reading comprehension strategies explicit for struggling readers. Means for providing cognitive strategy instruction varied across studies. Most prominently, explicit or direct instruction in single or multiple reading comprehension strategies (e.g., finding the main idea, questioning the author, or making inferences) emerged as an effective practice. Consistently, reading comprehension strategy instruction improved participants' abilities to answer researcher-developed comprehension questions with greater accuracy and to retell what they read with greater precision. In particular, reading comprehension strategy instruction for finding the main idea of expository text was identified as an evidence-based practice (Jitendra et al., 2011). Other cognitive strategies for making the use of reading comprehension strategies explicit involved the use of text enhancements, such as graphic organizers or semantic maps. Findings from two major reviews concurred that use of graphic organizers, structured outlines, or cognitive maps was associated with gains in vocabulary knowledge and inferential knowledge but mainly served to improve factual comprehension (Dexter \& Hughes, 2011; Kim et al., 2004). In each of these studies, participants were trained to fill in graphic organizers that were developed by researchers. For example, participants were given graphic organizers to complete in order to compare and contrast settings of two different texts. None of the participants were trained to develop their own graphic organizers to respond to and 
interpret text. Generalization of the skill of using graphic organizers to comprehend text was not directly assessed. Therefore, outside of the research setting, it cannot be determined whether participants were able to apply the strategy of building cognitive maps or whether using graphic organizers assisted in the comprehension of authentic text encountered across the content areas.

Cognitive strategy instruction has been implemented in a number of different ways. Teacher-mediated instruction, for example, has been implemented through individual instruction (e.g., Malone \& Mastropieri, 1992), small-group instruction (e.g., Jitendra et al., 2000), and whole-class instruction (e.g., Klingner, Vaughn, Arguelles, Hughes, \& Leftwich, 2004). In addition to teacher-mediated instructional methods, peermediated instruction has been found effective for teaching cognitive strategies to students with LD (Sáenz et al., 2005). For example, cooperative grouping and peer-assisted learning strategies have been found effective for students with SLD (Fuchs, Fuchs, Mathes, \& Simmons, 1997; Klingner, Vaughn, \& Schumm, 1998). Regardless of how instruction is mediated, to be effective, strong modeling and consistent monitoring of strategies are necessary for ensuring mastery (Gersten et al., 2001). The most robust gains that were identifiable on standardized measures of reading comprehension achievement were reported by researchers who favored peer-mediated instruction or mixed-ability grouping to deliver cognitive strategy instruction (Sáenz et al., 2005).

\section{Prominent Approaches to Improving Reading Comprehension}

In a landmark study, Durkin (1979) called attention to the need for change in reading comprehension instruction. Durkin's observations of classroom reading 
instruction revealed that typical reading instruction involved three practices: mentioning, practicing, and assessing. Teachers mentioned the skills that students were expected to use. Students completed worksheets to practice the skills, most of which focused on surface-level or literal comprehension. Then, teachers assessed whether students used the skills or not. In nearly 4,000 min of observations in fourth-grade classrooms, reading comprehension instruction did not occur. Lack of engagement and lack of improvements in reading comprehension were revealed in connection with the observed lack of reading comprehension instruction. Durkin's observations significantly influenced research in reading comprehension (Dole, Duffy, Roehler, \& Pearson, 1991).

Over the past 35 years, many of the instructional practices used to improve reading comprehension for struggling readers with SLD derived from observations, reports, and studies that uncovered how good readers understand and learn from text (Klingner, Vaughn, \& Boardman, 2007). Good readers think strategically about text while they read (Paris, Wasik, \& Turner, 1991). Research conducted over the past three and a half decades has investigated metacognitive strategies that readers use to monitor for meaning. This has resulted in a number of intervention studies on the use of cognitive strategies to make explicit reading comprehension strategies (e.g., questioning, summarizing, predicting). The premise behind these interventions is that students who struggle in reading comprehension do so because they are not able to effectively and efficiently use metacognitive strategies.

A number of cognitive tasks must be engaged to enact and construct meaning from text (Tablott et al., 1994). For example, readers must recognize words and make 
connections between words, familiar concepts, and meanings. Also, readers must construct a mental representation of the text by interpreting sentences and paragraphs (Perfetti, 1985). Finally, readers must use strategies to remember and interpret text (Ryan, 1981). A number of characteristics associated with SLD (e.g., difficulties with decoding, difficulties with sustaining attention, or difficulties with working memory) can impede the meaning-making process. One prominent approach to reading comprehension instruction addresses these concerns for students with SLD by focusing on explicitly teaching cognitive strategies.

A cognitive strategy, according to Rosenshine (1995) is "a heuristic or guide that serves to support or facilitate the learner as he or she develops the internal procedures that enable them [sic] to perform higher level operations" (p. 266). Successful reading comprehension hinges on the activation of reading strategies. After examining hundreds of studies, distinctions between reading strategies, cognitive strategies, and comprehension monitoring strategies could be found easily (Yang, 2006). Cheng (1998) illuminated a possible distinction by offering an explanation that strategic readers know which strategies to use and also are aware of when, why, and how to use the strategies. That is, reading strategies generally consist of techniques readers can use to find the main idea, summarize, paraphrase, visualize, question, infer, and use schema. Reading strategies are propelled by cognitive strategies, which may include, among others, metacognition (i.e., thinking about thinking) and self-monitoring (i.e., being aware of what one knows and does not know). 
The literature on reading comprehension interventions for students with SLD reflects various models for cognitive strategy instruction, all of which make explicit the use of reading comprehension strategies while reading narrative and expository textual information. For example, one model focuses on teaching paraphrasing, a reading comprehension strategy. In this model, students use an acronym (RAP) as a cue to remember the steps of the strategy: (a) read a paragraph, (b) ask yourself questions, and (c) put the main idea in your own words (Schumaker, Denton, \& Deschler, 1984). The model intends for students to practice learning how and why to use the strategy while reading a variety of informational texts. Then, as students become more familiar with how to use the strategy, they learn when to apply it (Berry, Hall, \& Gildroy, 2004).

In five group design studies, researchers have provided evidence of the effectiveness of providing instruction in a single strategy (e.g., summarizing, questioning, or finding the main idea) or in multiple strategies (e.g., reciprocal teaching, which combines predicting, questioning, and summarizing) for improving text comprehension (Gajria et al., 2007; Gersten et al., 2001; Mastropieri, Scruggs, Bakken, \& Whedon, 1996; Sencibaugh, 2007; Swanson, 1999). One common feature among studies on reading comprehension strategy instruction is the use of direct or explicit instruction. Whereas Durkin (1979) observed a mention-practice-assess procedure for teaching comprehension skills, explicit instruction uses a procedure that systematically models or demonstrates the use of strategy, offers guided practice with feedback, and gradually releases responsibility for independent use of the strategy to the students. 
Although moderate to strong effect sizes were associated with gains in reading comprehension achievement immediately following an intervention across many studies that used direct or explicit instruction to teach comprehension strategies, one criticism relates to diminishing effects after withdrawal of the intervention (Gajria et al., 2007; Wanzek et al., 2013). Within the corpus of 19 studies on explicit reading comprehension strategy instruction included in major reviews, the majority assessed intervention effects through comparing posttest scores with pretest scores. Only five studies assessed maintenance 1 to 16 weeks after the intervention was withdrawn; diminishing effects were reported in each of the studies (Graves, 1986; Graves \& Levin, 1989; Jitendra, Cole, Hoppes, \& Wilson, 1998; Jitendra, Hoppes, \& Xin, 2000; Kim, Vaughn, Klingner, Woodruff, Reutebuch, \& Kouzekanani, 2006).

Another prominent approach to reading comprehension instruction holds the potential to address concerns about participants' internalization of explicitly taught comprehension strategies by ensuring that participants take ownership of their learning through using self-regulation procedures. Using a self-regulated strategy design (SRSD), teaching students how to think is just as important as teaching students what to think (Harris, 1982). This approach combines explicit instruction in using comprehension strategies with instruction in using self-regulation procedures (Harris \& Graham, 1999). Procedures for self-regulation include: self-instruction, goal setting, self-monitoring, and self-reinforcement. An important step in SRSD instruction is that students memorize the strategy steps (Mason, 2013). This step is pivotal to ensuring maintenance and generalization of the effects of strategy instruction. Findings from four empirical studies 
confirm positive performance effects following SRSD instruction for teaching struggling readers to think before, while, and after reading (Hedin, Mason, \& Gaffney, 2011; Mason, Snyder, Sukhram, \& Kedem, 2006; Rogevich \& Perin, 2008).

\section{Reading Comprehension Interventions for English Learners with Specific Learning Disabilities}

The research base on effective reading comprehension interventions for ELs who struggle with reading comprehension or who have SLD is limited to 20 studies (see Table 2). Of these 20 studies, researchers in only four studies have demonstrated, through teacher-mediated instruction (Bos \& Anders, 1992; Jiménez, 1997) or through peermeditated instruction (Klingner \& Vaughn, 1996; Sáenz, et al., 2005) how the language development needs of ELs with SLD can be supported while teaching reading comprehension strategies in order to improve participants' reading comprehension achievement.

Examining the outcomes relevant to reading comprehension achievement requires simultaneous attention to methodological rigor. Of the 20 reading comprehension studies that have been conducted over the past 20 years with ELs who struggle in reading, fewer than 10 studies satisfied quality indicators (QIs) as specified by the Council for Exceptional Children (CEC, 2014). The QIs were established to ensure that studies have minimal methodological features to merit confidence in their findings. The QIs address eight specific domains: context and setting, participant characteristics, the intervention agent, description of the practice or intervention, implementation fidelity, internal validity, outcome measures, and data analysis. 
Consistently, the 20 identified studies on reading comprehension interventions did not provide sufficient information related to dosage fidelity. Some of the studies that used a single-subject research design did not consistently control for threats to internal validity, such as history, maturation, or attrition (Montoya, 2008; Santoro et al., 2006). Additionally, some studies that used an experimental design did not provide an explanation of procedures that would limit the control group's access to the intervention (Bos \& Anders, 1992; Klingner \& Vaughn, 1996). Five of the studies included fewer than three ELs with SLD, which, therefore, limits any knowledge claims that can be made about the effectiveness of the interventions for ELs with SLD (Denton, Anthony, Parker, \& Hasbrouck, 2004; Santoro, Jitendra, Starosta, \& Stacks, 2006; Vaughn, LinanThompson, et al., 2006; Vaughn, Mathes, \& Linan-Thompson, 2006; Wanzek \& Roberts, 2012). Two studies included a sufficient number of ELs with SLD to warrant confidence in their findings (i.e., more than three participants for single-subject research and more than 40 for experimental group design research) but have not been replicated in other settings with other participants (Helman et al., 2014; Sáenz, et al., 2005). Given the small number of participants and the lack of replication, none of the reading comprehension interventions reported among the 20 studies in the research base have met criteria for evidence-based classification.

In addition to concerns about the knowledge claims that can be made from a research base that lacks methodological rigor or that has not yet met minimum criteria for evidence-based classification, adherence to tenets of culturally responsive research is a concern. To create a means for analyzing the extent to which intervention research, from 
its design to its implementation and dissemination, is culturally responsive, Trainor and Bal (2014) developed a rubric for culturally responsive research. This rubric examines cultural responsiveness in 15 categories, which include: foundational constructs of the study, relevancy of the research problem, critical and comprehensive review of the relevant literature, justification of the theoretical framework, description of participants, description of researchers and interventionists, description of sampling procedures, description of the research setting, description of data collection strategies, ecology of the intervention, intervention design, assessment of intervention efficacy, presentation of findings, analysis and interpretation, and discussion of dissemination.

Of the 20 known reading comprehension intervention studies that were conducted between January 1, 1992 and May 5, 2015, only one study satisfied criteria to reflect cultural responsiveness (Jiménez, 1997). The ecology of the cognitive strategy instruction that Jiménez provided in his qualitative study in a bilingual special education classroom was aligned with participants' experiences and preferences; moreover, throughout the study participants' cultural and personal identities were affirmed through the use of individualized schema-building activities and the integration of culturally relevant text. None of the 19 studies that used an experimental research design satisfied criteria for cultural responsiveness, as outlined by Trainor and Bal (2014). In a majority of the experimental studies, authors described the interventionist by providing a title (e.g., teacher, graduate assistant) and by explaining credentials (e.g., having a teaching license) but did not describe enough demographic background information to determine relational positions between participants and interventionists (e.g., membership to participants' 
cultural group, proficiency levels with participants' native language, power status). Most researchers did not describe the design of the intervention in a way that could reveal whether the intervention would affirm participants' cultural and personal identities or "facilitate development of participants' awareness and capacity to challenge inequities that they experience" (Trainor \& Bal, 2014, p 208).

Due to limitations with cultural responsiveness and with the methodological rigor of the existing research base, there remains a need to develop and test rigorously the effectiveness of reading comprehension interventions for ELs with SLD. Moreover, given the federally mandated emphasis on using scientifically based instruction and the negative performance and achievement outcomes experienced by students who have disabilities and who are not fully proficient in English, minimizing this gap in the literature is an urgent priority. 
Table 2

Key Information from Reading Comprehension Intervention Studies

\begin{tabular}{|c|c|c|c|c|c|c|}
\hline Study & Participants & Purpose & $\begin{array}{l}\text { Independent } \\
\text { Variable }\end{array}$ & $\begin{array}{l}\text { Dependent } \\
\text { Variable }\end{array}$ & Results & Findings \\
\hline \multicolumn{7}{|c|}{ Reading Comprehension Strategies } \\
\hline $\begin{array}{l}\text { Bos \& } \\
\text { Anders, } \\
1992\end{array}$ & $\begin{array}{l}103 \text { ELs } \\
\text { with } \\
\text { learning } \\
\text { disabilities } \\
\text { with Spanish } \\
\text { as L1 in } \\
\text { upper } \\
\text { elementary } \\
\text { and middle } \\
\text { school } \\
\text { classrooms } \\
\text { during } \\
\text { science or } \\
\text { social } \\
\text { studies } \\
\text { instruction }\end{array}$ & $\begin{array}{l}\text { To } \\
\text { examine } \\
\text { effects of } \\
\text { interactive } \\
\text { teaching } \\
\text { strategies } \\
\text { on reading } \\
\text { compre- } \\
\text { hension } \\
\text { using a } \\
\text { pre- post } \\
\text { exper- } \\
\text { imental } \\
\text { design }\end{array}$ & $\begin{array}{l}\text { Researchers } \\
\text { and special } \\
\text { education } \\
\text { teachers } \\
\text { delivered } \\
\text { instruction } \\
\text { in Spanish } \\
\text { and English } \\
50 \text { min per } \\
\text { day over a } \\
12 \text {-week } \\
\text { period; } \\
\text { comparison } \\
\text { group } \\
\text { received } \\
\text { traditional } \\
\text { instruction. }\end{array}$ & $\begin{array}{l}\text { Scores on } \\
\text { researcher- } \\
\text { developed } \\
\text { multiple } \\
\text { choice } \\
\text { vocabulary } \\
\text { and } \\
\text { compre- } \\
\text { hension } \\
\text { tests }\end{array}$ & $\begin{array}{l}\text { Scores } \\
\text { increased } \\
\text { from } \\
\text { pretest to } \\
\text { posttest; } \\
\text { gains were } \\
\text { similar to } \\
\text { those of } \\
\text { same age } \\
\text { average } \\
\text { peers. }\end{array}$ & $\begin{array}{l}\text { Providing } \\
\text { explicit } \\
\text { instruction in } \\
\text { using } \\
\text { strategies to } \\
\text { extract } \\
\text { meaning from } \\
\text { content area } \\
\text { texts with } \\
\text { collaborative } \\
\text { problem } \\
\text { solving created } \\
\text { a motivating } \\
\text { structure for } \\
\text { learning. }\end{array}$ \\
\hline $\begin{array}{l}\text { Halter- } \\
\text { man, } \\
2013\end{array}$ & $\begin{array}{l}19 \text { students } \\
\text { with SLD; } \\
11 \text { ELs (10 } \\
\text { used } \\
\text { Spanish as } \\
\text { L1) in two } \\
\text { high school } \\
\text { special } \\
\text { education } \\
\text { classes in } \\
\text { San } \\
\text { Francisco }\end{array}$ & $\begin{array}{l}\text { To } \\
\text { examine } \\
\text { effects of } \\
\text { the RAP } \\
\text { and } \\
\text { semantic } \\
\text { mapping } \\
\text { strategies } \\
\text { on compre- } \\
\text { hension } \\
\text { using a } \\
\text { quasi- } \\
\text { experiment } \\
\text { al design }\end{array}$ & $\begin{array}{l}\text { English- } \\
\text { only } \\
\text { instruction } \\
\text { in RAP } \\
\text { and a } \\
\text { semantic } \\
\text { mapping } \\
\text { strategy as } \\
\text { compared } \\
\text { with } \\
\text { traditional } \\
\text { instruction } \\
\text { for } 540 \\
\text { min across } \\
9 \text { days }\end{array}$ & $\begin{array}{l}\text { Results from } \\
\text { an oral } \\
\text { language } \\
\text { proficiency } \\
\text { test and } \\
\text { reading } \\
\text { compre- } \\
\text { hension } \\
\text { scores on } \\
\text { the Gates } \\
\text { Mac- Ginitie } \\
\text { Reading } \\
\text { Test }\end{array}$ & $\begin{array}{l}\text { ELs } \\
\text { recalled } \\
\text { steps of the } \\
\text { RAP } \\
\text { strategy; } \\
\text { non-ELs } \\
\text { recalled } \\
\text { more steps } \\
\text { of the } \\
\text { semantic- } \\
\text { mapping } \\
\text { strategy. }\end{array}$ & $\begin{array}{l}\text { RAP holds } \\
\text { promise; } \\
\text { more } \\
\text { research is } \\
\text { needed to } \\
\text { determine } \\
\text { maintenance } \\
\text { effects. }\end{array}$ \\
\hline
\end{tabular}

(Table Continues) 


\begin{tabular}{|c|c|c|c|c|c|c|}
\hline Study & Participants & Purpose & $\begin{array}{l}\text { Independent } \\
\text { Variable }\end{array}$ & $\begin{array}{l}\text { Dependent } \\
\text { Variable }\end{array}$ & Results & Findings \\
\hline $\begin{array}{l}\text { Jiménez } \\
\text {, } 1997\end{array}$ & $\begin{array}{l}5 \text { Latino/a } \\
\text { seventh- } \\
\text { grade } \\
\text { students } \\
\text { whose L1 } \\
\text { was } \\
\text { Spanish } \\
\text { and who } \\
\text { had low } \\
\text { levels of } \\
\text { literacy in } \\
\text { English } \\
\text { during } \\
\text { individual } \\
\text { and small- } \\
\text { group } \\
\text { instruction }\end{array}$ & $\begin{array}{l}\text { To } \\
\text { investigate } \\
\text { strategic } \\
\text { literacy } \\
\text { knowledge } \\
\text { of five low- } \\
\text { literacy } \\
\text { Latinos in } \\
\text { middle } \\
\text { school } \\
\text { using a } \\
\text { qualitative } \\
\text { design. } \\
\text { Also, to } \\
\text { evaluate } \\
\text { effects of a } \\
\text { formative } \\
\text { experiment } \\
\text { that } \\
\text { provided } \\
\text { cognitive } \\
\text { strategy } \\
\text { lessons. }\end{array}$ & $\begin{array}{l}\text { Cognitive } \\
\text { strategy } \\
\text { instruction } \\
\text { provided in } \\
\text { Spanish and } \\
\text { English to } \\
\text { target } \\
\text { questioning, } \\
\text { making } \\
\text { inferences, } \\
\text { and } \\
\text { determining } \\
\text { meaning of } \\
\text { unknown } \\
\text { words }\end{array}$ & $\begin{array}{l}\text { Observed } \\
\text { literacy } \\
\text { behaviors }\end{array}$ & $\begin{array}{l}\text { Training } \\
\text { in } \\
\text { cognitive } \\
\text { strategies } \\
\text { guided } \\
\text { students } \\
\text { to } \\
\text { verbally } \\
\text { identify } \\
\text { that } \\
\text { "reading } \\
\text { is } \\
\text { thinking" } \\
\text { and } \\
\text { allowed } \\
\text { them to } \\
\text { apply } \\
\text { strategies } \\
\text { and } \\
\text { "think } \\
\text { aloud" } \\
\text { while } \\
\text { making } \\
\text { meaning } \\
\text { from text }\end{array}$ & $\begin{array}{l}\text { Compre- } \\
\text { hension } \\
\text { instruction } \\
\text { should } \\
\text { explicitly } \\
\text { teach } \\
\text { cognitive } \\
\text { strategies for } \\
\text { compre- } \\
\text { hension (e.g., } \\
\text { use of } \\
\text { cognates, } \\
\text { translating, } \\
\text { and transfer } \\
\text { from L1 to } \\
\text { L2) while } \\
\text { using } \\
\text { culturally } \\
\text { relevant and } \\
\text { familiar text. }\end{array}$ \\
\hline $\begin{array}{l}\text { Kling- } \\
\text { ner \& } \\
\text { Vau- } \\
\text { ghn, } \\
1996\end{array}$ & $\begin{array}{l}26 \text { ELs with } \\
\text { SLD whose } \\
\text { L1 was } \\
\text { Spanish in } \\
\text { special } \\
\text { education } \\
\text { classes in an } \\
\text { urban middle } \\
\text { school }\end{array}$ & $\begin{array}{l}\text { To } \\
\text { investigate } \\
\text { effects of } \\
\text { two } \\
\text { approaches } \\
\text { for } \\
\text { providing } \\
\text { reading } \\
\text { compre- } \\
\text { hension } \\
\text { strategy } \\
\text { instruction } \\
\text { using pre- } \\
\text { post } \\
\text { experiment } \\
\text {-al design }\end{array}$ & $\begin{array}{l}27 \text { days of } \\
\text { instruction } \\
\text { that included } \\
\text { reciprocal } \\
\text { teaching } \\
\text { with cross- } \\
\text { age tutoring } \\
\text { or reciprocal } \\
\text { teaching } \\
\text { with } \\
\text { cooperative } \\
\text { grouping }\end{array}$ & $\begin{array}{l}\text { Scores on } \\
\text { measures } \\
\text { of } \\
\text { comprehe } \\
\text { nsion } \\
\text { (Woodcoc } \\
\text { k Johnson } \\
\text { III and } \\
\text { Gates } \\
\text { Mac- } \\
\text { Ginitie) }\end{array}$ & $\begin{array}{l}\text { Both } \\
\text { groups } \\
\text { made } \\
\text { progress } \\
\text { but there } \\
\text { were no } \\
\text { significa } \\
\text { nt differ- } \\
\text { ences } \\
\text { between } \\
\text { groups }\end{array}$ & $\begin{array}{l}\text { Students } \\
\text { with low } \\
\text { decoding } \\
\text { skills } \\
\text { made } \\
\text { gains. } \\
\text { Cross-age } \\
\text { tutoring } \\
\text { led to high } \\
\text { task } \\
\text { engage- } \\
\text { ment. Use } \\
\text { of L1 } \\
\text { supported } \\
\text { compre- } \\
\text { hension. }\end{array}$ \\
\hline
\end{tabular}

(Table Continues) 


\begin{tabular}{|c|c|c|c|c|c|c|}
\hline Study & Participants & Purpose & $\begin{array}{l}\text { Independent } \\
\text { Variable }\end{array}$ & $\begin{array}{l}\text { Dependent } \\
\text { Variable }\end{array}$ & Results & Findings \\
\hline $\begin{array}{l}\text { Mc-Elvain, } \\
2010\end{array}$ & $\begin{array}{l}75 \text { fourth- } \\
\text { to sixth- } \\
\text { grade ELs } \\
\text { at an } \\
\text { intermed- } \\
\text { iate level of } \\
\text { English } \\
\text { language } \\
\text { proficiency } \\
\text { (L1 not } \\
\text { provided) } \\
\text { in two low- } \\
\text { income } \\
\text { elementary } \\
\text { schools in } \\
\text { northern } \\
\text { California }\end{array}$ & $\begin{array}{l}\text { To } \\
\text { examine } \\
\text { academic } \\
\text { and } \\
\text { psycho- } \\
\text { social } \\
\text { effects of } \\
\text { trans- } \\
\text { actional } \\
\text { literacy } \\
\text { circles on } \\
\text { reading } \\
\text { compre- } \\
\text { hension of } \\
\text { at risk ELs } \\
\text { using a } \\
\text { pre- post } \\
\text { experi- } \\
\text { mental } \\
\text { design. }\end{array}$ & $\begin{array}{l}7 \text { months of } \\
\text { transactiona } \\
1 \text { literacy } \\
\text { circles } \\
\text { program }\end{array}$ & $\begin{array}{l}\text { Scores on the } \\
\text { California } \\
\text { STAR Test } \\
\text { and the } \\
\text { California } \\
\text { Achievement } \\
\text { Test -6; } \\
\text { qualitative } \\
\text { reading } \\
\text { inventory } \\
\text { results }\end{array}$ & $\begin{array}{l}\text { No } \\
\text { significant } \\
\text { difference } \\
\text { in } \\
\text { perform- } \\
\text { ance } \\
\text { between } \\
\text { treatment } \\
\text { and } \\
\text { control } \\
\text { groups. }\end{array}$ & $\begin{array}{l}\text { Collabor } \\
\text {-ative } \\
\text { conversa } \\
\text {-tions } \\
\text { about } \\
\text { relevant, } \\
\text { multicult } \\
\text {-ural text } \\
\text { made an } \\
\text { impact } \\
\text { on } \\
\text { psycho- } \\
\text { social } \\
\text { factors, } \\
\text { per } \\
\text { observa- } \\
\text { tion and } \\
\text { inter- } \\
\text { view } \\
\text { data. }\end{array}$ \\
\hline $\begin{array}{l}\text { Sáenz } \\
\text { et al., } \\
2005\end{array}$ & $\begin{array}{l}119 \text { ELs } \\
\text { with } \\
\text { learning } \\
\text { disabilities } \\
\text { whose L1 } \\
\text { was } \\
\text { Spanish in } \\
\text { third to } \\
\text { sixth grade } \\
\text { classrooms } \\
\text { in south } \\
\text { Texas }\end{array}$ & $\begin{array}{l}\text { To assess } \\
\text { effects of } \\
\text { reciprocal } \\
\text { class-wide } \\
\text { peer- } \\
\text { tutoring } \\
\text { strategy } \\
\text { on reading } \\
\text { perform- } \\
\text { ance of } \\
\text { Spanish- } \\
\text { speaking } \\
\text { students } \\
\text { using a } \\
\text { pre- post } \\
\text { experi- } \\
\text { mental } \\
\text { design. }\end{array}$ & $\begin{array}{l}\text { Peer- } \\
\text { Assisted } \\
\text { Learning } \\
\text { Strategy } \\
\text { (PALS) } \\
\text { instruction } \\
\text { three times } \\
\text { per week } \\
\text { for } 35 \text { min } \\
\text { over } 15 \\
\text { weeks, } \\
\text { allowing } \\
\text { use of L1 } \\
\text { and L2 }\end{array}$ & $\begin{array}{l}\text { Scores on the } \\
\text { Compre- } \\
\text { hensive } \\
\text { Reading } \\
\text { Assessment } \\
\text { Battery } \\
\text { (CRAB) }\end{array}$ & $\begin{array}{l}\text { Strong } \\
\text { results } \\
\text { from pre- } \\
\text { test to } \\
\text { posttest; } \\
\text { ES }=.86 \\
\text { for low } \\
\text { achieving } \\
\text { ELs }\end{array}$ & $\begin{array}{l}\text { Strong } \\
\text { results } \\
\text { occurred } \\
\text { but a } \\
\text { high } \\
\text { level of } \\
\text { technical } \\
\text { assis- } \\
\text { tance } \\
\text { was } \\
\text { provided }\end{array}$ \\
\hline
\end{tabular}

(Table Continues) 


\begin{tabular}{|c|c|c|c|c|c|c|}
\hline Study & Participants & Purpose & $\begin{array}{l}\text { Independent } \\
\text { Variable }\end{array}$ & $\begin{array}{l}\text { Dependent } \\
\text { Variable }\end{array}$ & Results & Findings \\
\hline \multicolumn{7}{|c|}{ Vocabulary and Reading Comprehension } \\
\hline $\begin{array}{l}\text { Albers } \\
\& \text { Hoff- } \\
\text { man, } \\
2012\end{array}$ & $\begin{array}{l}3 \text { ELs in } \\
\text { third } \\
\text { grade. All } \\
\text { were at } \\
\text { the } \\
\text { intermedia } \\
\text { te level of } \\
\text { English } \\
\text { proficien- } \\
\text { cy and } \\
\text { were } \\
\text { identified } \\
\text { as } \\
\text { struggling } \\
\text { readers; } \\
\text { all used } \\
\text { Spanish as } \\
\text { L1 in a } \\
\text { Mid- } \\
\text { western } \\
\text { element- } \\
\text { ary school } \\
\text { where 1:1 } \\
\text { instruction } \\
\text { was } \\
\text { provided } \\
\text { in a } \\
\text { separate } \\
\text { setting }\end{array}$ & $\begin{array}{l}\text { To examine } \\
\text { effects of an } \\
\text { English only } \\
\text { folding-in } \\
\text { technique } \\
\text { combined } \\
\text { with self- } \\
\text { graphing } \\
\text { procedures } \\
\text { on } \\
\text { vocabulary } \\
\text { sight word } \\
\text { recognition } \\
\text { using a } \\
\text { multiple } \\
\text { baseline } \\
\text { across } \\
\text { participants; } \\
\text { to determine } \\
\text { if increases } \\
\text { in sight } \\
\text { word } \\
\text { vocabulary } \\
\text { are } \\
\text { associated } \\
\text { with } \\
\text { increases in } \\
\text { fluency and } \\
\text { compre- } \\
\text { hension }\end{array}$ & $\begin{array}{l}\text { Interspers- } \\
\text { ing new } \\
\text { words with } \\
\text { known } \\
\text { words on a } \\
\text { flash card } \\
\text { drill } \\
\text { technique } \\
\text { that included } \\
\text { self- } \\
\text { graphing } \\
\text { procedures; } \\
\text { three times } \\
\text { per week for } \\
7 \text { weeks, } 15 \\
\text { to } 20 \text { min } \\
\text { per session }\end{array}$ & $\begin{array}{l}\text { Performance } \\
\text { on Reading } \\
\text { Curriculum- } \\
\text { Based } \\
\text { Measures in } \\
\text { oral reading } \\
\text { fluency, } \\
\text { compre- } \\
\text { hension } \\
\text { (maze tasks), } \\
\text { and the } \\
\text { percentage of } \\
\text { originally } \\
\text { unknown } \\
\text { words that } \\
\text { were } \\
\text { correctly } \\
\text { identified } \\
\text { after the } \\
\text { intervention }\end{array}$ & $\begin{array}{l}\text { Each } \\
\text { particip- } \\
\text { ant } \\
\text { increased } \\
\text { scores on } \\
\text { fluency } \\
\text { and maze } \\
\text { tasks } \\
\text { with } \\
100 \% \\
\text { PND. } \\
\text { Each } \\
\text { particip- } \\
\text { ant } \\
\text { increased } \\
\text { percent- } \\
\text { age of } \\
\text { known } \\
\text { words. }\end{array}$ & $\begin{array}{l}\text { Compon- } \\
\text { ent } \\
\text { analysis is } \\
\text { needed to } \\
\text { determine } \\
\text { effects of } \\
\text { the goal- } \\
\text { setting } \\
\text { procedure } \\
\text { and the } \\
\text { folding in } \\
\text { technique. } \\
\text { Future } \\
\text { replication } \\
\text { of this } \\
\text { study } \\
\text { should } \\
\text { context- } \\
\text { ualize } \\
\text { inform- } \\
\text { ation } \\
\text { about } \\
\text { perform- } \\
\text { ance } \\
\text { relative to } \\
\text { same-age } \\
\text { peers } \\
\text { before and } \\
\text { after } \\
\text { interven- } \\
\text { tion. }\end{array}$ \\
\hline
\end{tabular}

(Table Continues) 


\begin{tabular}{|c|c|c|c|c|c|c|}
\hline Study & Participants & Purpose & $\begin{array}{l}\text { Independent } \\
\text { Variable }\end{array}$ & $\begin{array}{l}\text { Dependent } \\
\text { Variable }\end{array}$ & Results & Findings \\
\hline $\begin{array}{l}\text { Helman, } \\
\text { et al., } \\
2014\end{array}$ & $\begin{array}{l}3 \text { ELs at an } \\
\text { inter- } \\
\text { mediate } \\
\text { proficiency } \\
\text { level; all } \\
\text { with SLD; } \\
\text { all with } \\
\text { Spanish as } \\
\text { L1 in an } \\
\text { Urban high } \\
\text { school in } \\
\text { Eastern } \\
\text { United } \\
\text { States; } 30- \\
45 \text { min } \\
\text { sessions for } \\
135 \text { to } 270 \\
\text { min total } \\
\text { delivered } \\
1: 1 \text { in a } \\
\text { separate } \\
\text { setting }\end{array}$ & $\begin{array}{l}\text { To evaluate } \\
\text { effects of } \\
\text { the Clue } \\
\text { Word } \\
\text { Strategy on } \\
\text { acquisition } \\
\text { of science } \\
\text { vocabulary } \\
\text { and to } \\
\text { examine } \\
\text { whether } \\
\text { increased } \\
\text { word } \\
\text { knowledge } \\
\text { led to } \\
\text { improved } \\
\text { reading } \\
\text { comprehen } \\
\text { sion } \\
\text { through a } \\
\text { multiple } \\
\text { baseline } \\
\text { across } \\
\text { participants }\end{array}$ & $\begin{array}{l}135 \text { to } 270 \\
\text { min of } \\
\text { training in } \\
\text { the clue } \\
\text { word } \\
\text { strategy } \\
\text { which } \\
\text { combines } \\
\text { morphologic } \\
\text { al and } \\
\text { contextual } \\
\text { analysis to } \\
\text { aid in - } \\
\text { vocabulary } \\
\text { development }\end{array}$ & $\begin{array}{l}\text { Scores on a } \\
\text { test of } \\
\text { reading } \\
\text { comprehensio } \\
\text { n (TORC-4), } \\
\text { scores on a } \\
\text { word } \\
\text { knowledge } \\
\text { test, a } \\
\text { morpheme } \\
\text { test, and a test } \\
\text { of strategy } \\
\text { use and } \\
\text { strategy } \\
\text { knowledge }\end{array}$ & $\begin{array}{l}\text { Two partic- } \\
\text { ipants } \\
\text { made gains } \\
\text { in compre- } \\
\text { hension } \\
\text { from pre to } \\
\text { posttest; all } \\
\text { improved } \\
\text { in strategy } \\
\text { knowledge, } \\
\text { strategy } \\
\text { use, word } \\
\text { know- } \\
\text { ledge, and } \\
\text { morpheme } \\
\text { knowledge }\end{array}$ & $\begin{array}{l}\text { Explicit } \\
\text { strategy } \\
\text { instruction } \\
\text {, scaffolds } \\
\text { during } \\
\text { instruction } \\
\text {, multiple } \\
\text { opportunit } \\
\text {-ies for } \\
\text { practice, } \\
\text { cognitive } \\
\text { strategy } \\
\text { instruction } \\
\text {, and use } \\
\text { of graphic } \\
\text { organizers } \\
\text { are } \\
\text { important } \\
\text { to } \\
\text { integrate } \\
\text { into an } \\
\text { interven- } \\
\text { tion to } \\
\text { support } \\
\text { English } \\
\text { language } \\
\text { acquisi- } \\
\text { tion }\end{array}$ \\
\hline
\end{tabular}

(Table Continues) 


\begin{tabular}{|c|c|c|c|c|c|c|}
\hline Study & Participants & Purpose & $\begin{array}{l}\text { Independent } \\
\text { Variable }\end{array}$ & $\begin{array}{l}\text { Dependent } \\
\text { Variable }\end{array}$ & Results & Findings \\
\hline $\begin{array}{l}\text { Proctor } \\
\text { et al., } \\
2007\end{array}$ & $\begin{array}{l}30 \text { fourth- } \\
\text { grade } \\
\text { struggling } \\
\text { readers, } \\
\text { including } 16 \\
\text { ELs with } \\
\text { Spanish as } \\
\text { their L1 in a } \\
\text { computer } \\
\text { lab in a Title } \\
1 \text { school in } \\
\text { an affluent } \\
\text { area in } \\
\text { southern } \\
\text { California }\end{array}$ & $\begin{array}{l}\text { To examine } \\
\text { effects of } \\
\text { universally } \\
\text { designed } \\
\text { (English } \\
\text { only) } \\
\text { digital texts } \\
\text { with } \\
\text { embedded } \\
\text { supports } \\
\text { for } \\
\text { vocabulary } \\
\text { and } \\
\text { compre- } \\
\text { hension in } \\
\text { an } \\
\text { exploratory } \\
\text { study }\end{array}$ & $\begin{array}{l}5 \text { weeks of } \\
\text { instruction } \\
\text { using eight } \\
\text { hypertexts } \\
\text { with } \\
\text { embedded } \\
\text { supports, } \\
\text { which } \\
\text { included } \\
\text { drama- } \\
\text { tization of } \\
\text { vocabulary } \\
\text { words and } \\
\text { digital } \\
\text { coaching } \\
\text { avatars that } \\
\text { modeled and } \\
\text { prompted } \\
\text { use of } \\
\text { compre- } \\
\text { hension } \\
\text { strategies }\end{array}$ & $\begin{array}{l}\text { Scores on } \\
\text { measures of } \\
\text { vocabulary } \\
\text { and compre- } \\
\text { hension } \\
\text { (Gates Mac } \\
\text { Ginitie) and } \\
\text { results from } \\
\text { an event } \\
\text { usage tracker } \\
\text { to log } \\
\text { frequency } \\
\text { with which } \\
\text { embedded } \\
\text { supports were } \\
\text { activated }\end{array}$ & $\begin{array}{l}\text { Gains } \\
\text { were } \\
\text { reported } \\
\text { on all } \\
\text { measures } \\
\text { from pre } \\
\text { to } \\
\text { posttest; } \\
\text { gains for } \\
\text { ELs } \\
\text { exceeded } \\
\text { gains for } \\
\text { mono- } \\
\text { lingual } \\
\text { English } \\
\text { speakers }\end{array}$ & $\begin{array}{l}\text { Universally } \\
\text { designed } \\
\text { digital texts } \\
\text { offer support } \\
\text { that can aid } \\
\text { in compre- } \\
\text { hension and } \\
\text { increase } \\
\text { word } \\
\text { knowledge; } \\
\text { future } \\
\text { research } \\
\text { should } \\
\text { examine } \\
\text { whether } \\
\text { traditional } \\
\text { compre- } \\
\text { hension } \\
\text { strategies are } \\
\text { effective for } \\
\text { promoting } \\
\text { compre- } \\
\text { hension of } \\
\text { web-based } \\
\text { text. }\end{array}$ \\
\hline
\end{tabular}

(Table Continues) 


\begin{tabular}{|c|c|c|c|c|c|c|}
\hline Study & Participants & Purpose & $\begin{array}{l}\text { Independent } \\
\text { Variable }\end{array}$ & $\begin{array}{l}\text { Dependent } \\
\text { Variable }\end{array}$ & Results & Findings \\
\hline \multicolumn{7}{|c|}{ Fluency, Vocabulary, and Comprehension } \\
\hline $\begin{array}{l}\text { Landa, } \\
2009\end{array}$ & $\begin{array}{l}4 \text { ELs with } \\
\text { SLD in } \\
\text { third } \\
\text { through } \\
\text { fifth grade; } \\
\text { all were at } \\
\text { the inter- } \\
\text { mediate } \\
\text { level of } \\
\text { English } \\
\text { language } \\
\text { proficiency } \\
\text { and } \\
\text { Spanish as } \\
\text { L1 in a } \\
\text { Miami } \\
\text { public } \\
\text { elementary } \\
\text { school }\end{array}$ & $\begin{array}{l}\text { To } \\
\text { investigate } \\
\text { effects of } \\
\text { repeated } \\
\text { oral } \\
\text { reading } \\
\text { (English } \\
\text { only) on } \\
\text { fluency and } \\
\text { compre- } \\
\text { hension } \\
\text { using a } \\
\text { multiple } \\
\text { baseline } \\
\text { across } \\
\text { participants } \\
\text { design. }\end{array}$ & $\begin{array}{l}\text { Oral } \\
\text { repeated } \\
\text { reading with } \\
\text { corrective } \\
\text { feedback } 20 \\
\text { min per day } \\
\text { for } 10 \text { weeks }\end{array}$ & $\begin{array}{l}\text { Number of } \\
\text { words read } \\
\text { aloud correctly } \\
\text { per minute, } \\
\text { number of } \\
\text { errors per } \\
\text { minute, and } \\
\text { percentage } \\
\text { accuracy with } \\
\text { answering } \\
\text { literal compre- } \\
\text { hension } \\
\text { questions }\end{array}$ & $\begin{array}{l}\text { After } \\
\text { reading a } \\
\text { passage } \\
\text { three } \\
\text { times, } \\
\text { partic- } \\
\text { ipants } \\
\text { read } \\
\text { more } \\
\text { words, } \\
\text { made } \\
\text { fewer } \\
\text { errors, } \\
\text { and } \\
\text { improved } \\
\text { accuracy } \\
\text { in } \\
\text { answer- } \\
\text { ing literal } \\
\text { compre- } \\
\text { hension } \\
\text { (recall) } \\
\text { questions }\end{array}$ & $\begin{array}{l}\text { More } \\
\text { research is } \\
\text { needed to } \\
\text { determine } \\
\text { whether } \\
\text { repeated } \\
\text { reading } \\
\text { leads to } \\
\text { gains on } \\
\text { standardiz } \\
\text { ed } \\
\text { measures } \\
\text { of } \\
\text { compre- } \\
\text { hension or } \\
\text { gains in } \\
\text { answering } \\
\text { non-literal } \\
\text { questions } \\
\text { and to } \\
\text { determine } \\
\text { whether } \\
\text { gains } \\
\text { transfer to } \\
\text { passages } \\
\text { that are } \\
\text { read only } \\
\text { once. }\end{array}$ \\
\hline
\end{tabular}

(Table Continues) 


\begin{tabular}{|c|c|c|c|c|c|c|}
\hline Study & Participants & Purpose & $\begin{array}{l}\text { Independent } \\
\text { Variable }\end{array}$ & $\begin{array}{l}\text { Dependent } \\
\text { Variable }\end{array}$ & Results & Findings \\
\hline $\begin{array}{l}\text { Tam et } \\
\text { al., } \\
2006\end{array}$ & $\begin{array}{l}5 \text { ELs who } \\
\text { struggled in } \\
\text { reading; } \\
\text { two with } \\
\text { SLD and } \\
\text { one with } \\
\text { develop- } \\
\text { mental } \\
\text { delay; L1 } \\
\text { for one was } \\
\text { Khmer; for } \\
\text { two was } \\
\text { Spanish, } \\
\text { and for one } \\
\text { was } \\
\text { Amharic in } \\
\text { a public } \\
\text { elementary } \\
\text { school } \\
\text { where 1:1 } \\
\text { instruction } \\
\text { was } \\
\text { provided in } \\
\text { a separate } \\
\text { setting }\end{array}$ & $\begin{array}{l}\text { To analyze } \\
\text { effects of an } \\
\text { intervention } \\
\text { program that } \\
\text { uses } \\
\text { vocabulary } \\
\text { building, } \\
\text { error } \\
\text { correction, } \\
\text { and fluency } \\
\text { building on } \\
\text { the oral } \\
\text { reading rate } \\
\text { and reading } \\
\text { compre- } \\
\text { hension } \\
\text { through a } \\
\text { multiple } \\
\text { baseline } \\
\text { across } \\
\text { participants } \\
\text { design }\end{array}$ & $\begin{array}{l}\text { Individual } \\
\text { literacy } \\
\text { instruction } \\
\text { for } 45 \mathrm{~min} \\
\text { per session } \\
\text { over } 7 \text { to } 10 \\
\text { weeks }\end{array}$ & $\begin{array}{l}\text { Scores on } \\
\text { measures of } \\
\text { oral reading } \\
\text { fluency } \\
\text { (words } \\
\text { correct per } \\
\text { min and } \\
\text { errors per } \\
\text { min) }\end{array}$ & $\begin{array}{l}\text { Partic- } \\
\text { ipants } \\
\text { increased } \\
\text { oral } \\
\text { reading } \\
\text { rate; } \\
\text { improved } \\
\text { more } \\
\text { during } \\
\text { same } \\
\text { passage } \\
\text { to } \\
\text { criterion } \\
\text { condition }\end{array}$ & $\begin{array}{l}\text { Repeated } \\
\text { reading } \\
\text { with error } \\
\text { correction } \\
\text { and } \\
\text { vocabulary } \\
\text { instruction } \\
\text { warrant } \\
\text { further } \\
\text { investigatio } \\
\mathrm{n} \text { in future } \\
\text { literacy } \\
\text { interventio } \\
\mathrm{n} \text { research } \\
\text { for ELs } \\
\text { who } \\
\text { struggle in } \\
\text { reading. }\end{array}$ \\
\hline
\end{tabular}

(Table Continues) 


\begin{tabular}{|c|c|c|c|c|c|c|}
\hline Study & Participants & Purpose & $\begin{array}{l}\text { Independent } \\
\text { Variable }\end{array}$ & $\begin{array}{l}\text { Dependent } \\
\text { Variable }\end{array}$ & Results & Findings \\
\hline \multicolumn{7}{|c|}{ Phonics, Fluency, Vocabulary, and Comprehension Interventions } \\
\hline $\begin{array}{l}\text { Denton, } \\
\text { Wexler, } \\
\text { et al., } \\
2008\end{array}$ & $\begin{array}{l}38 \text { students } \\
\text { in sixth to } \\
\text { eighth } \\
\text { grade; } 33 \\
\text { received } \\
\text { special } \\
\text { education } \\
\text { services; } 22 \\
\text { were } \\
\text { identified } \\
\text { as ELs with } \\
\text { Spanish as } \\
\text { L1, all } \\
\text { were } \\
\text { struggling } \\
\text { readers in } \\
\text { an urban } \\
\text { middle } \\
\text { school in } \\
\text { the } \\
\text { southwest. } \\
\text { Small- } \\
\text { group } \\
\text { instruction } \\
\text { ( } 2 \text { to } 4 \\
\text { students) } \\
\text { took place } \\
\text { in a } \\
\text { separate }\end{array}$ & $\begin{array}{l}\text { To } \\
\text { determine } \\
\text { the extent to } \\
\text { which a } \\
\text { multi- } \\
\text { component } \\
\text { intervention } \\
\text { could } \\
\text { improve } \\
\text { outcomes in } \\
\text { word } \\
\text { reading, } \\
\text { vocabulary, } \\
\text { and compre- } \\
\text { hension } \\
\text { through a } \\
\text { pre- post } \\
\text { experiment- } \\
\text { al design }\end{array}$ & $\begin{array}{l}\text { Systematic, } \\
\text { explicit, } \\
\text { English only } \\
\text { instruction } \\
\text { in fluency } \\
\text { (repeated } \\
\text { reading), } \\
\text { compre- } \\
\text { hension } \\
\text { (collabor- } \\
\text { ative } \\
\text { strategic } \\
\text { reading), } \\
\text { decoding/ } \\
\text { encoding } \\
\text { (modified } \\
\text { Wilson), and } \\
\text { vocabulary } \\
\text { (Bringing } \\
\text { words to } \\
\text { Life) } 40 \text { min } \\
\text { per day for } \\
\text { up to } 13 \\
\text { weeks }\end{array}$ & $\begin{array}{l}\text { Scores on the } \\
\text { WJII, scores } \\
\text { to measure } \\
\text { word reading; } \\
\text { scores on the } \\
\text { dynamic } \\
\text { indicators of } \\
\text { basic early } \\
\text { literacy skills } \\
\text { (DIBELS), } \\
\text { and oral } \\
\text { reading } \\
\text { fluency } \\
\text { (ORF) words } \\
\text { correct per } \\
\text { min }\end{array}$ & $\begin{array}{l}\text { Small to } \\
\text { negative } \\
\text { effect } \\
\text { sizes on } \\
\text { all } \\
\text { measures } \\
\text {. No } \\
\text { signific- } \\
\text { ant } \\
\text { differ- } \\
\text { ences } \\
\text { between } \\
\text { treatment } \\
\text { and } \\
\text { control } \\
\text { groups; } \\
\text { corre- } \\
\text { lations } \\
\text { identified } \\
\text { between } \\
\text { teachers' } \\
\text { ratings of } \\
\text { problem } \\
\text { behavior } \\
\text { and } \\
\text { posttest } \\
\text { decoding } \\
\text { encoding } \\
\text { scores. }\end{array}$ & $\begin{array}{l}\text { Greater } \\
\text { instructional } \\
\text { intensity is } \\
\text { warranted; } \\
\text { greater } \\
\text { emphasis is } \\
\text { needed to } \\
\text { support } \\
\text { socio- } \\
\text { affective } \\
\text { factors that } \\
\text { impact } \\
\text { learning; } \\
\text { there is a } \\
\text { need for } \\
\text { culturally } \\
\text { sensitive } \\
\text { tools that } \\
\text { can assess } \\
\text { progress in } \\
\text { reading. }\end{array}$ \\
\hline
\end{tabular}

(Table Continues) 


\begin{tabular}{|c|c|c|c|c|c|c|}
\hline Study & Participants & Purpose & $\begin{array}{l}\text { Independent } \\
\text { Variable }\end{array}$ & $\begin{array}{l}\text { Dependent } \\
\text { Variable }\end{array}$ & Results & Findings \\
\hline $\begin{array}{l}\text { Graves } \\
\text { et al., } \\
2011\end{array}$ & $\begin{array}{l}58 \text { ELs in } \\
\text { sixth grade; } \\
\text { L1 not } \\
\text { reported in } \\
\text { a large, } \\
\text { urban } \\
\text { middle } \\
\text { school; } \\
\text { small- } \\
\text { group } \\
\text { instruction }\end{array}$ & $\begin{array}{l}\text { To examine } \\
\text { effects of } \\
\text { Tier II } \\
\text { intensive } \\
\text { intervent- } \\
\text { ions in } \\
\text { word } \\
\text { analysis, } \\
\text { compre- } \\
\text { hension } \\
\text { and } \\
\text { vocabulary } \\
\text { for } 20 \\
\text { hours over } \\
10 \text { weeks } \\
\text { through a } \\
\text { pre- post } \\
\text { experiment } \\
\text { al design }\end{array}$ & $\begin{array}{l}\text { Small-group } \\
\text { instruction } \\
\text { in English } \\
\text { only for } 20 \\
\text { hours across } \\
10 \text { weeks }\end{array}$ & $\begin{array}{l}\text { Scores on } \\
\text { standardized } \\
\text { measures of } \\
\text { reading } \\
\text { fluency, } \\
\text { vocabulary, } \\
\text { and compre- } \\
\text { hension }\end{array}$ & $\begin{array}{l}\text { ELs met } \\
\text { expected } \\
\text { growth } \\
\text { for } \\
\text { English- } \\
\text { only } \\
\text { students } \\
\text { (one } \\
\text { word per } \\
\text { week) on } \\
\text { oral } \\
\text { reading } \\
\text { fluency } \\
\text { measures } \\
\text { signific- } \\
\text { ant } \\
\text { growth } \\
\text { for all on } \\
\text { measures } \\
\text { of } \\
\text { fluency; } \\
\text { no differ- } \\
\text { ence on } \\
\text { measures } \\
\text { of vocab- } \\
\text { ulary and } \\
\text { compre- } \\
\text { hension }\end{array}$ & $\begin{array}{l}\text { Older } \\
\text { struggling } \\
\text { readers } \\
\text { require } \\
\text { intervention } \\
\text { of greater } \\
\text { intensity } \\
\text { (more } \\
\text { minutes per } \\
\text { session for a } \\
\text { longer } \\
\text { period of } \\
\text { time) to } \\
\text { make gains } \\
\text { on standard- } \\
\text { ized tests. }\end{array}$ \\
\hline
\end{tabular}




\begin{tabular}{lllllll}
\hline Study & Participants & Purpose & $\begin{array}{l}\text { Independent } \\
\text { Variable }\end{array}$ & $\begin{array}{l}\text { Dependent } \\
\text { Variable }\end{array}$ & Results & Findings \\
\hline Gunn & 256 & To evaluate & Supplement- & Performance & Students & Long-term \\
et al., & students in & effects of & al & on & receiving & supple- \\
2000 & Kinder- & English & instruction & standardized & supple- & mental \\
& garten & only & for 25-30 & measures of & mental & instruction \\
& through & instruction & min per day & early literacy & instruc- & is \\
& third grade; & in phono- & for 5 months & skills & tion & necessary \\
& 19 were & logical & & (DIBELS), & made & in order to \\
& ELs with & awareness & & oral reading & gains & identify \\
& Spanish as & and & & fluency, and & over the & treatment \\
their L1in & decoding & & compre- & compar- & effects. \\
& Oregon; & skills on & & hension & ison & Explicit \\
& small- & measures & & (WJRIII) & group. & instruction \\
group & of oral & & & No & in decoding \\
instruction & reading & & & differ- & in English \\
of up to & fluency, & & ences in & benefits \\
three & vocabulary & & perform- & students \\
students & and & & ances & who are \\
& compre- & & were & learning \\
& hension & detected & English as \\
& through a & & based on & a second \\
& pre- post & & level of & language. \\
& experiment & & English & \\
& -al design & & proficien & \\
& & & cy &
\end{tabular}




\begin{tabular}{|c|c|c|c|c|c|c|}
\hline Study & Participants & Purpose & $\begin{array}{l}\text { Independent } \\
\text { Variable }\end{array}$ & $\begin{array}{l}\text { Dependent } \\
\text { Variable }\end{array}$ & Results & Findings \\
\hline $\begin{array}{l}\text { Gunn } \\
\text { et al., } \\
2002\end{array}$ & $\begin{array}{l}\text { Struggling } \\
\text { readers in } \\
\text { Kinder- } \\
\text { garten } \\
\text { through } \\
\text { second } \\
\text { grade, } \\
\text { including } \\
16 \text { ELs } \\
\text { with } \\
\text { Spanish as } \\
\text { L1 in } \\
\text { Oregon; } \\
\text { small- } \\
\text { group } \\
\text { instruction } \\
\text { of up to } 3 \\
\text { students }\end{array}$ & $\begin{array}{l}\text { To follow } \\
\text { up and } \\
\text { determine } \\
\text { results of } \\
\text { English } \\
\text { only } \\
\text { supplement } \\
\text {-al reading } \\
\text { instruction } \\
\text { in basic } \\
\text { decoding } \\
\text { and } \\
\text { compre- } \\
\text { hension } \\
\text { through a } \\
\text { pre- post } \\
\text { experiment } \\
\text { al design. }\end{array}$ & $\begin{array}{l}\text { Supplement- } \\
\text { al } \\
\text { instruction } \\
\text { for } 30 \text { min } \\
\text { per day for } 5 \\
\text { months in } \\
\text { year one in } \\
10 \text { months } \\
\text { in year two }\end{array}$ & $\begin{array}{l}\text { Scores on } \\
\text { measures of } \\
\text { early literacy } \\
\text { skills } \\
\text { (DIBELS), } \\
\text { oral reading } \\
\text { fluency, and } \\
\text { compre- } \\
\text { hension } \\
\text { (WJIII-R) }\end{array}$ & $\begin{array}{l}\text { The } \\
\text { group } \\
\text { receiving } \\
\text { supple- } \\
\text { mental } \\
\text { instruct- } \\
\text { tion } \\
\text { made } \\
\text { gains } \\
\text { over the } \\
\text { control } \\
\text { group } \\
\text { and } \\
\text { maintain- } \\
\text { ed their } \\
\text { improve- } \\
\text { ments } \\
\text { over } \\
\text { time. }\end{array}$ & $\begin{array}{l}\text { Direct and } \\
\text { explicit } \\
\text { supple- } \\
\text { mental } \\
\text { instruction } \\
\text { leads to } \\
\text { gains in } \\
\text { early } \\
\text { literacy } \\
\text { skills } \\
\text { (phonemic } \\
\text { awareness } \\
\text { and } \\
\text { decoding) } \\
\text { that lead } \\
\text { to } \\
\text { improve- } \\
\text { ments in } \\
\text { fluency } \\
\text { and } \\
\text { compre- } \\
\text { hension. }\end{array}$ \\
\hline
\end{tabular}

(Table Continues) 


\begin{tabular}{|c|c|c|c|c|c|c|}
\hline Study & Participants & Purpose & $\begin{array}{l}\text { Independent } \\
\text { Variable }\end{array}$ & $\begin{array}{l}\text { Dependent } \\
\text { Variable }\end{array}$ & Results & Findings \\
\hline $\begin{array}{l}\text { Linan- } \\
\text { Thomp } \\
\text {-son, et } \\
\text { al., } \\
2003\end{array}$ & $\begin{array}{l}26 \text { ELs in } \\
\text { second } \\
\text { grade with } \\
\text { Spanish as } \\
\text { L1 in an } \\
\text { urban Title } \\
1 \\
\text { elementary } \\
\text { school in } \\
\text { the } \\
\text { southwest }\end{array}$ & $\begin{array}{l}\text { To examine } \\
\text { effects of a } \\
\text { supple- } \\
\text { mental } \\
\text { reading } \\
\text { interven- } \\
\text { tion for } \\
\text { struggling } \\
\text { readers } \\
\text { through an } \\
\text { exploratory } \\
\text { study. }\end{array}$ & $\begin{array}{l}13 \text { weeks of } \\
\text { English only } \\
\text { supplementa } \\
1 \text { instruction } \\
\text { for } 30 \text { min } \\
\text { per day; } 1: 1 \\
\text { or two to } \\
\text { three per } \\
\text { small group } \\
\text { over } 58 \\
\text { sessions }\end{array}$ & $\begin{array}{l}\text { Scores on } \\
\text { measures } \\
\text { of compre- } \\
\text { hension } \\
\text { (WRMT- } \\
\text { R), fluency, } \\
\text { and } \\
\text { phonologic } \\
\text { al } \\
\text { awareness }\end{array}$ & $\begin{array}{l}\text { Strong } \\
\text { results on } \\
\text { passage } \\
\text { compre- } \\
\text { hension } \\
\text { subtest of } \\
\text { Wood- } \\
\text { cock } \\
\text { Reading } \\
\text { Mastery } \\
\text { Test } \\
\text { (WRMT- } \\
\text { R); after } \\
4 \text { weeks, } \\
\text { gains } \\
\text { main- } \\
\text { tained }\end{array}$ & $\begin{array}{l}\text { Oral } \\
\text { reading } \\
\text { fluency } \\
\text { measures } \\
\text { did not } \\
\text { reveal } \\
\text { gains; } \\
\text { norms for } \\
\text { ELs are } \\
\text { not } \\
\text { available } \\
\text { to } \\
\text { determine } \\
\text { what } \\
\text { expected } \\
\text { growth } \\
\text { should be; } \\
\text { improved } \\
\text { comprehe } \\
\text { nsion led } \\
\text { to slower } \\
\text { rates of } \\
\text { oral } \\
\text { reading } \\
\text { fluency. }\end{array}$ \\
\hline
\end{tabular}

(Table Continues) 


\begin{tabular}{|c|c|c|c|c|c|c|}
\hline Study & Participants & Purpose & $\begin{array}{l}\text { Independent } \\
\text { Variable }\end{array}$ & $\begin{array}{l}\text { Dependent } \\
\text { Variable }\end{array}$ & Results & Findings \\
\hline $\begin{array}{l}\text { Mon- } \\
\text { toya, } \\
2008\end{array}$ & $\begin{array}{l}4 \text { ELs with } \\
\text { high } \\
\text { incidence } \\
\text { disabilities } \\
\text { in sixth } \\
\text { grade; } \\
\text { Spanish } \\
\text { was L1 for } \\
\text { all } \\
\text { participants } \\
\text { in a rural } \\
\text { public } \\
\text { school in } \\
\text { southern } \\
\text { California }\end{array}$ & $\begin{array}{l}\text { To } \\
\text { investigate } \\
\text { effects of } \\
\text { English } \\
\text { only guided } \\
\text { reading } \\
\text { instruction } \\
\text { on reading } \\
\text { compre- } \\
\text { hension } \\
\text { through a } \\
\text { multiple } \\
\text { baseline } \\
\text { across } \\
\text { participants }\end{array}$ & $\begin{array}{l}30 \text { days of } \\
\text { instruction } \\
\text { in English } \\
\text { only to } \\
\text { guide } \\
\text { compre- } \\
\text { hension } \\
\text { before, } \\
\text { during, and } \\
\text { after reading }\end{array}$ & $\begin{array}{l}\text { Scores on } \\
\text { curriculum- } \\
\text { based } \\
\text { assessments } \\
\text { modeled after } \\
\text { the } \\
\text { MacMillan/ } \\
\text { McGraw Hill } \\
\text { "Spotlight on } \\
\text { Literacy } \\
\text { Assessment" } \\
\text { and scores on } \\
\text { a retelling } \\
\text { instrument }\end{array}$ & $\begin{array}{l}\text { Data } \\
\text { depicted } \\
\text { a high } \\
\text { level of } \\
\text { variabil- } \\
\text { ity with a } \\
\text { high } \\
\text { percent- } \\
\text { age of } \\
\text { over- } \\
\text { lapping } \\
\text { data } \\
\text { points for } \\
\text { three } \\
\text { students } \\
\text { on the } \\
\text { retelling } \\
\text { rubric } \\
\text { and for } \\
\text { two } \\
\text { students } \\
\text { on the } \\
\text { compre- } \\
\text { hension } \\
\text { assess- } \\
\text { ment }\end{array}$ & $\begin{array}{l}\text { Integra- } \\
\text { tion of } \\
\text { language } \\
\text { supports } \\
\text { (ESL } \\
\text { strategies, } \\
\text { oral } \\
\text { language } \\
\text { support, } \\
\text { and } \\
\text { vocab- } \\
\text { ulary } \\
\text { support) } \\
\text { are needed } \\
\text { in future } \\
\text { research; } \\
\text { additional } \\
\text { techniques } \\
\text { are needed } \\
\text { to ensure } \\
\text { that } \\
\text { students } \\
\text { retain } \\
\text { reading } \\
\text { compre- } \\
\text { hension } \\
\text { strategies. }\end{array}$ \\
\hline
\end{tabular}

(Table Continues) 


\begin{tabular}{|c|c|c|c|c|c|c|}
\hline Study & Participants & Purpose & $\begin{array}{l}\text { Independent } \\
\text { Variable }\end{array}$ & $\begin{array}{l}\text { Dependent } \\
\text { Variable }\end{array}$ & Results & Findings \\
\hline $\begin{array}{l}\text { San- } \\
\text { toro } \\
\text { et al., } \\
2006\end{array}$ & $\begin{array}{l}4 \text { ELs in } \\
\text { second } \\
\text { grade with } \\
\text { low reading } \\
\text { achieve- } \\
\text { ment (one } \\
\text { was } \\
\text { eligible for } \\
\text { special } \\
\text { education } \\
\text { and one } \\
\text { was in the } \\
\text { evaluation } \\
\text { process); } \\
\text { L1 was not } \\
\text { reported in } \\
\text { an } \\
\text { elementary } \\
\text { school in } \\
\text { the } \\
\text { northeast } \\
\text { with } 1: 1 \\
\text { instruction } \\
\text { in a } \\
\text { separate } \\
\text { setting }\end{array}$ & $\begin{array}{l}\text { To } \\
\text { investigate } \\
\text { the } \\
\text { effective- } \\
\text { ness and } \\
\text { feasibility } \\
\text { of Read } \\
\text { Well, in } \\
\text { English } \\
\text { only, with } \\
\text { specific } \\
\text { emphasis } \\
\text { on the } \\
\text { compre- } \\
\text { hension } \\
\text { component } \\
\text { of the } \\
\text { program } \\
\text { through a } \\
\text { multiple } \\
\text { probe } \\
\text { across } \\
\text { participants } \\
\text { design }\end{array}$ & $\begin{array}{l}\text { Read Well (a } \\
\text { commercial } \\
\text { intervention } \\
\text { for } \\
\text { beginning } \\
\text { readers or } \\
\text { for } \\
\text { remediation } \\
\text { with second- } \\
\text { grade } \\
\text { monolingual } \\
\text { struggling } \\
\text { readers) } 30 \\
\text { min per day } \\
\text { from } 8 \text { to } 11 \\
\text { weeks }\end{array}$ & $\begin{array}{l}\text { Scores on: } \\
\text { DIBELS } \\
\text { phonological } \\
\text { awareness } \\
\text { and } \\
\text { alphabetic } \\
\text { principle } \\
\text { assessments; } \\
\text { WRMT-R } \\
\text { word } \\
\text { identification, } \\
\text { word attack, } \\
\text { and passage } \\
\text { compre- } \\
\text { hension } \\
\text { subtests; } \\
\text { number of } \\
\text { words correct } \\
\text { per min on } \\
\text { oral reading } \\
\text { fluency } \\
\text { measures }\end{array}$ & $\begin{array}{l}\text { Minimal } \\
\text { effects } \\
\text { compre- } \\
\text { hension } \\
\text { at } \\
\text { posttest. } \\
\text { Two } \\
\text { partici- } \\
\text { pants } \\
\text { made } \\
\text { gains and } \\
\text { two } \\
\text { regressed } \\
\text {. }\end{array}$ & $\begin{array}{l}\text { Intensive, } \\
\text { individual, } \\
\text { explicit, } \\
\text { and } \\
\text { systematic } \\
\text { instruction } \\
\text { led to } \\
\text { gains in } \\
\text { decoding } \\
\text { but not } \\
\text { compre- } \\
\text { hension; } \\
\text { specific } \\
\text { focus on } \\
\text { compre- } \\
\text { hension } \\
\text { strategies } \\
\text { is needed. }\end{array}$ \\
\hline
\end{tabular}

(Table Continues) 


\begin{tabular}{|c|c|c|c|c|c|c|}
\hline Study & Participants & Purpose & $\begin{array}{l}\text { Independent } \\
\text { Variable }\end{array}$ & $\begin{array}{l}\text { Dependent } \\
\text { Variable }\end{array}$ & Results & Findings \\
\hline $\begin{array}{l}\text { Vau- } \\
\text { ghn, } \\
\text { Linan- } \\
\text { Thomp } \\
\text {-son, et } \\
\text { al., } \\
2006\end{array}$ & $\begin{array}{l}69 \text { first- } \\
\text { grade } \\
\text { students } \\
\text { who use } \\
\text { Spanish as } \\
\text { L1 in high } \\
\text { rating } \\
\text { schools in } \\
\text { Texas }\end{array}$ & $\begin{array}{l}\text { To invest- } \\
\text { igate how } \\
\text { an } \\
\text { interven- } \\
\text { tion in } \\
\text { Spanish } \\
\text { would } \\
\text { influence } \\
\text { outcomes } \\
\text { on } \\
\text { Spanish } \\
\text { reading, } \\
\text { English } \\
\text { reading, } \\
\text { and } \\
\text { Spanish } \\
\text { oral } \\
\text { language } \\
\text { skills } \\
\text { through a } \\
\text { pre- post } \\
\text { experi- } \\
\text { mental } \\
\text { design }\end{array}$ & $\begin{array}{l}\text { Small } \\
\text { groups of } \\
\text { three to five } \\
\text { per one } \\
\text { bilingual } \\
\text { intervention- } \\
\text { ist; } 50 \text { min } \\
\text { per day for } 8 \\
\text { months; } \\
\text { instruction } \\
\text { focused on } \\
\text { alphabetic } \\
\text { principle, } \\
\text { reading } \\
\text { connected } \\
\text { text, oral } \\
\text { language, } \\
\text { and } \\
\text { vocabulary }\end{array}$ & $\begin{array}{l}\text { Scores on } \\
\text { Spanish/ } \\
\text { English } \\
\text { measures: } \\
\text { WJRIII, } \\
\text { DIBELS, } \\
\text { Woodcock } \\
\text { Language } \\
\text { Proficiency } \\
\text { Battery - } \\
\text { Revised } \\
\text { (WLPB-R) } \\
\text { and } \\
\text { comprehend- } \\
\text { sive test of } \\
\text { phonological } \\
\text { processing } \\
\text { (CTOPP) }\end{array}$ & $\begin{array}{l}\text { Treat- } \\
\text { ment } \\
\text { group } \\
\text { outper- } \\
\text { formed } \\
\text { control } \\
\text { group on } \\
\text { word } \\
\text { attack } \\
\text { and } \\
\text { phono- } \\
\text { logical } \\
\text { process- } \\
\text { ing in } \\
\text { English; } \\
\text { on } \\
\text { measures } \\
\text { in } \\
\text { Spanish, } \\
\text { the } \\
\text { treatment } \\
\text { group } \\
\text { outper- } \\
\text { formed } \\
\text { the } \\
\text { control } \\
\text { group on } \\
\text { all } \\
\text { measures }\end{array}$ & $\begin{array}{l}\text { Interven- } \\
\text { tion } \\
\text { instruction } \\
\text { that } \\
\text { focuses on } \\
\text { vocab- } \\
\text { ulary and } \\
\text { oral } \\
\text { language } \\
\text { develop- } \\
\text { ment lead } \\
\text { to } \\
\text { improved } \\
\text { outcomes } \\
\text { on the } \\
\text { WLPB-R. }\end{array}$ \\
\hline
\end{tabular}




\begin{tabular}{|c|c|c|c|c|c|c|}
\hline Study & Participants & Purpose & $\begin{array}{l}\text { Independent } \\
\text { Variable }\end{array}$ & $\begin{array}{l}\text { Dependent } \\
\text { Variable }\end{array}$ & Results & Findings \\
\hline $\begin{array}{l}\text { Vau- } \\
\text { ghn, } \\
\text { Mathes } \\
\text {, et al., } \\
2006\end{array}$ & $\begin{array}{l}41 \text { ELs in } \\
\text { first grade } \\
\text { who were } \\
\text { "at risk" } \\
\text { for school } \\
\text { failure and } \\
\text { who used } \\
\text { Spanish as } \\
\text { L1 in high } \\
\text { rating } \\
\text { schools in } \\
\text { Texas }\end{array}$ & $\begin{array}{l}\text { To } \\
\text { examine } \\
\text { effects of } \\
\text { systematic } \\
\text {, explicit } \\
\text { instruction } \\
\text { in English } \\
\text { oral } \\
\text { language } \\
\text { by trained } \\
\text { bilingual } \\
\text { teachers in } \\
\text { a pre- post } \\
\text { experi- } \\
\text { mental } \\
\text { design }\end{array}$ & $\begin{array}{l}\text { Small-group } \\
\text { instruction } \\
\text { (three to } \\
\text { five) in } \\
\text { English } \\
\text { (with } \\
\text { Spanish } \\
\text { support as } \\
\text { needed) for } \\
50 \text { min per } \\
\text { day for } \\
\text { seven } \\
\text { months }\end{array}$ & $\begin{array}{l}\text { Scores on } \\
\text { multiple } \\
\text { measures in } \\
\text { Spanish and } \\
\text { English: } \\
\text { DIBELS, } \\
\text { WLPB-R, } \\
\text { and CTOPP }\end{array}$ & $\begin{array}{l}\text { Treat- } \\
\text { ment } \\
\text { group } \\
\text { outper- } \\
\text { formed } \\
\text { control } \\
\text { on all } \\
\text { measures } \\
\text {, with } \\
\text { most } \\
\text { signif- } \\
\text { icant } \\
\text { results in } \\
\text { compre- } \\
\text { hension; } \\
\text { in L1, } \\
\text { there was } \\
\text { no signif- } \\
\text { icant } \\
\text { differ- } \\
\text { ence } \\
\text { between } \\
\text { treatment } \\
\text { and } \\
\text { control }\end{array}$ & $\begin{array}{l}\text { At risk } \\
\text { ELs } \\
\text { benefit } \\
\text { from } \\
\text { intensive } \\
\text { instruction } \\
\text { in English } \\
\text { that } \\
\text { focuses on } \\
\text { phonemic } \\
\text { awareness } \\
\text {, letter } \\
\text { know- } \\
\text { ledge, } \\
\text { alphabetic } \\
\text { decoding, } \\
\text { decodable } \\
\text { text } \\
\text { practice, } \\
\text { and } \\
\text { compre- } \\
\text { hension } \\
\text { strategies; } \\
\text { in the } \\
\text { future } \\
\text { emphasis } \\
\text { on vocab- } \\
\text { ulary } \\
\text { building, } \\
\text { using } \\
\text { schema, } \\
\text { and ESL } \\
\text { strategies } \\
\text { added. }\end{array}$ \\
\hline
\end{tabular}

(Table Continues) 


\begin{tabular}{|c|c|c|c|c|c|c|}
\hline Study & Participants & Purpose & $\begin{array}{l}\text { Independent } \\
\text { Variable }\end{array}$ & $\begin{array}{l}\text { Dependent } \\
\text { Variable }\end{array}$ & Results & Findings \\
\hline \multicolumn{7}{|c|}{ Phonics Compared with Listening Comprehension } \\
\hline $\begin{array}{l}\text { Solari } \\
\& \\
\text { Gerber } \\
, 2008\end{array}$ & $\begin{array}{l}82 \text { ELs in } \\
\text { Kinder- } \\
\text { garten } \\
\text { (some "at } \\
\text { risk" and } \\
\text { others "not } \\
\text { at risk"); } \\
\text { Spanish } \\
\text { was L1 in a } \\
\text { Title } 1 \\
\text { school in } \\
\text { California }\end{array}$ & $\begin{array}{l}\text { To invest- } \\
\text { igate } \\
\text { effects of } \\
\text { three } \\
\text { methods } \\
\text { of } \\
\text { instruction } \\
\text { (in } \\
\text { English } \\
\text { only) on } \\
\text { precursors } \\
\text { to } \\
\text { successful } \\
\text { reading } \\
\text { through a } \\
\text { pre- post } \\
\text { experi- } \\
\text { mental } \\
\text { study }\end{array}$ & $\begin{array}{l}20 \text { min per } \\
\text { day for } 8 \\
\text { weeks; } \\
\text { small- group } \\
\text { instruction } \\
\text { in either } \\
\text { phono- } \\
\text { logical } \\
\text { awareness, } \\
\text { listening } \\
\text { compre- } \\
\text { hension, or } \\
\text { phono- } \\
\text { logical } \\
\text { awareness } \\
\text { combined } \\
\text { with } \\
\text { listening } \\
\text { compre- } \\
\text { hension }\end{array}$ & $\begin{array}{l}\text { Scores on } \\
\text { multiple } \\
\text { measures: } \\
\text { WJRIII, } \\
\text { Peabody } \\
\text { Picture } \\
\text { Vocabulary } \\
\text { Test (PPVT), } \\
\text { CTOPP, and } \\
\text { measures of } \\
\text { phonological } \\
\text { awareness } \\
\text { (rime } \\
\text { detection and } \\
\text { onset } \\
\text { detection) }\end{array}$ & $\begin{array}{l}\text { All } \\
\text { improved } \\
\text { in phono- } \\
\text { logical } \\
\text { aware- } \\
\text { ness; at } \\
\text { risk } \\
\text { students } \\
\text { in the } \\
\text { listening } \\
\text { compre- } \\
\text { hension } \\
\text { group } \\
\text { out- } \\
\text { perform- } \\
\text { ed all } \\
\text { other } \\
\text { groups. }\end{array}$ & $\begin{array}{l}\text { Direct } \\
\text { instruction } \\
\text { on early } \\
\text { word- } \\
\text { level skills } \\
\text { is } \\
\text { effective } \\
\text { for ELs } \\
\text { when a } \\
\text { listening } \\
\text { compre- } \\
\text { hension } \\
\text { compon- } \\
\text { ent is } \\
\text { empha- } \\
\text { sized; } \\
\text { increases } \\
\text { in } \\
\text { listening } \\
\text { compre- } \\
\text { hension } \\
\text { led to } \\
\text { increases } \\
\text { in phono- } \\
\text { logical } \\
\text { awareness } \\
\text {; small } \\
\text { homo- } \\
\text { geneous } \\
\text { groups } \\
\text { gave } \\
\text { multiple } \\
\text { opportune } \\
\text {-ities to } \\
\text { respond. }\end{array}$ \\
\hline
\end{tabular}

(Table Continues) 


\begin{tabular}{|c|c|c|c|c|c|c|}
\hline Study & Participants & Purpose & $\begin{array}{l}\text { Independent } \\
\text { Variable }\end{array}$ & $\begin{array}{l}\text { Dependent } \\
\text { Variable }\end{array}$ & Results & Findings \\
\hline \multicolumn{7}{|c|}{ Phonics and Comprehension Compared with Fluency and Comprehension } \\
\hline $\begin{array}{l}\text { Denton } \\
\text {, An- } \\
\text { thony, } \\
\text { et al., } \\
2004\end{array}$ & $\begin{array}{l}93 \text { students } \\
\text { who were } \\
\text { designated } \\
\text { by teachers } \\
\text { as low- } \\
\text { achieving; } \\
\text { all used } \\
\text { Spanish as } \\
\text { L1 in an } \\
\text { elementary } \\
\text { school in } \\
\text { central } \\
\text { Texas; } \\
\text { tutoring } \\
\text { took place } \\
\text { in a } \\
\text { separate } \\
\text { setting }\end{array}$ & $\begin{array}{l}\text { To } \\
\text { evaluate } \\
\text { effects of } \\
\text { two } \\
\text { English } \\
\text { literacy } \\
\text { interven- } \\
\text { tions on } \\
\text { reading } \\
\text { progress } \\
\text { of } \\
\text { Spanish- } \\
\text { dominant } \\
\text { bilingual } \\
\text { students } \\
\text { learning to } \\
\text { read in } \\
\text { English } \\
\text { through a } \\
\text { pre- post } \\
\text { experi- } \\
\text { mental } \\
\text { design }\end{array}$ & $\begin{array}{l}\text { One-to-one } \\
\text { instruction } \\
\text { for } 40 \text { min } \\
\text { per day for } \\
10 \text { weeks } \\
\text { using either } \\
\text { Read Well } \\
\text { or modified } \\
\text { Read } \\
\text { Naturally }\end{array}$ & $\begin{array}{l}\text { Scores on } \\
\text { subtests of } \\
\text { Woodcock } \\
\text { Reading } \\
\text { Mastery Test } \\
\text {-Revised } \\
\text { (WRMT-R): } \\
\text { word } \\
\text { identification, } \\
\text { word attack, } \\
\text { passage } \\
\text { compre- } \\
\text { hension }\end{array}$ & $\begin{array}{l}\text { No } \\
\text { change in } \\
\text { word } \\
\text { identific- } \\
\text { ation } \\
\text { scores } \\
\text { for the } \\
\text { control } \\
\text { group, } \\
\text { but the } \\
\text { Read } \\
\text { Well } \\
\text { group } \\
\text { increased } \\
\text { on this } \\
\text { subtest; } \\
\text { there was } \\
\text { no } \\
\text { statistic- } \\
\text { ally } \\
\text { signifi- } \\
\text { cant } \\
\text { differ- } \\
\text { ence } \\
\text { between } \\
\text { any } \\
\text { scores } \\
\text { for } \\
\text { treatment } \\
\text { and } \\
\text { interven- } \\
\text { tion for } \\
\text { the Read } \\
\text { Naturally } \\
\text { group }\end{array}$ & $\begin{array}{l}\text { Read Well } \\
\text { provided } \\
\text { frequent } \\
\text { repetition, } \\
\text { multiple } \\
\text { opportunit } \\
\text { ies for } \\
\text { practice, } \\
\text { and } \\
\text { immediate } \\
\text { perform- } \\
\text { ance } \\
\text { feedback; } \\
\text { with } \\
\text { modifica- } \\
\text { tions Read } \\
\text { Naturally } \\
\text { empha- } \\
\text { sized } \\
\text { vocabu- } \\
\text { lary and } \\
\text { decoding } \\
\text { but more } \\
\text { emphasis } \\
\text { is needed } \\
\text { on } \\
\text { compre- } \\
\text { hension } \\
\text { strategies. }\end{array}$ \\
\hline
\end{tabular}

(Table Continues) 


\begin{tabular}{|c|c|c|c|c|c|c|}
\hline Study & Participants & Purpose & $\begin{array}{l}\text { Independent } \\
\text { Variable }\end{array}$ & $\begin{array}{l}\text { Dependent } \\
\text { Variable }\end{array}$ & Results & Findings \\
\hline $\begin{array}{l}\text { Wan- } \\
\text { zek \& } \\
\text { Rob- } \\
\text { erts, } \\
2012\end{array}$ & $\begin{array}{l}87 \text { fourth- } \\
\text { grade } \\
\text { students } \\
\text { with } \\
\text { reading } \\
\text { difficulties, } \\
\text { including } \\
54 \text { ELs; L1 } \\
\text { not } \\
\text { reported in } \\
\text { an } \\
\text { elementary } \\
\text { school in } \\
\text { the } \\
\text { southwest. }\end{array}$ & $\begin{array}{l}\text { To } \\
\text { investing- } \\
\text { ate effects } \\
\text { of three } \\
\text { treatments } \\
\text { on } \\
\text { measures } \\
\text { of word } \\
\text { reading, } \\
\text { fluency, } \\
\text { vocab- } \\
\text { ulary, and } \\
\text { compre- } \\
\text { hension } \\
\text { through a } \\
\text { pre- post } \\
\text { experi- } \\
\text { mental } \\
\text { design }\end{array}$ & $\begin{array}{l}\text { Small-group } \\
\text { instruction, } \\
30 \text { min per } \\
\text { day over } 28 \\
\text { weeks; } \\
\text { treatment } \\
\text { involved } \\
\text { either a } \\
\text { modified } \\
\text { version of } \\
\text { Wilson } \\
\text { (systematic } \\
\text { phonics } \\
\text { instruction); } \\
\text { collaborative } \\
\text { strategic } \\
\text { reading } \\
\text { (CSR); or a } \\
\text { responsive } \\
\text { intervention, } \\
\text { consisting of } \\
\text { Wilson or } \\
\text { CSR }\end{array}$ & $\begin{array}{l}\text { Scores on the } \\
\text { WJIII: word } \\
\text { identification, } \\
\text { word attack, } \\
\text { listening } \\
\text { compre- } \\
\text { hension, and } \\
\text { passage } \\
\text { compre- } \\
\text { hension }\end{array}$ & $\begin{array}{l}\text { ELs } \\
\text { performe } \\
d \text { better } \\
\text { than non- } \\
\text { ELs in } \\
\text { all } \\
\text { treatment } \\
\text { groups } \\
\text { on all } \\
\text { measures } \\
\text {; } \\
\text { otherwis } \\
\text { e there } \\
\text { were no } \\
\text { statistic- } \\
\text { ally } \\
\text { signif- } \\
\text { icant } \\
\text { differenc } \\
\text { es } \\
\text { between } \\
\text { outcomes } \\
\text { from any } \\
\text { group }\end{array}$ & $\begin{array}{l}\text { Small } \\
\text { effects can } \\
\text { be } \\
\text { obtained } \\
\text { for } \\
\text { students in } \\
\text { inter- } \\
\text { ventions } \\
\text { that focus } \\
\text { on word } \\
\text { recog- } \\
\text { nition; no } \\
\text { effects } \\
\text { were } \\
\text { document- } \\
\text { ed for } \\
\text { compre- } \\
\text { hension } \\
\text { instruction } \\
\text { that } \\
\text { empha- } \\
\text { sized } \\
\text { collabor- } \\
\text { ative } \\
\text { conver- } \\
\text { sations } \\
\text { and } \\
\text { compre- } \\
\text { hension } \\
\text { strategy } \\
\text { instruction }\end{array}$ \\
\hline
\end{tabular}




\section{Explicit Strategy Instruction with a Self-Monitoring Procedure}

To combine the goal of teaching students how and why to use strategies with the goal of promoting self-directedness in applying strategies, researchers have historically integrated strategy instruction with self-monitoring procedures in studies on behavior interventions (Lam, Cole, \& Shapiro, 1994). Over the past three decades, the effectiveness of self-monitoring techniques for students with learning, emotional, or behavioral disabilities has been demonstrated in several studies that also involved using reading comprehension strategies to summarize or find the main idea of textual information (Chan, 1991; Graves, 1986; Graves \& Levin, 1989; Jitendra et al., 1998; Jitendra et al., 2000; Malone \& Mastropieri, 1992). The generalizability of reading comprehension strategies and self-monitoring techniques was assessed in only two studies, first by fading self-monitoring and reading comprehension strategy instruction and then by having participants read on-grade-level text (Jitendra et al., 2000; Malone \& Mastropieri, 1992). In both studies, participants with SLD who received the intervention outperformed participants in the control conditions on selection tasks (e.g., multiple choice questions about the main idea of the text). Although both studies sought to examine whether effects of instruction would be maintained over time, no information (e.g., formative assessment data) was provided to confirm whether all participants in treatment conditions had internalized the strategies prior to moving into generalization and maintenance phases of the studies.

The main idea strategy in Jitendra et al. (2000) involved having participants (33 middle school students) select or produce the main idea of a researcher-developed 
passage by identifying the most important thing, person, or action. Then, participants were taught to identify where, when, how, and why the information related to the passage. Throughout instruction, participants were taught to use a four-step strategy: (a) read, (b) recall the strategy from the prompt card, (c) use the strategy, and (d) identify or write the main idea. Instruction took place in small, homogenous groups (of six to eight students) and lasted up to 40 min per session for 15 days. Researchers developed passages of three to five sentences for use during instruction. All passages were written below grade level to match the instructional reading levels of participants, with the exception of the passages used to assess the generalizability of the main idea strategy with self-monitoring procedures. Performance was assessed on researcher-developed measures, which included multiple-choice and short-answer questions. For participants with learning difficulties in the treatment condition, performance on multiple-choice items consistently exceeded performance on short-answer questions. Participants in the treatment condition outperformed participants in the control condition on selection tasks immediately after treatment and again after a 6 -week delay $(E S=2.15)$. No betweengroup difference was observed in performance on short-answer tasks after the 6-week delay. Participants in the treatment condition reported favorable perceptions of the strategy, indicating that it was helpful and easy to understand.

Jitendra et al. (2000) did not provide enough information to determine whether the main idea strategies and self-monitoring procedures were internalized by participants prior to administering the generalization and maintenance measures. For example, no information was provided to describe how long (e.g., number of sessions) it took for 
participants in the treatment condition to internalize the comprehension strategy and the self-monitoring procedure. Therefore, it is not possible to determine whether participants in the treatment condition were able to activate the main idea strategy and mindfully execute the steps of the self-monitoring procedure prior to the posttest and delayed posttest. Jitendra et al. (2000) provided results to suggest that students with SLD, albeit a small sample, can maintain and generalize reading comprehension strategies and selfmonitoring procedures, at least to the extent that manifests on selection tasks. Ultimately, given the criticism related to generalization and maintenance of explicit reading comprehension strategy instruction and given the potential for self-monitoring procedures to promote internalization, there is a need for research to uncover what it takes for strategies and procedures to be internalized by struggling readers with SLD (e.g., total number of sessions and number of min per session when teacher-mediated, small-group instruction is provided).

\section{Summary}

Over the past 35 years, several approaches to reading comprehension instruction have been found effective for students with SLD. Explicit instruction, or directly teaching students to apply strategies through modeling, guided practice, and independent practice, plays a vital role in each of these approaches. Compared to the literature base on improving reading for students with SLD, very few studies (i.e., 20) have been conducted to empirically validate interventions for improving reading comprehension for ELs with SLD (Bos \& Anders, 1990; Jiménez, 1997; Klingner \& Vaughn, 1992; Sáenz, Fuchs, \& Fuchs, 2005; Santoro, Jitendra, Starosta, \& Sacks, 2006; Tam, Heward, \& 
Heng, 2006). Evidence from intervention studies aimed to improve reading comprehension for ELs with SLD supports key findings that overlap with findings from reading comprehension research for monolingual students with SLD. For example, using peer-mediated instruction and explicitly teaching comprehension strategies are methods that have led to gains in reading comprehension performance and achievement. Given that some commonalities underlie reading difficulties experienced by native and nonnative English speakers with SLD, such as difficulties with working memory, it makes sense to explore the possibility that interventions that have been found effective for native English speakers with SLD can be modified to meet needs of ELs with SLD. To determine effectiveness of interventions for culturally and linguistically diverse students with SLD, there is a need to expand the current research base, as very few studies (Jiménez, 1997; Klingner \& Vaughn, 1996; Sáenz et al., 2005) illustrate how to improve reading outcomes for struggling readers who have SLD and who are not fully proficient in English.

To meet needs of monolingual students with SLD, effective reading comprehension interventions include providing text enhancements, such as using semantic maps or graphic organizers. Text enhancements are found to be most effective when students are taught why, how, and when to use these tools. Additionally, peermediated reading comprehension strategy instruction, such as working in groups through the collaborative strategic reading model, is effective for students with SLD. Research on class-wide peer tutoring and peer-assisted learning strategies reveals that students, with and without disabilities, benefit from this model of reading instruction. Finally, 
teacher-mediated explicit instruction in comprehension strategies is effective for students with SLD, particularly when self-instructional techniques are a central focus of instruction. When explicit instruction is combined with self-monitoring procedures, students with SLD can attain self-regulation of strategy use to facilitate reading for meaning (Gajria, Jitendra, Sood, \& Sacks, 2007; Kim et al., 2012).

\section{Synthesis and Conclusions}

The current research base on reading comprehension interventions for students with SLD does not contain a sufficient number of rigorous studies to establish an evidence base for ELs with SLD. It contains three studies with enough methodological rigor to merit knowledge claims (Helman, Calhoon, \& Kern, 2014; Klingner \& Vaughn, 1996; Sáenz et al., 2005); however none of these studies has been replicated in other settings with other participants. Moreover, the majority of reading comprehension intervention experimental studies that included culturally and linguistically diverse participants used a culture-free approach, or one that did not consider the fundamental aspects of culture and linguistic diversity that participants, interventionists, and researchers brought to the study (Albers \& Hoffman, 2012; Bos \& Anders, 1992; Denton, Anthony, et al., 2004; Denton, Wexler, et al, 2008; Graves et al., 2011; Gunn et al., 2000; Gunn et al., 2002; Halterman, 2013; Helman et al., 2014; Klingner \& Vaughn, 1996; Landa, 2009; Linan-Thompson et al., 2003; Montoya, 2008; Santoro et al., 2006; Solari \& Gerber, 2000; Tam et al., 2008; Vaughn, Linan-Thompson, et al., 2006; Vaughn, Mathes, et al., 2006; Wanzek \& Roberts, 2012). In each of the aforelisted studies, researchers mainly focused on analyzing measureable effects of the interventions, rather 
than analyzing the effects of the intervention in consideration of the interconnected nature of race, language, and disability. For example, Halterman (2013) used explicit instruction to teach a strategy for paraphrasing (RAP) in order to measure effects of strategy instruction on participants' reading comprehension achievement. The intervention overlooked opportunities to build on the assets of students' native language, failed to integrate schema-building activities prior to presenting textual information, and largely focused on measuring whether instruction in the RAP strategy could lead to gains on Gates Mac-Ginitie Reading Test. On the one hand, effective research isolates its focus to evaluate the relationship between an independent variable (e.g., strategy instruction) and a dependent variable (e.g., a standardized achievement test). Yet, when research includes ELs with SLD, culturally responsive researchers acknowledge and validate the cultural and linguistic assets that ELs bring to the study and also recognize the need to thoroughly describe how the intervention meets the unique needs that stem from having limited English language proficiency.

Given the evidence on effectiveness of prominent approaches for improving reading comprehension achievement in students with SLD, adjustments to the interventions reported in the current research base are needed in order to respond to culturally and linguistically diverse students with SLD. Future research can take several directions. First, very few studies to date have examined reading comprehension strategy instruction in naturally occurring inclusive classroom settings (Dole, Brown, \& Trathen, 1996; Fuchs et al., 1997). Given that research is culturally situated (Trainor \& Bal, 2014), behavior in the research setting is influenced by contextual factors (e.g., 
relationships that depict positions of power and authority between the interventionists and participants). Findings from studies that were conducted in contrived or clinical settings reflect high levels of internal validity but weak external validity. For example, a study that took place in experimenter-assigned classrooms yielded strong results when using peer-assisted learning strategies to teach reading comprehension (Sáenz et al., 2005); however, the research setting and the amount of support that classroom teachers required to implement the intervention were reported as limitations. Threats to experimental control were overcome in at least two studies that took place in naturally occurring settings and that reported gains in reading achievement (Dole et al., 1996; Fuchs et al., 1997). There is a need for more, current research to examine effectiveness of literacy interventions that are provided in authentic settings and that fully explore the contextual factors affecting the research setting.

Another direction for future research relates to the instructional materials used in reading comprehension intervention studies. Most studies that provided explicit instruction in reading comprehension strategies (e.g., summarizing, questioning, or paraphrasing) used researcher-developed passages or text published as part of a basal reading series rather than deliberately selecting culturally relevant, meaningful, and engaging text (Halterman, 2013; Santoro et al., 2006; Tam et al., 2006). Given that motivation levels affect reading comprehension performance and achievement (Guthrie, Wigfield, Metsala, \& Cox, 1999), the need exists for future intervention studies to select culturally relevant text and to engage participants' funds of knowledge (Moll, Amanti, Neff, \& González, 1992) within the context of the literacy instruction. That is, the 
context and the instruction should facilitate the use of any linguistic or cultural resource the student might have in relation to the text, the task, and the learning.

Other possible directions for future research stem from the need to investigate effectiveness of self-regulation procedures for culturally and linguistically diverse students with SLD. Evidence to support effectiveness of explicit comprehension strategy instruction with self-monitoring procedures for improving reading comprehension achievement in linguistically diverse students has yet to emerge in the literature on reading comprehension interventions. To date, no studies of comprehension strategy instruction with self-monitoring procedures have included culturally and linguistically diverse participants who were in the process of acquiring English as a second language. However, findings for monolingual students with SLD confirm the effectiveness of the practice (Jitendra et al., 2000). Given that modifications can be made to interventions that have been found effective for monolingual students, it is necessary to explore how changes to the cultural responsiveness of the interventions can impact reading comprehension performance and achievement for ELs with SLD.

Inherent to any study aimed at improving reading comprehension is the difficulty with measuring comprehension. Most commonly, researchers used standardized achievement measures or researcher-developed assessments as the sole means of determining whether gains in achievement occurred (Denton, Anthony, et al., 2004; Graves et al., 2001; Linan-Thompson et al., 2003; McElvain, 2010; Solari \& Gerber, 2008; Vaughn, Mathes, et al., 2006; Wanzek \& Roberts, 2012). Although growth can be measured (albeit sometimes with only surface-level comprehension skills) to approximate 
the effects of an intervention, the voices and thoughts of participants are hidden behind tests that may not have been validated for linguistically diverse populations, that may not have taken into account participants' funds of knowledge, thereby only awarding points to interpretations that aligned with authoritative readings. There is a need for studies to examine reading comprehension achievement from multiple perspectives through multiple measures.

Finally, only one study (Jiménez, 1997) provided instruction to ELs with SLD to improve reading comprehension using an approach that reflected cultural responsiveness. That is, participants were perceived to have valuable skills and knowledge (Klingner \& Soltero-González, 2009), the interventionist used multicultural literature, native language support, and realia during lessons (Klingner \& Soltero-González, 2009), and the interventionist reported on the nature of the relationship (i.e., insider/insider) between himself and the participants (Trainor \& Bal, 2014). Jiménez's (1997) study, however, was qualitative in nature and did not systematically measure progress with reading comprehension. Formative experiments that were built into the study provided metacognitive strategy instruction and were reported to have positive effects on participants' attitudes toward reading and on participants' perceptions of themselves as readers. The possibility remains open for a culturally responsive and systematic investigation to take place so that stronger knowledge claims can be made about the impact of comprehension strategy instruction on the reading comprehension achievement of ELs with SLD. 


\section{CHAPTER III \\ RESEARCH DESIGN}

This chapter includes the methods for the proposed research study. To begin this chapter, the design of the study, the process for selecting participants, and the research setting are discussed. Next, instructional materials and dependent measures are described. Finally, instructional procedures, testing and scoring procedures, and procedures for measuring treatment fidelity are presented.

\section{Design of the Study}

A multiple probe across participants design (Gast \& Ledford, 2010) evaluated the effects of explicit comprehension strategy instruction combined with a self-monitoring procedure on reading comprehension performance. This design relied on repeated measurement of target behaviors (i.e., application of comprehension strategies and comprehension-question answering) and controlled replication of effects across baseline and intervention conditions to establish a functional relation between the independent variable (i.e., explicit comprehension strategy instruction combined with a selfmonitoring procedure) and the dependent variables (i.e., scores on comprehension thinking strategy rubrics and percentage accuracy with answering literal and inferential comprehension questions). With this design, direct inter-subject replication of effect becomes visible through the staggered introduction of the independent variable. Given strong experimental control, three demonstrations of effect are needed at three points in 
time to document a functional relation (Horner et al., 2005).

\section{Threats to Internal Validity}

Common threats to internal validity associated with the multiple probe design jeopardize the ability to document a functional relation. I took numerous steps to reduce threats to experimental control. First, to control for the threat of attrition, I included five participants. Next, to control for inhibitive effects of testing per the guidelines of the multiple probe design, I administered performance assessments at least once per week throughout baseline and maintenance conditions and used different passages in each trial. To manage facilitative effects of testing, rather than providing participants with feedback on performance results every session, I provided feedback every fourth session in the form of a line graph that participants could visually scan to evaluate performance over time. To ensure treatment fidelity, procedural reliability data were collected in 32 to $35 \%$ of all sessions for each participant in each condition by two trained reading specialists who used observational checklists. Finally, to control for Hawthorne effects, all students in the research setting were familiar with the research setting and with me, the interventionist.

\section{Criteria for Condition Changes}

Condition changes occurred upon satisfaction of predetermined criteria related to performance on formative assessments. Upon achieving a consistent score (i.e., when $80 \%$ of the data points from instructional-level probes fell within $25 \%$ of the median), the intervention began for the first participant while the remaining four participants continued the baseline condition. After demonstrating stability in the baseline condition, 
the intervention condition began with six training sessions to introduce the comprehension strategy and the self-monitoring procedure through three modeling sessions and three guided practice sessions. The criteria to advance out of training required achieving a minimum 1-point increase over baseline scores on comprehension thinking strategy rubrics and a minimum 10-point increase over baseline percentage accuracy scores on comprehension-question answering probes.

After exiting the training phase of the intervention condition, the first participant moved into independent practice sessions with the reading comprehension strategy and the self-monitoring procedure. When the first participant attained three data points that showed an increase over guided practice sessions on: (a) accuracy with comprehensionquestion answering or (b) sophistication with applying the reading comprehension strategy, the second participant moved into the training phase of the intervention condition. This pattern continued until all participants moved from the baseline condition into the intervention condition. During independent practice sessions, when the participant completed six sessions and achieved at least three data points that maintained or increased performance during guided practice sessions, the participant exited the intervention condition. After completing the intervention, participants began the maintenance condition, during which guided reading lessons occurred daily and formative assessments were administered once per week for up to 8 weeks. Lessons in the maintenance condition followed the same procedures as lessons in the baseline condition. I administered formative assessments to evaluate application of 
comprehension strategies and accuracy with answering comprehension questions once every week during the maintenance condition.

\section{Participants}

The participants in this study were selected using purposive and convenience sampling. Given that the study aimed to identify the effectiveness of a reading comprehension intervention for English Learners (ELs) with specific learning disabilities (SLD), language status and disability status, as identified through federal criteria, were considered to identify participants. Additionally, to evaluate the effectiveness of strategies that have been used in previous research studies (e.g., Jiménez, 1997; Jitendra et al., 2000), participants needed to be able to read, write, listen, and speak in English at an intermediate proficiency level as depicted by scores on an English language proficiency assessment. Finally, based on my current teaching assignment in fifth-grade classrooms, participants were between the ages of 10 and 11 years old.

To identify potential participants, I first examined English language proficiency scores earned on the 2014 Assessing Comprehension and Communication in English State-to-State for English language learners (ACCESS; WIDA, 2010) test. I identified students in fifth grade with an overall ACCESS score between 3.0 (intermediate proficiency) and 5.0 (advanced proficiency). Next, I selected as potential participants those ELs at the intermediate proficiency levels who also had Individualized Education Programs (IEPs) with SLD as their primary special education eligibility. Finally, I verified that reading comprehension was an area of concern by examining IEP goals and accepting as potential participants those who had IEP goals focused on improving 
understanding of written language. Statements of present levels of academic achievement and functional performance were examined to identify students who were reading at least two years below grade level, on the Fountas and Pinnell Benchmark Assessment. Ultimately, six intermediate- to advanced- level ELs with IEP goals that specifically targeted reading comprehension were invited as potential participants.

To ensure that parents and the participants themselves could evaluate the merits and drawbacks of participating in this study, I provided information about the study using written and spoken language that was comprehensible (i.e., translated into the native language and free of technical or confusing terms). I indicated to parents and potential participants that I was willing to explain more about the study and to answer any questions so that they could make informed decisions about participation. Furthermore, I invited the opportunity for parents to give feedback and share any ideas they had related to the goals, procedures, and potential outcomes of the study. Providing this opportunity served as a means to assess whether the relevancy of the research problem addressed both my own line of inquiry and the participants' interests and needs. I phone calls from two Spanish-speaking parents who expressed that they valued literacy and wanted to include their children in programs that focused on improving reading.

I obtained parental informed permission and verbal assent from five participants. Then, I gathered specific information about each participant. First, I determined how and when each participant became eligible for special education services under the SLD eligibility category. Given the special education referral process in place in the research setting, commonalities emerged. Each participant became eligible to receive special 
education services under the SLD eligibility through a Response to Intervention (RTI) model and through academic testing in both English and the participant's native language (i.e., Spanish or Arabic). Next, I determined how often the participant was instructed in the general education setting. Given the program model in place in the research setting, each participant's IEP indicated that he or she remained in the general education classroom for more than $80 \%$ of the school day, where native language support was provided during content-area instruction through a transitional bilingual program model and special education services were provided using a push-in service delivery model (i.e., co-teaching). Then, I examined records of previous interventions to confirm that none of the participants had received small-group explicit instruction in using the TRACK strategy or in using self-monitoring procedures relative to reading comprehension prior to the start of the study. In addition, I collected information to provide a description of how participants' IEPs targeted deficits in reading comprehension. Each participant's reading comprehension goal aligned to the Common Core State Standards (CCSS; National Governors Association Center for Best Practices and Council of Chief State Officers, 2010) and addressed finding key ideas and details in informational text, stories, drama, or poetry. Finally, I accessed school records to obtain specific information about each participant. I determined each participant's initial special education eligibility date to determine his or her history with receiving special education services. I obtained informal survey data that presented each participant's self-reported nationality as well as each participant's language status. Information related to language status revealed whether each participant acquired his or her native language prior to learning English 
(i.e., sequential bilingualism) or whether both languages developed within the same period of time (i.e., simultaneous bilingualism). I examined each participant's home language survey to determine his or her native language. No information was available in the form of standardized test scores that could depict each participant's overall proficiency levels in his or her native language. Informal assessment data, however, revealed performance on running records with text written in the native language as well as in English. Three participants' (Miguel, Maria, and Juan) instructional reading levels in Spanish were identified to be at the Kindergarten level. One participant's (Abdul) instructional reading level in Arabic was identified to be at the preprimer level. Running records using English text indicated that three participants (Maria, Miguel, and Abdul) were reading at a second-grade level and one participant (Juan) was reading at a thirdgrade level. Additionally, I gathered each participant's level of English proficiency as indicated by his or her overall ACCESS score and reading proficiency score.

Five participants were initially included in the study. During the seventh week of the study, one participant (Mohammad) changed placements and dropped from the study. Mohammad had completed the baseline condition and three sessions in the intervention condition by the seventh week. Therefore, his data were incomplete and were not included in this study. Ultimately, results and findings are based on data collected from four EL students with SLD (Abdul, Miguel, Maria, and Juan) who participated in the study for the full 13 weeks. Background information related to each of the four participants is displayed in Table 3. 
Table 3

Participant Characteristics

\begin{tabular}{|c|c|c|c|c|c|c|}
\hline $\begin{array}{l}\text { Name } \\
\text { Gender }\end{array}$ & Age & $\begin{array}{l}\text { Initial } \\
\text { SLD } \\
\text { Eligibility }\end{array}$ & $\begin{array}{l}\text { Self- } \\
\text { reported } \\
\text { Nationality }\end{array}$ & $\begin{array}{l}\text { Language } \\
\text { Status }\end{array}$ & $\begin{array}{l}\text { Overall } \\
\text { English } \\
\text { Proficiency } \\
\text { on } 6.0 \text { Scale }\end{array}$ & $\begin{array}{l}\text { English } \\
\text { Reading } \\
\text { Proficiency } \\
\text { on } 6.0 \\
\text { Scale }\end{array}$ \\
\hline $\begin{array}{l}\text { Miguel } \\
\text { (M) }\end{array}$ & 10 & $5 / 2013$ & $\begin{array}{l}\text { Mexican } \\
\text { American }\end{array}$ & $\begin{array}{l}\text { Emerging } \\
\text { bilingual } \\
\text { Spanish/ } \\
\text { English }\end{array}$ & 4.9 & 4.5 \\
\hline $\begin{array}{l}\text { Abdul } \\
\text { (M) }\end{array}$ & 11 & $3 / 2014$ & Jordanian & $\begin{array}{l}\text { Sequential } \\
\text { bilingual } \\
\text { Arabic/ } \\
\text { English }\end{array}$ & 4.3 & 3.9 \\
\hline $\begin{array}{l}\text { Maria } \\
\text { (F) }\end{array}$ & 11 & $8 / 2011$ & $\begin{array}{l}\text { Mexican } \\
\text { American }\end{array}$ & $\begin{array}{l}\text { Emerging } \\
\text { bilingual } \\
\text { Spanish/ } \\
\text { English }\end{array}$ & 4.9 & 3.9 \\
\hline $\begin{array}{l}\text { Juan } \\
\text { (M) }\end{array}$ & 11 & $3 / 2010$ & $\begin{array}{l}\text { Mexican } \\
\text { American }\end{array}$ & $\begin{array}{l}\text { Emerging } \\
\text { bilingual } \\
\text { Spanish/ } \\
\text { English }\end{array}$ & 4.9 & 4.3 \\
\hline
\end{tabular}

\section{Setting}

The study took place in a Midwestern elementary school, with an enrollment of 643 students. The student population included a variety of nationalities (e.g., Egyptian, Jordanian, and Palestinian); the majority (75.1\%) was Caucasian. Students considered to have limited English proficiency comprised $11.4 \%$ of the school population, with the two most common native languages (other than English) being Spanish and Arabic. Students 
with disabilities comprised $15.2 \%$ of the school population, and $33 \%$ of the school population received free or reduced lunch.

Research activities took place at a kidney-shaped table at the side of a fifth-grade general education classroom during a 135-min block of time devoted to literacy instruction. I conducted intervention lessons in small groups of up to five students with and without disabilities (i.e., one participant and up to three nonparticipants per group). Each session lasted 30 to $35 \mathrm{~min}$, and met each school day for 61 days. Throughout the literacy block, four heterogeneous small groups included students who were fluent speakers of Arabic or Spanish and who exited the bilingual program; students who were low-achieving, native English speakers; and students who were average- to highachieving native speakers of English.

Using heterogeneous groups as opposed to homogeneous groups of students with similar abilities was intended as theoretically defensible practice. Previous research (Halterman, 2013; Klingner \& Vaughn, 1996; Sáenz et al., 2005) conducted with ELs with learning difficulties demonstrated improvements in reading comprehension on standardized measures of reading achievement when mixed-ability groups or pairs were used during instruction. Moreover, Gersten et al. (2001) noted that thinking aloud with a peer group is more natural than doing so with a teacher and that heterogeneous groups are more likely than homogeneous groups to promote interactive dialogue about text. In studies where researchers provided reading comprehension interventions to homogeneous groups of ELs who struggled in reading, effects on reading comprehension achievement were weak (Montoya, 2008; Wanzek \& Roberts, 2012). 
When participants were not participating in small-group instruction focused on the application of reading comprehension strategies, they rotated through and participated in three learning-center activities. This method of instruction reflects use of a coteaching model referred to as station teaching. Mixed-ability grouping is conducive to station teaching (Cook \& Friend, 1995). This model of co-teaching is premised on the idea that specific content can be taught to everyone (Cook \& Friend, 1995). Throughout the literacy block, only one station provided instruction in reading comprehension. The general education teacher led one station during which students either engaged in independent writing or one-to-one conferencing with the teacher. Another station required that students, using one-to-one computing technology tools, independently completed online literacy activities related to grammar and vocabulary. The final station was supervised by a reading teacher who provided support in general education classrooms during the literacy block. In this station, students worked in cooperative groups on research projects that connected to science or social studies curriculum, worked to improve reading fluency through a reader's theatre approach, or worked on developing and practicing test-taking strategies.

Prior to the study, I conducted an informational meeting with all teachers who provided instruction during the fifth-grade literacy block in the research setting. During the meeting, I explained and emphasized the importance of having reading comprehension instruction isolated to only one station. Throughout the study, each teacher who led a station developed his or her own lesson plans and materials for that station. Each teacher shared his or her plans with the other teachers. I evaluated shared 
lesson plans to ensure that only one teacher provided direct instruction in reading comprehension strategies throughout the literacy block.

During the reading comprehension station, I provided three phases of instruction. First, guided reading instruction was provided during baseline sessions. Next, explicit instruction in using comprehension thinking strategies with a self-monitoring procedure was provided during intervention sessions. Finally, guided reading instruction after having learned the comprehension thinking strategies and the self-monitoring procedure was provided during maintenance sessions. Movement from one phase of instruction to the next was contingent on the target participant's performance data. Four groups of students (each with one participant) rotated through the reading comprehension station each day. Group A (with Miguel, the first participant) moved from guided reading lessons in the baseline condition into explicit instruction with the comprehension strategy and self-monitoring procedure when Miguel's baseline data were stable. Group B (with the second participant, Abdul) moved into explicit instruction with the reading comprehension strategy and self-monitoring procedure when Abdul's baseline data were stable and when Miguel and Group A had progressed through at least six training sessions. Continued movement through conditions for each group was dependent upon baseline and intervention data of the participants (with Maria in Group C and Juan in Group D).

In the research setting, due to the physical layout and due to the physical abilities of all students, students rotated by moving from one station to the next. Stations remained in fixed locations within the general education classroom. To manage 
challenges associated with station teaching (e.g., high noise level), classroom management techniques were applied. For example, prior to the start of the study, expectations for movement, voice level, and activity level within each station were established, explained, visually posted, and rehearsed. Posting and rehearsing these routines ensured that the noise level in the classroom would be maintained at a comfortable level for all students and teachers. Also, to ensure that instructional pacing allowed for simultaneous transitions from one station to the next, each teacher used a visual timer and one teacher set an auditory timer to signal the closing routine and the imminent transition to the next station.

In the co-taught classroom, three licensed teachers were present during the language arts block. The general education teacher, a Caucasian female of Irish American heritage, held an elementary general education teaching license and a Master's degree in Curriculum and Instruction. At the beginning of the study, she had completed 15 years of service to the district in which the study took place, where she taught students at the intermediate grade levels. She was a native speaker of English and did not speak any languages other than English. She identified that her monolingualism could pose a barrier to effective communication with participants' families, but she expressed that collaborative efforts with bilingual staff members allowed her to feel that she was removing this barrier and keeping the lines of communication open.

A reading teacher was also present in the classroom. Her teaching experience spanned 17 years, with a 2-year hiatus between years 13 and 15 . Her service to the school district in which the study took place accounted for 14 of those years. She 
identified as a monolingual (English) White, American female of European descent. She held licensure as a general education teacher and as a reading specialist. She gained experience working with students who were native speakers of languages other than English but did not complete any formal training in teaching English as a second language or in bilingual education. She expressed a commitment to the school district's mission of "learning for all -whatever it takes." She explained that her teaching assignment involved frequent membership changes among the groups of students with whom she worked. She perceived that the short-term nature of her work precluded the formation of connections with students' parents and guardians that would position her to recognize whether she was targeting instructional goals that were valued by all stakeholders.

As the interventionist, I identified as a Caucasian, female special education teacher with ESL and Spanish bilingual teaching credentials. I held 15 years of teaching experience at the beginning of the study, with 12 years dedicated to service in the district where the study took place. My role in this school district required frequent collaboration with students and their families. My Polish American background and role in the district as an authority figure contributed to an insider/outsider relational position between participants' families and me; however, my training in cross-cultural studies and my involvement in extended school year and extended school day activities that centered on family literacy positioned me to show empathy and develop connections that provided an opportunity for establishing collaborative partnerships in the 4 months prior to start of the study. Furthermore, my proficiency in the native language of three participants' (Miguel, 
Maria, and Juan) families served to increase the quantity of collaborative conversations regarding participants' educational experiences. My lack of proficiency in Arabic prohibited my ability to communicate with the fourth participant's (Abdul) family in their native language. Abdul's family, however, stated a preference for communicating with school personnel in English rather than communicating with an Arabic-English translator. Throughout the study, I relied on assistance from an Arabic-English bilingual paraprofessional, who consulted with me on selecting resources and translating terms per Abdul's requests.

During the study, I made phone calls to connect with participants' families and I opened dialogue to ascertain that (a) families valued having a sequence of steps to follow to practice reading comprehension strategies, (b) families perceived that participants had trouble comprehending written text, and (c) families believed that reading comprehension would improve through small-group, teacher-led instruction.

\section{Instructional Materials}

\section{Instructional Technology Tools}

The study took place in a setting that provided one-to-one computing. As a result, instructional technology components were integrated into all lessons throughout the school day. To support literacy, several instructional technology tools were used as part of standard educational practice. Throughout all conditions, participants used mindmapping applications (i.e., iThoughts HD) before reading to develop a web of ideas that connected prior knowledge to the topic of the text. In every session, while reading texts or e-texts, participants used electronic or actual sticky notes to annotate text. Across 
conditions after reading, participants used a weblog (i.e., kidblog) to post insights, questions, or comments related to the text. During the intervention condition only, after completing at least three independent practice sessions, each participant used the Educreations iPad application to record himself or herself reading a self-selected 300word instructional-level passage and then developed and narrated a video by describing when, why, and how he or she applied the reading comprehension strategy by following the self-monitoring procedures.

\section{Instructional-Level Text}

Prior to the study, I selected 75 fiction and 75 nonfiction texts and e-texts written at participants' instructional reading levels, as measured through the Fountas and Pinnell Benchmark Assessment at the start of the study. I selected texts for which publishers listed the level of text difficulty according to Fountas and Pinnell's (2008) A (Kindergarten) to $\mathrm{Z}$ (eighth grade) continuum. Comprehension strategies were applicable to each of the texts used during the study.

\section{Assessment to Determine Instructional Reading Levels}

To determine an appropriate level of text difficulty, I collected data on each participant's instructional reading level in English as measured through the Fountas and Pinnell Benchmark Assessment. This assessment uses the letters A through $\mathrm{Z}$ to signify text levels that correspond to Kindergarten through eighth-grade reading levels. Administering the assessment entails conducting a running record to determine oral reading accuracy, posing scripted questions, and assessing oral and written responses to comprehension questions on a provided rubric. This assessment can be administered 
three times per school year with an elapsed time of approximately 3 months between testing sessions. Instructional reading levels were determined using Fountas and Pinnell's (2008) criteria: 90 to $94 \%$ word-reading accuracy with excellent or satisfactory comprehension or 95 to $100 \%$ word-reading accuracy with limited comprehension on text levels A through K and 95 to $97 \%$ accuracy with excellent or satisfactory comprehension or 98 to $100 \%$ word-reading accuracy with limited comprehension on text levels $\mathrm{L}$ through Z. I conducted each assessment up to 5 school days prior to the start of the baseline condition. Then, I monitored percentage accuracy with word reading as well as percentage accuracy with comprehension-question answering during the intervention condition in order to provide texts written at corresponding levels of difficulty to ensure that all lessons utilized instructional-level text. To verify progress with instructional reading levels, I readministered the Fountas and Pinnell Benchmark Assessment after each participant exited the intervention condition and began the maintenance condition.

\section{Generalization Texts}

During the generalization probes, which took place during two sessions both before and after the intervention condition, participants used text-to-speech (TTS) assistive technology (AT) in order to access information presented in on-grade-level fiction and nonfiction text. Generalization texts included culturally relevant texts or etexts written at the mid-fifth grade level (or Fountas and Pinnell level T/U). Each potential participant had an AT plan that required use of TTS to support comprehension of information contained in printed, on-grade-level text. To make the on-grade-level 
texts accessible, I either scanned texts into a Toshiba laptop in the classroom or verified that online e-texts could be read with Kurzweil 3000.

\section{Mentor Texts}

I chose mentor texts to read aloud while modeling the application of reading comprehension strategies. To select mentor texts, I first determined who the participants in the study were in terms of their native languages and cultural backgrounds and then accessed their interest inventories (completed as part of standard educational practice prior to the start of the study) to gain an understanding of participants' background knowledge and interests. After identifying potential text topics, I prioritized texts that addressed universal themes (e.g., friendship, perseverance) and used authentic language (i.e., as opposed to contrived but decodable language). Some mentor texts were written at participants' instructional reading levels. Others were written just slightly in advance of participants' instructional reading levels; however, since they were read aloud, I ensured that mentor texts were written within each participant's listening comprehension level. This was determined by consulting results from the 2014 ACCESS listening subtest and by analyzing the language in the potential mentor texts.

Mentor texts were selected from both fiction and nonfiction genres. At least one mentor text in the narrative genre portrayed a main character whose cultural and linguistic background resembled each participant while telling a story with universal themes, to which nonparticipants could relate. All mentor texts in the nonfiction genre related to topics covered in the general education science curriculum. To ensure that participants and nonparticipants could connect information in the nonfiction texts to their 
schemata, I provided direct experiences (e.g., examining a frozen glass for condensation) and used images, realia, and discussion to build background knowledge prior to reading aloud mentor texts.

\section{Prompt Cards for the Reading Comprehension Strategy}

To cue participants to use strategies to monitor for meaning while reading, the mnemonic TRACK was presented. Each letter cued participants to complete actions related to monitoring for meaning (i.e., the targeted comprehension strategy). On 3-x5inch index cards, in 18-point Helvetica font, the following words were printed: Think about what you are reading; React to the information; Ask questions; Connect; Keep track of your thinking.

\section{Self-Monitoring Procedure Cards}

Self-monitoring procedure cards are unruled 5-x 7-inch index cards with the four steps of the procedure printed in 22-point Helvetica font. The steps printed on the cards are presented in Figure 2. 


\section{Self-Monitoring Procedure Card}

$\square$ I read the paragraph.

$\square$ I used the prompt card to recall the strategy steps.

\section{$\square$ I used strategies to monitor for meaning.}

\section{$\square$ I coded the text to leave tracks of my} thinking.

Figure 2. Self-monitoring procedure card.

\section{Dependent Measures}

\section{Sophistication with Applying Reading Comprehension Strategies}

Sophistication with reading comprehension strategy application was classified based on scores achieved on the comprehension thinking strategy rubric developed by Keene (2006). Possible scores ranged from 1 (low sophistication) to 5 (high sophistication). Across conditions, I measured sophistication with applying reading comprehension strategies using the rubric for monitoring comprehension (Keene, 2006). Scores were assigned after listening to a participant read aloud from text and respond verbally to questions (e.g., what problems did you have while reading?) during a reflective conversation. Unlike the text-dependent comprehension questions, the rubric 
served as a flexible assessment tool, which was applied to texts across fiction and nonfiction genres. Additionally, it directly measured performance with comprehension strategies, which was the central focus of the study. Finally, the rubrics captured evidence from a reflective conversation, which allowed participants to articulate their thinking about a text in a way that extended beyond asking questions to see if participants could generate a predetermined answer.

\section{Accuracy with Answering Comprehension Questions}

Across conditions, each participant read at least 26 instructional-level texts and four on-grade-level texts were used during probe sessions. I alternated informative and narrative texts across sessions. For each text, I prepared five literal and five inferential questions, which I posed as a means of evaluating accuracy with comprehension-question answering. See Appendix C for literal and inferential comprehension question stems. A reading specialist crosschecked all questions before I used them in the study to assess comprehension of instructional-level text during baseline, intervention, and maintenance conditions and to assess comprehension of on-grade-level text during the generalization probes. The purposes of crosschecking were (a) to ensure that the level of difficulty of the questions was consistent across all probe texts and (b) to ensure that each question and answer aligned to a fair interpretation of the text. After posing the questions, I listened to participants' verbal responses and determined whether the initial responses were on target. I coded the accuracy of each response on a data sheet while I provided immediate verbal feedback in the form of paraphrasing participants' verbal responses. I did not immediately provide verbal feedback to indicate to the participant that his or her 
response was correct or incorrect. The purpose of paraphrasing was to check that I understood what the participant said. In cases where paraphrasing led the participant to recognize a discrepancy between his or her initial response and what was written or implied in the text, I noted the change in the participant's response, but scored only the initial responses in all sessions for all participants. For each text and each participant, I recorded the total number of on-target initial responses divided by the total number of questions posed (i.e., 10). This number was then multiplied by 100 to determine percentage accuracy. Percentage accuracy data were recorded for instructional-level text or on-grade-level text during each probe session. Graphic displays portraying performance data were shared with participants every fourth session.

\section{Reading Comprehension Achievement}

Reading comprehension achievement was measured through the Woodcock Johnson III (Woodcock, McGrew, \& Mather, 2001) passage comprehension subtest which has been validated for linguistically diverse populations and has been used for similar purposes, within a similar time frame, in: Klingner and Vaughn's (1996) study on modified reciprocal teaching; Denton, Wexler, Vaughn, and Bryan's (2008) study on collaborative strategic reading; Gunn, Biglan, Smolkowski, and Ary's (2000) and Gunn, Smolkowski, Biglan, and Black's (2002) studies to identify effects of decoding instruction on reading comprehension; and in Wanzek and Robert's (2012) study on the effects of decoding, fluency, and comprehension instruction on reading comprehension. Results from each of these studies were mixed, with some showing growth and others not reflecting a change from pretest to posttest. On the passage comprehension test of 
achievement, the initial items require the test-taker to point to the picture represented by a printed phrase. The remainder of the test presents open-ended items and requires the testtaker to read short passages and identify a missing key word that makes sense in the context of the given passage. The items become increasingly difficult by removing picture cues and by increasing the passage length, vocabulary level, and complexity of syntactic and semantic cues. For individuals aged 5 to 19 years, the passage comprehension subtest has a mean reliability score of .83 .

\section{Motivation to Read}

Two methods, a survey and an interview, were used to gather perception data on each participant's self-efficacy as a reader. First, I administered Gambrell, Palmer, Codling, and Mazzoni's (1996) Motivation to Read Profile: Reading Survey (see Appendix D -Motivation to Read Profile: Reading Survey). The survey contained 20 items, 10 of which were meant to assess each participant's self-concept as a reader and 10 items assessed participants' perceptions of the value of reading. Each item was scored on a 4-point scale, yielding an overall score of up to 80 points. The greater the overall score, the stronger the participant's motivation toward reading. Second, I conducted individual interviews to gather additional information about participants' perceptions of themselves as readers. I posed five interview questions adapted from the Motivation to Read Profile: Conversational Profile by Gambrell, Palmer, Codling, and Mazzoni (1996) (see Appendix E-Motivation to Read Profile: Reading Interview). Three questions focused on reading behaviors (e.g., "Did you read anything at home yesterday?"). One question focused on perceptions of self as a reader (e.g., "What do you need to learn to be a better 
reader?") and one question focused on general factors related to reading motivation (e.g., “Do you know of any books you would like to read?").

\section{Participants' Perceptions of the Intervention}

A 9-item Likert scale survey was administered to collect social validation data on the procedures and outcomes of the intervention (see Appendix F- Social Validation Survey). Participants' perceptions were gathered by determining the level of agreement with each of the statements. The items targeted overall level of satisfaction with the texts they read throughout the intervention condition, the instructional technology tools used throughout the study, and the comprehension strategies used during the intervention.

Surveys were generated in Google Docs so that participants could choose to activate TTS functionality via Read and Write Gold as they completed each item. Participants read or listened to each item on the survey and indicated the extent to which they agreed or disagreed with each statement, using a 6-point scale that used the following options to reflect disagreement or agreement with each statement: one, two, or two frowning faces and one, two, or three smiling faces.

Chronbach's alpha was used to measure scale reliability of the social validation instrument. The internal consistency of the survey used to measure social acceptability of the intervention was .84 . This indicates a relatively high level of internal consistency. A score of .70 is generally considered acceptable in social science research. Results were calculated by looking at participants' responses and determining the percentage of responses that fell within each category along the agreement/disagreement continuum. 


\section{Procedures}

\section{Collecting Pretest Data}

After obtaining participants' verbal assent, I gathered information on reading achievement, reading levels, and self-efficacy by administering the passage comprehension subtest of the Woodcock Johnson III, the Fountas and Pinnell Benchmark Assessment, the motivation to read survey, and the motivation to read interview in the 5 days prior to the start of the baseline condition. First, I administered the passage comprehension subtest of the Woodcock Johnson III in a separate setting (i.e., special services office) and recorded standard scores from Form A at pretest and from Form B at posttest. Next, I administered the Fountas and Pinnell Benchmark Assessment in English during the literacy block at a side table in the classroom (approximately 30 min per participant) and recorded data (percentage accuracy with word reading and level of accuracy with answering comprehension questions) on each participant's independent, instructional, and frustration reading levels. Then, I administered the motivation to read survey, which participants completed by listening to each of the 20 items read aloud through Kurzweil 3000 and by marking a response to indicate the extent to which they agreed or disagreed with each statement. Completion of the surveys was followed by individual interviews with participants. During the interviews, I posed six questions that asked about participants' perceptions of themselves as readers, and participants gave verbal responses to questions. I recorded notes on participants' responses. Then, I coded the responses to identify themes and analyzed responses to survey items to gain insight into each participant's reading self-efficacy. Data from pretests were recorded for each 
participant on a pre- and posttest data recording form (see Appendix G-Pre- and Posttest Data Recording Form).

\section{Baseline Condition}

After collecting all pretest data, the baseline condition began for all participants. In the baseline condition, I delivered reading lessons using texts on a central topic but written at each participant's instructional reading level (and each non-participant's instructional reading level). During baseline sessions, I provided instruction using English only so that the language of the intervention matched the language of classroom reading instruction. The lessons required students to read instructional-level text, to make predictions about the text, and to answer comprehension questions about the text, in keeping with participants' respective IEP goals. The baseline reading sessions did not provide explicit instruction in using or applying TRACK or the self-monitoring procedure to monitor for meaning and make sense of text. Baseline reading sessions consisted of seven specific activities that took place before, during, or after reading. Additionally, as a means of collecting generalization data, for two sessions during the baseline and two sessions during the generalization condition, participants read on-gradelevel text (read with TTS support) rather than instructional-level text. The two generalization probes allowed for participants to read on-grade-level text (with TTS support) in the fiction genre in one session and in the nonfiction genre in the second session. The purpose of having participants' read on-grade-level, or frustration level, text was to show whether performance with reading comprehension strategy instruction could transfer from instructional-level text to on-grade-level text. Participants, by virtue of 
being fifth-grade students in a general education classroom setting, were expected to attain success with grade-level proficiency standards and to demonstrate their success on high-stakes assessments that present only text that is written on grade level rather than at an instructional level.

Table 4 displays a sequence of teaching actions for all lessons used during the baseline condition of the study.

Table 4

Teaching Actions During Baseline and Maintenance Conditions

Sequence Teaching Actions

Before Ask participants to scan the text and generate predictions.

Reading

During Direct participant to use choral, echo, or silent reading to read to a Reading designated stopping point.

At the designated stopping point, ask participant to make predictions.

Have participant continue to read silently from instructional-level text.

After Pose scripted comprehension questions. Verbally paraphrase participant's

Reading responses. Do not indicate whether the response is accurate or inaccurate. Record (+) for on-target responses and (-) for off-target responses on data sheets, along with date, title of text, and genre of text.

Ask participant to talk about the text as well as to explain how he or she monitored comprehension; listen to responses. Assign a rubric score. Record rubric score and targeted strategy on data sheet.

Have participant verbally pose questions, make comments, and offer insights about what he or she read by posting to a weblog or by writing in a reader's response notebook. 
During intermittent sessions in the baseline condition, I collected data on reading comprehension performance using formative assessments. This included evaluating use of comprehension thinking strategies through reflective conversations by using comprehension thinking strategy rubrics and verbally posing literal and inferential comprehension questions to determine percentage accuracy. Data during each session were recorded on a formative assessment data recording form (see Appendix $\mathrm{H}-$ Formative Assessment Data Recording Form).

\section{Intervention Condition}

The intervention condition immediately followed the baseline condition. I delivered lessons using mentor texts and texts written at each participant's instructional reading level, as determined through the pretest Fountas and Pinnell Benchmark Assessment. I delivered explicit instruction in applying comprehension strategies. The goal of instruction was to teach participants to monitor for meaning by (a) identifying problems at the word level, sentence level, and schema level and (b) using comprehension strategies (e.g., thinking while reading, asking questions, connecting to the text, and coding the text) flexibly and appropriately. The lesson objective was for participants to apply comprehension strategies while coding text.

The mnemonic "TRACK" was used to remind students of metacognitive strategies. During the first intervention session, I provided an opportunity for participants to state preferences on wording of strategy descriptions. This allowed participants to have a voice in developing this cognitive tool. Based on preferences, "TRACK" stood for: Think about what you are reading or Think while I read; React to the information or 
React to the text; Ask questions; Connect; and Keep track of your thinking or Keep track of thinking by coding the text.

In addition, participants were taught to use a self-monitoring procedure to monitor their application of TRACK. This self-monitoring procedure was a four-step checklist:

(a) I read the paragraph; (b) I used the prompt card to recall the strategy steps (i.e., TRACK); (c) I applied the strategy to monitor for meaning; and (d) I coded the text to leave tracks of my thinking. See Table 5 for a description of the lesson progression.

Table 5

Sequence of Lessons During the Intervention Condition

\begin{tabular}{|c|c|c|c|c|c|c|c|c|c|c|c|c|}
\hline Lesson Focus & 1 & 2 & 3 & 4 & 5 & 6 & 7 & 8 & 9 & 10 & 11 & 12 \\
\hline $\begin{array}{l}\text { Modeling TRACK strategy } \\
\text { with mentor text }\end{array}$ & $\mathrm{X}$ & & & & & & & & & & & \\
\hline $\begin{array}{l}\text { Modeling TRACK + self- } \\
\text { monitoring procedures with } \\
\text { mentor text }\end{array}$ & & $\mathrm{X}$ & & & & & & & & & & \\
\hline $\begin{array}{l}\text { Guided practice in TRACK }+ \\
\text { self-monitoring procedure } \\
\text { with mentor text }\end{array}$ & & & $\mathrm{X}$ & & & & & & & & & \\
\hline $\begin{array}{l}\text { Guided practice in TRACK }+ \\
\text { self-monitoring procedure } \\
\text { with instructional-level text }\end{array}$ & & & & $X$ & $X$ & $X$ & & & & & & \\
\hline $\begin{array}{l}\text { Independent practice with } \\
\text { instructional-level text }\end{array}$ & & & & & & & $\mathrm{X}$ & $X$ & $\mathrm{X}$ & $\mathrm{X}$ & $X$ & $\mathrm{X}$ \\
\hline
\end{tabular}




\section{Training Phase}

Modeling with mentor text. During the first session in the intervention condition, I modeled using comprehension strategies to monitor for meaning while reading aloud and thinking aloud about a mentor text. During the second session in the intervention condition, I modeled using the self-monitoring procedure in addition to the comprehension strategy. For all participants, modeling took place across two 30 -min sessions. Then, the guided practice sessions began.

Guided practice lessons. During guided practice lessons, participants practiced using the TRACK comprehension strategy and the self-monitoring procedure. During the first guided practice lesson, I read aloud from a mentor text and small groups practiced using the comprehension strategy and self-monitoring procedure with feedback. I evaluated participants' reading comprehension performance as they read from instructional-level texts. Participants continued with guided practice sessions until achieving six data points in the training phase that reflected an increase over baseline scores on comprehension thinking strategy rubrics and an increase in percentage accuracy over baseline scores on comprehension tasks. The training phase comprised two modeling sessions and four guided practice sessions. Six sessions were required to cover modeling and guided practice with the TRACK comprehension strategy and the selfmonitoring procedure.

\section{Independent Practice Sessions}

After completing the training phase, independent practice sessions began. Participants worked independently to apply reading comprehension strategies and to use 
the self-monitoring procedure. Participants received feedback on use of the reading comprehension strategies (e.g., making connections, asking questions, and coding the text) and the use of the self-monitoring procedure while reading instructional-level text during each independent practice lesson. During the independent practice sessions in the intervention condition, a minimum of six data points were collected during at least six sessions. Participants remained in this phase of the condition until completing six sessions and earning three data points that showed an increase over guided practice sessions or until earning three scores of 5 on rubrics and $90 \%$ or greater on comprehension-questioning answering. Two criteria were selected so as to maintain a broad perspective on what it means to read and comprehend text. The rubric allowed reflective conversations and gave participants a chance to share their thinking, and the comprehension questions only revealed how well participants' thinking aligned to authoritative interpretations of text.

\section{Maintenance Condition}

After exiting the intervention condition, posttest data were collected and the maintenance condition began. Posttest data included administering: the Fountas and Pinnell Benchmark Assessment, the Woodcock Johnson III, the motivation to read survey, the motivation to read interview, and the social validation questionnaire. Instruction during the maintenance condition followed the same steps and sequence as instruction during the baseline condition. No direct instruction in how to apply comprehension strategies was provided. Participants, however, had access to the TRACK strategy prompt card and the self-monitoring procedure card during the maintenance condition. 
Participants, in this condition, were free to choose to use the card while reading instructional-level text. No feedback was provided on strategy use. During the maintenance condition, I assessed reading comprehension performance (on rubrics and by posing comprehension questions) at least once every week to measure comprehension of instructional-level text. In addition, twice during this condition, I assessed comprehension of on-grade-level text (fiction text in one session and nonfiction text in another) using rubrics and comprehension questions. Participants remained in the maintenance condition for 2 to 8 weeks following the intervention condition.

\section{Reliability}

\section{Procedural Reliability}

Procedural reliability data were collected in 32 to $35 \%$ of sessions for each participant, at least once per condition, through direct observational methods by two district-employed interventionists who held Master's degrees as reading specialists. The independent scorers participated in 90 min of training (broken into two 45-min sessions that occurred 6 weeks prior to the first baseline session) to build competence with evaluating procedural fidelity and with rating reading comprehension performance using the comprehension thinking strategy rubrics. The independent scorers used a checklist of procedures to rate dichotomously whether teacher-directed actions occurred or did not occur during the session (see Appendix I-Procedural Reliability Checklists for Baseline and Maintenance Conditions and Appendix J-Procedural Reliability Checklist for Intervention Lessons). The checklist displayed teacher-directed activities for baseline, intervention, generalization, and maintenance sessions. 
Data were collected from procedural reliability checklists as follows: For both raters, the total number of observed components was divided by the number of possible components and then was multiplied by 100 to determine the mean procedural reliability for each participant (Billingsley, White, \& Munson, 1980). In addition to rating whether steps of each lesson were carried out with fidelity, raters evaluated each lesson in the intervention condition using a checklist to ensure that lessons exemplified attributes of explicit instruction (e.g., stating a learning target, modeling, providing feedback, and allowing independent practice). See Appendix K for the explicit instruction checklist. Procedural fidelity data are displayed for each participant in Table 6.

Table 6

Procedural Fidelity Data

\begin{tabular}{lll}
\hline Participant & Mean Procedural Fidelity & $\begin{array}{l}\text { Fidelity to Explicit } \\
\text { Instruction }\end{array}$ \\
\hline Miguel & $100 \%$ & $100 \%$ \\
Abdul & $99 \%$ & $100 \%$ \\
Maria & $100 \%$ & $100 \%$ \\
Juan & $100 \%$ & $100 \%$ \\
\hline
\end{tabular}

\section{Interobserver Reliability}

I used the point-by-point agreement method to calculate interobserver agreement (IOA) of data on participant performance with application of reading comprehension strategies and accuracy with answering comprehension questions. IOA data were collected in 11 sessions for Miguel and Abdul, in 15 sessions for Maria, and in 10 
sessions for Juan. To calculate IOA, I divided the total number of agreements by the number of agreements plus disagreements and multiplied by 100 . This allowed me to determine mean IOA percentage (95\% for rubric scores to denote sophistication with applying reading comprehension strategies and $99 \%$ for percentage accuracy with answering comprehension questions). For each participant, IOA data are displayed in Table 7. Two participants (Maria and Juan) received relatively lower agreement scores. This was the result of a discrepancy between the raters' interpretation of text-to-self connections. Raters identified that when a student read something in the text and then shared a personal experience or stated a personal preference, he or she was making a connection to the text and monitoring for meaning. Determining whether shared experiences or stated preferences actually connected to the text caused a 1-point difference in up to $11 \%$ of scores for Maria and Juan.

Table 7

Interobserver Agreement Data

\begin{tabular}{lll}
\hline Participant & $\begin{array}{l}\text { Comprehension } \\
\text { Thinking Strategy } \\
\text { Rubrics Scores }\end{array}$ & $\begin{array}{l}\text { Accuracy with } \\
\text { Comprehension- } \\
\text { Question Answering }\end{array}$ \\
\hline Miguel & $100 \%$ & $100 \%$ \\
Abdul & $100 \%$ & $97 \%$ \\
Maria & $89 \%$ & $100 \%$ \\
Juan & $90 \%$ & $100 \%$ \\
\hline
\end{tabular}




\section{CHAPTER IV}

\section{ANALYSIS OF THE DATA}

This chapter includes a presentation of the results of the research study. The study investigated the effects of explicit reading comprehension strategy instruction with a self-monitoring procedure on the reading comprehension performance and achievement of English Learners (ELs) with Specific Learning Disabilities (SLD). A multiple probe across participants design (Gast \& Ledford, 2010) was used to evaluate the effectiveness of the reading comprehension intervention. The following research questions were posed to examine the impact of the explicit reading comprehension strategy intervention.

1. What are the effects of explicit reading comprehension strategy instruction when combined with a self-monitoring procedure on participants' application of comprehension strategies during close reading of instructional-level text?

2. What are the effects of explicit reading comprehension strategy instruction when combined with a self-monitoring procedure on participants' accuracy with answering researcher-developed literal and inferential comprehension questions after reading instructional-level text?

3. To what extent are participants able to generalize reading comprehension performance (i.e., application of comprehension strategies and accuracy with answering literal and inferential questions) while reading on-grade-level text? 
4. To what extent are participants able to maintain reading comprehension performance (i.e., application of comprehension strategies and accuracy with answering literal and inferential questions) with instructional-level text?

5. To what extent do effects of explicit reading comprehension strategy instruction with a self-monitoring procedure transfer from formative assessments to standardized measures of reading comprehension achievement?

6. How does participation in an intervention that offers explicit reading comprehension strategy instruction combined with a self-monitoring procedure affect motivation toward reading and self-concept as a reader?

7. How do participants perceive the usefulness of explicit reading comprehension strategy instruction with a self-monitoring procedure?

\section{Reading Comprehension Performance with Instructional-Level Text}

Across conditions, participants read instructional-level texts from fiction and nonfiction genres. Instructional-level texts were written at a level at which the majority of the words could be easily decoded to achieve at least $95 \%$ word-reading accuracy for level A to K text and 98\% word-reading accuracy for level $\mathrm{L}$ to $\mathrm{N}$ text. For three participants (Miguel, Abdul, and Maria) this involved reading level K to L text, and for one participant (Juan) this involved reading level $\mathrm{M}$ to $\mathrm{N}$ text, with all participants increasing their instructional reading levels during the study. To formatively assess reading comprehension of instructional-level text, participants completed two types of assessments throughout all phases in the study (i.e., baseline, training, independent practice, and maintenance). First, participants read aloud selections and engaged in 
reflective conversations with the interventionist. Direct observation of reading behaviors and information communicated through participants' self-reports were analyzed using a comprehension thinking strategy rubric. Sophistication with applying reading comprehension strategies was determined through the criteria listed on the rubric, with the highest possible score (5) corresponding to the highest level of sophistication. Second, participants verbally responded to five literal and five inferential comprehension questions. Responses to questions were assessed for accuracy, or alignment to a predetermined correct answer. Results were calculated by summing the number of correct responses, dividing by 10 (i.e., the number of questions posed) and multiplying by 100 , which yielded a percentage accuracy score. In the following sections results are presented in graphic displays in Figures 3 and 4, which portray rubric scores and percentage accuracy on answering comprehension questions after reading fiction and informational text. Additionally, Table 8 displays a breakdown of performance to communicate the mean, median, and range of scores on comprehension thinking strategy rubrics. Similarly, Table 9 shows performance results with regard to answering literal questions, separated from performance results with regard to answering inferential questions across genres.

\section{Sophistication with Applying Reading Comprehension Strategies}

The first dependent variable, sophistication with applying reading comprehension strategies, was represented through scores on the comprehension thinking strategy rubric. During the baseline condition, prior to instruction in the TRACK strategy and selfmonitoring procedure, none of the participants earned a score greater than 2 on the 5- 
point rubric. A score of 1 or 2 represented a low level of sophistication with regard to the application of strategies for monitoring for meaning while reading. Two participants (Miguel and Maria) consistently earned scores of 1, signifying little or no conscious awareness of the process of reading for meaning. Two participants (Abdul and Juan) earned mostly scores of 2 , which indicated recognition of word-level problems and identification of the need to apply a sound-it-out strategy as a solution.

After receiving instruction in using the TRACK strategy and the self-monitoring procedure, all participants' scores increased to $3 \mathrm{~s}$ and $4 \mathrm{~s}$, thereby reflecting increased sophistication in terms of the application of strategies to monitor the meaning-making process of reading. At Level 3, participants recognized sentence-level and schema-level problems, which they resolved by implementing a strategy, such as re-reading, questioning, or making connections. At Level 4, participants focused on whole-text problems and used more than one strategy to make sense of text. At a Level 5, the reader demonstrated flexible use of a variety of strategies to solve problems at the word-, sentence-, schema-, and whole-text levels: None of the participants reached this level. Descriptive measures (e.g., mean, median, and range) of each participant's data, as displayed in Table 8, and graphic representations of data for all participants (closed data points in Figure 3) indicated a change in level and trend, moving in the direction of improvement, from the baseline to the intervention (i.e., independent practice sessions) condition for all four participants.

Calculating the percentage of nonoverlapping data (PND) is one method for comparing data between adjacent conditions. According to Scruggs and Mastropieri 
(1998) an intervention can be considered effective if performance in the intervention phase (e.g., independent practice sessions of this study) does not overlap with performance in the baseline phase (e.g., guided reading lessons in the baseline condition). A three-step process is used to calculate PND: (a) count the number of intervention-phase data points that are outside the range of data in the baseline phase, (b) divide this number by the total number of data points in the intervention phase, and (c) multiply the quotient by 100 (Gast \& Ledford, 2010; Mastropieri \& Scruggs, 1998). Data comparisons from the baseline to the adjoining intervention condition revealed 100\% PND for all participants.

Miguel. During the baseline condition, Miguel's scores consistently reflected little to no conscious awareness of the process of making meaning from narrative and informational texts (a score of 1). As demonstrated in Figure 3, baseline data showed stability at a low level. After six training sessions, his data in the independent practice sessions ranged from 2 to 4 , with a median score of 3, indicating a pattern that showed a higher level over baseline performance and an accelerating trend that moved in a therapeutic direction. He earned similar scores while reading narrative text as compared to informational text. At this improved level, Miguel mainly focused on applying strategies (e.g., questioning and making connections) to solve problems at the sentence and schema level. His highest score in the intervention condition (i.e., a 4 on an informational text) did not reach the highest level of sophistication on the rubric. The effect of training was not abrupt, as the absolute level change from the baseline condition to independent practice sessions was 1 , with a relative level change of 0.5 . 
Abdul. During the baseline condition, Abdul's performance stabilized at a low level: $87 \%$ of the data points fell within a $25 \%$ range of his median score of 2 . His scores on narrative texts were consistent with his scores on informational texts. As depicted in Figure 3, Abdul's sophistication of strategy use increased to a higher level than achieved in the baseline condition. After six training sessions, data depicted an accelerating trend in the direction of improvement, with scores ranging from 3 to 4 . He earned similar scores after reading narrative and informational texts. During independent practice sessions, Abdul solved problems at the sentence-, schema-, or whole-text level by using more than one strategy throughout a passage, as guided by the TRACK mnemonic. Across conditions, the absolute level change and the relative level change were 1.

Maria. Across all baseline sessions, Maria scored 1, indicating a zero-celerating trend at a low level. Her scores after reading narrative texts matched the scores she achieved after reading informational texts. Upon completion of six training sessions, an abrupt change in performance level across conditions occurred (with an absolute and relative level change of 2). Scores were similar across genres. By solving sentence, schema, and whole-text problems with more than one strategy, she performed at a level that earned scores ranging from 3 to 4 (median score of 3 ), an increase over her baseline performance. Using the split middle method of estimation, data from the independent practice phase show a zero-celerating trend.

Juan. Across baseline sessions, Juan consistently identified and solved wordlevel problems, yielding stability at a low level (range =2-2). Across genres, no difference in scores was detectable. After completing six training sessions in which his 
scores stabilized at a higher level (median score of 3), Juan moved into the independent practice phase, and data depicted an accelerating trend in the direction of improvement (median score of 3.5). The scores he earned on fiction texts were similar to the scores he earned on informational texts. Across conditions, the absolute and relative level change was 1 , indicating that the immediacy of effect was not abrupt.

Table 8

Comprehension Thinking Strategy Rubric Scores for Instructional-Level Text

\begin{tabular}{llll}
\hline Participant & Baseline & Independent & Maintenance \\
& & Practice & \\
\hline Miguel & $\mathrm{M}=1$ & $\mathrm{M}=2.7$ & $\mathrm{M}=3$ \\
& $\mathrm{MD}=1$ & $\mathrm{MD}=3$ & $\mathrm{MD}=3$ \\
& $\mathrm{R}=1-1$ & $\mathrm{R}=2-4$ & $\mathrm{R}=2-4$ \\
Abdul & $\mathrm{M}=1.9$ & $\mathrm{M}=3.3$ & $\mathrm{M}=3.3$ \\
& $\mathrm{MD}=2$ & $\mathrm{MD}=3$ & $\mathrm{MD}=3$ \\
& $\mathrm{R}=1-2$ & $\mathrm{R}=3-4$ & $\mathrm{R}=3-4$ \\
Maria & $\mathrm{M}=1$ & $\mathrm{M}=3$ & $\mathrm{M}=3$ \\
& $\mathrm{MD}=1$ & $\mathrm{MD}=3$ & $\mathrm{MD}=3$ \\
& $\mathrm{R}=1-1$ & $\mathrm{R}=2-4$ & $\mathrm{R}=2-4$ \\
& $\mathrm{M}=2$ & $\mathrm{M}=3.5$ & $\mathrm{M}=3$ \\
& $\mathrm{MD}=2$ & $\mathrm{MD}=3.5$ & $\mathrm{MD}=3$ \\
& $\mathrm{R}=1-2$ & $\mathrm{R}=3-4$ & $\mathrm{R}=3-3$
\end{tabular}

Note. $\mathrm{M}=$ mean; $\mathrm{MD}=$ median; $\mathrm{R}=$ range 


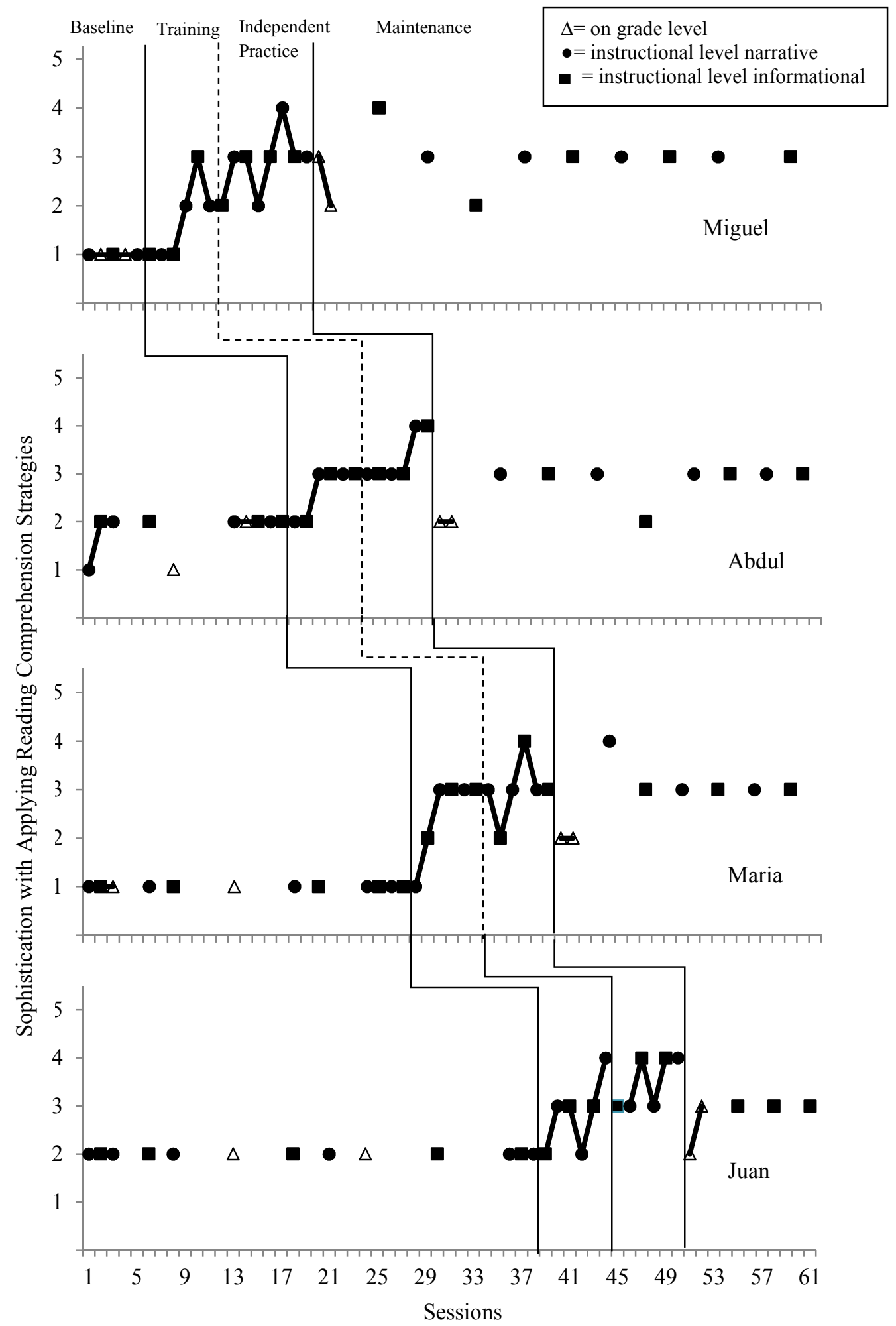

Figure 3. Sophistication with applying reading comprehension strategies across sessions. 


\section{Accuracy with Answering Reading Comprehension Questions}

The second dependent variable, accuracy with answering reading comprehension questions, was expressed as a percentage. Across conditions, five literal and five inferential questions were presented in each probe session to assess comprehension of instructional-level text. The percentage score reflects the overall score (combining literal and inferential scores) achieved during each instructional-level probe. During the baseline condition, prior to instruction in the TRACK strategy and self-monitoring procedure, all of the participants answered comprehension questions with low levels of accuracy, earning scores at or below $60 \%$; median scores ranged from 20 to $50 \%$. After receiving instruction in the TRACK strategy and the self-monitoring procedure, all participants' accuracy scores increased to a higher level; median scores ranged from 75 to $85 \%$. Graphic representations of data for all participants (closed data points in Figure 4) indicated a change in level and immediacy of effect across baseline and intervention (i.e., independent practice sessions) conditions. Data comparisons from the baseline to the adjacent intervention condition revealed 100\% PND for all participants.

Miguel. During the baseline condition, Miguel's accuracy with answering comprehension questions about instructional-level text depicted a flat trend at a low level, with no variability (range $=30-30 \%$ ). Across genres, his overall performance was the same; however, his scores for answering literal questions exceeded his scores for answering inferential questions. After completing six training sessions wherein his median accuracy was $50 \%$, he moved into the independent practice sessions. Immediacy of effect was apparent across conditions (absolute level change $=30$; relative level change 
$=40$ ). During independent practice sessions, a level shift (median score of $75 \%$ ) was accompanied by an accelerating trend in the direction of improvement, with scores ranging from 60 to $100 \%$ accuracy. During independent practice sessions, his performance was similar on fiction and informational text on both measures, and his performance with answering literal questions was again greater than his performance with answering inferential questions.

Abdul. During the baseline condition, Abdul's accuracy scores ranged from 30 to $50 \%$, with all data points falling within the stability envelope. He performed better with literal questions as compared to inferential questions; and, he earned higher scores on informational text as compared to narrative text. After completing the training phase of the intervention condition, during which his median accuracy score was $75 \%$, Abdul began independent practice sessions. An abrupt level change occurred (absolute and relative level change $=40$ ) across conditions. Responses to comprehension questions reflected an increased level of accuracy (median $=85 \%$ ) over baseline performance and a zero-celerating trend. During the intervention condition, his performance with answering literal questions was slightly stronger in comparison to performance with answering inferential questions, and his performance was slightly better with informational text as compared with narrative text.

Maria. During the baseline condition, Maria scored at low accuracy levels, with $88 \%$ of her data points falling within the range of 10 to $20 \%$ accuracy. With the exception of one outlying data point (at $40 \%$ ), baseline data showed a low, flat trend across genres. During independent practice sessions, data showed an accelerating trend 
at an increased level over baseline performance, with scores ranging from 60 to $100 \%$ accuracy. Data comparison from the baseline to the intervention condition indicates a powerful change in level (absolute and relative level change $=60$ ). Her median scores for answering literal questions about informational text were strongest, as compared to scores for answering inferential questions on texts in either genre and as compared to scores for answering literal questions on narrative text.

Juan. During the baseline condition, Juan's data depicted a low level of accuracy and a decelerating trend in a nontherapeutic direction, with scores ranging from 40 to $60 \%$ across genres. He answered literal questions with greater accuracy than he answered inferential questions. From baseline to intervention conditions, data represented a level shift, indicating immediacy of effect (absolute level change $=30$, relative level change $=20$ ). During independent practice sessions, Juan's accuracy ranged from 70 to $100 \%$, reflecting a data pattern that showed a higher level and accelerating trend in the direction of improvement. His responses to literal questions on informational texts reflected the highest level of accuracy as compared to responses to literal questions on narrative texts and responses to inferential questions on texts from both genres. 
Table 9

Accuracy with Answering Comprehension Questions on Instructional-Level Text

\begin{tabular}{|c|c|c|c|c|c|c|c|}
\hline \multicolumn{2}{|c|}{ Participant } & \multicolumn{2}{|c|}{ Baseline } & \multicolumn{2}{|c|}{ Independent Practice } & \multicolumn{2}{|c|}{ Maintenance } \\
\hline & & Literal & Inferential & Literal & Inferential & Literal & Inferential \\
\hline \multirow[t]{4}{*}{ Miguel } & $\mathrm{M}$ & $40 \%$ & $20 \%$ & $86 \%$ & $76 \%$ & $86 \%$ & $70 \%$ \\
\hline & MD & $40 \%$ & $20 \%$ & $80 \%$ & $50 \%$ & $80 \%$ & $70 \%$ \\
\hline & $\mathrm{R}$ & $40-$ & $20-20 \%$ & $60-$ & $20-100 \%$ & $80-$ & $40-100 \%$ \\
\hline & & $40 \%$ & & $100 \%$ & & $100 \%$ & \\
\hline \multirow[t]{4}{*}{ Abdul } & M & $48 \%$ & $10 \%$ & $90 \%$ & $80 \%$ & $80 \%$ & $53 \%$ \\
\hline & MD & $50 \%$ & $10 \%$ & $90 \%$ & $80 \%$ & $100 \%$ & $40 \%$ \\
\hline & $\mathrm{R}$ & $20-$ & $0-20 \%$ & $80-$ & $60-100 \%$ & $80-$ & $40-100 \%$ \\
\hline & & $80 \%$ & & $100 \%$ & & $100 \%$ & \\
\hline \multirow[t]{4}{*}{ Maria } & M & $27 \%$ & $16 \%$ & $85 \%$ & $57 \%$ & $87 \%$ & $63 \%$ \\
\hline & MD & $20 \%$ & $20 \%$ & $70 \%$ & $60 \%$ & $80 \%$ & $60 \%$ \\
\hline & $\mathrm{R}$ & $20-$ & $0-40 \%$ & $60-90 \%$ & $40-80 \%$ & $80-$ & $40-80 \%$ \\
\hline & & $40 \%$ & & & & $100 \%$ & \\
\hline \multirow[t]{4}{*}{ Juan } & M & $73 \%$ & $40 \%$ & $90 \%$ & $77 \%$ & $87 \%$ & $67 \%$ \\
\hline & MD & $80 \%$ & $40 \%$ & $90 \%$ & $80 \%$ & $80 \%$ & $60 \%$ \\
\hline & $\mathrm{R}$ & $60-$ & $0-60 \%$ & $80-$ & $60-100 \%$ & $80-$ & $60-80 \%$ \\
\hline & & $80 \%$ & & $100 \%$ & & $100 \%$ & \\
\hline
\end{tabular}

Note. $\mathrm{M}=$ mean; $\mathrm{MD}=$ median; $\mathrm{R}=$ range 


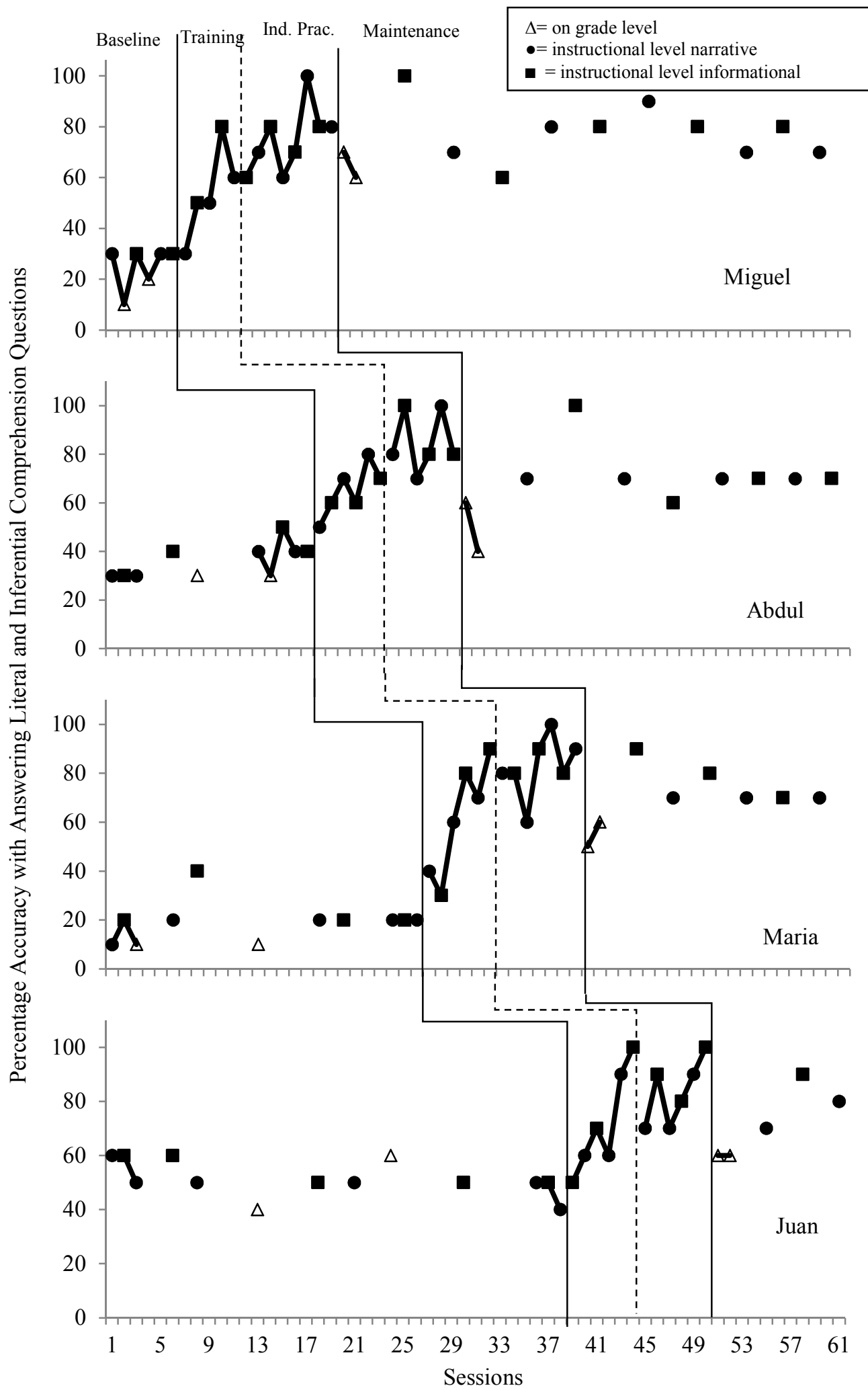

Figure 4. Accuracy with answering comprehension questions across sessions. 


\section{Reading Comprehension Performance with On-Grade-Level Text}

Generalization probes occurred during two sessions both before and after the intervention condition. During generalization sessions, participants used text-to-speech (TTS) assistive technology (AT) in order to access information presented in on-gradelevel fiction and nonfiction texts. Texts included two culturally relevant fiction texts and two informational texts related to the general science curriculum. All four generalization texts were written at the mid-fifth-grade level, equivalent to Fountas and Pinnell level T at pretest and level $U$ at posttest.

During the generalization probe in the baseline condition, two participants (Miguel and Maria) used TTS to read on-grade-level fiction text in the first generalization probe while two participants (Abdul and Juan) used TTS to read on-grade-level informational text in the first generalization probe. The same order was maintained so that Miguel and Maria also used TTS to read fiction texts first in the maintenance condition while Abdul and Juan used TTS to read informational texts first. After using TTS to read on-grade-level text, I followed the same procedures as in instructional-level probe sessions to assess reading comprehension. Participants read aloud to me (without TTS support) from the passage and engaged in a reflective conversation about comprehension thinking strategy use. I scored sophistication of application of reading comprehension strategies using a rubric. Then, I presented literal and inferential questions and evaluated accuracy of responses.

Participants' performance with on-grade-level text is depicted by open data points in Figures 3 and 4. With on-grade-level text, two participants (Miguel and Abdul) earned 
percentage accuracy scores that fell within the same range of performance as reached on instructional-level probes after exiting the intervention condition. Three participants (Miguel, Abdul, and Juan) earned rubric scores that fell within the same range of performance as reached on instructional-level probes during the baseline and maintenance conditions. Exact scores on fiction and nonfiction texts are displayed for each participant in Table 10.

Table 10

Reading Comprehension Performance with On-Grade-Level Text

\begin{tabular}{lllll}
\hline & Baseline & \multicolumn{3}{l}{ Maintenance } \\
& Fiction & Nonfiction & Fiction & Nonfiction \\
\hline Miguel & $\mathrm{S}=1$ & $\mathrm{~S}=1$ & $\mathrm{~S}=3$ & $\mathrm{~S}=2$ \\
& $\mathrm{~A}=10 \%$ & $\mathrm{~A}=20 \%$ & $\mathrm{~A}=70 \%$ & $\mathrm{~A}=60 \%$ \\
Abdul & $\mathrm{S}=2$ & $\mathrm{~S}=1$ & $\mathrm{~S}=2$ & $\mathrm{~S}=2$ \\
& $\mathrm{~A}=30 \%$ & $\mathrm{~A}=30 \%$ & $\mathrm{~A}=40 \%$ & $\mathrm{~A}=60 \%$ \\
Maria & $\mathrm{S}=1$ & $\mathrm{~S}=1$ & $\mathrm{~S}=2$ & $\mathrm{~S}=2$ \\
& $\mathrm{~A}=10 \%$ & $\mathrm{~A}=10 \%$ & $\mathrm{~A}=50 \%$ & $\mathrm{~A}=60 \%$ \\
Juan & $\mathrm{S}=2$ & $\mathrm{~S}=2$ & $\mathrm{~S}=3$ & $\mathrm{~S}=2$ \\
& $\mathrm{~A}=60 \%$ & $\mathrm{~A}=40 \%$ & $\mathrm{~A}=60 \%$ & $\mathrm{~A}=60 \%$ \\
\hline
\end{tabular}

Note. $\mathrm{S}=$ Sophistication level (rubric score); $\mathrm{A}=$ Accuracy (percentage score)

\section{Maintenance of Performance with Instructional-Level Text}

After completing at least six independent practice sessions with the TRACK strategy and self-monitoring procedure, participants moved into the maintenance 
condition, where I provided guided reading instruction, following the same procedures as in the baseline condition. Participants had access to materials from the intervention condition (e.g., the self-monitoring procedure card) but were not prompted to use these materials. Beginning 1 week from the participant's intervention-exit date, I assessed reading comprehension performance with instructional-level text at least once per week.

Performance on formative assessments during the maintenance condition is displayed for each participant in Figures 3 and 4. Descriptive measures (mean, median, and range) of data from formative assessments are displayed in Tables 8 and 9. Two weeks after exiting the intervention condition, all participants maintained performance levels above respective baseline performance on both assessments, achieving $80 \%$ accuracy on comprehension questions and earning rubric scores of 3 . The maintenance condition endured beyond 2 weeks for three participants (Miguel, Abdul, and Maria). Individual performance during the maintenance conditions is described below.

Miguel. Across an 8-week period, Miguel maintained performance at a level consistent with his performance during the intervention condition. On comprehension thinking strategy rubrics, he maintained performance to achieve an average score of 3 (median score $=3$ ). Overall, he maintained an average of $78 \%$ accuracy on answering literal and inferential comprehension questions (median score $=80 \%$ ).

Abdul. Across a 6-week period, on comprehension thinking strategy rubrics, Abdul maintained performance to achieve an average score of 3 (median score $=3$ ). Abdul maintained performance to achieve an average of $73 \%$ accuracy on answering literal and inferential comprehension questions (median score $=70 \%$ ). 
Maria. Across a 4-week period, on comprehension thinking strategy rubrics, Maria maintained performance to achieve an average score of 3.2 (median score $=3$ ). She maintained performance to achieve an average of $75 \%$ accuracy on answering literal and inferential comprehension questions (median score $=70 \%$ ). Her performance during the maintenance condition exceeded her performance during the baseline and intervention conditions in terms of percentage accuracy with literal and inferential question answering.

Juan. Across a 2-week period, on comprehension thinking strategy rubrics, Juan maintained performance to achieve an average score of 3 (median score $=3$ ). He maintained performance to achieve an average of $80 \%$ accuracy on answering literal and inferential comprehension questions (median score $=80 \%$ ).

\section{Reading Comprehension Achievement}

Up to 5 days prior to the first baseline session, all participants took a standardized achievement test, the passage comprehension subtest of the Woodcock Johnson Tests of Academic Achievement III-R (Woodcock, McGrew, \& Mather, 2001). Within 2 days of completing independent practice sessions, all participants completed the alternate form of the reading comprehension subtest. The time that lapsed between testing sessions spanned 5 weeks for Miguel, 7 weeks for Abdul, 9 weeks for Maria, and 11 weeks for

Juan. For all participants, scores at both testing sessions fell in the below-average range. Participants' scores at pre- and posttest are displayed in Table 11. 
Table 11

Reading Comprehension Achievement Scores

Participant $\quad$ Pretest Standard Score $\quad$ Posttest Standard Score

Miguel 78

79

Abdul $\quad 78$

79

Maria $\quad 76$

78

Juan $\quad 79$

79

\section{Motivation to Read}

In the 5 days prior to the first baseline session and in the 2 days following the completion of independent practice sessions, participants completed Gambrell, Palmer, Codling, and Mazzoni's (1996) Motivation to Read Profile: Reading Survey and Conversational Profile. A period of 5 to 11 weeks elapsed between the first and final administration of the survey and interview.

The conversational profile entailed using an interview script to present six questions about reading interests and behavior. The first three questions inquired about books the participants read recently, books they would like to read, and factors that influence their decisions about what to read. All participants were able to provide titles of books they had read from the school library and from language arts instruction before and after the intervention. Likewise participants were able to name titles of books they 
would like to read during the pre- and posttest interviews. Titles included books in the My Weird School series, books in the Who Would Win? series, books in the Harry Potter series, books by authors that the general education teacher had read aloud to the class (e.g., Kate Dicamillo), and nonfiction books about particular topics of interest (e.g., skate-boarding, dolphins, basketball, and killer whales). Before and after the intervention, participants expressed that their decisions about what books to read were influenced by any of three factors, including: (a) interest piqued by an appealing cover or recommendation from a friend; (b) ease of text as determined through a low number of chapters (e.g., Flat Stanley books) and/or a low number of words on each page (e.g., picture books); and (c) the point value of the book as determined through the Accelerated Reader program considered in respect to the number of points the participant needed in order to reach his or her Accelerated Reader goal.

The last three questions asked about what good readers do, how people can become better at reading, and what each participant needed to do to learn to be a better reader. Responses reflected content shifts from the pretest interview to the posttest interview. Initially, participants expressed that good readers demonstrated any of the following behaviors: reading with speed, reading with accuracy, using expression when reading aloud, and reading a high volume of chapter books (which was described as 10 or 20 books each quarter of the school year). To become better at reading, behaviors like sounding out words, reading with someone, and slowing down one's reading pace were identified by three participants (Miguel, Maria, and Juan). Abdul stated that he was not sure what a person could do to get better at reading. In reflecting on their own needs, all 
participants identified the need to improve word-reading accuracy to "get words right." Maria additionally identified the need to practice her fluency and "answer the questions [on comprehension tests] correctly." After the independent practice sessions, participants described good readers as "thinkers" and described behaviors like "asking questions, making connections, and visualizing" to explain what people could do to get better at reading. When asked to identify his or her needs relative to reading improvement, participants' responses included "practice monitoring for meaning," "continue to use schema," "keep reading more," and "use more strategies."

The survey presented 20 items (each worth four points) to assess two subcomponents of reading motivation: perceptions about the value of reading and one's concept of himself or herself as a reader. The highest possible total score on this survey was 80 points, with a maximum of 40 points for each subcomponent. According to scoring and interpretation guidelines, the closer the total score is to 80, the stronger one's motivation to read is. Participants' overall scores as well as scores for each construct (i.e., self-concept and value of reading) are presented in Table 12. 
Table 12

Motivation to Read Survey Results

\begin{tabular}{|c|c|c|c|c|c|c|}
\hline & & Pretest & & & Posttest & \\
\hline & $\begin{array}{l}\text { Self- } \\
\text { Concept }\end{array}$ & $\begin{array}{l}\text { Value of } \\
\text { Reading }\end{array}$ & $\begin{array}{l}\text { Total } \\
\text { Score }\end{array}$ & $\begin{array}{l}\text { Self- } \\
\text { Concept }\end{array}$ & $\begin{array}{l}\text { Value of } \\
\text { Reading }\end{array}$ & $\begin{array}{l}\text { Total } \\
\text { Score }\end{array}$ \\
\hline Miguel & 25 & 30 & 55 & 25 & 31 & 56 \\
\hline Abdul & 22 & 25 & 47 & 24 & 27 & 51 \\
\hline Maria & 26 & 36 & 62 & 25 & 37 & 62 \\
\hline Juan & 24 & 29 & 53 & 26 & 30 & 56 \\
\hline
\end{tabular}

\section{Participants' Perceptions of the Intervention}

All four participants completed the Likert-scale social validation surveys. The survey contained nine statements. Participants rated statements on a 6-point scale (from strongly disagree to strongly agree). Three items on the survey assessed participants' satisfaction with using technology tools. Participants expressed a neutral level of satisfaction with regard to using an idea-mapping application during prereading (mean 3.75 on a 6.0 scale) and with regard to using a weblog to share written thoughts about text while interacting with others (mean 4.25). A slightly higher level of satisfaction was expressed about using an iPad application to record a reading sample and then narrate use of comprehension strategies (mean 5.0). 
The survey was constructed to gather opinions on the texts that participants read during the intervention. Participants indicated that they enjoyed the texts they read (mean 5.0) and agreed that characters in the fiction texts resembled themselves (mean 5.0). Participants somewhat agreed (mean 4.25) that the texts used during the intervention were similar to the books that they typically read.

Finally, the participants' perceptions of the outcomes of the tools and strategies presented during the intervention were assessed. Participants agreed that the TRACK strategy helped them understand what they were reading (mean 5.0). The self-monitoring procedure was also perceived to be a helpful tool for reminding participants of strategies to use while reading (mean 5.0). Using sticky notes to code the text was perceived to be a somewhat helpful way to keeping track of thinking (mean 4.75). Table 13 includes the percentage of responses in each category for each item, as rated by all four participants. 
Table 13

Participants' Perceptions of the Intervention

\begin{tabular}{|c|c|c|c|c|c|c|}
\hline Item & $\begin{array}{l}\text { Strongly } \\
\text { Disagree }\end{array}$ & Disagree & $\begin{array}{l}\text { Somewhat } \\
\text { Disagree }\end{array}$ & $\begin{array}{l}\text { Somewhat } \\
\text { Agree }\end{array}$ & Agree & $\begin{array}{l}\text { Strongly } \\
\text { Agree }\end{array}$ \\
\hline $\begin{array}{l}\text { I liked using } \\
\text { iThoughts HD to } \\
\text { make idea maps. }\end{array}$ & $0 \%$ & $0 \%$ & $50 \%$ & $25 \%$ & $25 \%$ & $0 \%$ \\
\hline $\begin{array}{l}\text { I liked using kidblog } \\
\text { to share my thoughts } \\
\text { about texts I read. }\end{array}$ & $0 \%$ & $0 \%$ & $0 \%$ & $75 \%$ & $25 \%$ & $0 \%$ \\
\hline $\begin{array}{l}\text { I liked using } \\
\text { Educreations to } \\
\text { narrate my use of } \\
\text { reading strategies. }\end{array}$ & $0 \%$ & $0 \%$ & $0 \%$ & $25 \%$ & $50 \%$ & $25 \%$ \\
\hline $\begin{array}{l}\text { I enjoyed the books } \\
\text { that I read. }\end{array}$ & $0 \%$ & $0 \%$ & $0 \%$ & $25 \%$ & $50 \%$ & $25 \%$ \\
\hline $\begin{array}{l}\text { The characters in the } \\
\text { fiction texts that } \\
\text { were very much like } \\
\text { me and family. }\end{array}$ & $0 \%$ & $0 \%$ & $0 \%$ & $25 \%$ & $50 \%$ & $25 \%$ \\
\hline $\begin{array}{l}\text { I often read, view, or } \\
\text { listen to texts that are } \\
\text { very similar to the } \\
\text { ones I read in this } \\
\text { group. }\end{array}$ & $0 \%$ & $0 \%$ & $25 \%$ & $25 \%$ & $50 \%$ & $0 \%$ \\
\hline $\begin{array}{l}\text { Using the TRACK } \\
\text { strategy helped me } \\
\text { understand what I } \\
\text { was reading. }\end{array}$ & $0 \%$ & $0 \%$ & $0 \%$ & $0 \%$ & $100 \%$ & $0 \%$ \\
\hline $\begin{array}{l}\text { The self-monitoring } \\
\text { procedure helped me } \\
\text { remember to use } \\
\text { strategies while } \\
\text { reading. }\end{array}$ & $0 \%$ & $0 \%$ & $0 \%$ & $75 \%$ & $25 \%$ & $0 \%$ \\
\hline $\begin{array}{l}\text { Coding the text } \\
\text { helped me keep track } \\
\text { of my thinking. }\end{array}$ & $0 \%$ & $0 \%$ & $0 \%$ & $25 \%$ & $75 \%$ & $0 \%$ \\
\hline
\end{tabular}




\section{Summary}

With $100 \%$ PND and visual analysis of graphed data that depicted changes in mean, level, and trend, results indicated a functional relation between explicit reading comprehension strategy instruction with a self-monitoring procedure and participants' increased sophistication in applying reading comprehension thinking strategies as well as with participants' increased accuracy in responding to literal and inferential comprehension questions. After explicit instruction in the TRACK strategy and selfmonitoring procedure, three participants' accuracy and two participants' sophistication with applying strategies to read on-grade-level text overlapped with instructional-level performance. Standardized test scores of reading achievement slightly (but not significantly) increased or maintained at a below-average level after participants received explicit instruction in TRACK with a self-monitoring procedure. Intervention effects maintained at an improved level over baseline performance for 2 to 8 weeks. Motivation levels toward reading maintained or increased after participating in the intervention; also, participants identified that using reading comprehension strategies improved their reading. Results of the social validation survey denoted participants' satisfaction with materials and outcomes of the reading comprehension intervention. 


\section{CHAPTER V}

\section{SUMMARY, CONCLUSIONS, AND RECOMMENDATIONS}

This chapter includes a discussion based on several key findings from the present study. The main purpose of the study was to replicate and extend previous studies (Jiménez, 1997; Jitendra, Hoppes, \& Xin, 2000) on reading comprehension strategy instruction to evaluate effectiveness for English Learners (ELs) with Specific Learning Disabilities (SLD). A multiple probe across participants design was used to evaluate effects of the reading comprehension intervention for four fifth-grade participants who were ELs with SLD. Performance with reading comprehension was evaluated through comprehension thinking strategy rubrics and researcher-developed literal and inferential comprehension questions. Results indicate a functional relation between explicit reading comprehension strategy instruction and improved reading comprehension performance for all four participants. After explicit instruction, three participants' accuracy and two participants' sophistication with applying strategies to read on-grade-level text overlapped with performance on instructional-level probes. Reading achievement scores on a standardized test slightly increased for three participants and maintained for one participant but remained in the below-average range for all participants. Intervention effects maintained at an improved level over baseline for at least 2 weeks. Furthermore 
motivation levels toward reading increased or maintained at high levels, and all participants expressed that using targeted strategies improved their reading comprehension. Finally, all participants expressed satisfaction with the procedures and outcomes of the reading comprehension intervention.

In general, these results are consistent with previous research (Jiménez, 1997;

Jitendra et al., 2000; Mason, 2013) on explicit instruction in reading comprehension strategies with a self-monitoring procedure for monolingual students with SLD. Main findings and discussion points relative to ELs with SLD are discussed in the sections that follow. Then, limitations of the study, suggestions for future research, and implications for practice are discussed.

\section{Major Findings}

Main findings and discussion points are presented in an order that is organized by this study's seven research questions.

\section{Reading Comprehension Performance with Instructional-Level Text}

The first two research questions focused on investigating the effects of explicit reading comprehension strategy instruction with a self-monitoring procedure on reading comprehension performance of ELs with SLD. Reading comprehension performance was evaluated in two ways. First, comprehensions thinking strategy rubrics were used to assess sophistication with the application of strategies to monitor for meaning. Next, researcher-developed literal and inferential comprehension questions were posed to evaluate accuracy of responses. 


\section{Comprehension thinking strategy rubric scores. All participants made}

substantial improvements in the level of sophistication with which they applied comprehension thinking strategies to monitor for meaning while reading instructionallevel text during the training and independent practice phases of this study. This study was unique in using a rubric to score application of comprehension thinking strategies as measured through (a) direct observation of reading behavior and (b) reflective conversations. Previous studies evaluated use of comprehension strategies through indirect approaches or through inauthentic tasks, such as pencil-paper assessment of isolated skills (Jitendra et al., 2006) or ratings on oral retells (Hedin et al., 2011). However, in one study on ELs with learning difficulties, qualitative data (think-aloud data) were collected by Jiménez (1997) to evaluate participants'strategy application during formative experiments. Jiménez determined that prior to strategy instruction, participants struggled to monitor for meaning. Similarly in this study, prior to training, participants were unable to recognize that breakdowns in comprehension were occurring; or, participants were only aware of word-level problems and solutions (e.g., two participants knew they could attempt to sound out an unknown word). This baselineperformance level was consistent with research on reading comprehension of students with SLD (Gajria et al., 2007; Jitendra et al., 2000; Kim et al., 2012; Malone \& Mastropieri, 1992; Mason, 2013).

During the intervention condition, performance data reflected that participants gained an awareness of the process of reading for meaning and acquired strategies to monitor their comprehension and repair breakdowns at the schema-, sentence-, and 
whole-text levels, thereby earning scores of 3 and 4 on the rubric within a relatively brief period of time. An important finding is that even with a brief training period, all four participants, who experienced significant challenges with reading, learned to apply and monitor their use of reading comprehension strategies. This finding is consistent with results in the literature on integrating self-monitoring procedures into reading comprehension strategy instruction. Additionally, this finding extends the extant research base by way of its instructional focus on reading comprehension strategies other than main idea summarization (Jitendra et al., 2006; Jitendra et al., 2000; Malone \& Mastropieri, 1992).

Three reading comprehension strategies were targeted through explicit instruction in the present study: questioning, making connections, and coding text to monitor for meaning. Use of these strategies was facilitated by the TRACK procedure. During the intervention condition, all participants followed the steps in the TRACK procedure while reading by relying on both memory and reference to strategy cards. The TRACK strategy provided a structure to follow in the same way that the Think before reading, think While reading, and think After reading (TWA; Mason, Snyder, Sukhram, \& Kedem, 2006) strategy facilitated reading comprehension in prior research (Mason, 2013). Using the TRACK strategy, all participants reached a sophistication level where they applied strategies during instructional-level probes to focus on schema-level, sentence-level, and whole-text level problems (i.e., earned rubric scores of 3 or 4). With its focus on more than one comprehension strategy, TRACK facilitated the opportunity for participants to earn a rubric score of 4 or 5 . However, none of the participants earned 
scores at the highest level (i.e., 5), which would have involved demonstrating flexible and appropriate use of multiple strategies (e.g., potentially using each strategy more than once or using more than one strategy while trying to resolve a problem) while focusing on whole-text problems. During training, the interventionist modeled flexible use of the TRACK procedure, intending to avoid imposing an artificial sequence to the process of making meaning from text. In the literature on learning strategies, an artificial sequence was observed in using the POWER procedure for explanatory writing (Graham \& Harris, 2005). Nevertheless, data suggested that participants benefitted from the procedural facilitator (i.e., TRACK) by improving in their reading comprehension performance as was documented in studies on TWA (Hedin et al., 2011; Mason, 2008; Rogevich \& Perin, 2008).

Percentage accuracy with answering comprehension questions. Participants demonstrated an increase in the percentage of verbally posed, researcher-developed, literal and inferential comprehension questions answered correctly from the baseline to the intervention phase of the study. Questions were posed verbally, and participants gave verbal responses to allow broader representation of thinking to be expressed. Having participants write their responses to open-ended questions would have imposed a limit set by each participant's writing proficiency in English. During the baseline phase, all participants answered comprehension questions with $60 \%$ accuracy or less. In general, scores under $60 \%$ are considered low. During the intervention phase, all participants reached $100 \%$ accuracy, answering all of the questions in a way that aligned with a predetermined correct answer. The use of percentage accuracy and the fixed number of 
questions posed during each probe created a limit to the amount of growth that each participant could demonstrate. Ultimately, gains in comprehension demonstrated by participants in this study were more robust than those reported in studies where percentage accuracy on researcher-developed assessments was evaluated to determine the effects of reading comprehension strategy instruction with a self-monitoring procedure for monolingual students with SLD (e.g., 66\% PND in Jitendra et al., 2006).

Several factors may have contributed to robust performance in this study (e.g., use of heterogeneous, small-group instruction; use of culturally relevant text; or limitations with assessment instruments). However, one viable possibility is worthy of future exploration: The TRACK procedure differed from strategies used in previous studies in one key way. With TRACK, participants coded the text to leave tracks of their thinking. Using symbols to mark connections, questions, and interesting portions of the text made the abstract process of applying comprehension strategies (e.g., questioning, using schema, and asking questions) concrete and visible while also appearing to keep participants actively engaged in the meaning-process of reading.

\section{Reading Comprehension Performance with On-Grade-Level Text}

The third research question focused on investigating the generalizability of the reading comprehension strategies and self-monitoring procedure. Similar to the procedures used by Jitendra et al. (2000) and Malone and Mastropieri (1992) to assess generalization effects, reading comprehension strategy instruction with a self-monitoring procedure was faded and participants were given performance assessments that required reading on-grade-level text. In this study, however, after meeting criteria to exit the 
intervention phase participants used TTS to listen to the text prior to being assessed. Given that on-grade-level texts were written at a frustration level, it was expected that word-reading accuracy at less than $94 \%$ would inhibit comprehension. Listening to the text served to make its content more accessible to participants, given their difficulties

with decoding. Results showed some overlap between performance with instructionallevel text and performance with on-grade-level text on the comprehension thinking strategy rubric scores or on the percentage accuracy scores for all participants before and after the intervention. That is, participants were able to use strategies they practiced during the intervention phase of the study to monitor for meaning and make sense of ongrade-level text in order to achieve scores that were comparable to those earned on instructional-level probes in the maintenance condition.

These results allow an inference to be made about the compensatory benefit that TTS tools offered in making on-grade-level text accessible to ELs with SLD. Given that participants' performance was lower in the baseline and maintenance conditions as compared to the intervention condition, more research is needed to identify whether additional training in TRACK + the self-monitoring procedure + AT support could boost reading comprehension performance.

\section{Maintenance of Performance with Instructional-Level Text}

The fourth research question focused on maintenance of the effects of reading comprehension strategy instruction with a self-monitoring procedure. Maintenance of effects have been monitored in very few of the previous studies on reading comprehension strategy instruction with a self-monitoring procedure for monolingual 
students with SLD. Jitendra et al. (2000) found that students maintained increased reading comprehension performance levels at 6 weeks after completing independent practice sessions. Similarly, in Jitendra et al. (2006) participants showed little retention at 16 weeks but some maintenance of effects at 6 weeks. In the present study, some retention was visible at 2 weeks, 4 weeks, 6 weeks, and 8 weeks, respectively, for each of the four participants.

Lam, Shapiro, and Cole (1994) identified that maintenance of effects from a selfmonitoring procedure typically do not persist over the long term without additional reinforcement. In the present study, participants had access to the tools (i.e., strategy cards) to use at their own discretion during the maintenance phase. All four participants continued to use strategy cards during instructional-level probes in the maintenance condition for 2 weeks. Miguel, Abdul, and Maria discontinued using strategy cards during the maintenance condition but continued to make use of the text-coding symbols. Abdul, at Week 5 in the maintenance condition, was observed using the TRACK procedure (without the strategy card) while reading from a social studies textbook.

Overall, maintenance effects of explicit instruction were visible in the short term, but maintenance of effects should continue to be investigated in future studies on reading comprehension strategy instruction with self-monitoring procedures. Given the evidence in the extant literature and the results in this study, future studies should explore techniques for boosting performance over the long term when explicit instruction is faded. 


\section{Reading Comprehension Achievement}

The fifth research question investigated how effects from participation in explicit reading comprehension strategy instruction with a self-monitoring procedure would transfer from formative assessments to standardized measures of reading comprehension achievement. During this study, all participants completed the passage comprehension subtest of the Woodcock Johnson III at two points in time. This assessment presented multiple-choice questions which asked the test-taker to point to a picture in response to a phrase. These items were followed by a cloze passage, which the test-taker read in order to supply a missing word by using context clues. On this assessment, three participants achieved a slight increase in standard scores from the week preceding the baseline condition to the week following the intervention condition, and one participant maintained his score. Ultimately, all scores began and remained in the below-average range.

The strength of any inference that can be made from these results is limited. The amount of time that elapsed from one testing session to the next was brief and varied for each participant. This time period (i.e., 5 to 11 weeks) was similar to the time between tests in Denton et al., (2008) and was within the recommended test-retest window. However, results are tenuous because history poses a validity threat. In the present study, the time between testing sessions included the baseline guided reading sessions. Therefore, the extent to which guided reading instruction may have influenced outcomes on standardized achievement tests remains unknown. 


\section{Motivation to Read}

The sixth research question focused on identifying how participation in the intervention influenced participants' motivation to read. Bandura (1986) posited that motivation is the result of self-efficacy related to specific tasks. A survey and interview were administered at two points in time to evaluate participants' motivation to read. Both instruments contained items to evaluate self-efficacy and attitude toward reading. Prior to the first baseline session, participants reported a neutral to positive attitude toward reading. Participants could identify titles of books they enjoyed reading and knew of additional titles they wanted to read. Furthermore, participants were able to identify areas of strength and weakness within themselves with regard to reading comprehension and reading decoding. Given these results, motivation, prior to the intervention was strong.

After exiting the intervention, participants again evaluated their own competence with regard to reading behaviors while completing the survey and participating in the interview. All participants again communicated favorable attitudes toward reading. Survey scores did not reflect significant gains, but remained at the same moderately strong level from pre- to posttest. Participants' descriptions of themselves as readers aligned to results from performance and achievement data. The language participants used to describe their needs as readers reflected that participants internalized names of strategies (e.g., "monitoring for meaning"). This finding lends support to the claim that motivation influences the success of multiple strategy instruction (National Institute of Child Health and Human Development, 2000). 


\section{Participants' Perceptions of the Intervention}

The seventh research question aimed to assess the social validity of the intervention based on participants' perceptions. Social validation data were collected through questionnaires that investigated social acceptability of procedures and outcomes (Baer, Wolf, \& Risley, 1987) after participants exited the intervention condition. Overall, participants reported favorable impressions, or some level of agreement with regard to the usefulness or helpfulness of technology tools, texts, and instructional strategies. On the questionnaire, all participants in this study agreed that the TRACK strategy helped improve their comprehension. However, the most important measure of social acceptability of the procedures of an intervention is the direct observation of participants' preference (Hanley, 2010; Ledford, Wolery, \& Gast, 2014). During the maintenance condition, all participants were directly observed to continue using the textcoding strategy when explicit instruction faded. Moreover, all students in the general education setting made use of the strategy while reading in and out of the literacy block.

\section{Limitations and Implications for Future Research}

The main goal of the study was to identify effects of an intervention that was known to be effective for students with SLD and that was modified in its delivery to respond to participants' cultural and linguistic backgrounds. With four demonstrations of effect and 100\% PND, a functional relation between explicit reading comprehension strategy instruction and improved reading comprehension performance for all four participants was demonstrated. While the results are promising, factors that limit this research and implications for future research should be considered. 
This study used single-subject design methodology to investigate effects of a reading comprehension intervention for ELs with SLD. In keeping with the typical applications of this design, the study included a low number of participants. At the onset, five participants were selected to guard against the threat of attrition. However, due to an unanticipated change of placement, four participants completed the study. This allowed for four demonstrations of effect, reducing the threat to the study's internal validity. Given the small number of participants, the heterogeneity of ELs, and the unique characteristics associated with participants' disabilities, the study has low external validity. Therefore, the results of this study cannot be presumed effective for all ELs with SLD. Given the small likelihood of identifying large enough populations of ELs with SLD in any applied setting, investigating the research problem through group design studies poses a challenge. Therefore, additional replications of this study are needed to increase the generalizability of the study's findings.

Expectations about outcome of future replications will be shaped by several factors. First, the acronym TRACK was developed by the researcher in response to needs of the participants and their school district. Participants gave input to its development. That is, as scripted in lesson plans (see Appendix L -Scripted Intervention Lesson Plans), participants asserted their preferences between alternate choices to the phrasing of descriptions of the strategies included in TRACK. This input contributed to the cultural responsiveness of the intervention; however, the possibility is open for a different outcome to occur were input from key stakeholders (e.g., participants) to be minimized. 
Another factor that may affect the outcome of future replications of this study relates to the relationship between the interventionist and the participants. The interventionist in this study was familiar with strengths and needs of participants with regard to reading comprehension and had worked with participants for several months prior to the start of this study. This familiarity helped to control for Hawthorne effects. However, given the racial, ethnic, and linguistic differences between the interventionist and participants, the relational position is best characterized as insider/outsider. The influence of this relational position remains unknown. However, it can be expected that an insider/insider relational position or, alternatively, a lack of familiarity with participants' interests and unique learning needs would likely affect outcomes related to reading comprehension performance and achievement.

Characteristics of participants and the research setting likely influenced outcomes in a way that could be unique to this study. At the start of the study, reading motivation levels were strong and participants reported that they liked reading. In the literature, low motivation and lack of interest in reading are associated with reading difficulties experienced by students with SLD (Melekoglu, 2011). Potentially, different results would occur if participants were to begin the study with low motivation levels. There is also no way to account for how the setting (heterogeneous groupings in a general education classroom) influenced participants' performance in response to the reading comprehension intervention in this study. The setting and grouping structure provided opportunities for participants to have collaborative conversations with skilled readers and native speakers of English. In the research setting, all readers made use of TRACK and 
coded the text as they read. The intervention's social acceptability and the ancillary benefits of access to strong models of language and strategy use may be limited in a less inclusive setting.

A concern emerged with the comprehension thinking strategy rubric. It yielded quantifiable data to represent participants' metacognitive interactions with text, but scores masked some of the performance changes that were more richly captured through anecdotal records. For example, after reading about a character whose mother surprised her with ice cold watermelon, Maria made a connection to a time that her aunt prepared hot chocolate for her and her cousins. She then drew conclusions about how the characters felt. She followed this my sharing an insight on how food plays a role in bringing families close together while also connecting them to their past. Numeric scores alone failed to depict the powerful connections made, the intriguing questions posed, and the illuminating insights shared during conversations about metacognition.

Assertions about the strength of an intervention should be made with respect to its lasting effects. The participants of this study responded to explicit instruction with increases in reading comprehension performance in a brief period of time. However, maintenance data were collected for only 2 weeks, 4 weeks, 6 weeks, and 8 weeks for each of the four participants, respectively. This length of time was insufficient for determining whether effects would persist over the long term (i.e., over a semester or entire school year). Future research should evaluate long-term maintenance to identify whether participants continue to perform target behaviors (e.g., sophisticated application of reading comprehension strategies) 16 weeks or more after the intervention has been 
terminated or to identify what supports are needed (e.g., booster sessions) so that participants can maintain the target behavior over the long term.

In addition to the limits on claims that can be made about maintenance of effects in the present study, there are limits to the conclusions that can be drawn about how intervention effects transferred from instructional-level probes to on-grade-level probes. Data were collected on reading comprehension performance with on-grade-level text in two sessions before the intervention and two sessions after the intervention. Comparisons between nonadjacent conditions are not possible; consequently, within condition comparisons were made to identify effects on transfer. Within the baseline condition and again within the maintenance condition, only two data points were collected. This resulted in too few points to identify a trend. Since performance across two trials with on-grade-level probes nearly overlapped with performance across two trials with instructional-level probes, future studies should include a minimum of three generalization data points per condition.

Another consideration for future studies with regard to generalization probes relates to participants' use of TTS support while reading on-grade-level text. Participants' listening comprehension scores on the ACCESS language proficiency test and participants' familiarity with AT tools likely contributed to the compensatory benefit visible within the baseline and maintenance conditions. Future studies should ascertain the listening comprehension levels of participants and should consider participants' levels of operational competence (Cook \& Hussey, 1995) with TTS prior to integrating this AT tool into on-grade-level generalization probes. 
A final limitation of this study will likely impact all future studies on the effectiveness of explicit instruction in the application of reading comprehension strategies. Reading comprehension cannot be directly observed; therefore, it must be measured indirectly. In this study, comprehension thinking strategy rubrics and researcher-developed comprehension rubrics were used to repeatedly measure observable behaviors that signified accuracy of mental representations drawn from text and sophistication with the application of reading comprehension strategies. The rubric, an authentic assessment, facilitated reflective conversations and was juxtaposed with a traditional approach to assessment (i.e., posing comprehension questions that were scored for accuracy), which privileged one authoritative interpretation of texts. Use of two dependent measures provided contextualized information about reading comprehension performance, acknowledging the complex transactions that occur between a reader and text while reading (Rosenblatt, 1978). Future studies might also aim to reconcile the challenges associated with the construct of reading comprehension by using authentic assessments (while controlling for the threat of instrumentation) in addition to traditional assessment approaches.

In sum, participants increased reading comprehension when given explicit instruction in using the TRACK and self-monitoring procedure. On comprehension thinking strategy rubrics, none of the participants earned the highest possible score of 5 . This meant that none of the participants demonstrated flexible and appropriate use of multiple reading comprehension strategies. More research is needed to explore intervention components that could be used (e.g., additional modeling and feedback and 
other cognitive tools) to make explicit the flexible application of a variety of reading comprehension strategies, including those not addressed in this study (e.g., visualizing, inferencing, and determining importance), in order to equip ELs with SLD with sufficient literacy skills to achieve success in school and life.

\section{Implications for Practice}

The problem of low literacy achievement is relevant to practitioners who implement reading comprehension interventions to address needs of ELs who struggle with reading (including those with SLD). To date, research on effective interventions has largely been conducted with monolingual struggling readers. In this study, I aimed to address the need for more research on how to intervene with culturally and linguistically diverse students with learning difficulties. I found promising results by making specific modifications to an intervention for monolingual struggling readers. Modifications aimed to make the materials and the delivery of instruction accessible to intermediate- to advanced-proficiency-level ELs with SLD. Practitioners could benefit from evaluating current interventions and integrating any of the following components: native language support, culturally relevant text (see Appendix M- List of Culturally Relevant Texts), hands-on experiences to activate schema, and frequent opportunities for oral language development. With regard to the last component, small-group instruction with mixedability groups served to facilitate such opportunities with strong peer models.

In this study, all participants made gains relative to baseline performance on repeated measures. On a standardized test of reading achievement, participants' standard scores started and remained below average. Therefore, it is important to recognize that 
six training sessions are insufficient for closing the achievement gap. The Common Core State Standards (CCSS) require all students to grapple with a range of text complexity by reading closely to determine what the text says explicitly as well as to make logical inferences from it (National Governors Association, 2010). In this study, explicit reading comprehension strategy instruction with a self-monitoring procedure leveraged the possibility for participants to make progress toward reaching the English Language Arts CCSS. Participants began with little to no awareness of the meaning-making process of reading and with low levels of comprehension accuracy; TRACK and the self-monitoring procedure were introduced and noticeable improvements in performance occurred in a relatively brief period of time. The TRACK and self-monitoring procedure, then, may hold appeal to practitioners who are in need of an efficient means of facilitating growth in reading comprehension performance.

As a researcher, I was presented with an ethical dilemma by having to prolong the baseline period when participants' performance clearly indicated the need for intervention. Staggering the introduction of the intervention maintained experimental control; however, in classrooms, practitioners can introduce the intervention to students who are need of strategy instruction in one session rather than in four sessions that span over 2 hours of the school day. Students would benefit from not having to wait to receive needed instruction. In classrooms without additional support personnel, one teacher could manage to facilitate small-group instruction in applying TRACK and the selfmonitoring procedure in fewer than 20 min, by eliminating the repeated measurement of reading comprehension performance. This would require that the students who are not 
members of the small group would need to be engaged in a self-directed activity that can be sustained for $20 \mathrm{~min}$ (e.g., silent reading).

The acronym TRACK was developed to activate use of more than one reading strategy (i.e., using schema, questioning, and coding text to monitor for meaning). One caution must be heeded when introducing TRACK or any other learning strategy mnemonic. TRACK can impose an artificial sequence. For example, since the letter "C" follows "A" in the sequence of letters in the word "TRACK," students might default to asking questions before they make connections to the text. A similar flaw has been noted in mathematics with the acronym for the order of operations, PEMDAS or "Please excuse my dear Aunt Sally" which gives the false impression that multiplication has to come before division, when mathematically the correct answer will be calculated if the two operations are computed in a left to right sequence. Similarly, in writing, the acronym POWER (i.e., planning, organizing, writing, editing, and revising) can be interpreted to mean that no revisions can occur until after a whole text is written. However, skilled writers are vigilant and make frequent revisions throughout the writing process. To prevent this unintended artificial sequence, when introducing TRACK it is important to be clear in explaining how the letters represent strategies that can be used in any order or even simultaneously.

\section{Conclusions}

This study aimed to fill the need for reading comprehension intervention research that focused on meetings needs of culturally and linguistically diverse students who are in the process of acquiring English as a second language and who have been identified with 
SLD. The focus of the intervention aimed to equip participants with strategies to construct meaning from a variety of texts. The goal of the intervention aligned with the overall goal of reducing the reading achievement gap.

In attempting to reach toward this goal, I modified explicit reading comprehension strategy instruction in five key ways to extended previous research (Jiménez, 1997; Jitendra et al., 2000) while addressing needs of ELs with SLD. First, I perceived participants' native language and cultural backgrounds as an asset to the study. This belief was manifested through (a) use of culturally relevant texts that aligned to participants' background knowledge and experiences; (b) use of native language support to facilitate transfer from L1 to L2; and (c) use of ESL strategies to ensure that the language of instruction (i.e., English) was comprehensible to students.

Next, I formatively assessed reading comprehension through verbally posed comprehension questions as well as through reflective conversations paired with observations of reading behavior. Based on ACCESS subtest scores, participants' writing proficiency levels would have imposed false limits on the ability to communicate understanding of text. Therefore, participants verbally responded to literal and inferential comprehension questions. Moreover, reflective conversations and observations of reading behavior made visible the participants' application of targeted strategies in a way that would have been masked by solely asking comprehension questions and evaluating responses for accuracy.

Furthermore, I provided explicit strategy instruction to small, heterogeneous groups of students. This learning structure allowed for collaborative, text-centered 
conversations wherein peers without disabilities and peers who were fluent speakers of English were strong models of how to apply reading comprehension strategies to make sense of text. Additionally, I assessed the generalizability of the explicit reading comprehension strategy instruction with a self-monitoring procedure by having participants use assistive technology (e.g., text-to-speech) support to read on-grade-level text. Finally, I assessed maintenance for up to 8 weeks.

Results indicated a functional relation between explicit reading comprehension strategy instruction with a self-monitoring procedure and increases in participants' reading comprehension performance. As was the case for TWA, the TRACK procedure served as a heuristic that, according to Rosenshine's (1995) definition of a cognitive strategy, supported the development of internal procedures that enabled performance of higher-level operations. Moreover, as evidenced in Jitendra et al. (2000) and Malone and Mastropieri (1992), the self-monitoring procedure engaged participants as active learners who could regulate use of comprehension thinking strategies to monitor for meaning. Therefore, situated within the context of results found for native-English speakers with SLD, explicit instruction in using the TRACK strategy and the self-monitoring procedure played a role in equipping participants with the tools and strategies needed to approach the task of reading for meaning from the position of a skilled reader. 


\section{REFERENCES}

Abedi, J. (2002). Standardized achievement tests and English language learners: Psychometric issues. Educational Assessment, 8, 231-257. Retrieved from http://dx.doi.org/10.1207/S15326977EA080_02

Albers, C. A., \& Hoffman, A. (2012). Using flashcard drill methods and self-graphing procedures to improve the reading performance of English language learners. Journal of Applied School Psychology, 28, 367-388. doi:10.1080.15377903.2012.731365

Albus, D. A., \& Thurlow, M. L. (2007). English language learners with English language proficiency assessments: A review of state accommodation policies (Synthesis Report 66). Minneapolis, MN: University of Minnesota, National Center on Educational Outcomes.

Archer, A. L., \& Hughes, C. A. (2011). Explicit instruction: Effective and efficient teaching. What works for special needs learners. New York, NY: The Guilford Press.

Artiles, A. J., \& Ortiz, A. A. (2002). English language learners with special education needs: Identification, assessment, and instruction. McHenry, IL: Delta Systems.

Arzubiaga, A. E., Artiles, A. J., King, K. A., \& Harris-Murri, N. (2008). Beyond research on cultural minorities: Challenges and implications of research as situated cultural practice. Exceptional Children, 72, 309-327.

August, D., Francis, D. J., Hsu, H.A., \& Snow, C.E. (2006). Assessing reading comprehension in bilinguals. The Elementary School Journal, 107, 221-238.

August, D., \& Hakuta, K. (1997). Improving schooling for language-minority children: A research agenda. Washington, DC: National Academy Press.

August, D., \& Shanahan, T. (2006). Developing literacy in second-language learners: A report of the National Literacy Panel on language-minority children and youth. Mahwah, NJ: Erlbaum. 
August, D., \& Siegel, L. S. (2006). Literacy instruction for language-minority children in special education settings. In D. August \& T. Shanahan (Eds.), Developing literacy in second-language learners: A report of the National Literacy Panel on language-minority children and youth (pp. 523-554). Mahwah, NJ: Erlbaum.

Baer, D. M., Wolf, M. M., \& Risley, T. R. (1987). Some still-current dimensions of applied behavior analysis. Journal of Applied Behavior Analysis, 20, 313-327.

Bailey, A. L. (Ed.). (2007). The language demands of school: Putting academic English to the test. New Haven, CT: Yale University Press.

Bandura, A. (1986). Social foundations of thought and action: A social cognitive theory. Englewood Cliffs, NJ: Prentice-Hall.

Bartoli, J., \& Botel, M. (1988). Reading/learning disability: An ecological approach. New York, NY: Teachers College Press.

Beers, K., \& Probst, R. R. (2013). Notice and note: Strategies for close reading. Portsmouth, NH: Heinemann.

Berkeley, S., Scruggs, T. E., \& Mastropieri, M. A. (2010). Reading comprehension instruction for students with learning disabilities, 1995-2006: A metaanalysis. Remedial and Special Education, 31, 423-436.

Berninger, V. W., \& Swanson, H. L. (2014). Diagnosing and treating specific learning disabilities in relation to the brain's working memory system. In H. L. Swanson, K. R. Harris, \& S. Graham (Eds.), Handbook of learning disabilities $\left(2^{\text {nd }}\right.$ ed., pp. 307-328). New York, NY: The Guilford Press.

Berry, G., Hall, D., \& Gildroy, P. (2004). Teaching learning strategies. In B. K. Lenz \& D. D. Deschler (Eds.), Teaching content to all: Evidence-based inclusive practices in middle and secondary schools (pp. 258-278). Boston, MA: Pearson.

Biancarosa, G., \& Snow, C. E. (2006). Reading next: A vision for action and research in middle and high school literacy. Washington, DC: Alliance for Excellent Education. Retrieved from http://www.all4ed.org/files/ReadingNext.pdf

Billingsley, F. F., White, O. R., \& Munson, R. (1980). Procedural reliability: A rationale and an example. Behavioral Assessment, 2, 229-241.

Bos, C. S., \& Anders, P. L. (1992). Using interactive teaching and learning strategies to promote text comprehension and content learning for students with learning disabilities. International Journal of Disability, Development, and Education, 39, 225-238. 
Botsas, G., \& Padeliadu, S. (2003). Goal orientation and reading comprehension strategy use among students with and without reading difficulties. International Journal of Educational Research, 39(4), 477-495.

Brown, J., \& Doolittle, J. (2008). A cultural, linguistic, and ecological framework for response to intervention with English language learners. Teaching Exceptional Children, 40, 66-72.

Cain, K., \& Oakhill, J. (2006). Profiles of children with specific reading comprehension difficulties. British Journal of Educational Psychology, 76, 683-696.

Calhoon, M. B., Otaiba, S. A., Greenberg, D., King, A., \& Avalos, A. (2007). Effects of a peer-mediated program on reading skill acquisition for two-way bilingual firstgrade classrooms. Learning Disability Quarterly, 30, 169-184.

Chamot, A. U., \& O'Malley, J. M. (1996). The cognitive academic language learning approach: A model for linguistically diverse classrooms. The Elementary School Journal, 96, 259-273.

Chan, L. S. (1991). Promoting strategy generalization through self-instructional training in students with reading disabilities. Journal of Learning Disabilities, 24, 427433.

Cheng, C. K. (1998). A descriptive study of reading strategies used by Chinese ESL students from Taiwan, R. O. C. Unpublished doctoral dissertation, University of Kansas.

Cook, L., \& Friend, M. (1995). Co-teaching: Guidelines for creating effective practices. Focus on Exceptional Children, 28(3), 1-16.

Cook, A. M., \& Hussey, S. M. (1995). Assistive technology: Principles and practice. Baltimore, MD: Mosby.

Cortiella, C., \& Horowitz, S. H. (2014). The state of learning disabilities: Facts, trends and emerging issues. New York, NY: National Center for Learning Disabilities.

Council for Exceptional Children. (2014). Council for Exceptional Children standards for evidence-based practices in special education. Arlington, VA: Council for Exceptional Children.

Crosson, A. C., \& Lesaux, N. K. (2009). Revisiting assumptions about the relationship of fluent reading to comprehension: Spanish-speakers' text-reading fluency in English. Reading and Writing: A Disciplinary Journal, 23, 475-494. http://dx.doi.org/10.1007/s11145-009-9168-8. 
Cummins, J. (1979). Linguistic interdependence and educational development of bilingual children. Los Angeles, CA: National Dissemination and Assessment Center.

Damico, J. S., \& Nelson, R. L. (2010). Reading and reading impairments. In J. S. Damico, N. Müller, \& M. J. Ball (Eds.), The handbook of language and speech disorders (pp. 267-295). Chichester, UK: Wiley-Blackwell.

De La Paz, S., \& Sherman, C. K. (2013). Revising strategy instruction in inclusive settings: Effects for English learners and novice writers. Learning Disabilities Research and Practice, 28, 129-141.

Denton, C. A., Anthony, J. L., Parker, R., \& Hasbrouck, J. E. (2004). Effects of two tutoring programs on the English reading development of Spanish-English bilingual students. The Elementary School Journal, 104, 289-305.

Denton, C. A., Wexler, J., Vaughn, S., \& Bryan, D. (2008). Intervention provided to linguistically diverse middle school students with severe reading difficulties. Learning Disabilities Research and Practice, 23, 79-89.

Dexter, D. D., \& Hughes, C. A. (2011). Graphic organizers and students with learning disabilities: A meta-analysis. Learning Disability Quarterly, 34, 51-72.

Dole, J. A., Brown, K. J., \& Trathen, W. (1996). The effects of strategy instruction on the comprehension performance of at-risk students. Reading Research Quarterly, 31, 62-88.

Dole, J., Duffy, G., Roehler, L., \& Pearson, P. (1991). Moving from the old to the new: Research on reading comprehension instruction. Review of Educational Research, 6, 239-264.

Donovan, S., \& Cross, C. (2002). Minority students in special and gifted education. Washington, DC: National Academy Press.

Durkin, D. (1979). What classroom observations reveal about reading comprehension instruction. Reading Research Quarterly, 14, 481-538.

Edmonds, M. S., Vaughn, S., Wexler, J., Reutebuch, C., Cable, A., Tackett, K. K., \& Schnakenberg, J. W. (2009). A synthesis of reading interventions and effects on reading comprehension outcomes for older struggling readers. Review of Educational Research, 79(1), 262-300. doi:10.3102/0034654308325998

Fien, H., Smith, J. L. M., Baker, S. K., Chaparro, E., Baker, D. L., \& Preciado, J. A. (2010). Including English learners in a multitiered approach to early reading 
instruction and intervention. Assessment for Effective Intervention, 36, 143-157. doi: $10.1177 / 1534508410392207$

Fletcher, J. M., Lyon, G., R., Fuchs, L. S., \& Barnes, M. A. (2007). Learning disabilities: From identification to intervention. New York, NY: Guilford Press.

Freeman, Y., \& Freeman, D. (2002). Closing the achievement gap: How to reach limitedformal-schooling and long-term English language learners. Portsmouth, NH: Heinemann.

Fountas, I., \& Pinnell, G. S. (2008). Fountas and Pinnell Benchmark Assessment System. Portsmouth, NH: Heinemann.

Fuchs, D., Fuchs, L. S., Mathes, P. G., \& Simmons, D. C. (1997). Peer-assisted learning strategies: Making classrooms more responsive to diversity. American Educational Research Journal, 34, 174-186.

Gajria, M., Jitendra, A. K., Sood, S., \& Sacks, G. (2007). Improving comprehension of expository text in students with LD: A research synthesis. Journal of Learning Disabilities, 40, 210-225.

Gajria, M., \& Salvia, J. (1992). The effects of summarization instruction on text comprehension of students with learning disabilities. Exceptional Children, 58, $508-516$.

Gambrell, L. B., Palmer, B. M., Codling, R. M., \& Mazzoni, S. A. (1996). Assessing reading motivation. The Reading Teacher, 49, 518-533.

Gast, D. L., \& Ledford, J. (2010). Multiple baseline and multiple probe designs. In D. L. Gast (Ed.), Single subject research methodology in behavioral sciences (pp. 276328). New York, NY: Routledge.

Gay, G. (2000). Culturally responsive teaching: Theory, research, \& practice. New York, NY: Teachers College Press.

Genessee, F. (1978). Scholastic effects of French immersion: An overview after ten years. Interchange, 9(4), 20-29. Retrieved from: http://ink.springer.com/article/10.1007\%2FBF01189544\#page-1

Genesee, F., Lindholm-Leary, K. J., Saunders, W., \& Christian, D. (2005). Educating English language learners: A synthesis of empirical evidence. New York, NY: Cambridge University Press.

Gentile, L. (2004). The oracy instructional guide. Carlsbad, CA: Domineer Press. 
Gersten, R., Baker, S. K., Shanahan, T., Linan-Thompson, S., Collins, P., \& Scarcella, R. (2007). Effective literacy and English language instruction for English learners in the elementary grades: A practice guide (NCEE 2007-4011). Washington, DC: National Center for Education Evaluation and Regional Assistance, Institute of Education Sciences, U.S. Department of Education. Retrieved from http://ies.ed.gov/ncee/wwc/publications/practiceguides

Gersten, R., Fuchs, L. S., Williams, J. P., \& Baker, S. (2001). Teaching reading comprehension strategies to students with learning disabilities: A review of research. Review of Educational Research, 71, 279-320.

Geva, E. (2000). Issues in the assessment of reading disabilities in L2 children: Beliefs and research evidence. Dyslexia, 6, 13-28.

Geva, E., \& Massey-Garrison, A. (2013). A comparison of the language skills of ELLs and monolinguals who are poor decoders, poor comprehenders, or normal readers. Journal of Learning Disabilities, 46, 387-401. doi: $10.1177 / 0022219412466651$

Goldman-Rakic, P. S. (1992). Working memory and the mind. Scientific American, 267, 110-117.

Graham, S., \& Harris, K.R. (2005).Writing better: Effective strategies for teaching students with learning difficulties. Baltimore, MD: Brookes.

Graves, A. W. (1986). Effects of direct instruction and metacomprehension training on finding main ideas. Learning Disabilities Research, 1, 90-100.

Graves, A. W., \& Levin, J. (1989). Comparison of monitoring and mnemonic textprocessing strategies in learning disabled students. Learning Disability Quarterly, 12, 232-236.

Graves, A. W., Duesbery, N. B., Pyle, N. B., McIntosh, B. S., \& McIntosh, A. S. (2011). Two studies of tier II literacy development: Throwing sixth graders a lifeline. The Elementary School Journal, 111, 641-661.

Gunn, B., Biglan, A., Smolkowski, K., \& Ary, D. (2000). The efficacy of supplemental instruction in decoding skills for Hispanic and non-Hispanic students in early elementary school. The Journal of Special Education, 34, 90-103.

Gunn, B., Smolkowski, K., Biglan, A., \& Black, C. (2002). Supplemental instruction in decoding skills for Hispanic and non-Hispanic students in early elementary school: A follow-up. The Journal of Special Education, 36, 69-79. 
Guthrie, J. T., Wigfield, A., Metsala, J. L., \& Cox, K. E. (1999). Motivational and cognitive predictors of text comprehension and reading amount. Scientific Studies of Reading, 3, 231-257.

Halterman, T. (2013). Effects of RAP paraphrasing and semantic-mapping strategies on the reading comprehension of English learners and fully-English-proficient students with mild to moderate learning disabilities (Doctoral dissertation). Retrieved from ProQuest Dissertations and Theses database. (UMI No. 3611430).

Hanley, G. P. (2010). Toward effective and preferred programming: A case for the objective measurement of social validity with recipients of behavior-change programs. Behavior Analysis in Practice, 3, 13-21.

Harris, K. R. (1982). Cognitive-behavior modification: Application with exceptional children. Focus on Exceptional Children, 15, 1-16.

Harris, K. R., \& Graham, S. (1999). Programmatic intervention research: Illustrations from the evolution of self-regulated strategy development. Learning Disability Quarterly, 22, 251-262.

Harry, B., \& Klingner, J. K. (2012). Why are so many minority students in special education? Understanding race and disability in special education ( $2^{\text {nd }}$ ed.). New York, NY: Teachers College Press.

Harvey, S., \& Goudvis, A. (2008). Strategies that work: Teaching comprehension to enhance learning. York, ME: Stenhouse.

Hedin, L. R., Mason, L. H., \& Gaffney, J. S. (2011). Comprehension strategy instruction for two students with attention-related disabilities. Preventing School Failure, 55, 148-157. doi:10.10801045988X.2010.499393

Helman, A. L., Calhoon, M. B., \& Kern, L. (2014). Improving science vocabulary of high school English language learners with reading disabilities. Learning Disability Quarterly, 32, 1-13. doi:10.1177/0731948714539769

Hoover, J. J. (2008). Data-driven decision making in a multi-tiered model. In J. K. Klingner, J. J. Hoover, \& L. M. Baca (Eds.), Why do English language learners struggle with reading? (pp. 75-92). Thousand Oaks, CA: Corwin Press.

Horner, R. H., Carr, E. G., Halle, J., McGee, G., Odom, S., \& Wolery, M. (2005). The use of single subject research to identify evidence-based practice in special education. Exceptional Children, 71, 165-179.

Individuals with Disabilities Education Act, 20 U.S.C. $§ 1400$ et seq. (2004). 
Jiménez, R. T. (1997). The strategic reading abilities and potential of five low-literacy Latina/o readers in middle school. Reading Research Quarterly, 32, 224-243.

Jitendra, A. K., Burgess, C., \& Gajria, M. (2011). Cognitive strategy instruction for improving expository text comprehension of students with learning disabilities: The quality of evidence. Exceptional Children, 77, 135-159.

Jitendra, A. K., Cole, C. L., Hoppes, M. K., \& Wilson, B. (1998). Effects of a direct instruction main idea summarization program and self-monitoring on reading comprehension of middle school students with learning disabilities. Reading \& Writing Quarterly, 14, 379-396.

Jitendra, A. K., Hoppes, M., \& Xin, Y. P. (2000). Enhancing main idea comprehension for students with learning problems: The role of a summarization strategy and self-monitoring instruction. Journal of Special Education, 34, 127-139.

Kalyanpur, M., \& Harry, B. (2012). Cultural reciprocity in special education: Building family-professional partnerships. Baltimore, MD: Brookes.

Kavale, K.A., \& Forness, S.R. (1996). Social skill deficits and learning disabilities: A meta-analysis. Journal of Learning Disabilities, 29, 226-227.

Keene, E. O. (2006). Assessing comprehension thinking strategies. Huntington Beach, CA: Shell Education.

Kim, W., Linan-Thompson, S., \& Misquitta, R. (2012). Critical factors in reading comprehension instruction for students with learning disabilities: A research synthesis. Learning Disabilities Research \& Practice, 27, 66-78.

Kim, A., Vaughn, S., Klingner, J. K., Woodruff, A. L., Reutebuch, C. K., \& Kouzekanani, K. (2006). Improving the reading comprehension of middle school students with disabilities through computer-assisted collaborative strategic reading. Remedial and Special Education, 27, 235-249.

Kim, A., Vaughn, S., Wanzek, J., \& Wei, S. (2004). Graphic organizers and their effects on the reading comprehension of students with LD: A synthesis of research. Journal of Learning Disabilities, 37, 105-118.

Klingner, J. K., Artiles A. J., \& Méndez Barletta, L. (2006). English language learners who struggle with reading. Language acquisition or learning disabilities? Journal of Learning Disabilities, 39, 108-128. 
Klingner, J. K., Boardman, A., G., Eppolito, A. M., \& Schonewise, E. A. (2012). Supporting adolescent English language learners' reading in the content areas. Learning Disabilities - A Contemporary Journal, 10, 35-64.

Klingner, J. K., Boelé, A., Linan-Thompson, S., \& Rodriguez, D. (2014). Essential components of special education for English language learners with learning disabilities: Position statement of the Division for Learning Disabilities of the Council for Exceptional Children. Learning Disabilities Research and Practice, 29, 93-96.

Klingner, J. K., \& Edwards, P. (2006). Cultural considerations with response to intervention models. Reading Research Quarterly, 41, 108-117.

Klingner, J. K., \& Eppolito, A. M. (2014). English language learners: Differentiating between language acquisition and learning disabilities. Alexandria, VA: Council for Exceptional Children.

Klingner, J. K., \& Geisler, D. (2008). Helping classroom reading teachers distinguish between language acquisition and learning disabilities. In J. K. Klingner, J. J. Hoover, \& L. M. Baca, (Eds.), Why do English language learners struggle with reading? (pp. 57-74). Thousand Oaks, CA: Corwin Press.

Klingner, J. K., \& Soltero-Gonzalez, L. (2009). Culturally and linguistically responsive literacy instruction for English language learners with learning disabilities. Multiple Voices for Ethnically Diverse Exceptional Learners, 12(1), 4-20.

Klingner, J. K., \& Vaughn, S. (1996). Reciprocal teaching of reading comprehension strategies for students with learning disabilities who use English as a second language. The Elementary School Journal, 96, 275-293.

Klingner, J. K., Vaughn, S., Arguelles, M. E., Hughes, M. T., \& Leftwich, S. A. (2004). Collaborative strategic reading: "Real-world" lessons from classroom teachers. Remedial and Special Education, 25, 291-302.

Klingner, J. K., Vaughn, S., \& Boardman, A. (2007). Teaching reading comprehension to students with learning difficulties. New York, NY: The Guilford Press.

Klingner, J. K., Vaughn, S., \& Schumm, J. (1998). Collaborative strategic reading during social studies in heterogeneous fourth-grade classrooms. Elementary School Journal, 99, 3-22.

Krashen, S. (1981). Second language acquisition and second language learning. Oxford: Pergamon Press. 
Krashen, S. (2009). Does intensive decoding instruction contribute to reading comprehension? Knowledge Quest, 37, 72-74.

Krashen, S. D., \& Terrell, T. D. (1983). The natural approach: Language acquisition in the classroom. San Francisco, CA: The Alemany Press.

Kress, J. (2008). The ESL/ELL teacher's book of lists. San Francisco, CA: Jossey-Bass.

Ledford, J., Wolery, M., \& Gast, D. L. (2014). Controversial and critical issues in single case research. In D. L. Gast \& J. R. Ledford (Eds.), Single case research methodology: Applications in special education and behavioral sciences (pp. 377396). New York, NY: Routledge.

Lado, R. (1964). Language teaching: A scientific approach. New York, NY: McGraw Hill.

Lam, A. L., Cole, C. L., \& Shapiro, E. (1994). Relative effects of self-monitoring on-task behavior, academic accuracy, and disruptive behavior in students with behavior disorders. School Psychology Review, 23, 44-58.

Landa, K. G. (2009). Effects of repeated readings on reading abilities of English language learners with specific learning disabilities (Doctoral dissertation). Retrieved from ProQuest Dissertations and Theses database. (UMI No. 339505).

Landson-Billings, G. (1994). The dreamkeepers: Successful teachers for AfricanAmerican children. San Francisco, CA: Jossey-Bass.

Lerner, J. W. (2003). Learning disabilities: Theory, diagnosis, and teaching strategies ( $9^{\text {th }}$ ed.). Boston, MA: Houghton Mifflin Co.

Lesaux, N. K., \& Harris, J. R. (2013). Linguistically diverse students' reading difficulties: Implications for models of learning disabilities identification and effective instruction. In H. L. Swanson, K. R. Harris, \& S. Graham (Eds.), Handbook of learning disabilities ( ${ }^{\text {nd }}$ ed., pp. 69-84). New York, NY: The Guilford Press.

Linan-Thompson, S., \& Vaughn, S. (2007). Research-based methods of reading instruction for English language learners grades K-4. Alexandria, VA: Association for Supervision and Curriculum Development.

Linan-Thompson, S., Vaughn, S., Hickman-Davis, P., \& Kouzekanani, K. (2003). Effectiveness of supplemental reading instruction for second-grade English language learners with reading difficulties. The Elementary School Journal, 103, 221-238. 
Malone, L., \& Mastropieri, M. (1992). Reading comprehension instruction: Summarization and self-monitoring training for students with learning disabilities. Exceptional Children, 58, 270-279.

Mason, L. H. (2013). Teaching students who struggle with learning to think before, while, and after reading: Effects of self-regulated strategy development instruction. Reading and Writing Quarterly, 29, 124-144.

Mason, L. R. (2004). Explicit self-regulated strategy development versus reciprocal questioning: Effects on expository reading comprehension among struggling readers. Journal of Educational Psychology, 96, 283-296.

Mason, L. H., Snyder, K. H., Sukhram, D. P., \& Kedem, Y. (2006). TWA + PLANS strategies for expository reading and writing: Effects for nine fourth-grade students. Exceptional Children, 73, 69-89.

Mastropieri, M. A., \& Scruggs, T. E. (2002). Effective instruction for special education ( ${ }^{\text {rd }}$ ed.). Austin, TX: Pro-Ed.

Mastropieri, M. A., Scruggs, T. E., Bakken, J. P., \& Whedon, C. (1996). Reading comprehension: A synthesis of research in learning disabilities. In T. E. Scruggs \& M. A. Mastropieri (Eds.), Advances in learning and behavioral disorders (Vol. 10B, pp. 201-227). New York, NY: Elsevier Science/JAI Press.

McElvain, C. M. (2010). Transactional literature circles and the reading comprehension of English learners in the mainstream classroom. Journal of Research in Reading, 33, 178-205. doi:10.1111/j.1467-9817.2009.0143.x

Melekoglu, M. A. (2011). Impact of motivation to read on reading gains for struggling readers with and without learning disabilities. Learning Disability Quarterly, 34, 248-261.

Miranda A., Villaescusa, I., \& Vidal-Abarca, E. (1997). Is attribution retraining necessary? Use of self-regulation procedures for enhancing the reading comprehension strategies of children with learning disabilities. Journal of Learning Disabilities, 30, 503-512.

Moll, L. C., Amanti, C., Neff, D., \& Gonzalez, N. (1992). Funds of knowledge for teaching: Using a qualitative approach to connect homes and classrooms. Theory into Practice, 31, 132-141.

Montoya, L. P. A. C. (2008). Effects of guided reading (teacher supported) comprehension scores for primary students who are learning disabled English 
language learners (Doctoral dissertation). Retrieved from ProQuest Dissertations and Theses database. (UMI No. 3341899).

Moore, B. A., \& Klingner, J. K. (2012). Considering the needs of English language learner populations: An examination of the population validity of reading intervention research. Journal of Learning Disabilities, 45, 1-18. doi: $10.1177 / 0022219412466702$

National Center for Education Statistics. (2013). The condition of education 2013. (NCES 2013-037), English language learners. Washington, DC: U.S. Department of Education.

National Clearinghouse for English Language Acquisition. (2011, February). The growing numbers of English learner students, 1998/99-2008/09. Retrieved from http://www.ncela.us/files/uploads/9/growingLEP_0809.pdf

National Governors Association Center for Best Practices and Council of Chief State School Officers. (2010). Common Core State Standards for English language arts and literacy in history/social studies, science, and technical subjects. Washington, D.C.: Authors.

National Institute of Child Health and Human Development. (2000). Report of the National Reading Panel. Teaching children to read: An evidence-based assessment of the scientific research literature on reading and its implications for reading instruction (NIH Publication No. 00- 4769). Washington, DC: U.S. Government Printing Office.

No Child Left Behind (NCLB) Act of 2001, Pub. L. No. 107-110, § 1001, Stat. 1425 (2002).

O'Connor, R. E., White, A., \& Swanson, H. L. (2007). Repeated reading versus continuous reading: Influences on reading fluency and comprehension. Exceptional Children, 74, 31- 46.

Ortiz, A. (2001). English language learners with special needs: Effective instructional strategies. LD Online. Retrieved from http://www.ldonline.org/article/5622? theme=print

Ortiz, A. A., Wilkinson, C. Y., Robertson-Courtney, P., \& Kushner, M. I. (2006). Considerations in implementing intervention assistance teams to support English language learners. Remedial and Special Education, 27, 53-63. doi: $10.1177 / 07419325060270010601$ 
Ortiz, A. A., \& Yates, J. R. (2008). Enhancing scientifically-based research for culturally and linguistically diverse learners. Multiple Voices for Ethnically Diverse Exceptional Learners, 11, 13-23.

Orosco, M. J., \& Klingner, J. K. (2010). One school's implementation of RTI with English language learners: Referring into RTI. Journal of Learning Disabilities, 43, 269-288.

Oswald, D. P., Coutinho, M. J., Best, A. M., \& Singh, N. N. (1999). Ethnic representation in special education: The influence of school related economic and demographic variables. Journal of Special Education, 32, 194-206.

Paradis, J., Genesee, F., \& Crago, M. B. (2011). Dual language development and disorders: A handbook on bilingualism and second language learning (2nd ed.). Baltimore, MD: Brookes.

Paris, S. G., Wasik, B. A., \& Turner, J. C. (1991). The development of strategic readers. In R. Barr, M. L. Kamil, P. B. Mosenthal, \& P. D., Pearson (Eds.), Handbook of reading research (Vol. 2, pp. 609-640). New York, NY: Longman.

Peregoy, S. F., \& Boyle, O. F. (2008). Reading, writing, and learning in ESL: A resource book for K-12 English learners. Boston, MA: Pearson Education, Inc.

Perfetti, C. A. (1985). Some reasons to save the grapheme and the phoneme. Commentary in Brain and Behavior Sciences, 8(4), 1-2.

Proctor, C. P., Dalton, B., \& Grisham, D. L. (2007). Scaffolding English language learners and struggling readers in a universal literacy environment with embedded strategy instruction and vocabulary support. Journal of Literacy Research, 39, 7193. doi:10.1080/10862960709336758

Rinaldi, C. (October, 2011). Keeping special education in proportion: Experts say improvements in school instructional cultures can keep struggling minority kids out of special education, Education Week. Retrieved from:

http://www.edweek.org/tsb/articles/2011/10/13/01disproportion.h05.html?qs=clau dia+rinaldi

Roberts, G., Torgesen, J. K., Boardman, A., \& Scammacca, N. (2008). Evidence-based strategies for reading instruction of older students with learning disabilities. Learning Disabilities Research \& Practice, 23, 63-69.

Rogevich, M. E., \& Perin, D. (2008). Effects on science summarization of a reading comprehension intervention for adolescents with behavior and attention disorders. Exceptional Children, 74, 135-154. 
Rosenshine, B. (1995). Advances in research on instruction. Journal of Educational Research, 88, 262-268.

Rosenblatt, L. M. (1978). The reader, the text, the poem: The transactional theory of literary work. Carbondale, IL: Southern Illinois University Press.

Rueda, R., MacGillivray, L., Monzó, L., \& Arzubiaga, A. (2001). Engaged reading: A multi-level approach to considering sociocultural factors with diverse learners. In D. McInerny \& S. VanEtten (Eds.), Research on sociocultural influences on motivation and learning. (pp. 233-264). Charlotte, NC: Information Age Publishing.

Ryan, E. B. (1981). Identifying and remediating failures on reading comprehension: Toward an instructional approach for poor comprehenders. In G. E. Mackinnon \& T. G. Waller (Eds.), Reading research: Advances in theory and practice (Vol. 3, pp. 223-261). New York, NY: Academic Press.

Sáenz, L. M., Fuchs, L. S., \& Fuchs, D. (2005). Peer-assisted learning strategies for English language learners with learning disabilities. Exceptional Children, 71, 231-247.

Samson, J. F., \& Lesaux, N. K. (2009). Language-minority learners in special education: Rates and predictors of identification for services. Journal of Learning Disabilities, 42, 148-162.

http://dx.doi.org.proxy.lib.ilstu.edu/10.1177/0022219408326221

Santoro, L. E., Jitendra, A. K., Starosta, K., \& Sacks, G. (2006). Reading well with "Read Well": Enhancing the reading performance of English language learners. Remedial and Special Education, 27, 105-115.

Schiefele, U. (1996). Topic interest, text representation, and quality of experience. Contemporary Educational Psychology, 21, 3-18.

Schumaker, J. B., Denton, P. H., \& Deshler, D. D. (1984). The paraphrasing strategy. Lawrence, KS: The University of Kansas.

Scruggs, T., \& Mastropieri, M.A. (1998) Summarizing single-subject research issues and applications. Behavior Modification, 22, 221-242.

doi: $10.1177 / 01454455980223001$

Sencibaugh, J. M. (2007). Meta-analysis of reading comprehension interventions for students with learning disabilities: Strategies and implications. Reading Improvement, 44, 6-22. 
Sideridis, G. D. (2005). Performance approach-avoidance motivation and planned behavior theory: Model stability with Greek students with and without learning disabilities. Reading \& Writing Quarterly, 21, 331-359.

Simon-Cereijido, G., \& Gutiérrez-Clellen, V. F. (2014). Bilingual education for all: Latino dual language learners with language disabilities. International Journal of Bilingual Education and Bilingualism, 17, 235-254. doi: $10.1080 / 13670050.2013 .866630$

Slavin, R. E., \& Cheung, A. (2005). A synthesis of research on language of reading instruction for English language learners. Review of Educational Research, 75, 247-284. Retrieved from http://dx.doi.org/10/3102/00346543075002247

Snow, C. S., Burns, S. M., \& Griffin, P. (1998). Preventing reading difficulties in young children. Washington, DC: National Academy Press.

Snow, C. E., Lawrence, J. F., \& White, C. (2009). Generating knowledge of academic language among urban middle school students. Journal of Research on Educational Effectiveness, 2, 325-344.

Snyder, T. D., \& Dillow, S. A. (2012). Digest of education statistics 2011 (NCES-2012001). Washington DC: National Center for Education Statistics, Institute of Education Sciences, U.S. Department of Education.

Solari, E. J., \& Gerber, M. M. (2008). Early comprehension instruction for Spanishspeaking English language learners: Teaching text-level reading skills while maintaining effects on word-level skills. Learning Disabilities Research and Practice, 23, 155-168.

Stanovich, K. E. (1986). Matthew effects in reading: Some consequences of individual differences in the acquisition of literacy. Reading Research Quarterly, 21(4), 360407.

Swanson, H. (1999). Reading research for students with LD: A meta-analysis of intervention outcomes. Journal of Learning Disabilities, 32, 504-532.

Swanson, H. L., Hoskyn, M., \& Lee, C. (1999). Interventions for students with learning disabilities: A meta-analysis of treatment outcomes. New York, NY: Guilford.

Swanson, H., \& Malone, S. (1992). Social skills and learning disabilities: A metaanalysis of the literature. School Psychology Review, 21, 427-443. 
Swanson, H., Orosco, M., \& Lussier, C. (2012). Cognition and literacy in English language learners at risk for reading disabilities. Journal of Educational Psychology, 104, 202-230.

Talbott, E., Lloyd, J., \& Tankersley, M. (1994). Effects of reading comprehension interventions for students with learning disabilities. Learning Disability Quarterly, 17, 223-232.

Tam, K. Y., Heward, W. L., \& Heng, M. A. (2006). A reading instruction intervention program for English language learners who are struggling readers. The Journal of Special Education, 40, 79-93.

Thorius, K. K., \& Sullivan, A. L. (2013). Interrogating instruction and intervention in RTI research for students identified as English language learners. Reading and Writing Quarterly: Overcoming Learning Difficulties, 29(1), 64-88. doi:10.10/80/1057/3569.2013.741953

Trainor, A. A., \& Bal, A. (2014). Development and preliminary analysis of a rubric for culturally responsive research. The Journal of Special Education, 47, 203-216. doi: $10.1177 / 0022466912436397$

Trueba, H. T. (1988). Culturally based explanations of minority students' academic achievement. Anthropology and Education Quarterly, 19, 270-287. doi: 10.1525/aeq.1988.19.3.05x1565e

Vaughn, S., Linan-Thompson, S., Mathes, P. G., Cirino, P. T., Carlson, C. D., PollardDurodola, S. D., Cardenas-Hagan, E., \& Francis, D. J. (2006). Effectiveness of a Spanish intervention for first-grade English language learners at risk for reading difficulties. Journal of Learning Disabilities, 39, 56-73.

Vaughn, S., Mathes, P., \& Linan-Thompson, S. (2006). Effectiveness of an English intervention for first-grade English language learners at risk for reading problems. Elementary School Journal, 107, 153-180.

Wanzek, J., \& Roberts, G. (2012). Reading interventions with varying instructional emphases for fourth graders with reading difficulties. Learning Disability Quarterly, 35, 90-101.

Wanzek, J., Vaughn, S., Scammacca, N. K., Metz, K., Murray, C. S., Roberts, G., \& Danielson, L. (2013). Extensive reading interventions for students with reading difficulties after Grade 3. Review of Educational Research, 83(2), 163-195. 
WIDA Consortium. (2010). Assessing Comprehension and Communication in English State to State for English Language Learners: ACCESS for ELLs ${ }^{\circledR}$ : ACCESS interpretive guide for score reports. Madison, WI: WIDA Consortium.

Wong Fillmore, L. (2000). Loss of family languages: Should educators be concerned? Theory Into Practice, 39, 203-210. http://dx.doi.org/10.1207/s15430421tip3904_3

Woodcock, R. W., McGrew, K. S., \& Mather, N. (2001). Woodcock Johnson III Tests of Achievement. Itasca, IL: Riverside.

Yang, Y. (2006). Reading strategies or comprehension monitoring strategies? Reading Psychology, 27, 313-343. 


\section{APPENDIX A}

\section{RUBRIC FOR CULTURALLY RESPONSIVE RESEARCH}

TRAINOR AND BAL (2014)

\begin{tabular}{|c|c|c|c|}
\hline Component & 0 - does not meet & 1-partially meets & 2- meets \\
\hline $\begin{array}{l}\text { 1. Foundational } \\
\text { constructs of the } \\
\text { study }\end{array}$ & $\begin{array}{l}\text { The construct under } \\
\text { examination is implied } \\
\text { but not explicitly } \\
\text { discussed. }\end{array}$ & $\begin{array}{l}\text { The construct under } \\
\text { examination is explicit } \\
\text { but taken as universal } \\
\text { based on a norm- } \\
\text { referenced sample with } \\
\text { dominant } \\
\text { cultural/linguistic } \\
\text { background. Evidence of } \\
\text { alternative } \\
\text { conceptualizations is not } \\
\text { presented. }\end{array}$ & $\begin{array}{l}\text { The construct under } \\
\text { examination is addressed } \\
\text { comprehensively and } \\
\text { adequately; multiple } \\
\text { perspectives and/or } \\
\text { competing ideas are } \\
\text { presented with a } \\
\text { presentation of evidence } \\
\text { of alternative } \\
\text { conceptualizations. }\end{array}$ \\
\hline $\begin{array}{l}\text { 2. Relevancy of the } \\
\text { research problem }\end{array}$ & $\begin{array}{l}\text { The relevancy of the } \\
\text { research problem(s) to } \\
\text { participants' interests } \\
\text { and needs and context } \\
\text { is not discussed. }\end{array}$ & $\begin{array}{l}\text { The relevancy of the } \\
\text { research problem is } \\
\text { discussed, as it relates to } \\
\text { the field and/or the } \\
\text { researcher's interest or } \\
\text { line of inquiry. }\end{array}$ & $\begin{array}{l}\text { The relevancy of the } \\
\text { research problem } \\
\text { addresses both the } \\
\text { researcher's line of } \\
\text { inquiry and the } \\
\text { participants' and local } \\
\text { communities' interests } \\
\text { and needs. }\end{array}$ \\
\hline $\begin{array}{l}\text { 3. Critical and } \\
\text { comprehensive } \\
\text { review of relevant } \\
\text { literature. }\end{array}$ & $\begin{array}{l}\text { The review of extant } \\
\text { literature is a narrow } \\
\text { rationale for the study } \\
\text { that does not address } \\
\text { what is known about } \\
\text { the problem. }\end{array}$ & $\begin{array}{l}\text { The review of extant } \\
\text { literature includes } \\
\text { scholarship as it relates to } \\
\text { the research problem } \\
\text { relevant to the unit of } \\
\text { analysis. }\end{array}$ & $\begin{array}{l}\text { The review of extant } \\
\text { literature is critical and } \\
\text { creates a dialogue with } \\
\text { studies using alternative } \\
\text { methodologies and } \\
\text { perspectives on the } \\
\text { research problem relevant } \\
\text { to the unit of analysis. }\end{array}$ \\
\hline
\end{tabular}

(Table Continues) 


\begin{tabular}{|c|c|c|c|}
\hline Component & 0 -does not meet & 1-partially meets & 2- meets \\
\hline $\begin{array}{l}\text { 4. Description of } \\
\text { participants }\end{array}$ & $\begin{array}{l}\text { Description of } \\
\text { participants' } \\
\text { demographic } \\
\text { characteristics } \\
\text { includes two or fewer } \\
\text { characteristics (race, } \\
\text { gender, income, } \\
\text { disability). }\end{array}$ & $\begin{array}{l}\text { Description of } \\
\text { participants includes more } \\
\text { than two characteristics; } \\
\text { however, the description } \\
\text { is limited to the } \\
\text { dimension of the } \\
\text { individual (i.e., excludes } \\
\text { dimensions of the } \\
\text { institution). }\end{array}$ & $\begin{array}{l}\text { Description of } \\
\text { participants includes } \\
\text { both individual } \\
\text { characteristics and the } \\
\text { institutional dimensions } \\
\text { (e.g., status, } \\
\text { institutionalized social } \\
\text { practices), for both the } \\
\text { control groups and the } \\
\text { intervention groups. }\end{array}$ \\
\hline $\begin{array}{l}\text { 5. Description of } \\
\text { researchers and } \\
\text { interventionists }\end{array}$ & $\begin{array}{l}\text { Description of } \\
\text { researchers and } \\
\text { interventionists } \\
\text { includes two or fewer } \\
\text { individual } \\
\text { characteristics (e.g., } \\
\text { race, experience, } \\
\text { language). }\end{array}$ & $\begin{array}{l}\text { Description includes more } \\
\text { than two individual } \\
\text { characteristics (e.g., race, } \\
\text { gender, economic } \\
\text { background, disability); } \\
\text { however, the description } \\
\text { is limited to the } \\
\text { dimension of the } \\
\text { individual. }\end{array}$ & $\begin{array}{l}\text { Description includes } \\
\text { individual characteristics } \\
\text { and the contextualized } \\
\text { institutional dimension } \\
\text { and relational positions } \\
\text { among the participants } \\
\text { and interventionists (e.g., } \\
\text { power, status, and } \\
\text { insider/outsider } \\
\text { positions). }\end{array}$ \\
\hline $\begin{array}{l}\text { 6. Description of } \\
\text { sampling procedures }\end{array}$ & $\begin{array}{l}\text { Recruitment and } \\
\text { sampling methods are } \\
\text { not discussed. }\end{array}$ & $\begin{array}{l}\text { Recruitment and sampling } \\
\text { methods are discussed, } \\
\text { but lack detail about the } \\
\text { rationale for the } \\
\text { exclusionary criteria (e.g., } \\
\text { English learners) and the } \\
\text { congruence of } \\
\text { participants' experiences } \\
\text { and/or preferences (e.g., } \\
\text { language preference). }\end{array}$ & $\begin{array}{l}\text { Recruitment and } \\
\text { sampling methods } \\
\text { include differentiation } \\
\text { based on participants' } \\
\text { experiences and } \\
\text { preferences, maximizing } \\
\text { the potential to include } \\
\text { diverse populations. }\end{array}$ \\
\hline
\end{tabular}

(Table Continues) 


\begin{tabular}{|c|c|c|c|}
\hline Component & 0 - does not meet & 1-partially meets & 2- meets \\
\hline $\begin{array}{l}\text { 7. Description of data } \\
\text { collection strategies }\end{array}$ & $\begin{array}{l}\text { A rationale for data } \\
\text { collection strategies is } \\
\text { not discussed. }\end{array}$ & $\begin{array}{l}\text { A rationale for the data } \\
\text { collection strategies is } \\
\text { provided; however, it is } \\
\text { limited to a technical } \\
\text { discussion of the } \\
\text { methodology. }\end{array}$ & $\begin{array}{l}\text { A rationale for the data } \\
\text { collection strategies } \\
\text { includes consideration of } \\
\text { participants' cultural and } \\
\text { linguistic preferences, } \\
\text { needs, and strengths. } \\
\text { Multiple data collection } \\
\text { methods are used to } \\
\text { maximize accessibility } \\
\text { (e.g., using instruments in } \\
\text { multiple languages, using } \\
\text { participant-selected } \\
\text { locales). Description } \\
\text { includes discussion of } \\
\text { interactions between the } \\
\text { researchers and } \\
\text { participants. }\end{array}$ \\
\hline $\begin{array}{l}\text { 8. Ecology of the } \\
\text { intervention }\end{array}$ & $\begin{array}{l}\text { The intervention } \\
\text { includes a contrived } \\
\text { context, task, and } \\
\text { control for variables to } \\
\text { the extent that its } \\
\text { application in real life } \\
\text { is unlikely. }\end{array}$ & $\begin{array}{l}\text { The intervention includes } \\
\text { a context, task, and } \\
\text { variables that generally } \\
\text { represent participants' } \\
\text { real life experiences yet } \\
\text { the intervention aligns } \\
\text { more closely with } \\
\text { research design. }\end{array}$ & $\begin{array}{l}\text { The intervention is aligned } \\
\text { with participants' } \\
\text { experiences and/or } \\
\text { preferences. The integrity } \\
\text { of the participants' } \\
\text { experiences and contexts is } \\
\text { balanced with the } \\
\text { researchers' design. }\end{array}$ \\
\hline $\begin{array}{l}\text { 9. Assessment of } \\
\text { intervention efficacy }\end{array}$ & $\begin{array}{l}\text { The validity, } \\
\text { reliability, and } \\
\text { language of the } \\
\text { measurement tool(s) } \\
\text { are not discussed. }\end{array}$ & $\begin{array}{l}\text { The validity, reliability, } \\
\text { and language of the } \\
\text { measurement tool(s) are } \\
\text { discussed, but the } \\
\text { measurements are } \\
\text { standardized and norm- } \\
\text { referenced for a } \\
\text { population other than } \\
\text { sample. }\end{array}$ & $\begin{array}{l}\text { The validity, reliability, } \\
\text { and language of the tools } \\
\text { are inclusive of the } \\
\text { population representative } \\
\text { of participants OR the } \\
\text { limitation/lack of } \\
\text { availability of such tools } \\
\text { for the sample is discussed. }\end{array}$ \\
\hline
\end{tabular}

(Table Continues) 


\begin{tabular}{|c|c|c|c|}
\hline Component & 0 - does not meet & 1- partially meets & 2- meets \\
\hline $\begin{array}{l}\text { 10. Presentation of } \\
\text { findings }\end{array}$ & $\begin{array}{l}\text { The results are not } \\
\text { disaggregated } \\
\text { according to the } \\
\text { participant and setting } \\
\text { characteristics. }\end{array}$ & $\begin{array}{l}\text { The results are } \\
\text { disaggregated according } \\
\text { to participant } \\
\text { characteristics between } \\
\text { and within the } \\
\text { intervention and control } \\
\text { groups, but are limited to } \\
\text { disability, race, income, } \\
\text { or language. }\end{array}$ & $\begin{array}{l}\text { The results are } \\
\text { disaggregated to } \\
\text { participant characteristics } \\
\text { between and within the } \\
\text { intervention and control } \\
\text { groups and include } \\
\text { intersections of } \\
\text { participant characteristics. }\end{array}$ \\
\hline $\begin{array}{l}\text { 11. Analysis and } \\
\text { interpretation }\end{array}$ & $\begin{array}{l}\text { Culture-blind } \\
\text { approach: Participants' } \\
\text { cultural, linguistic, and } \\
\text { economic backgrounds } \\
\text { and contextual factors } \\
\text { are not included in } \\
\text { data analysis and } \\
\text { interpretation. }\end{array}$ & $\begin{array}{l}\text { Cultural deterministic } \\
\text { approach: Participants' } \\
\text { backgrounds and } \\
\text { contextual factors are } \\
\text { analyzed as categorical } \\
\text { and static variables. } \\
\text { Differences among } \\
\text { participants are } \\
\text { interpreted based on the } \\
\text { dis/advantages associated } \\
\text { with living conditions, } \\
\text { demographic } \\
\text { characteristics, or } \\
\text { participants' lack of } \\
\text { competencies in } \\
\text { mainstream skills and } \\
\text { knowledge. }\end{array}$ & $\begin{array}{l}\text { Cultural instrumentalist } \\
\text { approach: Participants' } \\
\text { backgrounds, contextual, } \\
\text { and cultural factors are } \\
\text { analyzed as dynamic, } \\
\text { complex, and dialogical. } \\
\text { Differences within the } \\
\text { participants are } \\
\text { interpreted as situated in } \\
\text { affordances and } \\
\text { constraints of the } \\
\text { physical, sociocultural, } \\
\text { and historical relations of } \\
\text { the context. Factors under } \\
\text { consideration include } \\
\text { organizational structures, } \\
\text { power distribution, and } \\
\text { participants' identities. }\end{array}$ \\
\hline $\begin{array}{l}\text { 12. Discussion of } \\
\text { dissemination }\end{array}$ & $\begin{array}{l}\text { Dissemination } \\
\text { strategies are limited } \\
\text { to the presentation of } \\
\text { data in the article. }\end{array}$ & $\begin{array}{l}\text { Dissemination strategies } \\
\text { extending beyond the } \\
\text { article are discussed (e.g., } \\
\text { the data were shared with } \\
\text { teachers and families). }\end{array}$ & $\begin{array}{l}\text { Dissemination strategies } \\
\text { are strategically selected } \\
\text { to maximize sharing of } \\
\text { knowledge with clear, } \\
\text { obvious benefits to } \\
\text { participants and } \\
\text { communities. }\end{array}$ \\
\hline
\end{tabular}




\section{APPENDX B \\ COMPREHENSION THINKING STRATEGY RUBRIC}

During each session, use the rubric to reflect the student's performance with the targeted strategy during each lesson.

Monitoring comprehension rubric (Keene, 2006)

Pose questions to student after reading:

- What problems did you have while reading?

- What did you do to solve problems you had while reading?

- How do you know when you understand text?

After listening to responses and observing reading behavior, choose the performance level that best reflects the student's performance.

Level Criteria

1 Student has little or no conscious awareness of reading process.

2

Student identifies difficulties- problems at word level; little or no sense of the need to solve the problem; does not articulate strengths; identifies need to concentrate; talks about word-level solutions (sounding it out) for textlevel comprehension problems. Student identifies problems at word, sentence, or schema level: can articulate and use a strategy to solve problems, usually at the word or sentence level.

4 Student articulates and uses more than one strategy for solving problems; focuses on problems at the whole-text level.

5 Student identifies problems at all levels; uses a variety of word level and comprehension strategies flexibly and appropriately given the context and the problem. 


\section{APPENDIX C \\ COMPREHENSION QUESTION FRAMES}

\section{Literal question frames}

1. What does the word mean in this passage? (when definition is provided in context or in a glossary) or What word was used to describe (attribute of character or setting) in this passage?

2. What ? (e.g., What helps a giraffe run fast? What did the class do on the field trip?)

3. Who or when ? (e.g., Who is the main character? When does a stingray use its tail like a whip?)

4. Where ? (e.g., Where do Francisco and his grandfather work? Where do sea otters find their food?)

5. Which of these happened first? Or ask for objective information (e.g., Glaciers form when ).

\section{Inferential question frames}

1. What could be another title for this passage?

2. What is the problem? What is this passage trying to explain?

3. What lesson did the main character learn? Which is most likely true about $?$

4. From this passage, what can you infer about ? (e.g., Komodo dragons)

5. What can you conclude about ? (e.g., animals that sting) 


\section{APPENDIX D \\ MOTIVATION TO READ PROFILE: READING SURVEY}

Name:

Date:

Sample 1: I am in

Sample 2: I am a

second grade

third grade

fourth grade

fifth grade

__sixth grade

Item 1: My friends think I am

boy

girl

a very good reader

a good reader

an $\mathrm{OK}$ reader

not a good reader

Item 2: Reading a book is something I like to do.

never

not very often

sometimes

Item 3: I read

often

__ not as well as my friends.

about the same as a few of my friends.

about the same as most of my friends.

_a lot better than my friends.

Item 4: My friends think reading is

really fun

fun

OK to do

no fun at all 
Item 5: When I come to a word I do not know, I can almost always figure it out sometimes figure it out _almost never figure it ou _never figure it out

Item 6: I tell my friends about good books I read.

I never do this.

I almost never do this.

I do this some of the time.

I do this a lot.

Item 7: When I am reading by myself, I understand almost everything I read some of what I read almost none of what I read none of what I read

Item 8: People who read a lot are

Item 9: I am _

very interesting

interesting

not very interesting

boring

Item 10: I think libraries are

_not a good reader

an OK reader

a good reader

a very good reader

a great place to spend time.

an interesting place to spend time

_ an OK place to spend time

__a boring place to spend time

Item 11: I worry about what other kids think about my reading

every day

_almost every day

once in a while

never 
Item 12: Knowing how to read well is

_not very important
_sort of important
_ important
_ very important

Item 13: When my teacher asks me a question about what I have read, I can never think of an answer have trouble thinking of an answer sometimes think of an answer _always think of an answer

Item 14: I think reading is

a boring way to spend time an OK way to spend time an interesting way to spend time a great way to spend time

Item 15: Reading is

_very easy for me

kind of easy for me

_ kind of hard for me

_ very hard for me

Item 16: When I grow up I will spend

__ none of my time reading

_ very little of my time reading

__some of my time reading

_ a lot of my time reading

Item 17: When I am in a group talking about stories, I __almost never talk about my ideas __sometimes talk about my ideas __almost always talk about my ideas _always talk about my ideas

Item 18: I would like my teacher to read books out loud to the class

_every day

- almost every day

once in a while

- never 
Item 19: When I read out loud I am a

not good reader

OK reader

good reader

very good reader

Item 20: When someone gives me a book for a present, I feel

_very happy
_sort of happy
__sort of unhappy
__unhappy

$<$ end of page $>$ 


\section{Scoring Guide}

Recoding scale:

$$
\begin{aligned}
& 1=4 \\
& 2=3 \\
& 3=2 \\
& 4=1
\end{aligned}
$$

Self-concept as a reader recode 1 .

3.

recode 5 .

recode 7 .

9.

recode 11 .

13.

recode 15 .

17.

19.

140
Value of reading

2.

recode 4 .

6.

recode 8 .

recode 10 .

12.

14.

16.

recode 18. recode 20 .

Value score 140

Total score:

180

Comments:

Gambrell, L.B., Palmer, B.M., Codling, R.M., Mazzoni, S.A. (1996). Assessing reading motivation: The Reading Teacher 49(7), 518-533. 


\section{APPENDIX E}

\section{MOTIVATION TO READ PROFILE: READING INTERVIEW}

1. Tell me about a book you read at home or at school this week.

2. Do you know of any books that you would like to read?

3. How did you know or find out about this book?

4. What are some things that good readers do?

5. What are some things that people can do to become better at reading?

6. What do you need to learn to be a better reader?

Adapted from the Motivation to Read Profile: Conversational Profile by Gambrell, Palmer, Codling, and Mazzoni (1996) 


\section{APPENDIX F}

\section{PRE- AND POSTTEST DATA RECORDING FORM}

Participant (no actual names): Grade level:

Composite ACCESS Score from 2014: SED Eligibility:

IEP Goal Areas: Initial Date:

Woodcock Johnson Pretest Data

date SS

Woodcock Johnson Posttest Data date SS

Fountas and Pinnell Benchmark Assessment Date Assessed:

\begin{tabular}{|l|l|l|}
\hline Independent reading level: & Instructional level: & Frustration level: \\
\hline
\end{tabular}

Fountas and Pinnell Benchmark Assessment Date Assessed:

\begin{tabular}{|l|l|l|}
\hline Independent reading level: & Instructional level: & Frustration level: \\
\hline
\end{tabular}

Results from Pre- and Postintervention Interview:

Date administered:

Notes:

Date administered:

Notes: 
APPENDIX G

SOCIAL VALIDATION SURVEY

\begin{tabular}{l}
\hline $\begin{array}{l}\text { Put a check in the box that shows } \\
\text { how much you agree or disagree } \\
\text { with each statement. }\end{array}$
\end{tabular}

I liked using iThoughts HD to

make idea maps during this

reading group.

I liked using kidblog to share my

thoughts about texts I read during

this reading group.

I liked using Educreations during

this reading group to narrate my

use of reading strategies.

I enjoyed the books that I read

during this reading group.

The characters in the fiction texts

that I read were very much like me

and my family.

I often read, view, or listen to texts

that are very similar to the ones I

read during this reading group.

(Table Continues) 
Put a check in the box that shows

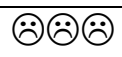

:००

2)

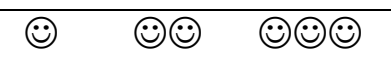

how much you agree with each

statement.

Using the TRACK strategy helped

me understand what I was reading.

The self-monitoring procedure

helped me remember to use

strategies while reading.

Coding the text helped me keep

track of my thinking. 
APPENDIX H

FORMATIVE ASSESSMENT DATA RECORDING FORM

\begin{tabular}{|c|c|c|}
\hline $\begin{array}{l}\text { Participant: } \\
\text { Text: }\end{array}$ & $\begin{array}{l}\text { Date: } \\
\text { Group Size: }\end{array}$ & $\begin{array}{l}\text { Lesson Beginning time: } \\
\text { Lesson Ending time: }\end{array}$ \\
\hline $\begin{array}{l}\text { Baseline } \\
\text { Intervention } \\
\text { Generalization } \\
\text { Maintenance } \\
\text { This lesson used } \\
\text { modeling } \\
\text { guided practice } \\
\text { independent practice } \\
\\
\text { Target Skill Rubric Score: } \\
\text { Monitoring } \\
\text { comprehension } \\
\text { Questioning } \\
\text { Using Schema } \\
\text { Self-monitoring procedure: } \\
\text { not introduced } \\
\text { modeled } \\
\text { guided practice } \\
\text { independent practice }\end{array}$ & $\begin{array}{l}\begin{array}{l}\text { Responses to low-level } \\
\text { (literal) questions: }\end{array} \\
\% \text { accuracy } \\
\begin{array}{l}\text { Responses to high-level } \\
\text { (inference) questions: } \\
\% \text { accuracy }\end{array} \\
\text { Total comprehension } \\
\% \text { accuracy } \\
\text { Word reading } \\
\text { For the next lesson, the } \\
\text { instructional-level text } \\
\text { should: } \\
\text { stay the same } \\
\text { increase } \\
\text { decrease }\end{array}$ & $\begin{array}{l}\text { Notes on affect and use } \\
\text { of target skill: }\end{array}$ \\
\hline
\end{tabular}




\section{APPENDIX I}

\section{PROCEDURAL RELIABILITY CHECKLIST FOR BASELINE}

\section{AND MAINTENANCE CONDITIONS}

\begin{tabular}{|c|c|c|c|}
\hline \multicolumn{2}{|c|}{ Participant:_ $\quad$ Observer: } & & \\
\hline \multicolumn{4}{|c|}{ Date:_ Number of components successfully completed:__/7 } \\
\hline \multicolumn{4}{|l|}{ Text:___ } \\
\hline \multirow{2}{*}{\multicolumn{4}{|c|}{$\begin{array}{ll}\text { Number of literal questions answered correctly: } & / 5 \\
\text { Number of inferential questions answered correctly: } & / 5\end{array}$}} \\
\hline & & & \\
\hline \multicolumn{4}{|c|}{ Rubric Score: ${ }^{1}{ }^{2}-3{ }^{3}-4$} \\
\hline Sequence & Teaching Actions & YES & NO \\
\hline $\begin{array}{l}\text { Before } \\
\text { Reading }\end{array}$ & $\begin{array}{l}\text { 1. The instructor will ask participants to scan the text and generate } \\
\text { predictions. }\end{array}$ & & \\
\hline During & $\begin{array}{l}\text { 2. The instructor will direct participants to use choral, echo, or } \\
\text { silent reading to read to a designated stopping point. }\end{array}$ & & \\
\hline \multirow[t]{2}{*}{ Reading } & $\begin{array}{l}\text { 3. At the designated stopping point, the instructor will ask } \\
\text { participants to make predictions about what could happen next. }\end{array}$ & & \\
\hline & $\begin{array}{l}\text { 4. The instructor will have participants continue to read silently } \\
\text { from instructional-level text. }\end{array}$ & & \\
\hline \multirow{4}{*}{$\begin{array}{l}\text { After } \\
\text { Reading }\end{array}$} & 5. The instructor will pose scripted literal and inferential & & \\
\hline & $\begin{array}{l}\text { comprehension questions. Paraphrase responses. Record }(+) \text { for } \\
\text { on-target responses and (-) for off-target responses on data sheets, } \\
\text { along with date, title of text, and genre of text. }\end{array}$ & & \\
\hline & $\begin{array}{l}\text { 6. The instructor will ask participants to talk about the text as well } \\
\text { as to explain how he or she monitored comprehension; listen to } \\
\text { responses. Assign a rubric score. Record rubric score and targeted } \\
\text { strategy on data sheet. }\end{array}$ & & \\
\hline & $\begin{array}{l}\text { 7. The instructor will have participant verbally pose questions, } \\
\text { make comments, and offer insights about what he or she read by } \\
\text { posting to a weblog or by writing in a reader's response notebook. }\end{array}$ & & \\
\hline
\end{tabular}




\section{APPENDIX J}

\section{PROCEDURAL RELIABILITY CHECKLISTS FOR INTERVENTION LESSONS}

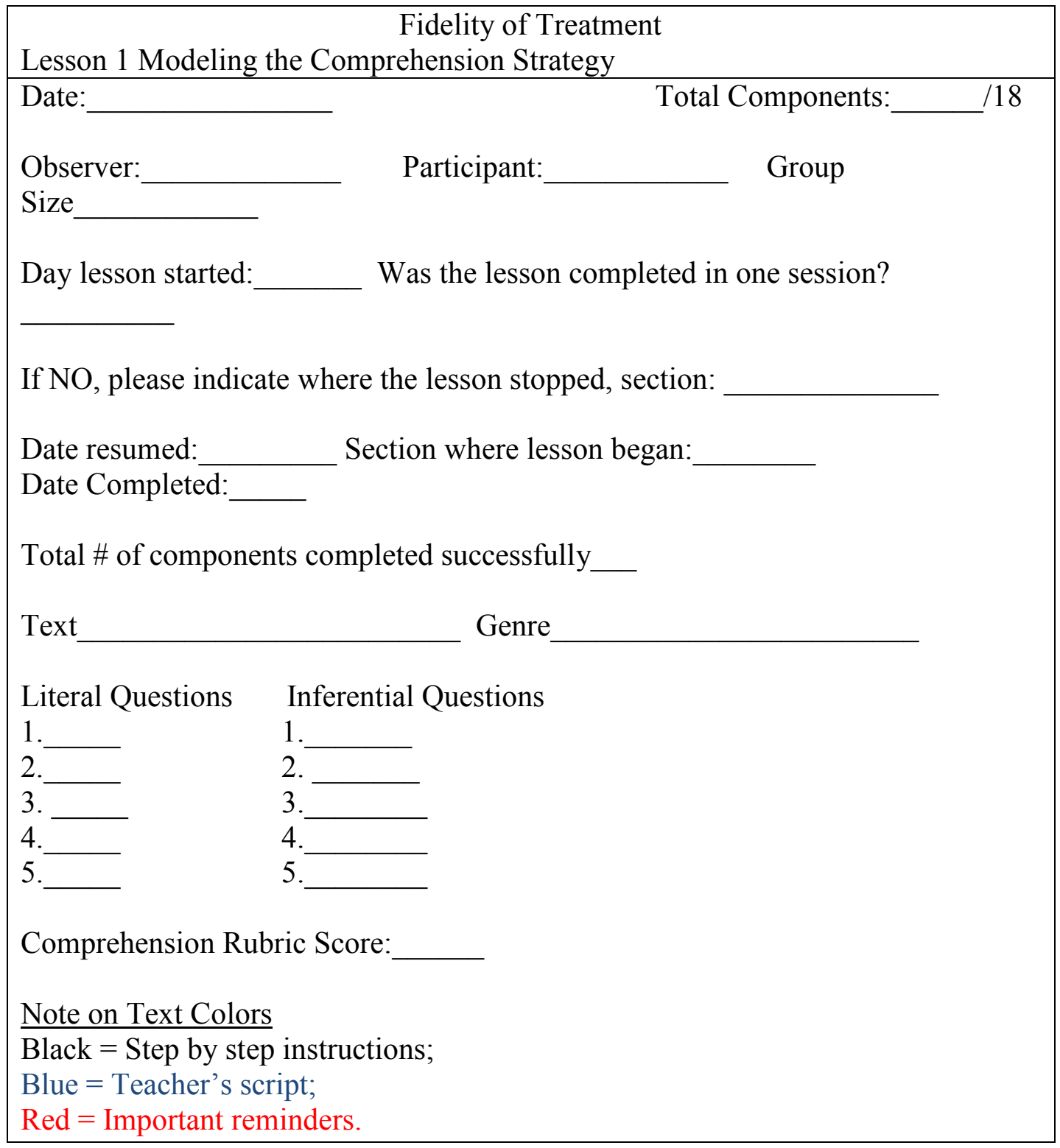


Fidelity of Treatment

\section{Lesson 1 Modeling the Comprehension Strategy}

1. The lesson begins by setting goals.

2. Instructor presents the agenda.

Agenda:

- Listen to the story A Day's Work

- Learn how to use TRACK to code the text

- Read text from book boxes

- Share my thinking about the text

- Reflect on my goal

- Create a kidblog post to share my responses to text

3. The instructor presents the mentor text. Today, I will read aloud from a story called A Day's Work by Eve Bunting. This story shows us how honesty and integrity are smart choices in any situation.

4. The instructor presents the mentor text and asks questions to activate prior knowledge on the text topic; participant uses mind-mapping applications to create idea webs. Have you ever heard the expression honesty is the best policy? There are many ways to solve a problem. Honesty is one of the best solutions. What do you think it means if you tell someone about a problem, such as forgetting your homework, and they say honesty is the best policy? How could honesty solve the problem of not having your homework? What are some other examples of situations where honesty is the best policy?

5. The instructor introduces the comprehension strategies for monitoring for meaning. Today I will show you a strategy called text coding. Text coding is a strategy that readers can use to reflect on and react to what they read. When I code the text, I leave tracks of my thinking. This means that you will be able to see what I was thinking while I was reading a text.

6. The instructor states the purpose of the comprehension strategies. Nothing is more important during reading than the reader's thinking. I will read the book $A$ Day's Work with you today because it makes me think about so many things. When readers pay attention and think about the words and ideas in text, they have an inner conversation with the text. It is a quiet conversation that happens only in the reader's head. Today, when I code the text, I will leave tracks so that you can follow my thinking. 
7. The instructor explains sentence-starters and explains the TRACK mnemonic. I will use the word TRACK to remind me of the steps I need to follow as I code the text. Displays the TRACK mnemonic by uncovering one letter at a time.

Reading Strategy: Code the text to keep TRACK of your thinking.

Think about what I am reading.

React to the text.

Ask questions.

Connect.

Keep track of your thinking.

The first letter in the word TRACK is "T." The letter "T" reminds me to think about what I am reading. Today, I will show you how I pay attention to my thoughts as I read. For example, I might read something that confuses me and think, "Huh, I don't get this part." I might read something that tells me new information and think, "Wow, I never knew that before." The letter " $\mathrm{T}$ " reminds me to think while I read so I can have an inner conversation with the text. Ask for preference between "think about what I am reading" and "think while I read."

The second letter in the word TRACK is "R." The letter "R" reminds me to react to the text. It is not enough to just read and think about the text. I also have to react and explore my thinking. This means that when I notice my thinking, I also do something about it. For example, if I notice that I am confused by a word that is hard to read, I will do something about it. I might read ahead and use context clues to try to figure out what the word means or I might circle the word and decide to look it up in the dictionary. Today, when I think aloud about what I am reading you might know that I am going to react to my thinking if you hear me say, "I am confused about ." [Hold up sentence-starter written on a sentence strip.] The letter "R" reminds me to do more than just read and think about the text-it tells me to react. Ask for preference between "react to the text" and respond to the text." 
The third letter in the word TRACK is "A." The letter "A" reminds me to ask questions. Asking questions and wondering about text is part of what good readers do. Some questions that readers ask can be answered easily in few words. Others are big questions that have long, involved answers. And other questions can't be answered at all. Today, when I show you how I ask questions while I read, you might hear me say, "I wonder why the author chose to ." [Hold up sentence-starter written on a sentence strip.] The letter "A" reminds me that it is important to ask questions while I read. Ask for preference between "ask questions" and "ask questions to the author".

The fourth letter in the word TRACK is "C." The letter " $\mathrm{C}$ " reminds me to connect to what I am reading. Good readers make connections between the books they read and their own lives. Today, I will show you how I make connections while reading. You will now that I am going to connect to what I am reading when you hear me say, "This reminds me of

." [Hold up sentence-starter written on a sentence strip.] The letter "C" reminds me to connect my own knowledge and experiences to what I am reading. Ask for preference between "connect" and "connect to what I am reading."

The final letter in the word TRACK is "K." The letter "K" reminds me to keep track of my thinking. To keep track of my thinking, I will code the text. I will use symbols to show what I am thinking. I will write a symbol on a sticky note and place the sticky note on the page of the text that made me react. Ask for preference between "Keep track of my thinking" and "keep track of what I think while I read." Some of the symbols I might use are:

i. "??" to show that I am confused or that I am wondering something

ii. " "!?" to show that I read something that I think is interesting

iii. “*” to show that I made a connection to something I already know

8. The instructor reads aloud from mentor text until reaching a predetermined stopping point. I am going to read aloud from A Day's Work. While I read, I will think aloud and I will code the text to leave tracks of my thinking. Your job is to listen to the story, listen to my thinking, and notice how I code the text. Begins reading aloud the first page of the story. Stops on page two mid-way through the page before the paragraph that begins with "Francisco swallowed..."

9. The instructor thinks aloud by using the sentence-starters that accompany comprehension strategies. Holds up sentence-starter. "I am confused about why Francisco is standing with his grandfather in a parking lot on a Saturday morning." 
10. The instructor models using sticky notes to code the text and to leave tracks of thinking while verbally explaining how and why to code text. What I am reading does not make sense to me yet, I am going to code the text with "??" symbols to remind myself that I can use strategies to figure this out. I will read ahead and see if I can figure out why Francisco is waiting with his grandfather. Continues reading and stop at the bottom of page 6 after the sentence that ends "skinny as an old tree." Now that I have read ahead, I figured out that Francisco was there to help his grandfather find work for the day. I wonder why the author chose to describe the grandfather using the simile "as skinny as an old tree. Codes the text with ?? When a tree gets very old, its branches become weak. It makes me think that maybe the author wants me to think that Francisco's grandfather is delicate or fragile, easily broken.

11. The instructor continues reading aloud from mentor text, stopping to think aloud. Reads aloud; stops at the bottom of page 10. It seems like Francisco is very brave. The author described how Francisco pushed a tough guy out of the way. I think the tough guy was going to try to steal the job from Francisco and his grandfather. Holds up sentencestarter. This reminds me of how I can surprise myself by acting bravely when I really want to accomplish something. Codes text with a * and continues reading aloud and stops in the middle of page 20 after the sentence, "Francisco and his grandfather shook hands." I can tell that Francisco and his grandfather are proud of all the hard work they accomplished. When the author wrote that Francisco and his grandfather shook hands, it reminded me of what I see a team do after playing hard in a game. Codes the text with a * symbol.

12. The instructor, while reading, models "fix up" strategies at word level, text level, $\overline{\text { and }}$ schema level (e.g., decode difficult words, re-read, read ahead, ask questions, or make connections). Continues reading aloud from the text and stops near the end of page 22 after reading aloud the sentence, "You took out my young ice plants."

I am confused. I think I need to read that sentence again. "You took out my young- ice- plants." I am not sure what an ice plant is. Codes text with ?? symbols. It seems like Ben is not happy about the work that Francisco and his grandfather did. I am going to read on to see if I can tell why Ben is upset. Continues reading to the end of the page. Okay, Ben is definitely upset. The author wrote that Ben slammed his Lakers hat against the van. This reminds me of what I have seen people do when they are really angry. But, I am still not sure why Ben is so angry. Codes text with * and ??; continues reading aloud through the top of page 30, to the sentence that ends, "we take the pay tomorrow, when we finish." I am starting to think that maybe I was off-track when I thought that the author compared the grandfather to an old tree to make me think that he is fragile. Reading about how abuelo handled this problem made me change my mind. He showed strength and integrity by not taking the money and by offering to come back to fix the mistake. Maybe old trees are stronger than I thought. Codes the 
text with !! to show that I read something that made me think differently; continues reading to the end of the story.

13. The instructor has the participant select and read an instructional-level text. Each student in the group reads a different text. Provides students with sticky notes to code their thinking. Provides visual cues to show how to code the text. Rotates through the group and has students read portions of the text aloud. Notes the level of word-reading accuracy demonstrated by participant (number of words read correctly divided by the total number of words read aloud). Records the date, the title of the text, the genre of the text, and other relevant information about the lesson on the formative assessment data recording form.

For the next 10 minutes each of you will read from a book in your book box. As you read, use sticky notes to code the text. You can use the symbols ??. !! and * to leave tracks of your thinking. Begin reading silently. I will ask some of you to read aloud to me. Any questions? [Answers questions.] Begin."

14. The instructor poses scripted literal and inferential comprehension questions. Paraphrases participants' responses. Records $(+)$ for on-target responses and (-) for offtarget responses on data sheets, along with date, title of text, and genre of text.

15. The instructor asks participant to talk about the text as well as to explain how he or she used the targeted comprehension strategy. Uses scripted prompts, including:

- What problems did you have while reading?

- What did you do to solve problems you had while reading?

- How do you know when you understand text?

16. The instructor, after listening to responses and observing reading behavior, chooses the performance level that best reflects the student's performance (i.e., assigns a rubric score.) Records rubric score and targeted strategy on data sheet. 


\begin{tabular}{|l|l|}
\hline Level & Criteria \\
\hline 1 & Student has little or no conscious awareness of reading process. \\
\hline 2 & $\begin{array}{l}\text { Student identifies difficulties- problems at word level; little or no sense } \\
\text { of the need to solve the problem; does not articulate strengths; identifies } \\
\text { need to concentrate; talks about word-level solutions (sounding it out) } \\
\text { for text-level comprehension problems. }\end{array}$ \\
\hline 3 & $\begin{array}{l}\text { Student identifies problems at word, sentence, or schema level: can } \\
\text { articulate and use a strategy to solve problems, usually at the word or } \\
\text { sentence level. }\end{array}$ \\
\hline 4 & $\begin{array}{l}\text { Student articulates and uses more than one strategy for solving } \\
\text { problems; focuses on problems at the whole-text level. }\end{array}$ \\
\hline 5 & $\begin{array}{l}\text { Student identifies problems at all levels; uses a variety of word level and } \\
\text { comprehension strategies flexibly and appropriately given the context } \\
\text { and the problem. }\end{array}$ \\
\hline
\end{tabular}

17. The instructor asks the participant to reflect on his or her goal. Look at the goal you recorded at the beginning of the session. Think about the progress you made toward meeting your goal. Make a note to show whether you met your goal.

18. The instructor has participants verbally pose questions, make comments, and offer insights by posting to a weblog or by writing in a reader's response notebook. 
Fidelity of Treatment

Lesson 2 Modeling the Self-Monitoring Procedure

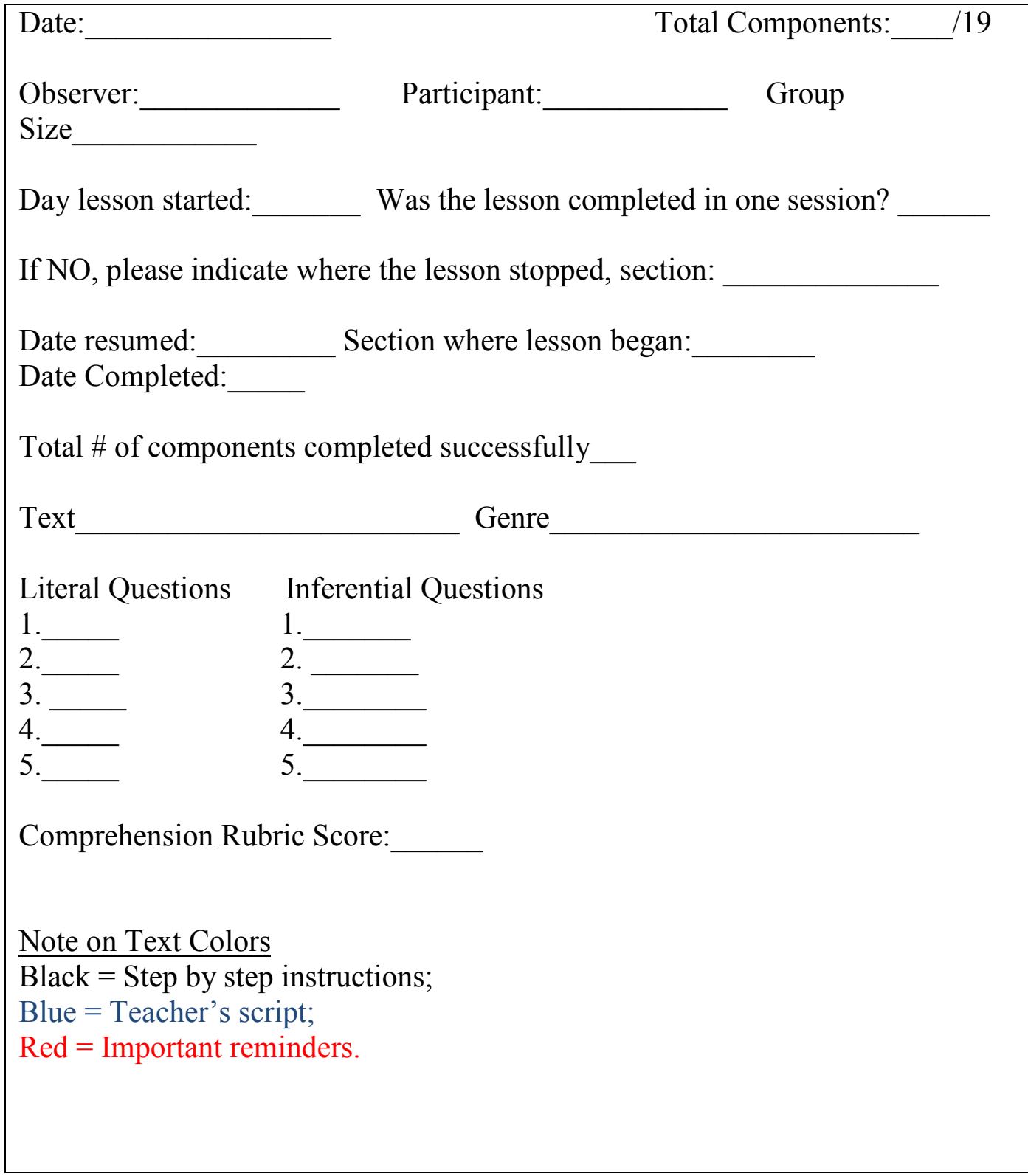


Fidelity of Treatment

\section{Lesson 2: Modeling the Self-Monitoring Procedure}

1. The instructor begins the lesson will by having students set goals.

2. The instructor presents the agenda.

Agenda:

- Listen to the story The Day of Ahmed's Secret

- Read text from book boxes

- Share my thinking about the text

- Reflect on my goal

- Create a kidblog post to share my responses to text

3. The instructor presents mentor text. Today, I will read aloud from a story called The Day of Ahmed's Secret by Ted Lewin. This story is about a boy who waits very patiently all day for a special moment.

4. The instructor asks questions to activate prior knowledge and has students use mind-mapping applications to create idea webs. Can you think of something that you have waited patiently for? Makes a web of ideas. Praises students for contributing ideas to the web.

5. The instructor revisits the comprehension strategies for monitoring for meaning. We will use the text coding strategy as we read. The word TRACK can help us remember to code the text and to monitor for meaning while we read.

6. The instructor states the purpose of the comprehension strategies. Remember that this strategy helps us read and comprehend complex literary and informational text (CCSS. ELA-Literacy.CCRA.R.10). When we code the text, we leave tracks of our thinking. We use this strategy to reflect on and react to text.

7. The instructor displays the TRACK mnemonic and sentence-starters related to the comprehension strategies. Last time we met, we used sentence-starters to talk about the strategy that we used while thinking aloud about the text. Holds up sentence-starters on sentence strips. For the strategy ask questions, we started sentences with ... Has students select the corresponding sentence strip. Provides praise for attempting/achieving the task. For the strategy connect we used the sentence-starter... Has students select the corresponding sentence strip. Provides praise for attempting/achieving the task. When we monitored comprehension and realized that what we read did not make sense, we used.... Have students select the corresponding sentence strip. Provide praise for attempting/achieving the task. What key word do we use to remind us how to TRACK our thinking? Listen to responses, and accept TRACK. Let's see if we can remember what each of the letters in the word TRACK stand for. Present reading comprehension 
strategy cue card. Cover up the card and reveal its contents letter by letter as students collaboratively recall the strategy. Provide praise for each attempt to recall the strategy.

Reading Strategy: Code the text to keep TRACK of your thinking.

Think about what I am reading.

React to the text.

Ask questions.

Connect.

Keep track of your thinking.

8. The instructor presents the self-monitoring checklist. Today you will learn four steps to help you use the TRACK strategy so you can monitor for meaning while you read. Let's look at the self-monitoring procedure card to learn what the four steps are.

$\square$ I read the paragraph.

$\square$ I used the prompt card to recall the strategy steps.

$\square$ I applied the strategy to monitor for meaning.

$\square$ I coded the text to leave tracks of my thinking.

9. The instructor states the purpose of the self-monitoring checklist. This card will help you use the TRACK strategy. You will use the card to make sure that you completed all of the steps involved in coding the text. Coding the text is a strategy that good readers use to comprehend what you read. I will show you how I use the card to monitor how I use the text coding strategy.

10. The instructor reads aloud from mentor text. Opens the book The Day of Ahmed's Secret and reads aloud the first paragraph (which happens to be only one sentence long).

11. The instructor models checking off this step on the checklist. Checks off the first step on the self-monitoring procedure checklist.

12. The instructor reads aloud step 2 on the self-monitoring procedure card. Refers to the TRACK strategy card and thinks aloud about the first paragraph.

$\mathrm{T}$ means think while reading and $\mathrm{R}$ means react to the text. I am curious about what his secret could be. He says the secret will be like a friend to him. A stands for ask questions. What does the author mean about the secret being like his friend? I think this means he is going to keep it very close to him and not give anyone any hints about what it could be. $\mathrm{C}$ stands for connect. This reminds me of how I feel when I know about something (like a surprise party) that I need to keep a secret. I am going 
to code the text "!!"” because I like the way the author compared having a secret to having a friend. I used the prompt card to recall the steps for TRACK, so I will check off step two. I used each letter to think about the text. So, I will check off step three on the checklist. I coded the text to leave tracks of my thinking, so I will check off step four. Now, I am going to keep reading the story until I reach a point where I want to ask questions or make connections.

Continues reading the story and modeling the self-monitoring procedure.

13. The instructor restates the purpose of the self-monitoring procedure. The four steps on the self-monitoring procedure card will help you use the TRACK strategy so you can monitor for meaning while you read.

14. The instructor has the participant select and read an instructional-level text. Each student in the group reads a different text. Provides students with sticky notes to code their thinking. Provides visual cues to show how to code the text. Rotates through the group and have students read portions of the text aloud. Notes the level of word-reading accuracy demonstrated by participants (number of words read correctly divided by the total number of words read aloud). Records the date, the title of the text, the genre of the text, and other relevant information about the lesson on the formative assessment data recording form.

For the next 10 minutes each of you will read from a book in your book box. As you read, use sticky notes to code the text. You can use the symbols ??. !! and * to leave tracks of your thinking. Begin reading silently. I will ask some of you to read aloud to me. Any questions? [Answers questions.] Begin.

15. The instructor poses scripted literal and inferential comprehension questions and paraphrases the participant's responses. Records (+) for on-target responses and (-) for off-target responses on data sheets, along with date, title of text, and genre of text.

16. The instructor asks participant to talk about the text as well as to explain how he or she used the targeted comprehension strategy. Uses scripted prompts, including:

- What problems did you have while reading?

- What did you do to solve problems you had while reading?

- How do you know when you understand text?

17. The instructor, after listening to responses and observing reading behavior, chooses the performance level that best reflects the student's performance (i.e., assigns a rubric score) and records a rubric score on data sheet.

18. The instructor asks participant to reflect on his or her goal. Look at the goal you recorded at the beginning of the session. Think about the progress you made toward meeting your goal. Make a note to show whether you met your goal. 
19. The instructor has participant verbally pose questions, make comments, and offer insights by posting to a weblog or by writing in a reader's response notebook. 


\section{Fidelity of Treatment}

Lesson 3 Guided Practice with the Comprehension Strategy using Mentor Text Date: Total Components: $/ 18$

Observer:

Size

Participant:

Group

Day lesson started:

Was the lesson completed in one session?

If NO, please indicate where the lesson stopped, section:

Date resumed: Section where lesson began:

Date Completed:

Total \# of components completed successfully

Text Genre

\section{Literal Questions}

1.

2.

3.

4.

5.

Inferential Questions

1.

2.

3.

4.

5 .

Comprehension Rubric Score:

\section{Note on Text Colors}

Black = Step by step instructions;

Blue = Teacher's script;

Red $=$ Important reminders. 


\section{Fidelity of Treatment}

\section{Lesson 3: Guided Practice with Comprehension Strategy using Mentor Text}

1. The instructor starts the lesson by having students set goals

2. The instructor presents the agenda.

Agenda:

- Listen to the story One Green Apple

- Use TRACK to code the text

- Read text from book boxes

- Share my thinking about the text

- Reflect on my goal

- Create a kidblog post to share my responses to text

3. The instructor presents mentor text. Today, I will read aloud from a story called One Green Apple by Eve Bunting. This story will remind us of what we learned in about in our Second Step Lesson on empathy. Does anyone remember what empathy means? Listens to ideas. Provides verbal praise for sharing responses.

4. The instructor asks questions to activate prior knowledge on the text topic; has participant use mind-mapping applications to create idea webs. How could we show empathy to a new student who joins our class but does not speak English yet? Make a web of ideas. Praises students for contributing ideas.

5. The instructor revisits the comprehension strategies for monitoring for meaning. Today we are going to practice the text coding strategy. This is a strategy that will help us read and comprehend complex literary and informational text. (CCSS. ELA-Literacy.CCRA.R.10).

6. The instructor restates the purpose of the comprehension strategies. When we code the text, we leave tracks of our thinking. This strategy helps us to reflect on and react to text. Using TRACK helps us monitor for meaning while we read.

7. The instructor revisits sentence-starters related to the comprehension strategy. Last time we met I used sentence-starters to talk about the strategy that I was using while thinking aloud about the text. Holds up sentence-starters on sentence strips. For the strategy ask questions, I started sentences with ... Has students select the corresponding sentence strip. Provides praise for attempting/achieving the task. For the strategy "connect" I used the sentence-starter... Has students select the corresponding sentence strip. Provides praise for attempting/achieving the task. When I was monitoring my comprehension and realized that what I read did not make sense, I used.... Has students select the corresponding sentence strip. Provides praise for attempting/achieving the task.

8. The instructor presents the TRACK mnemonic. Asks, "What key word do we use to remind us how to TRACK our thinking?" Listens to responses, and accepts TRACK. Let's see if we can 
remember what each of the letters in the word TRACK stand for. Presents reading comprehension strategy cue card. Covers up the card and reveals its contents letter by letter as students collaboratively recall the strategy. Provides praise for each attempt to recall the strategy.

9. The instructor reads aloud from a mentor text until reaching a predetermined stopping point. I am going to read aloud from One Green Apple. While I read, your job is to listen and think. Follow along in your copy of this story so that you can code the text. At some points, I will stop reading so that we can share our thinking. What is your task? Listens to responses. Let's begin. Starts reading and stops at page 6 . Thinks aloud saying, "I wonder what the author means when she writes that the students looked at the girl coldly. Why do you think some kids would look at her like that?" Listens to and accepts responses. Thanks students for sharing their ideas. Continues reading aloud and stops at the end of page 7 .

10. The instructor observes whether students are coding the text. Asks students to share how they have coded the text so far. If no one has coded the text, tries to think of a way that the information on page seven could be coded, either with “* or !!” For example, thinks aloud saying, "I wonder how the girl feels about being the only one in her class to wear a dupatta. Can you think of a time when you felt different? Uses the sentence-starter "This reminds me of" to share a connection that you have." Listens to and accepts responses. Thanks students for sharing. Reminds them to code the text with * if they have a connection to share. Continues reading to the end of page 14 .

11. The instructor thinks aloud saying, "I think it is interesting that the author chose to have Farah pick a green apple. I am going to code the text with !! because it is interesting for me to think about what Farah has in common with the green apple. Does anyone want to share their thinking about this part of the text?" Listen to and accept responses. Thank students for sharing. Continue reading aloud to the end of page 19.

12. The instructor thinks aloud, saying, "I think I want to re-read this page to be sure that I can understand what is happening. [Re-reads page 19.] When I read about the boy trying to stop Farah it reminded me of a time that I saw students making fun of someone who looked different. It seems like the boy did not like the idea of having a different color apple be part of the apple cider. I am going to code the text with a *." Continues reading aloud to the end of the text, stopping to ask to students to share their thinking when they code the text.

13. The instructor has participant select and read an instructional-level text. Each student in the group reads a different text. Provides students with sticky notes to code their thinking. Provides visual cues to show how to code the text. Rotates through the group and have students read portions of the text aloud. Notes the level of word-reading accuracy demonstrated by participants (number of words read correctly divided by the total number of words read aloud). 
Records the date, the title of the text, the genre of the text, and other relevant information about the lesson on the formative assessment data recording form.

For the next 10 minutes each of you will read from a book in your book box. As you read, use sticky notes to code the text. You can use the symbols ?? !! and * to leave tracks of your thinking. Begin reading silently. I will ask some of you to read aloud to me. Any questions? [Answers questions.] Begin.

14. The instructor poses scripted literal and inferential comprehension questions. Paraphrase participants' responses. Records (+) for on-target responses and (-) for off-target responses on data sheets, along with date, title of text, and genre of text.

15. The instructor asks participant to talk about the text as well as to explain how he or she used the targeted comprehension strategy. Uses scripted prompts, including:

- What problems did you have while reading?

- What did you do to solve problems you had while reading?

- How do you know when you understand text?

16. The instructor, after listening to responses and observing reading behavior, chooses the performance level that best reflects the student's performance (i.e., assigns a rubric score.) Records rubric score and targeted strategy on data sheet.

17. The instructor asks the participant to reflect on his or her goal. Look at the goal you recorded at the beginning of the session. Think about the progress you made toward meeting your goal. Make a note to show whether you met your goal.

18. The instructor has the participant verbally pose questions, make comments, and offer insights by posting to a weblog or by writing in a reader's response notebook. 


\section{Fidelity of Treatment}

Lesson 4 Guided Practice using the Comprehension Strategy with Instructional-Level Text

Date: Total Components: $/ 19$

Observer:

Participant:

Group Size

Day lesson started: Was the lesson completed in one session?

If NO, please indicate where the lesson stopped, section:

Date resumed: Section where lesson began:

Date Completed:

Total \# of components completed successfully

Text Genre

Literal Questions Inferential Questions 1.

2.

1.

2.

3.

4.

5.

3.

4.

5.

Comprehension Rubric Score:

Note on Text Colors

Black = Step by step instructions;

Blue = Teacher's script;

Red $=$ Important reminders. 
Fidelity of Treatment

Lesson 4 Guided Practice using the Comprehension Strategy with Instructional-Level $\underline{\text { Text }}$

1. The instructor has students set goals for the day. The instructor states: "Select a goal to work on today as readers. Record your goal in your reader's response notebook. [Praises students for recording a goal.] Today, my goal is to continue practicing the text coding strategy."

2. The instructor presents the agenda:

- Use TRACK to code Animals That Sting

- Share my thinking about the text

- Reflect on my goal

- Create a kidblog post to share my responses to text

3. The instructor presents instructional-level text. States: "Today, we will read together from a nonfiction story called Animals That Sting by Claire Saxby. This story will teach us how different animals interact with the environment." Has other students in the group read books from their book boxes.

4. The instructor asks questions to activate prior knowledge on the text topic; has participant use mind-mapping applications to create idea webs. States: "Do you know of any animals that sting?" Make a web of ideas." Praises students for contributing ideas.

5. The instructor revisits the comprehension strategies for monitoring for meaning. States: "Today we are going to practice the text coding strategy. This is a strategy that will help us read and comprehend complex literary and informational text." (CCSS. ELALiteracy.CCRA.R.10).

6. The instructor restates the purpose of the comprehension strategies. States: "When we code the text, we leave tracks of our thinking. This strategy helps us to reflect on and react to text. Using TRACK helps us monitor for meaning while we read."

7. The instructor revisits sentence-starters related to the comprehension strategy. Says: "Last time we met I used sentence-starters to talk about the strategy that I was using while thinking aloud about the text." Holds up sentence-starters on sentence strips. "For the strategy ask questions, I started sentences with ..." Has students select the corresponding sentence strip. Provides praise for attempting/achieving the task. "For the strategy "connect" I used the sentence-starter..." Has students select the corresponding sentence strip. Provides praise for attempting/achieving the task. "When I was monitoring my comprehension and realized that what I read did not make sense, I 
used...." Has students select the corresponding sentence strip. Provides praise for attempting/achieving the task.

8. The instructor presents the TRACK mnemonic. Asks, "What key word do we use to remind us how to TRACK our thinking?" Listens to responses, and accepts TRACK. States: "Let's see if we can remember what each of the letters in the word TRACK stand for." Presents reading comprehension strategy cue card. Covers up the card and reveal its contents letter by letter as students collaboratively recall the strategy. Provides praise for each attempt to recall the strategy.

9. The instructor has participant read aloud from instructional level text until reaching a predetermined stopping point. Says, "We are going to read aloud from Animals That Sting. While we read, we will pay attention to what we are thinking. Let's code the text as we read. Do you have any questions about what we are going to do?" Listens and answers any questions. Says, "Please begin reading here." Monitors as participant reads; directs him or her to stop at page 62.

10. The instructor thinks aloud saying, "I think it is interesting that some animals sting for more than one reason. I am going to code the text with !!" Observes whether participant is coding the text also. Says, "Let's continue reading aloud together to the end of page 5."

11. The instructor thinks aloud, saying, "This reminds me how people drink milk or soda through a straw so I am going to code the text with a *."

12. The instructor continues having the participant read aloud. If the participant does not use a sentence-starter or code the text, asks the participant after every second page to share what he or she is thinking. Provides feedback on text coding.

13. The instructor models or provides a prompt for the participant to use fix-up strategies to decode the word tentacles. Stops reading together after page 10.

14. The instructor has non-participants continue to read an instructional-level text. Has the participant finish reading the rest of Animals That Sting aloud. Notes the wordreading accuracy demonstrated by participant (number of words read correctly divided by the total number of words read -there are 71 words from the top of page 11 to the end of the book). Record the date, the title of the text, the genre of the text, and other relevant information about the lesson on the formative assessment data recording form.

15. The instructor poses scripted literal and inferential comprehension questions. Paraphrases participants' responses. Records (+) for on-target responses and (-) for offtarget responses on data sheets, along with date, title of text, and genre of text. 
Literal questions

- What does the word spines mean in this passage?

- What are two reasons that animal might sting?

- When does a stingray use its tail like a whip?

- Where do bees and wasps store their poison sacs?

- Scorpions have poison in Inferential questions

- Which is an appropriate title for this passage? Amazing Animals or What Makes an Animal Sting

- What is this passage trying to explain?

- Which is most likely true about mosquitoes? There are too many of them outside or They sting when they are hungry.

- From this passage, what can you infer about stingrays?

- What can you conclude about animals that sting?

16. The instructor asks the participant to talk about the text as well as to explain how he or she used the targeted comprehension strategy. Uses scripted prompts, including:

- What problems did you have while reading?

- What did you do to solve problems you had while reading?

- How do you know when you understand text?

17. The instructor, after listening to responses and observing reading behavior, chooses the performance level that best reflects the student's performance (i.e., assigns a rubric score.) Records rubric score and targeted strategy on data sheet.

18. The instructor asks the participant to reflect on his or her goal. Says: "Look at the goal you recorded at the beginning of the session. Think about the progress you made toward meeting your goal. Make a note to show whether you met your goal."

19. The instructor has the participant verbally pose questions, make comments, and offer insights by posting to a weblog or by writing in a reader's response notebook. 


\section{Fidelity of Treatment}

Lesson 5: Guided Practice with the Self-Monitoring Procedure

Date:

Total Components:

$/ 24$

Observer:

Participant:

Group Size

Day lesson started:

Was the lesson completed in one session?

If NO, please indicate where the lesson stopped, section:

Date resumed: Section where lesson began:

Date Completed:

Total \# of components completed successfully

Text Genre

Literal Questions

1.

2.

3.

4.

5.

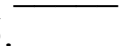

Inferential Questions

1.

2.

3.

4.

5.

Comprehension Rubric Score:

Note on Text Colors

Black = Step by step instructions;

Blue $=$ Teacher's script;

Red $=$ Important reminders. 
Fidelity of Treatment

\section{Lesson 5: Guided Practice with the Self-Monitoring Procedure}

1. The instructor begins by having students set goals for the day. States: "Select a goal to work on today as readers. Record your goal in your reader's response notebook." Praises students for recording a goal. "Today, my goal is to follow four steps to monitor my use of the text coding strategy."

2. The instructor presents the agenda.

Agenda

- Read text from book boxes

- Use the self-monitoring and TRACK procedures

- Share my thinking about the text

- Reflect on my goal

- Create a kidblog post to share my responses to text

3. The instructor presents text written at participant's instructional level. States: "Today, we will read . This story is about "

4. The instructor asks questions to activate prior knowledge on the text topic; has participant use mind-mapping applications to create idea webs. States: "Can you think of ?" Makes a web of ideas. Praises students for contributing ideas to the web.

5. The instructor revisits the comprehension strategies for monitoring for meaning. States: "We will use the text coding strategy as we read. The word TRACK can help us remember to code the text and to monitor for meaning while we read."

6. The instructor states the purpose of the comprehension strategies. States:

"Remember that this strategy helps us read and comprehend complex literary and informational text (CCSS. ELA-Literacy.CCRA.R.10). When we code the text, we leave tracks of our thinking. We use this strategy to reflect on and react to text."

7. The instructor displays the TRACK mnemonic and sentence-starters related to the comprehension strategies. Says, "Last time we met, we used sentence-starters to talk about the strategy that we used while thinking aloud about the text." Hold up sentencestarters on sentence strips. "For the strategy ask questions, we started sentences with ..." Has students select the corresponding sentence strip. Provides praise for attempting/achieving the task. "For the strategy connect we used the sentence-starter..." Has students select the corresponding sentence strip. Provides praise for attempting/achieving the task. "When we monitored comprehension and realized that what we read did not make sense, we used..." Has students select the corresponding sentence strip. Provides praise for attempting/achieving the task. "What key word do we use to remind us how to TRACK our thinking?" Listens to responses, and accepts 
TRACK. "Let's see if we can remember what each of the letters in the word TRACK stand for." Presents reading comprehension strategy cue card. Covers up the card and reveasl its contents letter by letter as students collaboratively recall the strategy. Provides praise for each attempt to recall the strategy.

8. The instructor presents the self-monitoring checklist. States: "Today you will practice following four steps to help you use the TRACK strategy so you can monitor for meaning while you read. Let's look at the self-monitoring procedure card to learn what the four steps are."

9. The instructor explains the purpose of the self-monitoring checklist. States: "This card will help you use the TRACK strategy. You will use the card to make sure that you completed all of the steps involved in coding the text. Coding the text is a strategy that good readers use to comprehend what you read. I will show you how I use the card to monitor how I use the text coding strategy."

10. The instructor has participant read aloud from instructional-level text. Provides prompt, if needed, to check off this step on the checklist. Says, "Open the book and read aloud the first paragraph." Monitors to see whether participant checks off the first step on the checklists. If not, provides a prompt saying, "Check off the first step on the self-monitoring procedure checklist." Asks the participant to read aloud step 2 on the self-monitoring procedure card.

11. The instructor refers to the TRACK strategy card and thinks aloud about the first paragraph, saying

"T means __ [ [ _ _ pave participant supply response] and R means [have participant supply response]. That's good! We are using the

TRACK card to recall the steps of the strategy." Together they check off the second step on the checklist.

12. The instructor asks participant to think aloud about what he or she read in the first paragraph. Provides a model if no response is given. Says, "I am curious about ". [This models using the letter A in track, step 3 on the checklist.] "I am

going to code the text using __ because _____." [This models step 4 on the checklist.]

13. The instructor asks participant to continue reading from the text to a designated stopping point.

14. The instructor asks the participant to think aloud about what he or she read. Provides a model if no response is given. Says, "A stands for ask questions. What does the author mean about ?" I think I am going to code the text using "??" because this is making me wonder. 
15. The instructor asks the participant to continue reading a designated stopping point.

16. The instructor asks participant to think aloud about what he or she read. Provides a model if no response is given. Says, "C stands for connect. This reminds me of . I am going to code the text using because ,'

17. The instructor says, "We used each letter to think about the text. So, I let's check off step three. Also, we coded the text to leave tracks of my thinking, so let's check off step four. Now, let's keep reading."

18. The instructor asks the participant to state the purpose of the self-monitoring procedure. Provides a cue if needed, saying, "The four steps on the self-monitoring procedure card will help you use the TRACK strategy so you can monitor for meaning while you read."

19. The instructor has participant select and read an instructional-level text. Each student in the group reads a different text. Provides students with sticky notes to code their thinking. Provides visual cues to show how to code the text. Rotates through the group and has students read portions of the text aloud. Notes the level of word-reading accuracy demonstrated by participant (number of words read correctly divided by the total number of words read aloud). Records the date, the title of the text, the genre of the text, and other relevant information about the lesson on the formative assessment data recording form.

Says, "For the next 10 minutes each of you will read from a book in your book box. As you read, use sticky notes to code the text. You can use the symbols ??. !! and * to leave tracks of your thinking. Begin reading silently. I will ask some of you to read aloud to me. Any questions?" [Answers questions.] "Begin."

20. The instructor poses scripted literal and inferential comprehension questions. Paraphrases participants' responses. Records (+) for on-target responses and (-) for offtarget responses on data sheets, along with date, title of text, and genre of text.

21. The instructor asks the participant to talk about the text as well as to explain how he or she used the targeted comprehension strategy. Uses scripted prompts, including:

- What problems did you have while reading?

- What did you do to solve problems you had while reading?

- How do you know when you understand text?

22. The instructor, after listening to responses and observing reading behavior, chooses the performance level that best reflects the student's performance (i.e., assigns a rubric score.) Records rubric score and targeted strategy use on data sheet. 
23. The instructor asks participant to reflect on his or her goal. Says: "Look at the goal you recorded at the beginning of the session. Think about the progress you made toward meeting your goal. Make a note to show whether you met your goal."

24. The instructor will has the participant verbally pose questions, make comments, and offer insights by posting to a weblog or by writing in a reader's response notebook. 


\section{Fidelity of Treatment}

Lesson 6 Independent Practice Sessions

Date:

Total Components:

$/ 16$

Observer:

Participant:

Group Size

Day lesson started:

Was the lesson completed in one session?

If $\mathrm{NO}$, please indicate where the lesson stopped, section:

Date resumed: Section where lesson began:

Date Completed:

Total \# of components completed successfully

Text Genre

Literal Questions Inferential Questions

1.

1.

$2 . \quad 2$

$3 . \quad 3$

4.4 . 4.

$5 . \quad 5$.

Comprehension Rubric Score:

Note on Text Colors

Black = Step by step instructions;

Blue $=$ Teacher's script;

Red $=$ Important reminders. 
Fidelity of Treatment

\section{Lesson 6: Independent Practice Sessions}

1. The instructor begins by having students set goals for the day. States: "Select a goal to work on today as readers. Record your goal in your reader's response notebook." Praises students for recording a goal.

2. The instructor presents the agenda.

Agenda:

- Read text from book boxes

- Use the self-monitoring and TRACK procedures

- Share my thinking about the text

- Reflect on my goal

- Create a kidblog post to share my responses to text

3. The instructor presents a text written at the participant's instructional level. States: "Today, we will read books from our book boxes."

4. The instructor asks questions to activate prior knowledge on the text topic; has participant use mind-mapping applications to create idea webs. States: "Preview the text you select and create a web of ideas to activate your background knowledge."

5. The instructor asks participant to state the purpose of the comprehension strategy. Says, "Be sure to use the text coding strategy as you read. How does this strategy help us when we read?" Listens to responses. Provides a cue, if needed: "The word TRACK can help us remember to code the text and to monitor for meaning while we read."

6. The instructor displays sentence-starters related to the comprehension strategies. Says, "While you read you can use our sentence-starters to think about the text." Holds up sentence-starters on sentence strips.

7. The instructor displays the TRACK mnemonic. Says, "What key word do we use to remind us how to TRACK our thinking?" Listens to responses, and accepts TRACK. "What does each letters in the word TRACK stand for?" Presents reading comprehension strategy cue card. Covers up the card and reveals its contents letter by letter as students collaboratively recall the strategy. Provide praises for each attempt to recall the strategy.

8. The instructor presents the self-monitoring checklist. States: "Today you will follow the four steps on the self-monitoring procedure card as you use the TRACK strategy. Let's look at the self-monitoring procedure card to learn what the four steps are." 
9. The instructor provides the participant with sticky notes. Directs participant to determine how and why to code the text and to leave tracks of thinking. Says, "Use the card to make sure that you complete all of the steps involved in coding the text." Passes out sticky notes. Asks students how and why to code the text. Provides a cue if needed, saying, "The four steps on the self-monitoring procedure card will help you use the TRACK strategy so you can monitor for meaning while you read."

10. The instructor has participant select and read an instructional-level text. Each student in the group reads a different text. Provides students with sticky notes to code their thinking. Provides visual cues to show how to code the text. Rotates through the group and has students read portions of the text aloud. Notes the level of word-reading accuracy demonstrated by participants (number of words read correctly divided by the total number of words read aloud). Records the date, the title of the text, the genre of the text, and other relevant information about the lesson on the formative assessment data recording form.

Says, "For the next 10 minutes each of you will read from a book in your book box. As you read, use sticky notes to code the text. You can use the symbols ??.!! and * to leave tracks of your thinking. Begin reading silently. I will ask some of you to read aloud to me. Any questions?" [Answers questions.] "Begin."

11. The instructor poses scripted literal and inferential comprehension questions. Paraphrases participant's responses. Records (+) for on-target responses and (-) for offtarget responses on data sheets, along with date, title of text, and genre of text.

12. The instructor asks the participant to talk about the text as well as to explain how he or she used the targeted comprehension strategy. Uses scripted prompts, including:

- What problems did you have while reading?

- What did you do to solve problems you had while reading?

- How do you know when you understand text?

13. The instructor, after listening to responses and observing reading behavior, chooses the performance level that best reflects the student's performance (i.e., assign a rubric score.) Records rubric score and targeted strategy on data sheet.

14. The instructor asks the participant to reflect on his or her goal. Says: "Look at the goal you recorded at the beginning of the session. Think about the progress you made toward meeting your goal. Make a note to show whether you met your goal."

15. The instructor has the participant verbally pose questions, make comments, and offer insights by posting to a weblog or by writing in a reader's response notebook. 
16. After participants complete 3 independent practice lessons, the instructor has them video record and narrate use of the comprehension strategy with the self-monitoring procedure. 


\section{APPENDIX K}

\section{EXPLICIT INSTRUCTION CHECKLIST}

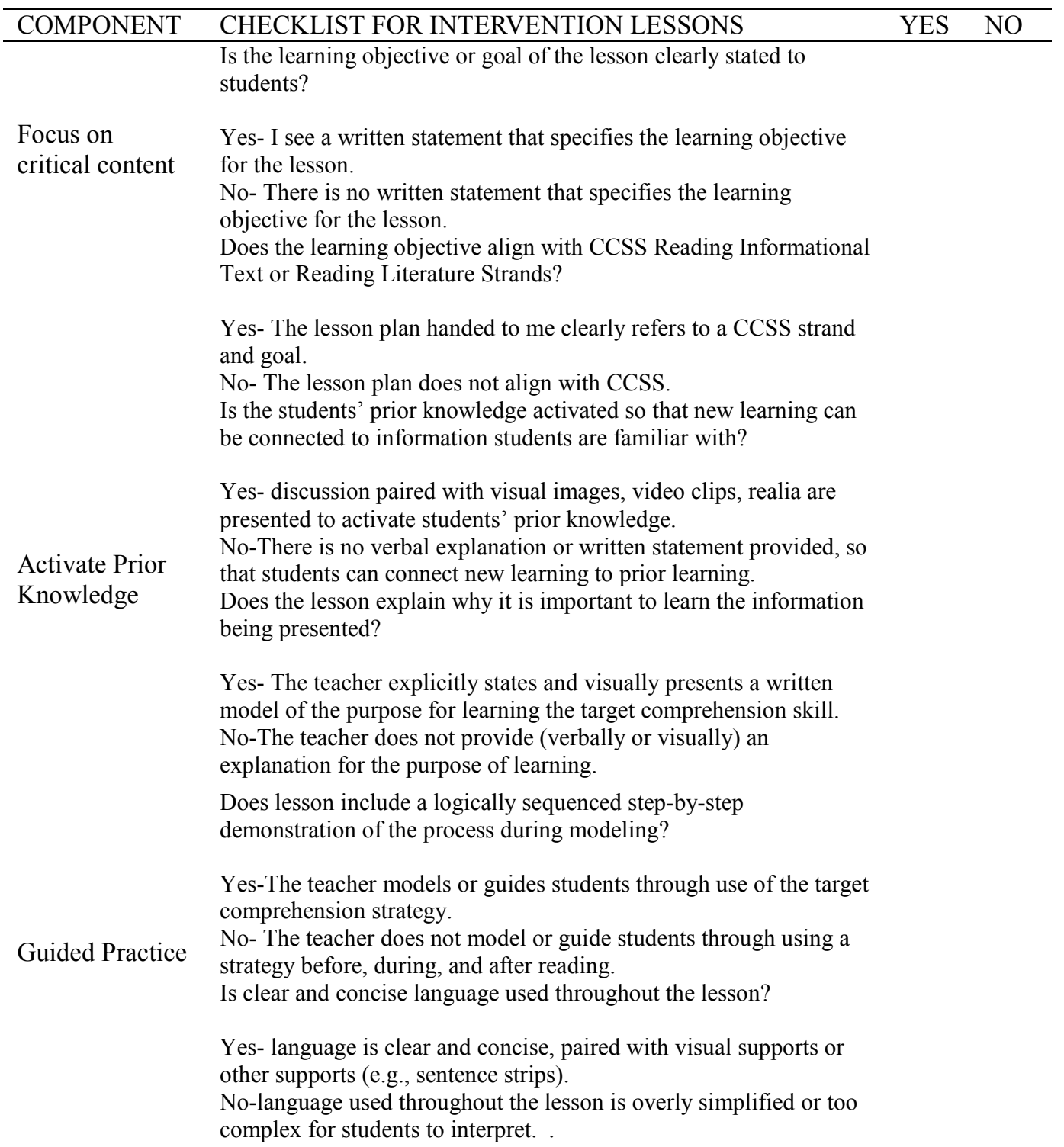

(Table Continue) 
Are demonstrations or realia used during the lesson?

Yes-The lesson includes at least one demonstration of the target skill being modeled through a think-aloud by the teacher. NoThere are no demonstrations used during the lesson and realia is not used during the lesson when it could be used to clarify a concept.

Are the teacher's inner thoughts (think-alouds) shared?

Yes- the teacher models use of a comprehension strategy through a think-aloud.

No- the teacher does not model use of a comprehension strategy through a thin- aloud.

Does the lesson offer frequent checking for understanding?

Yes-informal assessments are used (entrance slips, exit slips, thumbs up, thumbs down, visually scanning the group to look for nonverbal cues from students).

No- the teacher does not attempt to recognize signs that students are confused or in need of support.

Does the lesson require frequent oral responses?

Yes: the following were used_choral_ think-pair-share _think pair, write, share _response cards _ slate boards_ hand signals

Checks for Understanding gestures _actions

No: none of the above were used Are student responses monitored carefully?

Yes-the teacher is actively listening, probing, and reframing responses.

No-the teacher is superficially acknowledging or failing to acknowledge responses.

Is immediate affirmative and corrective feedback provided?

Yes-there is more positive than negative feedback provided; important misconceptions are clarified.

No-the ratio of positive to negative feedback is a concern; misconceptions are allowed to go unchecked.

Have all the skills needed for independent practice been taught so students can experience success during independent practice?

Independent Yes- important information was modeled and practiced.

Practice

Goal Setting
No-some key steps were missing.

Are initial practice attempts provided and monitored?

Yes-initial practice attempts are provided and monitored. No-initial practice attempts were not provided or not monitored. Are students given the opportunity to set their own goals based on their achievement?

Yes-students set their own goals based on their achievement. No-There is no evidence of students setting their own goals. 


\section{APPENDIX L \\ SCRIPTED INTERVENTION LESSON PLANS}

Lesson 1: Modeling the Comprehension Strategy

Materials:

- Reader's response notebooks

- Mentor Text (i.e., A Day's Work by Eve Bunting)

- Sentence-starters (This reminds me of, I am confused about, I wonder why)

- TRACK mnemonic

- Sticky notes for coding the text

- Selection of instructional-level texts for each participant

- Scripted comprehension check questions to accompany each text

- Comprehension thinking strategy rubric

- Data recording forms

Note on Text Colors

Black = Step by step instructions;

Blue $=$ Teacher's script;

Red $=$ Important reminders.

Purpose: Model using the TRACK mnemonic to apply comprehension strategies while reading.

1. The lesson will begin by setting goals for the day. State: "Select a goal to work on today as readers. Record your goal in your reader's response notebook. [Praise students for recording a goal.] Today, my goal is to leave tracks of my thinking while I read. During the next few weeks, you learn how tracking your thinking can help you to better understand the texts that you read." 
2 Present the agenda:

- Listen to the story A Day's Work

- Learn how to use TRACK to code the text

- Read text from book boxes

- Share my thinking about the text

- Reflect on my goal

- Create a kidblog post to share my responses to text

3. Present mentor text. State: “Today, I will read aloud from a story called A Day's Work by Eve Bunting. This story shows us how honesty and integrity are smart choices in any situation."

4. Ask questions to activate prior knowledge on the text topic; have participant use mind-mapping applications to create idea webs. State: Have you ever heard the expression honesty is the best policy? There are many ways to solve a problem. Honesty is one of the best solutions. What do you think it means if you tell someone about a problem, such as forgetting your homework, and they say honesty is the best policy? How could honesty solve the problem of not having your homework? What are some other examples of situations where honesty is the best policy?" [Make a web of ideas. Praise students for contributing ideas.]

5. Introduce the comprehension strategies for monitoring for meaning. State: "Today I will show you a strategy called text coding. Text coding is a strategy that readers can use to reflect on and react to what they read. When I code the text, I leave tracks of my thinking. This means that you will be able to see what I was thinking while I was reading a text."

6. Explain the purpose of the comprehension strategies. State: "Nothing is more important during reading than the reader's thinking. I will read the book A Day's Work with you today because it makes me think about so many things. When readers pay attention and think about the words and ideas in text, they have an inner conversation with the text. It is a quiet conversation that happens only in the reader's head. Today, when I code the text, I will leave tracks so that you can follow my thinking."

7. Explain sentence-starters and explain the TRACK mnemonic. Say: "I will use the word TRACK to remind me of the steps I need to follow as I code the text." Display the TRACK mnemonic by uncovering one letter at a time. 
Reading Strategy: Code the text to keep TRACK of your thinking.

Think about what I am reading.

React to the text.

Ask questions.

Connect.

Keep track of your thinking.

Continue reading script below:

a. The first letter in the word TRACK is "T." The letter "T" reminds me to think about what I am reading. Today, I will show you how I pay attention to my thoughts as I read. For example, I might read something that confuses me and think, "Huh, I don't get this part." I might read something that tells me new information and think, "Wow, I never knew that before." The letter "T" reminds me to think about what I am reading read so I can have an inner conversation with the text. Do you like the phrase "Think about what I am reading."? Or do you prefer, "Think while I read."?

b. The second letter in the word TRACK is "R." The letter " $\mathrm{R}$ " reminds me to react to the text. It is not enough to just read and think about the text. I also have to react and explore my thinking. This means that when I notice my thinking, I also do something about it. For example, if I notice that I am confused by a word that is hard to read, I will do something about it. I might read ahead and use context clues to try to figure out what the word means or I might circle the word and decide to look it up in the dictionary. Today, when I think aloud about what I am reading you might know that I am going to react to my thinking if you hear me say, "I am confused about ." [Hold up sentence-starter written on a sentence strip.] The letter "R" reminds me to do more than just read and think about the text-it tells me to react. Do you like the phrase "React to the text" or do you prefer "Respond to the text"?

c. The third letter in the word TRACK is "A." The letter "A" reminds me to ask questions. Asking questions and wondering about text is part of what good readers do. Some questions that readers ask can be answered easily in few words. Others are big questions that have long, involved answers. And other questions can't be answered at all. Today, when I show you how I ask questions while I read, you might hear me say, "I wonder why the author chose to ." [Hold up sentence-starter written on a sentence strip.] The letter "A" reminds me that it is important 
to ask questions while I read. Do you like the phrase "ask questions" or do you prefer "ask a question to the author?"

d. The fourth letter in the word TRACK is " $\mathrm{C}$." The letter " $\mathrm{C}$ " reminds me to connect to what I am reading. Good readers make connections between the books they read and their own lives. Today, I will show you how I make connections while reading. You will now that I am going to connect to what I am reading when you hear me say, "This reminds me of

." [Hold up sentence-starter written on a sentence strip.] The letter "C" reminds me to connect my own knowledge and experiences to what I am reading. Do you like the phrase "connect" or do you prefer "connect to the text?"

e. The final letter in the word TRACK is "K." The letter "K" reminds me to keep track of my thinking. To keep track of my thinking, I will code the text. I will use symbols to show what I am thinking. I will write a symbol on a sticky note and place the sticky note on the page of the text that made me react. Do you like the phrase "Keep track of my thinking" or do you prefer "Keep track of what I think while I read."? Some of the symbols I might use are:

iv. "??" to show that I am confused or that I am wondering something

v. " "!?" to show that I read something that I think is interesting

vi. “*” to show that I made a connection to something I already know

8. Read aloud from mentor text until reaching a predetermined stopping point. State: "I am going to read aloud from A Day's Work. While I read, I will think aloud and I will code the text to leave tracks of my thinking. Your job is to listen to the story, listen to my thinking, and notice how I code the text." Begin reading aloud the first page of the story. Stop on page two mid-way through the page before the paragraph that begins with "Francisco swallowed..."

9. Think aloud by using the sentence-starters that accompany comprehension strategies. Hold up sentence-starter. "I am confused about why Francisco is standing with his grandfather in a parking lot on a Saturday morning."

10. Model using sticky notes to code the text and to leave tracks of thinking while verbally explaining how and why to code text. State: "What I am reading does not make sense to me yet, I am going to code the text with "??" symbols to remind myself that I can use strategies to figure this out. I will read ahead and see if I can figure out why Francisco is waiting with his grandfather." Continue reading and stop at the bottom of page 6 after the sentence that ends "skinny as an old tree." "Now that I have read ahead, I 
figured out that Francisco was there to help his grandfather find work for the day. I wonder why the author chose to describe the grandfather using the simile "as skinny as an old tree." Code the text with ?? "When a tree gets very old, its branches become thin. It makes me think that maybe the author wants me to think that Francisco's grandfather is delicate or fragile, easily broken."

11. Continue reading aloud from mentor text, stopping to think aloud. Read aloud; stop at the bottom of page 10. State: "It seems like Francisco is very brave. The author described how Francisco pushed a tough guy out of the way. I think the tough guy was going to try to steal the job from Francisco and his grandfather." Hold up sentencestarter. "This reminds me of how I can surprise myself by acting bravely when I really want to accomplish something" Code text with a * and continue reading aloud and stop in the middle of page 20 after the sentence, "Francisco and his grandfather shook hands." Say: "I can tell that Francisco and his grandfather are proud of all the hard work they accomplished. When the author wrote that Francisco and his grandfather shook hands, it reminded me of what I see a team do after playing hard in a game." Code the text with a * symbol.

12. While reading, model "fix up" strategies at word level, text level, and schema level (e.g., decode difficult words, re-read, read ahead, ask questions, or make connections). Continue reading aloud from the text and stop near the end of page 22 after reading aloud the sentence, "You took out my young ice plants." Say to students: I am confused. I think I need to read that sentence again. "You took out my young- iceplants." I am not sure what an ice plant is. Code text with ?? symbols. It seems like Ben is not happy about the work that Francisco and his grandfather did. I am going to read on to see if I can tell why Ben is upset. Continue reading to the end of the page. Okay, Ben is definitely upset. The author wrote that Ben slammed his Lakers hat against the van. This reminds me of what I have seen people do when they are really angry. But, I am still not sure why Ben is so angry. Code text with * and ??; continue reading aloud through the top of page 30 , to the sentence that ends, "we take the pay tomorrow, when we finish." I am starting to think that maybe I was off-track when I thought that the author compared the grandfather to an old tree to make me think that he is fragile. Reading about how abuelo handled this problem made me change my mind. He showed strength and integrity by not taking the money and by offering to come back to fix the mistake. Maybe old trees are stronger than I thought. Code the text with !! to show that I read something that made me think differently; continue reading to the end of the story. 
13. Have participant select and read an instructional-level text.

Each student in the group will read a different text. Provide students with sticky notes to code their thinking. Provide visual cues to show how to code the text. Rotate through the group and have students read portions of the text aloud. Note the level of word-reading accuracy demonstrated by participants (number of words read correctly divided by the total number of words read aloud). Record the date, the title of the text, the genre of the text, and other relevant information about the lesson on the formative assessment data recording form.

Say, "For the next 10 minutes each of you will read from a book in your book box. As you read, use sticky notes to code the text. You can use the symbols ??.!! and * to leave tracks of your thinking. Begin reading silently. I will ask some of you to read aloud to me. Any questions? [Answer questions.] Begin.”

14. Pose scripted literal and inferential comprehension questions. Paraphrase participants' responses. Record (+) for on-target responses and (-) for off-target responses on data sheets, along with date, title of text, and genre of text.

15. Ask participant to talk about the text as well as to explain how he or she used the targeted comprehension strategy. Use scripted prompts, including:

- What problems did you have while reading?

- What did you do to solve problems you had while reading?

- How do you know when you understand text?

16. After listening to responses and observing reading behavior, choose the performance level that best reflects the student's performance (i.e., assign a rubric score.) Record rubric score and targeted strategy on data sheet.

17. Ask participant to reflect on his or her goal. Say: "Look at the goal you recorded at the beginning of the session. Think about the progress you made toward meeting your goal. Make a note to show whether you met your goal."

18. Have participant verbally pose questions, make comments, and offer insights by posting to a weblog or by writing in a reader's response notebook. 
Lesson 2: Modeling the self-monitoring procedure

Materials:

- Reader's response notebooks

- Mentor Text (i.e., The Day of Ahmed's Secret by Ted Lewin)

- Sentence-starters (This reminds me of, I am confused about)

- TRACK mnemonic

- Self-monitoring procedure card

- Sticky notes for coding the text

- Selection of instructional-level texts for each participant

- Scripted comprehension check questions to accompany each text

- Comprehension thinking strategy rubric

- Data recording forms

Note on Text Colors

Black $=$ Step by step instructions;

Blue $=$ Teacher's script;

Red $=$ Important reminders .

Purpose: Demonstrate how to use the self-monitoring procedure while reading and coding text.

1. The lesson will begin by setting goals for the day. State: "Select a goal to work on today as readers. Record your goal in your reader's response notebook. [Praise students for recording a goal.] "Today, my goal is to monitor how I use the text coding strategy."

Agenda:

- Listen to the story The Day of Ahmed's Secret

- Read text from book boxes

- Share my thinking about the text

- Reflect on my goal

- Create a kidblog post to share my responses to text

2. Present mentor text. State: "Today, I will read aloud from a story called The Day of Ahmed's Secret by Ted Lewin. This story is about a boy who waits very patiently all day for a special moment.

3. Ask questions to activate prior knowledge on the text topic; have participant use mind-mapping applications to create idea webs. State: Can you think of something that you have waited patiently for?" Make a web of ideas. Praise students for contributing ideas to the web. 
4. Revisit the comprehension strategies for monitoring for meaning. State: "We will use the text coding strategy as we read. The word TRACK can help us remember to code the text and to monitor for meaning while we read."

5. State the purpose of the comprehension strategies. State: "Remember that this strategy helps us read and comprehend complex literary and informational text (CCSS. ELA-Literacy.CCRA.R.10). When we code the text, we leave tracks of our thinking. We use this strategy to reflect on and react to text."

6. Display the TRACK mnemonic and sentence-starters related to the comprehension strategies. Say, "Last time we met, we used sentence-starters to talk about the strategy that we used while thinking aloud about the text." Hold up sentence-starters on sentence strips. "For the strategy ask questions, we started sentences with ..." Have students select the corresponding sentence strip. Provide praise for attempting/achieving the task. "For the strategy connect we used the sentence-starter..." Have students select the corresponding sentence strip. Provide praise for attempting/achieving the task. "When we monitored comprehension and realized that what we read did not make sense, we used...." Have students select the corresponding sentence strip. Provide praise for attempting/achieving the task. "What key word do we use to remind us how to TRACK our thinking?" Listen to responses, and accept TRACK. "Let's see if we can remember what each of the letters in the word TRACK stand for." Present reading comprehension strategy cue card. Cover up the card and reveal its contents letter by letter as students collaboratively recall the strategy. Provide praise for each attempt to recall the strategy.

Reading Strategy: Code the text to keep TRACK of your thinking.

Think about what I am reading.

React to the text.

Ask questions.

Connect.

Keep track of your thinking.

7. Present the self-monitoring checklist. State: "Today you will learn four steps to help you use the TRACK strategy so you can monitor for meaning while you read. Let's look at the self-monitoring procedure card to learn what the four steps are." 
$\square$ I read the paragraph.

$\square$ I used the prompt card to recall the strategy steps.

$\square$ I applied the strategy to monitor for meaning.

$\square$ I coded the text to leave tracks of my thinking.

8. Explain the purpose of the self-monitoring checklist. State: "This card will help you use the TRACK strategy. You will use the card to make sure that you completed all of the steps involved in coding the text. Coding the text is a strategy that good readers use to comprehend what you read. I will show you how I use the card to monitor how I use the text coding strategy."

9. Read aloud from mentor text. Open the book The Day of Ahmed's Secret and read aloud the first paragraph (which happens to be only one sentence long).

10. Model checking off this step on the checklist. Check off the first step on the selfmonitoring procedure checklist.

11. Read aloud step 2 on the self-monitoring procedure card. Refer to the TRACK strategy card and think aloud about the first paragraph, saying:

$\mathrm{T}$ means think while reading and $\mathrm{R}$ means react to the text. I am curious about what his secret could be. He says the secret will be like a friend to him. A stands for ask questions. What does the author mean about the secret being like his friend? I think this means he is going to keep it very close to him and not give anyone any hints about what it could be. $\mathrm{C}$ stands for connect. This reminds me of how I feel when I know about something (like a surprise party) that I need to keep a secret. I am going to code the text "'!" because I like the way the author compared having a secret to having a friend. I used the prompt card to recall the steps for TRACK, so I will check off step two. I used each letter to think about the text. So, I will check off step three on the checklist. I coded the text to leave tracks of my thinking, so I will check off step four. Now, I am going to keep reading the story until I reach a point where I want to ask questions or make connections. Continue reading the story and modeling the self-monitoring procedure.

12. Restate the purpose of the self-monitoring procedure. Say, "The four steps on the self-monitoring procedure card will help you use the TRACK strategy so you can monitor for meaning while you read." 
13. Have participant select and read an instructional-level text. Each student in the group will read a different text. Provide students with sticky notes to code their thinking. Provide visual cues to show how to code the text. Rotate through the group and have students read portions of the text aloud. Note the level of word-reading accuracy demonstrated by participants (number of words read correctly divided by the total number of words read aloud). Record the date, the title of the text, the genre of the text, and other relevant information about the lesson on the formative assessment data recording form.

Say, "For the next 10 minutes each of you will read from a book in your book box. As you read, use sticky notes to code the text. You can use the symbols ??. !! and * to leave tracks of your thinking. Begin reading silently. I will ask some of you to read aloud to me. Any questions? [Answer questions.] Begin."

14. Pose scripted literal and inferential comprehension questions. Paraphrase participants' responses. Record (+) for on-target responses and (-) for off-target responses on data sheets, along with date, title of text, and genre of text.

15. Ask participant to talk about the text as well as to explain how he or she used the targeted comprehension strategy. Use scripted prompts, including:

- What problems did you have while reading?

- What did you do to solve problems you had while reading?

- How do you know when you understand text?

16. After listening to responses and observing reading behavior, choose the performance level that best reflects the student's performance (i.e., assign a rubric score.) Record rubric score and targeted strategy on data sheet.

17. Ask participant to reflect on his or her goal. Say: "Look at the goal you recorded at the beginning of the session. Think about the progress you made toward meeting your goal. Make a note to show whether you met your goal."

18. Have participant verbally pose questions, make comments, and offer insights by posting to a weblog or by writing in a reader's response notebook. 
Lesson 3 Guided Practice with the Comprehension Strategy using Mentor Text

Materials:

- Reader's response notebooks

- Mentor Text (i.e., One Green Apple by Eve Bunting)

- Sentence-starters (This reminds me of, I am confused about, I wonder why)

- TRACK mnemonic

- Sticky notes for coding the text

- Selection of instructional-level texts for each participant

- Scripted comprehension check questions to accompany each text

- Comprehension thinking strategy rubric

- Data recording forms

- Visual cues of symbols used to code text

Note on Text Colors

Black $=$ Step by step instructions;

Blue $=$ Teacher's script;

Red $=$ Important reminders.

Purpose: Provide the opportunity for students to practice and receive feedback on using the TRACK mnemonic to monitor for meaning while reading mentor text and instructional-level text.

1. The lesson will begin by setting goals for the day. State: "Select a goal to work on today as readers. Record your goal in your reader's response notebook. [Praise students for recording a goal.] Today, my goal is to continue practicing the text coding strategy."

2. Agenda:

- Listen to the story One Green Apple

- Use TRACK to code the text

- Read text from book boxes

- Share my thinking about the text

- Reflect on my goal

- Create a kidblog post to share my responses to text

3. Present mentor text. State: "Today, I will read aloud from a story called One Green Apple by Eve Bunting. This story will remind us of what we learned in about in our Second Step Lesson on empathy. Does anyone remember what empathy means?" Listen to ideas. Provide verbal praise for sharing responses. 
4. Ask questions to activate prior knowledge on the text topic; have participant use mind-mapping applications to create idea webs. State: "How could we show empathy to a new student who joins our class but does not speak English yet? Make a web of ideas." Praise students for contributing ideas.

5. Revisit the comprehension strategies for monitoring for meaning. State: "Today we are going to practice the text coding strategy. This is a strategy that will help us read and comprehend complex literary and informational text." (CCSS. ELALiteracy.CCRA.R.10).

6. Restate the purpose of the comprehension strategies. State: "When we code the text, we leave tracks of our thinking. This strategy helps us to reflect on and react to text. Using TRACK helps us monitor for meaning while we read."

7. Revisit sentence-starters related to the comprehension strategy. Say: "Last time we met I used sentence-starters to talk about the strategy that I was using while thinking aloud about the text." Hold up sentence-starters on sentence strips. "For the strategy ask questions, I started sentences with ..." Have students select the corresponding sentence strip. Provide praise for attempting/achieving the task. "For the strategy "connect" I used the sentence-starter..." Have students select the corresponding sentence strip. Provide praise for attempting/achieving the task. "When I was monitoring my comprehension and realized that what I read did not make sense, I used...." Have students select the corresponding sentence strip. Provide praise for attempting/achieving the task.

8. Present TRACK mnemonic. Ask, "What key word do we use to remind us how to TRACK our thinking?" Listen to responses, and accept TRACK. State: "Let's see if we can remember what each of the letters in the word TRACK stand for." Present reading comprehension strategy cue card. Cover up the card and reveal its contents letter by letter as students collaboratively recall the strategy. Provide praise for each attempt to recall the strategy.

9. Read aloud from mentor text until reaching a predetermined stopping point. Say, "I am going to read aloud from One Green Apple. While I read, your job is to listen and think. Follow along in your copy of this story so that you can code the text. At some points, I will stop reading so that we can share our thinking. What is your task?" Listen to responses. Say, "Let's begin." Start reading and stop at page 6. Think aloud saying, "I wonder what the author means when she writes that the students looked at the girl coldly. Why do you think some kids would look at her like that?" Listen to and accept responses. Thank students for sharing their ideas. Continue reading aloud and stop at the end of page 7. 
10. Observe whether students are coding the text. Ask students to share how they have coded the text so far. If no one has coded the text, try to think of a way that the information on page seven could be coded, either with “* or !!” For example, think aloud saying, "I wonder how the girl feels about being the only one in her class to wear a dupatta. Can you think of a time when you felt different? Use the sentence-starter "This reminds me of" to share a connection that you have." Listen to and accept responses. Thank students for sharing. Remind them to code the text with * if they have a connection to share. Continue reading to the end of page 14.

11. Think aloud saying, "I think it is interesting that the author chose to have Farah pick a green apple. I am going to code the text with !! because it is interesting for me to think about what Farah has in common with the green apple. Does anyone want to share their thinking about this part of the text?" Listen to and accept responses. Thank students for sharing. Continue reading aloud to the end of page 19.

12. Think aloud, saying, "I think I want to re-read this page to be sure that I can understand what is happening. [Re-read page 19.] When I read about the boy trying to stop Farah it reminded me of a time that I saw students making fun of someone who looked different. It seems like the boy did not like the idea of having a different color apple be part of the apple cider. I am going to code the text with a *." Continue reading aloud to the end of the text, stopping to ask to students to share their thinking when they code the text.

13. Have participant select and read an instructional-level text. Each student in the group will read a different text. Provide students with sticky notes to code their thinking. Provide visual cues to show how to code the text. Rotate through the group and have students read portions of the text aloud. Note the level of word-reading accuracy demonstrated by participants (number of words read correctly divided by the total number of words read aloud). Record the date, the title of the text, the genre of the text, and other relevant information about the lesson on the formative assessment data recording form.

Say, "For the next 10 minutes each of you will read from a book in your book box. As you read, use sticky notes to code the text. You can use the symbols ??. !! and * to leave tracks of your thinking. Begin reading silently. I will ask some of you to read aloud to me. Any questions? [Answer questions.] Begin.”

14. Pose scripted literal and inferential comprehension questions. Paraphrase participants' responses. Record (+) for on-target responses and (-) for off-target responses on data sheets, along with date, title of text, and genre of text. 
15. Ask participant to talk about the text as well as to explain how he or she used the targeted comprehension strategy. Use scripted prompts, including:

- What problems did you have while reading?

- What did you do to solve problems you had while reading?

- How do you know when you understand text?

16. After listening to responses and observing reading behavior, choose the performance level that best reflects the student's performance (i.e., assign a rubric score.) Record rubric score and targeted strategy on data sheet.

17. Ask participant to reflect on his or her goal. Say: "Look at the goal you recorded at the beginning of the session. Think about the progress you made toward meeting your goal. Make a note to show whether you met your goal."

18. Have participant verbally pose questions, make comments, and offer insights by posting to a weblog or by writing in a reader's response notebook. 
Lesson 4 Guided Practice using the Comprehension Strategy with Instructional-level Text Materials:

- Reader's response notebooks

- Instructional-level text (e.g., Animals That Sting by Claire Saxby)

- Sentence-starters (This reminds me of, I am confused about)

- TRACK mnemonic

- Self-monitoring procedure card

- Sticky notes for coding the text

- Selection of instructional-level texts for each participant

- Scripted comprehension check questions to accompany each text

- Comprehension thinking strategy rubric

- Data recording forms

\section{$\underline{\text { Note on Text Colors }}$}

Black $=$ Step by step instructions;

Blue $=$ Teacher's script;

Red $=$ Important reminders

Purpose: Provide the opportunity for students to practice and receive feedback on using the TRACK mnemonic to monitor for meaning while reading instructional-level text.

1. The lesson will begin by setting goals for the day. State: "Select a goal to work on today as readers. Record your goal in your reader's response notebook. [Praise students for recording a goal.] Today, my goal is to continue practicing the text coding strategy."

2. Agenda:

- Use TRACK to code Animals That Sting

- Share my thinking about the text

- Reflect on my goal

- Create a kidblog post to share my responses to text

3. Present instructional-level text. State: "Today, we will read together from a nonfiction story called Animals That Sting by Claire Saxby. This story will teach us how different animals interact with the environment." Have other students in the group read books from their book boxes.

4. Ask questions to activate prior knowledge on the text topic; have participant use mind-mapping applications to create idea webs. State: "Do you know of any animals that sting?" Make a web of ideas." Praise students for contributing ideas. 
5. Revisit the comprehension strategies for monitoring for meaning. State: "Today we are going to practice the text coding strategy. This is a strategy that will help us read and comprehend complex literary and informational text." (CCSS. ELA-

Literacy.CCRA.R.10).

6. Restate the purpose of the comprehension strategies. State: "When we code the text, we leave tracks of our thinking. This strategy helps us to reflect on and react to text. Using TRACK helps us monitor for meaning while we read."

7. Revisit sentence-starters related to the comprehension strategy. Say: "Last time we met I used sentence-starters to talk about the strategy that I was using while thinking aloud about the text." Hold up sentence-starters on sentence strips. "For the strategy ask questions, I started sentences with ..." Have students select the corresponding sentence strip. Provide praise for attempting/achieving the task. "For the strategy "connect" I used the sentence-starter..." Have students select the corresponding sentence strip. Provide praise for attempting/achieving the task. "When I was monitoring my comprehension and realized that what I read did not make sense, I used..." Have students select the corresponding sentence strip. Provide praise for attempting/achieving the task.

8. Present TRACK mnemonic. Ask, "What key word do we use to remind us how to TRACK our thinking?" Listen to responses, and accept TRACK. State: "Let's see if we can remember what each of the letters in the word TRACK stand for." Present reading comprehension strategy cue card. Cover up the card and reveal its contents letter by letter as students collaboratively recall the strategy. Provide praise for each attempt to recall the strategy.

9. Have participant read aloud from instructional level text until reaching a predetermined stopping point. Say, "We are going to read aloud from Animals That Sting. While we read, we will pay attention to what we are thinking. Let's code the text as we read. Do you have any questions about what we are going to do?" Listen and answer any questions. Say, "Please begin reading here." Monitor as participant reads; direct him or her to stop at page 62 .

10. Think aloud saying, "I think it is interesting that some animals sting for more than one reason. I am going to code the text with !!" Observe whether participant is coding the text also. Say, "Let's continue reading aloud together to the end of page 5."

11. Think aloud, saying, "This reminds me how people drink milk or soda through a straw so I am going to code the text with a *." 
12. Continue having the participant read aloud to you. If the participant does not use a sentence-starter or code the text, ask the participant after every second page to share what he or she is thinking. Provide feedback on text coding.

13. On page 10 consider modeling or prompting participant to use fix-up strategies to decode the word tentacles. Stop reading together after page 10 .

14. Have non-participants continue to read an instructional-level text. Have the participant finish reading the rest of Animals That Sting aloud to you. Note the wordreading accuracy demonstrated by participant (number of words read correctly divided by the total number of words read -there are 71 words from the top of page 11 to the end of the book). Record the date, the title of the text, the genre of the text, and other relevant information about the lesson on the formative assessment data recording form.

15. Pose scripted literal and inferential comprehension questions. Paraphrase participants' responses. Record (+) for on-target responses and (-) for off-target responses on data sheets, along with date, title of text, and genre of text.

Literal questions

- What does the word spines probably spines mean in this passage?

- What are two reasons that animal might sting?

- When does a stingray use its tail like a whip?

- Where do bees and wasps store their poison sacs?

- Scorpions have poison in

Inferential questions

- Which is an appropriate title for this passage? Amazing Animals or What Makes an Animal Sting

- What is this passage trying to explain?

- Which is most likely true about mosquitoes? There are too many of them outside or They sting when they are hungry.

- $\quad$ From this passage, what can you infer about stingrays?

- What can you conclude about animals that sting?

16. Ask participant to talk about the text as well as to explain how he or she used the targeted comprehension strategy. Use scripted prompts, including:

- What problems did you have while reading?

- What did you do to solve problems you had while reading?

- How do you know when you understand text?

17. After listening to responses and observing reading behavior, choose the performance level that best reflects the student's performance (i.e., assign a rubric score.) Record rubric score and targeted strategy on data sheet. 
18. Ask participant to reflect on his or her goal. Say: "Look at the goal you recorded at the beginning of the session. Think about the progress you made toward meeting your goal. Make a note to show whether you met your goal."

19. Have participant verbally pose questions, make comments, and offer insights by posting to a weblog or by writing in a reader's response notebook. 
Lesson 5 Guided Practice with the Self-Monitoring Procedure

Materials:

- Reader's response notebooks

- Sentence-starters (This reminds me of, I am confused about)

- TRACK mnemonic

- Self-monitoring procedure card

- Sticky notes for coding the text

- Selection of instructional-level texts for each participant

- Scripted comprehension check questions to accompany each text

- Comprehension thinking strategy rubric

- Data recording forms

Note on Text Colors

Black $=$ Step by step instructions;

Blue $=$ Teacher's script;

Red $=$ Important reminders.

Purpose: Provide an opportunity for participants to practice and receive feedback on using the self-monitoring procedure while reading and coding instructional-level text.

1. The lesson will begin by setting goals for the day. State: "Select a goal to work on today as readers. Record your goal in your reader's response notebook." Praise students for recording a goal. "Today, my goal is to follow four steps to monitor my use of the text coding strategy."

2. Agenda:

- Read text from book boxes

- Use the self-monitoring and TRACK procedures

- Share my thinking about the text

- Reflect on my goal

- Create a kidblog post to share my responses to text

3. Present text written at participant's instructional level. State: "Today, we will read . This story is about "

4. Ask questions to activate prior knowledge on the text topic; have participant use mind-mapping applications to create idea webs. State: "Can you think of ?" Make a web of ideas. Praise students for contributing ideas to the web.

5. Revisit the comprehension strategies for monitoring for meaning. State: "We will use the text coding strategy as we read. The word TRACK can help us remember to code the text and to monitor for meaning while we read." 
6. State the purpose of the comprehension strategies. State: "Remember that this strategy helps us read and comprehend complex literary and informational text (CCSS. ELA-Literacy.CCRA.R.10). When we code the text, we leave tracks of our thinking. We use this strategy to reflect on and react to text."

7. Display the TRACK mnemonic and sentence-starters related to the comprehension strategies. Say, "Last time we met, we used sentence-starters to talk about the strategy that we used while thinking aloud about the text." Hold up sentence-starters on sentence strips. "For the strategy ask questions, we started sentences with ..." Have students select the corresponding sentence strip. Provide praise for attempting/achieving the task. "For the strategy connect we used the sentence-starter..." Have students select the corresponding sentence strip. Provide praise for attempting/achieving the task. "When we monitored comprehension and realized that what we read did not make sense, we used...." Have students select the corresponding sentence strip. Provide praise for attempting/achieving the task. "What key word do we use to remind us how to TRACK our thinking?" Listen to responses, and accept TRACK. "Let's see if we can remember what each of the letters in the word TRACK stand for." Present reading comprehension strategy cue card. Cover up the card and reveal its contents letter by letter as students collaboratively recall the strategy. Provide praise for each attempt to recall the strategy.

Reading Strategy: Code the text to keep TRACK of your thinking.

Think about what I am reading.

React to the text.

Ask questions.

Connect.

Keep track of your thinking.

8. Present the self-monitoring checklist. State: "Today you will practice following four steps to help you use the TRACK strategy so you can monitor for meaning while you read. Let's look at the self-monitoring procedure card to learn what the four steps are.”

$\square$ I read the paragraph.

$\square$ I used the prompt card to recall the strategy steps.

$\square$ I applied the strategy to monitor for meaning.

$\square$ I coded the text to leave tracks of my thinking.

9. Explain the purpose of the self-monitoring checklist. State: "This card will help you use the TRACK strategy. You will use the card to make sure that you completed all of the steps involved in coding the text. Coding the text is a strategy that good readers use to comprehend what you read. I will show you how I use the card to monitor how I use the text coding strategy." 
10. Have participant read aloud from instructional-level text. Provide prompt, if needed, to check off this step on the checklist. Say, "Open the book and read aloud the first paragraph." Monitor to see whether participant checks off the first step on the checklists. If not, provide a prompt saying, "Check off the first step on the self-monitoring procedure checklist." As the participant to read aloud step 2 on the selfmonitoring procedure card.

11. Refer to the TRACK strategy card and think aloud about the first paragraph, saying, "T means [have participant supply response] and $\mathrm{R}$ means

[have participant supply response]. That's good! We are using the TRACK card to recall the steps of the strategy." Together check off the second step on the checklist.

12. Ask participant to think aloud about what he or she read in the first paragraph. Provide a model if no response is given. Say, "I am curious about ,' [This models using the letter A in track, step 3 on the checklist.] "I am going to code the text using because ." [This models step 4 on the checklist.]

13. Ask participant to continue reading from the text to a designated stopping point.

14. Ask the participant to think aloud about what he or she read. Provide a model if no response is given. Say, "A stands for ask questions. What does the author mean about ?" I think I am going to code the text using "??" because this is making me wonder.

15. Ask the participant to continue reading a designated stopping point.

16. Ask participant to think aloud about what he or she read. Provide a model if no response is given. Say, "C stands for connect. This reminds me of I am going to code the text using because "’

17. Say, "We used each letter to think about the text. So, I let's check off step three. Also, we coded the text to leave tracks of my thinking, so let's check off step four. Now, let's keep reading."

18. Ask the participant to state the purpose of the self-monitoring procedure. Provide a cue if needed, saying, "The four steps on the self-monitoring procedure card will help you use the TRACK strategy so you can monitor for meaning while you read."

19. Have participant select and read an instructional-level text. Each student in the group will read a different text. Provide students with sticky notes to code their thinking. Provide visual cues to show how to code the text. Rotate through the group and have students read portions of the text aloud. Note the level of word-reading accuracy 
demonstrated by participants (number of words read correctly divided by the total number of words read aloud). Record the date, the title of the text, the genre of the text, and other relevant information about the lesson on the formative assessment data recording form. Say, "For the next 10 minutes each of you will read from a book in your book box. As you read, use sticky notes to code the text. You can use the symbols ?? !! and * to leave tracks of your thinking. Begin reading silently. I will ask some of you to read aloud to me. Any questions?" [Answer questions.] "Begin."

20. Pose scripted literal and inferential comprehension questions. Paraphrase participants' responses. Record (+) for on-target responses and (-) for off-target responses on data sheets, along with date, title of text, and genre of text.

21. Ask participant to talk about the text as well as to explain how he or she used the targeted comprehension strategy. Use scripted prompts, including:

- What problems did you have while reading?

- What did you do to solve problems you had while reading?

- How do you know when you understand text?

22. After listening to responses and observing reading behavior, choose the performance level that best reflects the student's performance (i.e., assign a rubric score.) Record rubric score and targeted strategy on data sheet.

23. Ask participant to reflect on his or her goal. Say: "Look at the goal you recorded at the beginning of the session. Think about the progress you made toward meeting your goal. Make a note to show whether you met your goal."

24. Have participant verbally pose questions, make comments, and offer insights by posting to a weblog or by writing in a reader's response notebook. 
Lesson 6: Independent Practice Sessions

Materials:

- Reader's response notebooks

- Sentence-starters (This reminds me of, I am confused about)

- TRACK mnemonic

- Self-monitoring procedure card

- Sticky notes for coding the text

- Selection of instructional-level texts for each participant

- Scripted comprehension check questions to accompany each text

- Comprehension thinking strategy rubric

- Data recording forms

Note on Text Colors

Black $=$ Step by step instructions;

Blue $=$ Teacher's script;

Red $=$ Important reminders.

Purpose: Provide an opportunity for participants to practice using the self-monitoring procedure while reading and coding instructional-level text.

1. The lesson will begin by setting goals for the day. State: "Select a goal to work on today as readers. Record your goal in your reader's response notebook." Praise students for recording a goal.

2. Agenda:

- Read text from book boxes

- Use the self-monitoring and TRACK procedures

- Share my thinking about the text

- Reflect on my goal

- Create a kidblog post to share my responses to text

3. Present text written at participant's instructional level. State: "Today, we will read books from our book boxes."

4. Ask questions to activate prior knowledge on the text topic; have participant use mind-mapping applications to create idea webs. State: "Preview the text you select and create a web of ideas to activate your background knowledge."

5. Ask participant to state the purpose of the comprehension strategy. Say, "Be sure to use the text coding strategy as you read. How does this strategy help us when we read?" 
Listen to responses. Provide a cue, if needed: "The word TRACK can help us remember to code the text and to monitor for meaning while we read."

6. Display sentence-starters related to the comprehension strategies. Say, "While you read you can use our sentence-starters to think about the text." Hold up sentence-starters on sentence strips.

7. Display the TRACK mnemonic. Say, "What key word do we use to remind us how to TRACK our thinking?" Listen to responses, and accept TRACK. "What does each letters in the word TRACK stand for?" Present reading comprehension strategy cue card. Cover up the card and reveal its contents letter by letter as students collaboratively recall the strategy. Provide praise for each attempt to recall the strategy.

8. Present the self-monitoring checklist. State: "Today you will follow the four steps on the self-monitoring procedure card as you use the TRACK strategy. Let's look at the self-monitoring procedure card to learn what the four steps are."

$\square$ I read the paragraph.

$\square$ I used the prompt card to recall the strategy steps.

$\square$ I applied the strategy to monitor for meaning.

I coded the text to leave tracks of my thinking.

9. Provide participant with sticky notes. Direct participant to determine how and why to code the text and to leave tracks of thinking. Say, "Use the card to make sure that you complete all of the steps involved in coding the text." Pass out sticky notes. Ask students how and why to code the text. Provide a cue if needed, saying, "The four steps on the self-monitoring procedure card will help you use the TRACK strategy so you can monitor for meaning while you read."

10. Have participant select and read an instructional-level text. Each student in the group will read a different text. Provide students with sticky notes to code their thinking. Provide visual cues to show how to code the text. Rotate through the group and have students read portions of the text aloud. Note the level of word-reading accuracy demonstrated by participants (number of words read correctly divided by the total number of words read aloud). Record the date, the title of the text, the genre of the text, and other relevant information about the lesson on the formative assessment data recording form. Say, "For the next 10 minutes each of you will read from a book in your book box. As you read, use sticky notes to code the text. You can use the symbols ?? !! and * to leave tracks of your thinking. Begin reading silently. I will ask some of you to read aloud to me. Any questions?" [Answer questions.] "Begin." 
11. Pose scripted literal and inferential comprehension questions. Paraphrase participants' responses. Record (+) for on-target responses and (-) for off-target responses on data sheets, along with date, title of text, and genre of text.

12. Ask participant to talk about the text as well as to explain how he or she used the targeted comprehension strategy. Use scripted prompts, including:

- What problems did you have while reading?

- What did you do to solve problems you had while reading?

- How do you know when you understand text?

13. After listening to responses and observing reading behavior, choose the performance level that best reflects the student's performance (i.e., assign a rubric score.) Record rubric score and targeted strategy on data sheet.

14. Ask participant to reflect on his or her goal. Say: "Look at the goal you recorded at the beginning of the session. Think about the progress you made toward meeting your goal. Make a note to show whether you met your goal."

15. Have participant verbally pose questions, make comments, and offer insights by posting to a weblog or by writing in a reader's response notebook.

16. After participants complete 3 independent practice lessons, have them video record and narrate use of the comprehension strategy with the self-monitoring procedure. 


\section{APPENDIX M}

\section{LIST OF CULTURALLY RELEVANT TEXTS}

The following list represents texts written between 2.0 and 5.0 grade levels. Mentor Texts were read aloud to participants during modeling and guided practice sessions.

Mentor texts were deemed culturally relevant based on the similarities between participants' self-reported life experiences and the experiences of the characters in the fiction texts.

Nonfiction texts were aligned to topics that had been addressed through units of study in the science and social studies curriculum.

\section{Mentor Texts}

One Green Apple by Bunting (Fiction)

Four Feet Two Sandals by Williams (Fiction)

A Day's Work by Bunting (Fiction)

The Day of Ahmed's Secret by Lewin (Fiction)

My Name is Maria Isabel by Ada (Fiction)

Roberto Clemente: Pride of the Pittsburgh Pirates by Winter (Nonfiction)

A Drop of Water: A Book of Science and Wonder by Wick (Nonfiction)

One Well: The Story of Water on Earth by Strauss (Nonfiction)

Bread, Bread, Bread by Morris (Nonfiction) 
The following list represents texts written at levels K and L (2.0) and levels $\mathrm{M}$ and $\mathrm{N}$ (3.0).

Instructional-level texts were deemed culturally relevant if the following criteria were met: characters in the story represented the same age, gender, language, and culture of the participant; the setting was familiar to the participant; and the events in the story aligned with experiences the participant had shared.

Nonfiction texts addressed topics that had been presented through the science and social studies curriculum.

$\underline{\text { Instructional-Level Texts }}$

Sitti's Secrets by Nye

I Love Saturdays y domingos by Ada

In My Family: En mi familia by Garza

The Name Jar by Choi

Chato's Kitchen by Soto

Icy Watermelon by Galindo

Pepita Talks Twice by Lachtman

Jalapeno Bagels by Wing and Casilla

The Butterman by Alalou

Too Many Tamales by Soto

A Chair for My Mother by Williams

Amira's Totally Chocolate World by Mair

Owen by Henkes

Shark Swimathon by Murphy

Fireflies by Brinkloe

From Wheat to Bread by Taus-Bolstad

Wiggling Worms at Work by Pfeffer

National Geographic Readers: Storms by Goin

Zipping, Zapping, Zooming Bats by Earle 
Bug Out! The World's Creepiest Crawliest Critters by Clarke Hottest, Coldest, Deepest, by Jenkins

How Much Is A Million by Schwartz

What do you do with a Tail Like This? By Jenkins

Who Eats What by Lauber

Thinking About Ants by Brenner

Uncle Nacho's Hat by Rohmer

Kip: A Sea Otter by Taylor

Koko's Kitten by Patterson and Cohn

Super Storms by Simon

Watch Out! By Clarke

Tornado Alert by Branley

National Geographic Readers: Rocks and Minerals by Zoehfeld

Inside an Ant Colony by Fowler

Animals that Sting by Saxby

Amazing Grace by Hoffman

Amelia's Road by Altman

Everybody Bakes Bread by Dooley

An Eathworm's Life by Himmelman

Boom by Gutner 